\title{
Pattern selection in the visual cortex
}

\author{
Dissertation
}

\author{
zur Erlangung des Doktorgrades \\ der Mathematisch-Naturwissenschaftlichen Fakultäten \\ der Georg-August-Universität zu Göttingen
}

vorgelegt von

Matthias Kaschube

aus Bergisch Gladbach

Göttingen 2005 
Referent:

Korerefentin:

Tag der mündlichen Prüfung:
Prof. Dr. Theo Geisel

Prof. Dr. Annette Zippelius 22.12.2005 


\section{Contents}

\begin{tabular}{lll}
\hline 1 & Introduction & 7
\end{tabular}

2 Basics 13

2.1 Orientation maps in the mammalian visual cortex . . . . . . . . . . . . 13

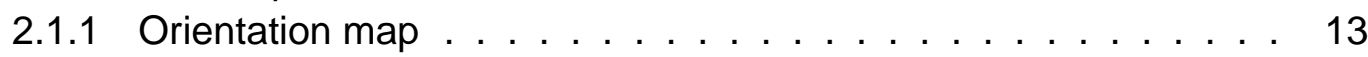

2.1 .2 Intra-cortical connectivity . . . . . . . . . . . . . . . . . . . 15

2.1 .3 Pinwheels . . . . . . . . . . . . . . . . . . . . . . . 15

2.1 .4 Hypercolumn and pinwheel density . . . . . . . . . . . . 16

2.1 .5 Development and the role of visual input . . . . . . . . . . . . . 17

2.2 The self-organizing orientation map . . . . . . . . . . . . . . . . . . . 19

2.2 .1 A pattern formation model . . . . . . . . . . . . . . . . . . . 19

2.2 .2 Weakly nonlinear analysis . . . . . . . . . . . . . . 21

2.2 .3 Pinwheel density . . . . . . . . . . . . . . . . . 23

3 Interareal coordination of column development in the visual cortex 25

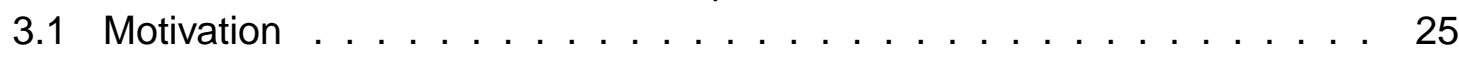

3.2 Methods . . . . . . . . . . . . . . . . . . . . . . . . . 25

3.2 .1 Animals . . . . . . . . . . . . . . . . . . 25

3.2 .2 Image processing . . . . . . . . . . . . . . . . . . . 26

3.2 .3 Spacing analysis . . . . . . . . . . . . . . . . . . . 26

3.2 .4 Accuracy and measurement errors . . . . . . . . . . . . . . . . 28

3.2 .5 Decomposition of variance $\ldots \ldots \ldots . . \ldots . . \ldots 28$

3.2 .6 Morphing . . . . . . . . . . . . . . . . . . . . . . . . . . . . . . . 29

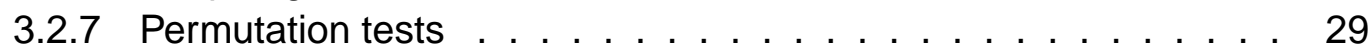

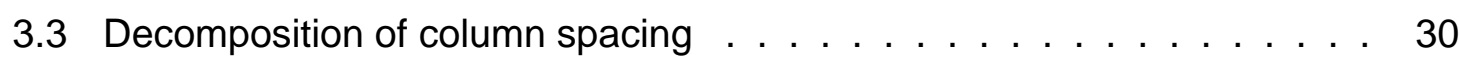

3.4 Mean column spacing . . . . . . . . . . . . . . . . . . . . . . . . . . . . 30

3.5 Systematic topographic variation . . . . . . . . . . . . . . . . . . . . . 30

3.6 Individual topographic organization . . . . . . . . . . . . . . . . . 33

3.7 Consolidation of column size matching . . . . . . . . . . . . . . . . . 35

3.8 Conclusion $\ldots \ldots \ldots \ldots \ldots \ldots \ldots$

4 Pinwheel density selection in the visual cortex 39

4.1 Introduction . . . . . . . . . . . . . . . . . . . . . . 39

4.2 Methods $\ldots \ldots \ldots \ldots$. . . . . . . . . . . . . . . . . . . . . . . . . . . . 39 


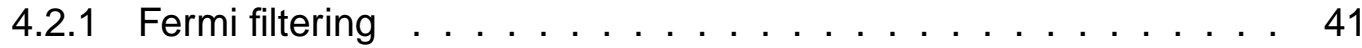

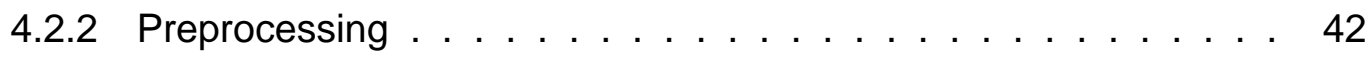

4.2 .3 Column spacing $\ldots \ldots \ldots \ldots$

4.2 .4 Pinwheel density estimation $\ldots \ldots \ldots \ldots$

4.2 .5 Pinwheel locations . . . . . . . . . . . . . . . . . . 44

4.2 .6 Nearest neighbor distances . . . . . . . . . . . . . . . . . 44

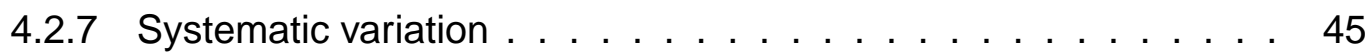

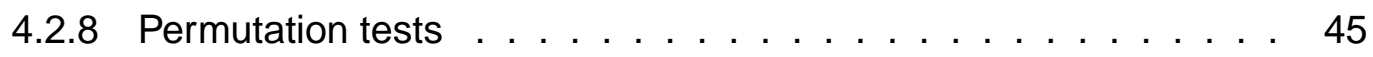

4.2 .9 Comparison of fully and semi automatic method. . . . . . . . . 45

4.2 .10 Measurement of column spacing by Fourier analysis . . . . . . 46

4.2 .11 Age-dependence . . . . . . . . . . . . . . . . 47

4.2 .12 Dependence of pinwheel density on high-pass filtering . . . . . 47

4.3 Universal pinwheel density in galago, ferret and tree shrew . . . . . . . 48

4.3 .1 Uncertainty of average pinwheel density estimation . . . . . . . 49

4.3 .2 Random maps . . . . . . . . . . . . . . . . . 50

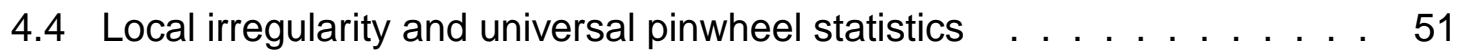

4.4 .1 Large scale selection . . . . . . . . . . . . . . . . 51

4.4 .2 Systematic inhomogeneity . . . . . . . . . . . . . . 52

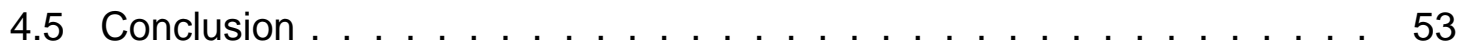

5 Theory of pinwheel density selection 55

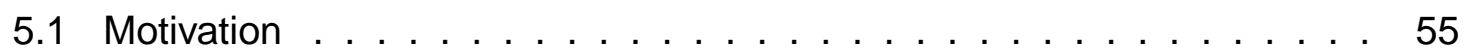

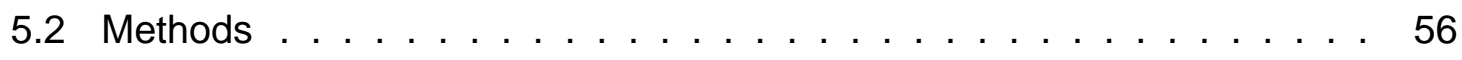

5.2 .1 The model . . . . . . . . . . . . . . . . . . 56

5.2 .2 Numerical integration . . . . . . . . . . . . . . . . 56

5.2 .3 Linear wavelength selection. . . . . . . . . . . . . . . . . . . 59

5.2 .4 Pinwheel identification $\ldots \ldots \ldots$. . . . . . . . . . . . . . 61

5.3 Pinwheel densities near criticality . . . . . . . . . . . . . . 62

5.3 .1 Pinwheel densities for intermediate interaction range . . . . . . 62

5.3 .2 Limit of large interaction range: Planform anisotropy . . . . . . 63

5.3 .3 Distribution of planform anisotropies . . . . . . . . . . . 69

5.3 .4 Pinwheel density in the large $n$ limit . . . . . . . . . . . 70

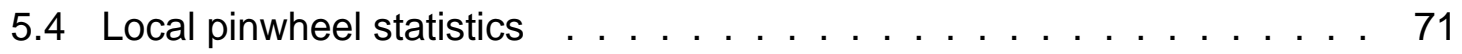

5.4 .1 Count statistics $\ldots \ldots \ldots \ldots \ldots \ldots$

5.4 .2 Nearest neighbor statistics . . . . . . . . . . . . . . . 72

5.5 Pinwheel densities away from criticality $\ldots \ldots \ldots \ldots \ldots$

5.5 .1 Transient states $\ldots \ldots \ldots \ldots \ldots \ldots \ldots \ldots$

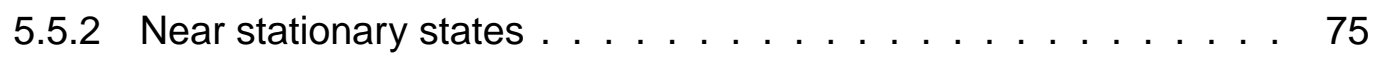

5.5 .3 Robust selection . . . . . . . . . . . . . . . . . . . . 76

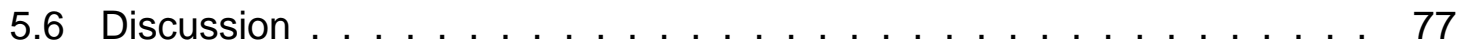


6 Predicted pinwheel kinetics during development 79

6.1 Motivation . . . . . . . . . . . . . . . . . . . . 79

6.2 Methods . . . . . . . . . . . . . . . . . . . . . . . 80

6.2 .1 Pinwheel tracking . . . . . . . . . . . . . . . . . . 80

6.2 .2 Boundary conditions . . . . . . . . . . . . . . . . . 80

6.3 Development of orientation maps . . . . . . . . . . . . . . . . . . . . 81

6.4 Development of pinwheel statistics . . . . . . . . . . . . . . . . . . . 84

6.4 .1 Pinwheel density . . . . . . . . . . . . . . . . 85

6.4 .2 Nearest neighbor statistics . . . . . . . . . . . . . . . . . 86

6.4 .3 Annihilation and creation . . . . . . . . . . . . . . . . . . . 87

6.4 .4 Movement and speed . . . . . . . . . . . . . . . . . . 90 90

6.5 Temporal scaling of map dynamics . . . . . . . . . . . . . . . . . . . . 92

6.6 Pinwheel densities in a bounded area . . . . . . . . . . . . . . . . . . . 99

6.7 Discussion . . . . . . . . . . . . . . . . . . . . . . . . . 102

7 Soft spots in the functional architecture of

7.1 Introduction . . . . . . . . . . . . . . . . . . . . 105

7.2 Modeling intracortical microstimulation (ICMS) . . . . . . . . . . . 106

7.3 Methods . . . . . . . . . . . . . . . . . . . . 108

7.3 .1 Numerical integration . . . . . . . . . . . . . . . . . . . 108

7.3 .2 Pinwheel analysis . . . . . . . . . . . . . . . . . . . . . . . . . . . . . . . . . . . . . . . . . .

7.4 A numerical ICMS experiment . . . . . . . . . . . . . . . . . . . . 109

7.4 .1 Transient reorganization . . . . . . . . . . . . . . . . . . . 109

7.4 .2 Persistent reorganization . . . . . . . . . . . . . . . . . . . . . . . . . . . . . . . . . . . . . . . .

7.4 .3 Spatial-temporal dynamics . . . . . . . . . . . . . . . . . . . . 111

7.5 Soft spots in the orientation map . . . . . . . . . . . . . . . . . . 112

7.5 .1 Dependence of reorganization on ICMS site . . . . . . . . . 112

7.5 .2 Induced pinwheel dynamics . . . . . . . . . . . . . . . 113

7.5 .3 Independence from local map properties . . . . . . . . . . . . . 114

7.5 .4 Stimulation strength and duration . . . . . . . . . . . . . . . . . 115

7.5 .5 Amplitude equations . . . . . . . . . . . . . . . . . . 116

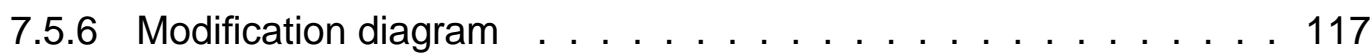

7.6 Soft spots implied by map layout $\ldots$. . . . . . . . . . . . . . . . . . . . . 120

7.7 Predicting soft spots . . . . . . . . . . . . . . . . . . . . . . . . . . . . . . . . . . . . . . . . . . . . . . . . . .

7.7 .1 Softness . . . . . . . . . . . . . . . . . . . 122

7.7 .2 Predictability . . . . . . . . . . . . . . . . . . . 123

7.7 .3 Predicting the full dynamics . . . . . . . . . . . . . . . . . . . 127

7.8 Discussion . . . . . . . . . . . . . . . . . . . . . . . . . . . . . . . . . . . 130 
8 Reorganization of visual cortical orientation maps by intracortical microstimulation

8.1 Introduction . . . . . . . . . . . . . . . . . . . . . . 133

8.2 Experimental paradigm and analysis methods . . . . . . . . . . . . . . 134

$8.2 .1 \quad$ Alignment of maps . . . . . . . . . . . . . . . . . . . . . . . 134

8.2 .2 Preprocessing . . . . . . . . . . . . . . . . . . . . . . . . . . . . 136

8.2 .3 Power spectrum . . . . . . . . . . . . . . . . . . . . . . . . . . 136

8.2 .4 Local similarity . . . . . . . . . . . . . . . . . . . . . . . . . . . . . 136

8.2 .5 Pinwheel analysis . . . . . . . . . . . . . . . . . . . . . . . 136

8.2 .6 Softness . . . . . . . . . . . . . . . . . . . . . . . 137

8.3 Persistent and transient reorganization . . . . . . . . . . . . . . . . 137

8.3 .1 Persistent reorganization . . . . . . . . . . . . . . . . . . . 138

8.3 .2 Transient reorganization. . . . . . . . . . . . . . . . . . . . . . 138

8.3 .3 Two classes of experiments . . . . . . . . . . . . . . . . . . . . . . . 139

8.4 Spatial-temporal dynamics . . . . . . . . . . . . . . . . . . . . . . . . 141

8.5 Induced pinwheel dynamics . . . . . . . . . . . . . . . . . . . . . . . 142

8.5 .1 Pinwheel movement . . . . . . . . . . . . . . . . . . . . . . . . 143

8.5 .2 Pinwheel density . . . . . . . . . . . . . . . . . . . . . 143

8.6 Soft spots in the visual cortex . . . . . . . . . . . . . . . . . . . . 144

8.6 .1 Softness . . . . . . . . . . . . . . . . . . . . . 144

8.6 .2 Predicting soft spots in the visual cortex . . . . . . . . . . . . . . 145

8.7 Discussion . . . . . . . . . . . . . . . . . . . . 146

$\begin{array}{lll}9 & \text { Conclusion } & 149\end{array}$

Bibliography 153

\begin{tabular}{ll}
\hline Bibliography & 153
\end{tabular}

\begin{tabular}{ll}
\hline Acknowledgments & 163
\end{tabular} 


\section{Introduction}

Measure what is measurable, and make measurable what is not so.

(Galileo Galilei)

In many systems, regular spatially extended structures can arise from interactions between its component parts [3] 23]. For instance, in vertically shaken, shallow granular beds, a rich variety of patterns including stripes, squares and hexagons form spontaneously [58, 60] in a way that can be explained by the inelastic collision of grains together with a randomization of their horizontal velocities by shaking [84]. Pattern formation is observed in fluids [8], chemical reactions, epidemics and morphogenesis [37, 47, 96]. One hallmark of pattern formation is the selection of patterns within a particular class (e.g. stripes or hexagons). The class primarily depends on the form of the interactions of the constituent parts and the process of formation is frequently robust with respect to the details of the system and against noise, explaining why patterns belonging to the same class can be observed in different systems often composed of very different materials and operating through different microscopic interactions [3, 23].

Evidence for pattern selection in the brain comes from experiments inducing substantial manipulations of neural activity. For instance, implanting tadpoles a third eye leads to a segregation of alternating eye specific domains with characteristic length scale in the normally monocularly innervated tectum (Fig. 1.1] [19]. In cat visual cortex, changing the relative strength of synaptic inhibition and excitation was shown to change the spacing of columns that are dominated by one eye or the other [38]. Viewed from a dynamical systems perspective, these and other examples of activity-dependent remodeling of neuronal circuitry resemble processes of dynamical pattern formation.

In this thesis, we explore for the example of the orientation map in the visual cortex in how far a pattern formation framework can capture the phenomenology of large-scale structure formation in the brain. We hope that an appropriate and transparent description of these processes may help to identify the basic principles underlying the development and modifiability of neural networks.

In the visual cortex, as in most areas of the cerebral cortex information is processed in a 2-dimensional (2D) array of functional modules, called cortical columns [22, 50, 63]. Individual columns are groups of neurons extending vertically through- 

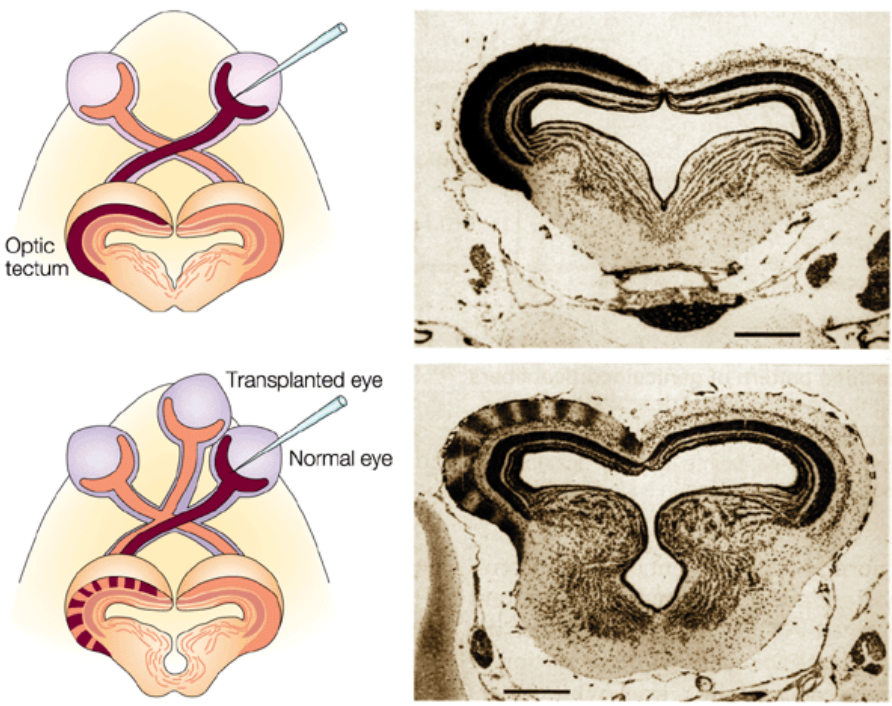

Nature Reviews | Neuroscience
Figure 1.1: Pattern selection in the brain by activitydependent competition. In a normal frog, retinal ganglion cells from each eye project to the contralateral tectum. Implanting tadpoles a third eye leads to a segregation of alternating eye specific domains with a characteristic length scale. From [46].

out the entire cortical thickness that share many functional properties. Orientation columns in the visual cortex are composed of neurons preferentially responding to visual contours of a particular stimulus orientation [40]. In a plane parallel to the cortical surface, neuronal selectivities vary systematically, so that columns of similar functional properties form organized 2D patterns, known as functional cortical maps. In the case of orientation columns, this $2 \mathrm{D}$ organization is named the orientation map. An orientation map in the left brain hemisphere covers the complete cortical representation of the right visual hemifield and vice versa. Whereas the preferred visual field position of neurons varies roughly gradually with location in the orientation map, their preferred orientation varies repetitively with a characteristic length scale in the millimeter range termed column spacing. The squared column spacing, in turn, defines the fundamental unit of surface area in an orientation map often called a hypercolumn.

The orientation map is characterized by so called pinwheels [14], regions in which columns preferring all possible orientations are organized around a common center in a radial fashion [9, 10, 93]. At the center of pinwheels, the normally smooth orientation map is discontinuous and the preferred orientation of nearby columns changes by 90 degrees. Pinwheels are abundant in the visual cortex of higher mammals such as primates and carnivore [99]. They occur numerously in the primary visual area V1, but also in visual areas V2, V3 [109] and MT [11] with typically a few pinwheels per hypercolumn. Thus, even a point-like stimulus like a star in the sky evokes activity in a cortical region large enough to contain typically a few pinwheels. Despite their abundance, the role of pinwheels remains 
enigmatic. It was reported that in the vicinity of pinwheel centers neurons exhibit normal orientation selectivity [4] [56] (but see also [70]). Although plausible, it remains controversial whether the organization of pinwheels influences visual processing [26, 80].

What is guiding the development of the orientation map? Presently, biological researchers favor two alternative hypotheses, in the tradition of the classical nurture vs. nature dichotomy in systems neuroscience. The first hypothesis states that the formation of orientation columns is a dynamical process dependent on neuronal activity and sensitive to visual experience [88, 92]. This is suggested not only by the time line of normal development, but also receives support from various experiments manipulating the sensory input to the cortex. For instance, in the ferret, re-routing projections from the retina to the auditory pathway induces the formation of orientation selective neurons arranged in a pinwheel like pattern in the primary auditory cortex [83]. Moreover, the comparison of development under conditions of modified visual experience demonstrates that adequate visual experience is essential for the complete maturation of orientation columns and that impaired visual experience, as with experimentally closed eye-lids can suppress or impair the formation of orientation columns [21, 103]. This observation suggests that the capability to form a system of orientation columns is intrinsic to the learning dynamics of the cerebral cortex given appropriate inputs. Moreover, the comparison of development under conditions of modified visual experience demonstrates that adequate visual experience is essential for the complete maturation of orientation columns and that impaired visual experience, as with experimentally closed eyelids can suppress or impair the formation of orientation columns [21, 103]. This is consistent with the hypothesis that sensory input instructs cortical circuitry [20].

However, a number of experiments suggest that the basic layout of orientation maps is only barely susceptible to activity-dependent modification. In animals which have been reared in complete darkness, seemingly normal orientation maps form at about the same time than in normal animals [103] and a restructuring of the orientation map by manipulating sensory experience has never been reported. Rearing kittens in a striped environment consisting of a single orientation caused up to twice as much cortical surface area to be devoted to the experienced orientation, but neither eliminate the representation of the orthogonal orientation that was never experienced by the animals completely nor changed the typical layout of the orientation map with its typical periodicity and its abundant pinwheels [82]. Moreover, in kittens with alternating monocular experience from birth, achieved by sequential monocular deprivation, similar orientation maps were found for the left and right eye [36].

This apparent stability of the orientation map has revived the second hypothesis which has been proposed originally by Hubel and Wiesel, namely that orientation maps are prespecified in detail by genetic factors [41] (but see also [31, 106] for alternative explanations). Indeed, a recent quantitative analysis by the author provided 
evidence for a genetic control on properties of the orientation map such as the spacing and form of columns [44] and on the system of ocular dominance columns [45]. In this view, sensory experience plays only a permissive role, i.e. the presence of activity is sufficient for generating maps, but which map is selected does not depend on the patterns of neuronal activity. A combined scenario, supported by more recent work, favors an initial phase in which circuitry is established without much sensory instruction, followed by a period of refinement through detailed sensory input [25, 34, 46].

Following the first hypothesis, a number of mathematical models for the activity dependent development of orientation selectivity were proposed $30,32,61,62,65$, 67, 92, 100, 104]. These models show that orientation selective neurons can arise by activity driven self-organization of cortical circuitry. In this picture, spontaneous symmetry breaking in the developmental dynamics of the cortical network underlies the emergence of cortical selectivities such as orientation preference [61]. However, most of the work focused only on the initial phase of development, partially due to a lack of computer power at this time. It was first noted by Wolf and Geisel [107] that orientation maps with a realistic number of pinwheels are expected to be unstable in many proposed models.

More sophisticated approaches were proposed to overcome this problem. Solutions containing a large number of pinwheels were stabilized by assuming a common symmetry of orientation and visual field representation [49] 94]. An alternative approach emphasizing the cost of cortical wiring showed that there are pinwheel rich solutions minimizing the total length of wiring [18]. This model not necessarily meant to describe ontogenetic development of the orientation map, but rather its optimization under evolution. However, in both approaches, orientation maps are periodic, a property which is not shared by the orientation map in the visual cortex. A third approach argued that fixed clustered long-range intraareal connections may provide a scaffold for orientation maps stabilizing even realistic solutions [86]. However, a fixed scaffold questions the necessity of plasticity during development and seems rather unrealistic, since long-range connections also develop experience dependent.

A recent and highly promising approach stressing the analogy to pattern forming systems showed that pinwheels can be stabilized by activity dependent longrange interactions [105]. A phenomenological order parameter field model based on the Swift-Hohenberg equation [89] was proposed in which orientation maps arise from a supercritical bifurcation of Turing-type. The stabilizing nonlinearity includes only key features of visual cortical organization and is constraint by biologically plausible symmetry assumptions. Near the bifurcation point, the model exhibits quasiperiodic attractors resembling orientation maps in the visual cortex. The convergence towards an attractor of this developmental dynamics can be interpreted as an optimization process. The analogy to pattern formation appears plausible. Like other systems where pattern formation has been observed [3, 23], 
the orientation map arises probably from an initially unselective state, it exhibits a typical periodicity and a spatial extension at least an order of magnitude larger than the basic periodicity length. Adopting the framework provided by the theory of pattern formation, the model can be treated analytically near bifurcation. Moreover, by this approach, even large cortical regions can be modeled numerically which would be impossible in a microscopical model given that already a few hypercolumns require the description by $\sim 10^{6}$ synaptic strengths.

Thus, a variety of models have been proposed, but do they provide an appropriate description of the organization and development of the visual cortical orientation map? This question is not easy to answer despite the fact that a large number of experimental studies have been accumulated over the past years. The reason for this is primarily that quantitative predictions of the models were not tested experimentally. Recordings only provide a qualitative picture of orientation maps and the experimental data has barely been analyzed quantitatively mainly because of two reasons one of technical and one of more conceptual nature. Technically, orientation maps, like most data from the brain, are significantly contaminated with noise which renders a quantitative estimation at least difficult. Even more importantly, neural structures are often conceptualized as elaborately designed to fulfill the specific functional demands of a particular brain region for a distinct species. An accurate quantitative description aiming to identify rules or laws then may appear inappropriate or useless. We will provide evidence in this thesis that is hard to reconcile with this viewpoint. Progress in the field of neural map development, we believe, relies primarily on an precise quantitative characterization of experimental data.

In this thesis, we investigate pattern selection in the visual cortex. We quantitatively characterize the organization of pinwheels in the visual cortex and interprete the observed statistics by means of a phenomenological pattern formation model that reproduces many aspects quantitatively. We study induced pattern selection in both the model and in experimental data which leads us to the novel concept of soft spots, that are locations at which a neural network is highly modifiable by stimulation.

We begin with an investigation of column spacing selection in different visual cortical areas from the left and right brain hemisphere. We present a quantitative method for analyzing locally the column spacing in visual cortical orientation maps. A decomposition of local column spacing into the three parts average, systematic, and individual variation reveals a matching of column spacing in remote but connected brain regions, in individual animals and consolidating with age. Next, we calculate the density of pinwheels, i.e. the number of pinwheels per hypercolumn, in large data sets from three different species. Based on the local column spacing, we develop a novel method for the identification of pinwheels that is largely insensitive to noise. We find, that the average pinwheel density and the spatial variation of pinwheels, expressed by the count-statistics and the nearest 
neighbor statistics, are universal in the analyzed species but different in comparable random maps. The average pinwheel density is in all three species statistically indistinguishable from the mathematical constant $\pi$.

We analyze the organization of pinwheels in the abovementioned pattern formation model. We find that this model reproduces the observed pinwheel statistics in a large and apparently realistic parameter regime. For solutions close to the bifurcation point, we show analytically that in the limit of long-range interactions, the average pinwheel density converges to $\pi$. Numerically, we show that away from the bifurcation point and for a realistic interaction ranges, soon after maps arise from the initially unselective state, an average pinwheel density close to $\pi$ is robustly selected and remains largely preserved throughout development. Next, to characterize the organization of the orientation map during development, we investigate pinwheel annihilation and creation rates, survival probabilities, pinwheel path lengths and pinwheel speed. These quantities show, that the strength of reorganization decays as a power law during development, but with different exponents before and after a characteristic time. These quantities capture more sensitively the reorganization of the map during development providing guidelines for future experimental investigations.

In the remainder of this work we study induced reorganization in orientation maps. Extending the pattern formation model we include the impact of a localized stimulation. Numerical simulations show an induced pinwheel dynamics reminiscent to normal development. At particular locations, so-called soft spots, the stimulation results in a persistent and large-scale structural modification. An analysis based on the amplitude formalism indicates that these soft spots are largely a consequence of the complex structure of the orientation map. A quantity named softness predicts the location of potential soft spots from the layout of the map. The predictions of the model are compared to experiments using direct electrical intracortical microstimulation (ICMS) in visual cortical orientation maps. Assessing quantitatively the induced organization, we confirm the movement, annihilation and creation of pinwheels. The considerable variation of the observed modification is consistent with the interpretation of soft spots in the visual cortex. Preliminary results suggest that potential soft spots can be confined by the softness.

This thesis is organized as follows. The following Chapter 2 briefly describes biological basics and introduces the pattern formation model underlying all theoretical investigations. In Chapter 3, we present a method for quantifying local column spacing and identify its selection in different brain regions. The analysis of pinwheels and their organization in the visual cortex is shown in Chapter 4 . Pinwheels in the model are studied in stationary solutions in Chapter 5 and during development in Chapter 6. In Chapter 7wwe explore induced reorganization in the model and compare the predictions to experiment in Chapter 8 . The main results and consequences are provided in Chapter 9 at the end. 


\section{Basics}

If the mat was not straight, the master would not sit.

(Confucius)

In the following, we outline the state of research in this field and previous work this theses is based on. In parts, this chapter follows the presentation in [105]. In the first section, we describe the system of orientation columns in the visual cortex, its formation during development and its plasticity. In the second section, we introduce a pattern formation model for the development of orientation maps in the visual cortex.

\subsection{Orientation maps in the mammalian visual cortex}

\subsubsection{Orientation map}

In the visual cortex, as in most areas of the cerebral cortex, information is processed in a 2-dimensional (2D) array of functional modules, called cortical columns [22 [50]. Individual columns are groups of neurons extending vertically throughout the entire cortical thickness that share many functional properties. Orientation columns in the visual cortex are composed of neurons preferentially responding to visual contours of a particular stimulus orientation [39]. In a plane parallel to the cortical surface, neuronal selectivities vary systematically, so that columns of similar functional properties form highly organized 2D patterns, known as functional cortical maps (Fig. 2.1). In the case of orientation columns, this $2 \mathrm{D}$ organization is characterized by so called pinwheels, regions in which columns preferring all possible orientations are organized around a common center in a radial fashion [9]. 93.

Experimentally, the pattern of orientation preferences can be visualized using optical imaging methods [7 9]. Optical imaging of intrinsic signals is based on the fact that the optical properties differ in active vs. less active parts of the cortex [11]. This is utilized to record patterns of activity from light reflectance. In a typical experiment, the activity patterns $E_{k}(\mathbf{x})$ produced by stimulation with a grating of orientation $\theta_{k}$ are recorded. Here $\mathbf{x}$ represents the location of a column in the cortex. Using the activity patterns $E_{k}(\mathbf{x})$, a field of complex numbers $z(\mathbf{x})$ can be 


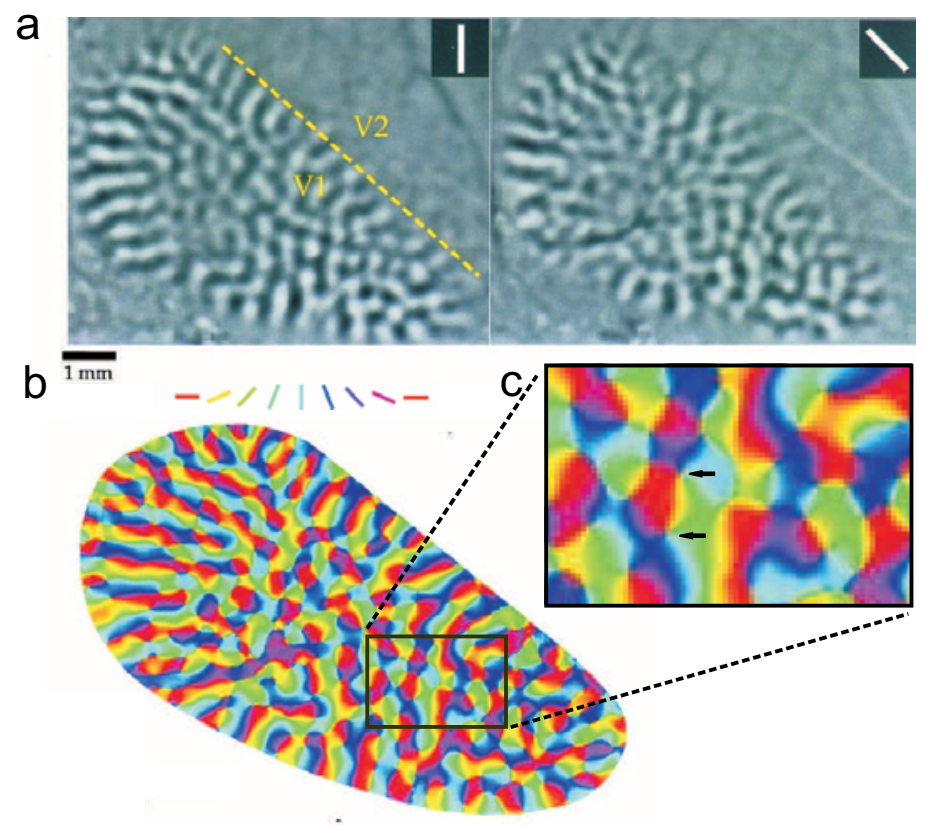

Figure 2.1: Patterns of orientation columns in the primary visual cortex of a tree shrew visualized using optical imaging of intrinsic signals (modified from [13). a, Activity patterns resulting from stimulation with vertically and obliquely oriented gratings. White bars depict the orientation of the visual stimulus. Activated columns are labeled dark grey. The used stimuli activate only columns in the primary visual area $\mathrm{V} 1$. The patterns thus end at the boundary between areas $\mathrm{V} 1$ and $\mathrm{V} 2$. $\mathbf{b}$, The pattern of orientation preferences calculated from such activity patterns. The orientation preferences of the columns are color coded as indicated by the bars. A part of the pattern of orientation preferences is shown at higher magnification in c. Two pinwheel centers of opposite topological charge are marked by arrows.

constructed that completely describes the pattern of orientation columns:

$$
z(\mathbf{x})=\sum_{k} e^{i 2 \theta_{k}} E_{k}(\mathbf{x}) .
$$

The pattern of orientation preferences $\vartheta(\mathbf{x})$ is then obtained from $z(\mathbf{x})$ as follows:

$$
\vartheta(\mathbf{x})=\frac{1}{2} \arg (z) \text {. }
$$

Typical examples of such activity patterns $E_{k}(\mathbf{x})$ and the patterns of orientation preferences derived from them are shown in Fig. 2.1. Numerous studies confirmed that the orientation preference of columns is an almost everywhere continuous function of their position in the cortex. Columns with similar orientation preferences occur next to each other in so called iso-orientation domains [91]. 
Typically, not the entire area V1 is accessible to optical imaging methods. Orientation columns in a complete visual area can be labeled by radioactive $2-\left[{ }^{14} \mathrm{C}\right]-$ deoxyglucose and visualized in flat-mount sections [51]. A disadvantage with this method is that the representation of only one stimulus orientation can be labeled.

\subsubsection{Intra-cortical connectivity}

Visual cortical neurons are embedded in densely connected networks [15]. Besides a strong connectivity vertical to the cortical sheet between neurons from different layers within a column, neurons also form extensive connections horizontal to the cortical surface linking different orientation columns. These connections extend for several millimeters parallel to the cortical surface and are therefore called long-range horizontal connections. As shown in Fig. 2.2 for the example of the tree shrew, these connections are clustered primarily connecting domains of similar orientation preference. They have been observed in various mammals [13, 54, 87, 103] and repeatedly hypothesized to play an important to role in visual processing tasks such as contour integration.

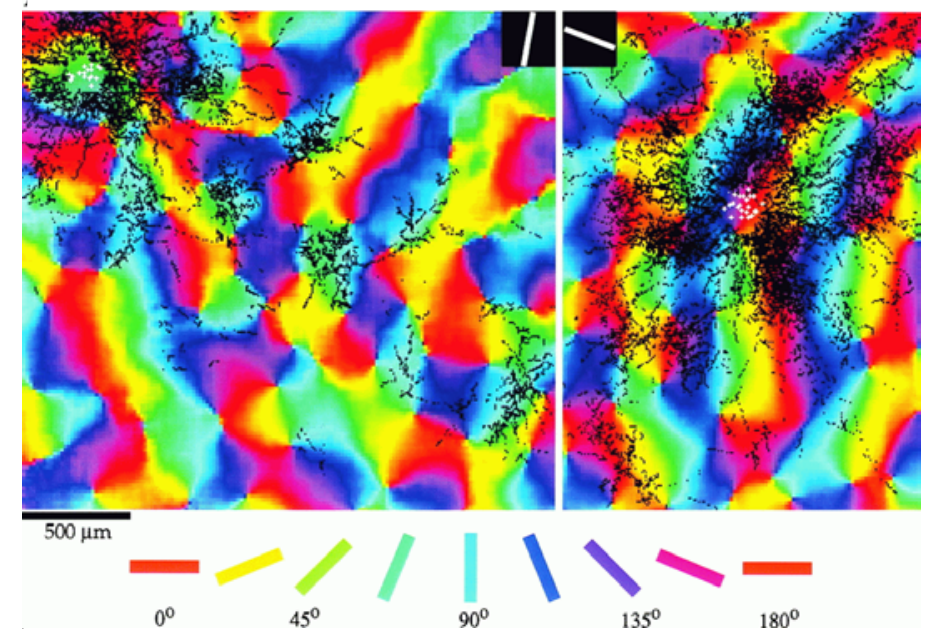

Figure 2.2: Long-range horizontal connections extend over several millimeters parallel to the cortical surface (tree shrew, superimposed on the orientation preference map). White symbols indicate locations of cells that were filled by a tracer (biocytin). Labeled axons are indicated by black symbols. From [13.

\subsubsection{Pinwheels}

It was found experimentally that at abundant locations the iso-orientation domains are arranged radially around a common center [9, 10]. Around these pinwheel [14] centers, stimulus orientations are represented in circular fashion (Fig. 2.1p, black frame). Such an arrangement had been previously hypothesized on the basis of electrophysiological experiments [1, 93] and theoretical considerations [90]. The regions exhibiting this kind of radial arrangement were termed pinwheels [14]. 
The centers of pinwheels are point discontinuities of the field $\vartheta(\mathbf{x})$ where the mean orientation preference of nearby columns changes by 90 degrees. They can be characterized by a topological charge $q$ which indicates in particular whether the orientation preference increases clockwise around the center of the pinwheel or counterclockwise,

$$
q_{i}=\frac{1}{2 \pi} \oint_{C_{j}} \nabla \vartheta(\mathbf{x}) d \mathbf{s},
$$

where $C_{j}$ is a closed curve around a single pinwheel center at $\mathbf{x}_{i}$. Since $\vartheta$ is a cyclic variable within the interval $[0, \pi]$ and up to isolated points is a continuous function of $\mathbf{x}, q_{i}$ can only have the values

$$
q_{i}=\frac{n}{2}
$$

where $n$ is an integer number [59]. If its absolute value $\left|q_{i}\right|$ is $1 / 2$, each orientation is represented exactly once in the vicinity of a pinwheel center. Pinwheel centers with a topological charge of $\pm 1 / 2$ are simple zeros of $z(\mathbf{x})$. In experiments only pinwheels that had the lowest possible topological charge $q_{i}= \pm 1 / 2$ are observed. This means there are only two types of pinwheels: those whose orientation preference increases clockwise and those whose orientation preference increases counterclockwise. This organization has been confirmed in a large number of species and is therefore believed to be a general feature of visual cortical orientation maps [4, $6,12,101]$.

If the orientation map changes smoothly over time, the entire topological charge $Q_{A}$ of a given area $A$ is invariant as long as no pinwheel transgresses the boundary of the area [59]. If the pattern contains only pinwheels with $q_{i}= \pm 1 / 2$, then only three qualitatively different modifications of the pinwheel configuration are possible. First, movement of the pinwheel within the area; second, generation of a pair of pinwheels with opposite topological charges; third, the annihilation of two pinwheels with opposite topological charge when they collide. Only these transformations conserve the value of $Q_{A}$ and are therefore permitted. A small variation of the pattern does not alter the number of pinwheels, a property called topological stability.

\subsubsection{Hypercolumn and pinwheel density}

Neighboring iso-orientation domains preferring the same stimulus orientation exhibit a typical lateral spacing $\Lambda$ in the range of $1 \mathrm{~mm}$, rendering the pattern of preferred orientations roughly repetitive $[39,[50]$. The column spacing $\wedge$ determines the size of the cortical hypercolumn, which is considered to be the basic processing unit of the visual cortex [22, 44]. The hypercolumn is loosely defined as the cortical region representing all stimulus features (e.g. orientation, spatial frequency, ocular dominance) at a given visual field position. We define the size of a hyper 
column by $\Lambda^{2}$. The pinwheel density is defined as the number of pinwheels per unit area $\Lambda^{2}$. By this definition, the pinwheel density is independent of the spacing of columns and dimension-less.

\subsubsection{Development and the role of visual input}

In normal development, orientation columns first form at about the time of eye opening [17, 21, 103] which for the ferret is approximately at post natal day (PD) 31. As shown in Fig. 2.3 by the time line of early cortical development for the ferret [88], this is just a few days after neurons first respond to visual stimuli. A subset of neurons show orientation preference from that time on, but the adult pattern is not attained until seven weeks after birth [16]. Roughly clustered horizontal connections are present by around PD 27 [75].

What is guiding the development of the orientation map? Presently, there exist two mutually excluding hypotheses. The first hypothesis states that the formation of orientation columns is a dynamical process dependent on neuronal activity and sensitive to visual experience [88, 92]. This is suggested not only by the time line of normal development, but also receives support from various experiments manipulating the sensory input to the cortex. Most intriguingly, when visual inputs are rewired to drive what would normally become primary auditory cortex, orientation selective neurons and a pattern of orientation columns even forms in this brain region that would normally not at all be involved in the processing of visual information [83, 88]. This observation suggests that the capability to form a system of orientation columns is intrinsic to the learning dynamics of the cerebral cortex given appropriate inputs. Moreover, the comparison of development under conditions of modified visual experience demonstrates that adequate visual experience is essential for the complete maturation of orientation columns and that impaired visual experience, as with experimentally closed eye-lids can suppress or impair the formation of orientation columns [21, 103]. This is consistent with the hypothesis that sensory input instructs [20] cortical circuitry. However, a number of experiments suggest that the basic layout of orientation maps is only barely susceptible to activity-dependent modification. In animals which have been reared in darkness, seemingly normal orientation maps form at about the same time than in normal animals 103 and a restructuring of the orientation map by manipulation of sensory experience has never been reported. Rearing kittens in a striped environment consisting of a single orientation caused up to twice as much cortical surface area to be devoted to the experienced orientation, but neither eliminate the representation of the orthogonal orientation completely nor changed the typical layout of the orientation map with its typical periodicity and its abundant pinwheels [82]. Moreover, in kittens with alternating monocular experience from birth, achieved by sequential monocular deprivation, similar orientation maps were found for the 


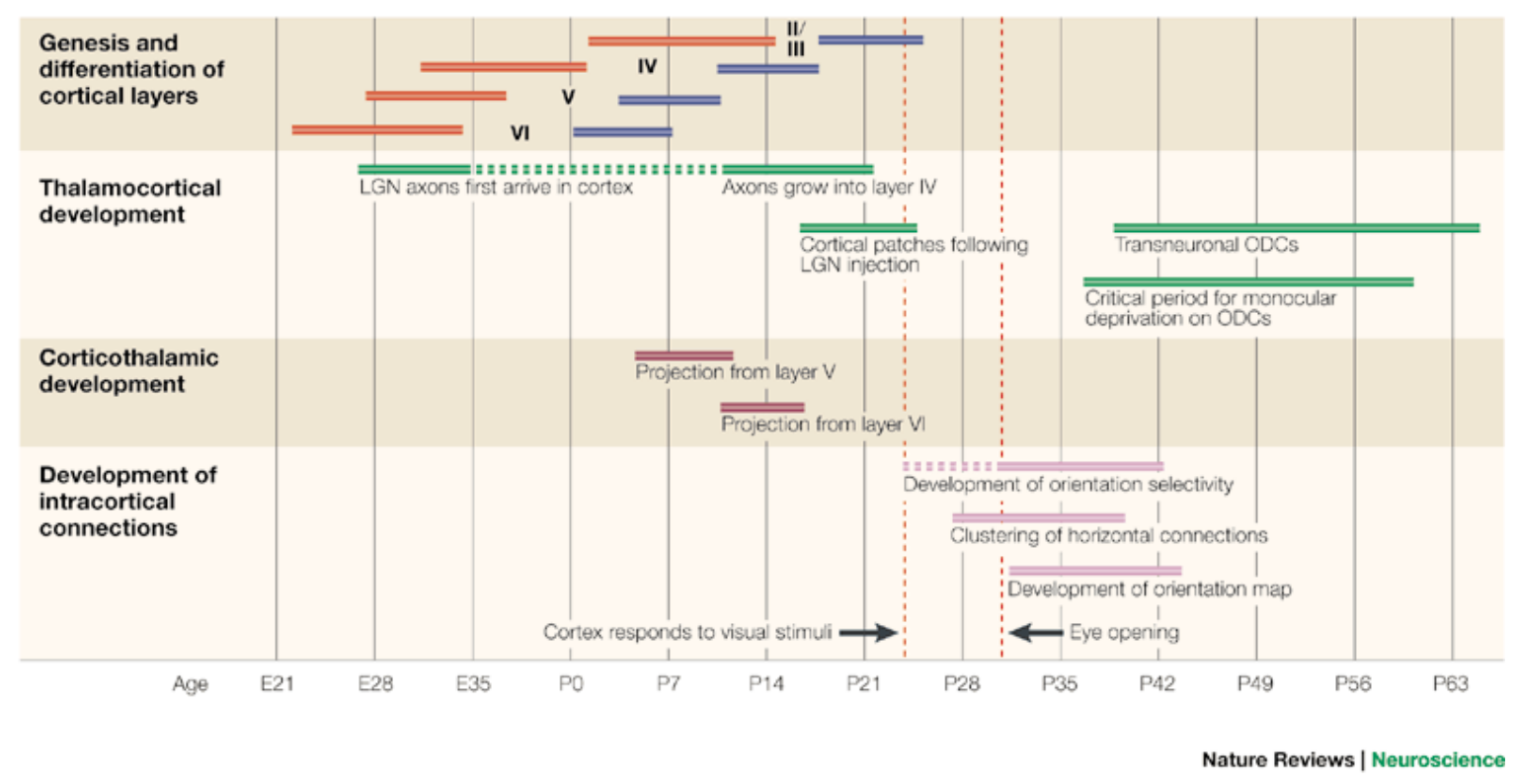

Figure 2.3: Time line illustrating many of the events during the normal development of the visual cortex. Orientation maps form at the time of eye opening a few days after the onset of responsiveness in the visual cortex. From [88].

left and right eye [36].

This apparent stability of the orientation map has revived the second hypothesis which has been proposed originally by Hubel and Wiesel, namely that orientation maps are prespecified in detail by genetic factors [41] (but see also [31, 106] for alternative explanations). Indeed, a recent quantitative analysis by the author provided evidence for a genetic control on properties of the orientation map such as the spacing and form of columns [44] and on the system of ocular dominance columns [45]. In this view, sensory experience plays only a permissive role, i.e. the presence of activity is sufficient for generating maps, but which map is selected does not depend on the patterns of cortical activity. A combined scenario, supported by more recent work, favors an initial phase in which circuitry is established without much sensory instruction, followed by a period of refinement through detailed sensory input [25, 46].

To approach this puzzle about the potential reorganizational capabilities of the visual cortex, Godde et al. [35] altered the intracortical activity pattern, instead of manipulating sensory input, directly by electrical intracortical microstimulation (ICMS). They found that in adult cat, a few hours of local stimulation induced a highly variable complex and nonlocal reorganization of the orientation map. Previously it was reported that by using a pairing protocol combining ICMS with simultaneous visual stimulation, the general structure of the orientation map is pre- 
served [79]. However, whereas the former study recorded maps up to 18 hours after ICMS, the latter only addressed a time window up to $3-4$ hours after ICMS suggesting that changes may develop on a slow temporal scale. To study the longterm behavior of the map after ICMS and to analyze in detail the processes of reorganization, a novel study was initiated combining ICMS with chronic experiments accompanied by a theoretical analysis of the underlying process. The results of this study are presented in Chapters 7 and 8 .

\subsection{The self-organizing orientation map}

Viewed from a dynamical systems perspective, the activity-dependent remodeling of the cortical network described above is a process of dynamical pattern formation. In this picture, spontaneous symmetry breaking in the developmental dynamics of the cortical network underlies the emergence of cortical selectivities such as orientation preference [61]. The subsequent convergence of the cortical circuitry towards a mature pattern of selectivities can be viewed as the development towards an attractor of the developmental dynamics [107]. This is consistent with the interpretation of cortical development as an optimization process. In the following, we will briefly describe a model [105] that is based on this view. This model is the starting point of a number of analyses carried out in this thesis.

\subsubsection{A pattern formation model}

Self-organization has been observed to robustly produce large scale structures in various complex systems. Often, the class of patterns emerging depends on fundamental system properties such as symmetries rather than on the system specific details. Its formation can therefore be described by an effective model incorporating only these properties. For patterns with a typical scale such a model is

$$
\begin{aligned}
\partial_{t} z & =F[z] \\
& =L_{S H} z+N_{2}[z]+N_{3}[z]+\cdots
\end{aligned}
$$

where the linear part is the Swift-Hohenberg operator [23, 89]

$$
L_{S H}=r-\left(k_{c}^{2}+\nabla^{2}\right)^{2}
$$

and $z(\mathbf{x}, t)$ is a scalar field. If the bifurcation parameter $r<0$, the homogeneous state $z(\mathbf{x})=0$ is stable. For $r>0$, a pattern with average spacing close to $\Lambda=$ $2 \pi / k_{c}$ emerges. The lowest order nonlinearities $N_{2}$ and $N_{3}$ are quadratic and cubic in $z$, respectively. The form of these nonlinearities determines the class of the 
emerging pattern, whether e.g. hexagons, rotating stripes, spiral waves, or of other type.

In analogy to the theory of complex systems Wolf [105] adopted a model of the form Eq. (2.5) with nonlinearities derived from key features of the visual cortex. As in experimental recordings, orientation columns are represented by a complex field [90, 107]

$$
z(\mathbf{x})=|z(\mathbf{x})| e^{i 2 \vartheta(\mathbf{x})}
$$

where $\vartheta$ is the orientation preference and $|z|$ a measure of the selectivity at location $\mathbf{x}$ in the map. The factor 2 in the exponent accounts for the $\pi$-periodicity of stimulus parameter orientation. The model includes the effects of long-range intracortical connections between columns with similar orientation preference (Fig. 2.2). Based on the repetition of columnar circuits across cortex it is assumed that the dynamics is symmetric with respect to translations,

$$
F\left[\hat{T}_{\mathbf{y}} z\right]=\hat{T}_{\mathbf{y}} F[z] \text { with } \hat{T}_{\mathbf{y}} z(\mathbf{x})=z(\mathbf{x}+\mathbf{y}),
$$

and rotations

$$
F\left[\hat{R}_{\beta} z\right]=\hat{R}_{\beta} F[z] \text { with } \hat{R}_{\beta} z(\mathbf{x})=z\left(\left[\begin{array}{ll}
\cos (\beta) & \sin (\beta) \\
-\sin (\beta) & \cos (\beta)
\end{array}\right] \mathbf{x}\right)
$$

of the cortical sheet. This means that patterns that can be converted to one another by translation or rotation of the cortical layers are equivalent solutions of Equation. It is further assumed that the dynamics is symmetric with respect to shifts in orientation,

$$
F\left[e^{i \phi} z\right]=e^{i \phi} F[z]
$$

such that patterns whose arrangement of iso-orientation domains is the same but whose orientation preference values differ by a given amount, are equivalent solutions of Equation. Solutions contain equal representation of all stimulus orientations. Moreover, we neglect all possible couplings to other visual cortical representations such as ocular dominance or retinotopy. Considering only leading order terms up to cubic nonlinearities the nonlinear part reads [105]

$$
\begin{aligned}
N_{3}[z(\mathbf{x})]= & (g-1)|z(\mathbf{x})|^{2} z(\mathbf{x})+ \\
& (2-g) \int d^{2} \mathbf{y} K_{\sigma}(\mathbf{y}-\mathbf{x})\left(z(\mathbf{x})|z(\mathbf{y})|^{2}+\frac{1}{2} \bar{z}(\mathbf{x}) z(\mathbf{y})^{2}\right)
\end{aligned}
$$

and $N_{2}=0$. Long-range interactions are mediated through convolutions of a Gaussian

$$
K_{\sigma}(\mathbf{x})=\frac{1}{2 \pi \sigma^{2}} e^{-\frac{\mathbf{x}^{2}}{2 \sigma^{2}}}
$$

with SD $\sigma$ of the order of column spacing $\Lambda$. The second parameter $0 \leq g \leq 2$ controls local and nonlocal influences. The model minimizes an energy functional during development. It is consistent with synaptic models based on Hebb-type plasticity, e.g. [32, 62, 92]. 


\subsubsection{Weakly nonlinear analysis}

Close to the finite wavelength instability (at $r \ll 1$ ), stationary solutions to Eq. (2.5) with (2.6) and (2.11) can be calculated analytically using a perturbation method called weakly nonlinear stability analysis. When the dynamics is close to a finite wavelength instability, the essential Fourier components of the emerging pattern are located on the critical circle. Thus, solutions may be sought in terms of Planform patterns

$$
z(\mathbf{x})=\sum_{j} A_{j} e^{i \mathbf{k}_{j} \mathbf{x}}
$$

composed of a finite number of Fourier components. By symmetry, the dynamics of amplitudes $A_{i}$ of a planform are governed by amplitude equations

$$
\dot{A}_{i}=r A_{i}-\sum_{j} g_{i j}\left|A_{j}\right|^{2} A_{i}-\sum_{j} f_{i j} A_{j} A_{j^{-}} \bar{A}_{i^{-}}
$$

where $j^{-}$denotes the index of the mode antiparallel to mode $j$. The form of Eq. (2.13) is universal for models of a complex field $z$ satisfying symmetry assumptions 2.8 2.10). All model dependences are included in the coupling coefficients $g_{i j}$ and $f_{i j}$ and may be obtained from $F[z]$ by multiscale expansion [23, 57]. Denoting the angle between the wave vectors $\mathbf{k}_{i}$ and $\mathbf{k}_{j}$ by $\alpha$, the coefficients read

$$
\begin{aligned}
g_{i j} & =\left(1-\frac{1}{2} \delta_{i j}\right) g(\alpha) \\
f_{i j} & =\left(1-\delta_{i j}-\delta_{i^{-}}\right) f(\alpha)
\end{aligned}
$$

where

$$
\begin{aligned}
& g(\alpha)=g+2(2-g) \exp \left(-\sigma^{2} k_{c}^{2}\right) \cosh \left(\sigma^{2} k_{c}^{2} \cos (\alpha)\right) \\
& f(\alpha)=\frac{1}{2} g(\alpha)
\end{aligned}
$$

are called angle-dependent interaction functions.

Stationary solutions of Eq. 2.14) are given by planforms

$$
z(\mathbf{x})=\sum_{j=0}^{n-1}\left|A_{j}\right| e^{i\left(l_{j} \mathbf{k}_{j} \mathbf{x}+\phi_{j}\right)}
$$

of order $n$ with wavevectors

$$
\mathbf{k}_{j}=k_{c}\left(\cos \left(\frac{j \pi}{n}\right), \sin \left(\frac{j \pi}{n}\right)\right)
$$




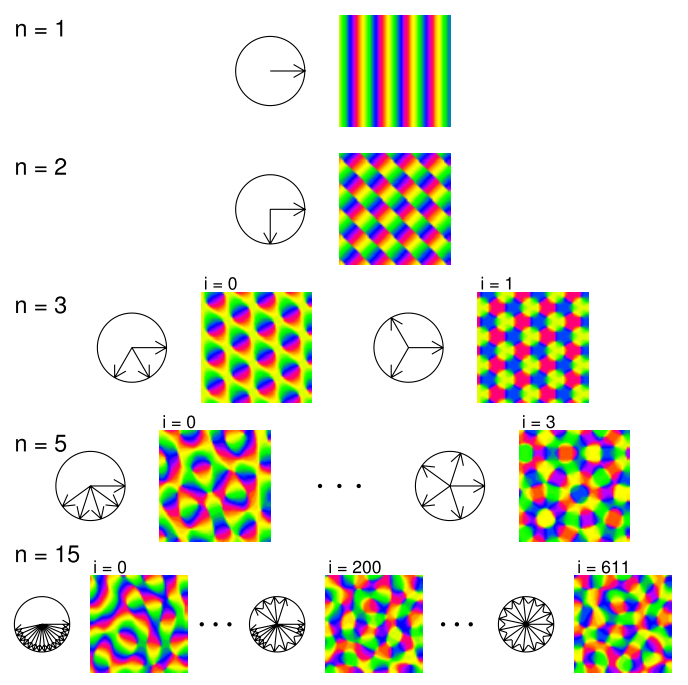

Figure 2.4: Essentially complex planforms with different numbers $n=$ $1,2,3,5,15$ of active modes: The patterns of orientation preferences $\theta(\mathbf{x})$ are shown. The diagrams to the left of each pattern display the position of the wavevectors of active modes on the critical circle. For $n=3$, there are two patterns; for $n=5$, there are four; and for $n=15$, there are 612 different patterns.

distributed equidistantly on the upper half of the critical circle and binary values $l_{j}= \pm 1$ determining whether the mode with wave vector $\mathbf{k}_{j}$ or with wavevector $-\mathbf{k}_{j}$ is active. These planforms cannot realize a real valued function and are called essentially complex planforms (Fig. 2.4). For such planforms the third term in Eq. (2.14) vanishes and the effective amplitude equations for the active modes reduce to a system of Landau equations

$$
\dot{A}_{i}=r A_{i}-\sum_{j} g_{i j}\left|A_{j}\right|^{2} A_{i}
$$

with stationary solutions 2.17 with amplitudes of equal modulus

$$
\left|A_{i}\right|=\sqrt{\frac{r}{\sum_{j} g_{i j}}}
$$

and an arbitrary phase $\phi_{i}$ independent of the mode configuration $l_{j}$. If the dynamics is stabilized by long-range nonlocal interactions $(g<1, \sigma>\Lambda)$, large $n$ planforms are the only stable solutions. In this long-range regime, the order $n$ grows linearly with the interaction range $2 \pi \sigma / \Lambda$. For a given order $n$, different planforms are degenerated in energy. This is a consequence of a fourth symmetry of the nonlinear part 2.11) namely the permutation symmetry

$$
N_{3}(u, v, w)=N_{3}(w, u, v) .
$$

This symmetry implies that the relevant stable solutions are essentially complex planforms which in turn guarantees that all stimulus orientations are represented in equal parts. The property of multistability is characteristic for this model class and will play an important role in Chapter 7 


\subsubsection{Pinwheel density}

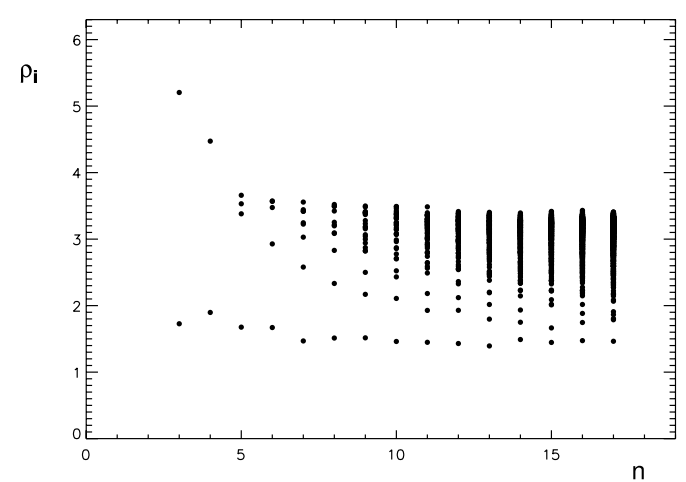

Figure 2.5: Pinwheel densities of the constitutively different essentially complex planforms for $n=3,4, \ldots, 17$. In the case of $n=3$, a periodic pattern is formed in which the pinwheel densities are exactly equal to $2 \cos (\pi / 6) \simeq 1.73$ and $6 \cos (\pi / 6) \simeq 5$.2. The points mark the numerically determined pinwheel densities $\rho_{i}$ of the constitutively different planforms for different numbers of active modes $n$. With increasing numbers of active modes the pinwheel densities occur within an interval of allowed pinwheel densities $1.4<\rho<3.5$.

Whereas degenerate in energy, essentially complex planforms of order $n$ vary substantially in their pinwheel densities. Fig. 2.5 shows the pinwheel densities $\rho_{i}$, i.e. the number of pinwheels per unit area $\Lambda^{2}$, of the essentially complex planforms from Fig. 2.4 of various order $n$. Each value represents an average over the ensemble of phases $\phi_{j}$. Pinwheel densities fill a band of values between 1.5 and 3.5 with the majority of values between 2 and 3.5. Pinwheel density selection is analyzed in experimental data in Chapter 4 , and in the described model in Chapters 5 and 6. 


\section{Interareal coordination of column development in the visual cortex}

\subsection{Motivation}

It is a necessary prerequisite for calculating pinwheel densities to determine the column spacing $\Lambda$ with high precision. Owing to its definition, $\rho=\hat{\rho} \Lambda^{2}$ where $\hat{\rho}$ is the pinwheel density in units $1 / \mathrm{mm}^{2}$, the column spacing $\Lambda$ contributes quadratically to the pinwheel density $\rho$. A relative error in $\Lambda$, therefore, results in a relative error twice as large for $\rho$. Furthermore, since there is no apriory reason to assume that the column spacing is constant within a map, it is important to estimate column spacings locally. Visual inspection of orientation maps suggests a considerable variation of local column spacing at least in some cases.

In this chapter, we investigate the column spacing locally within a set of complete visual areas V1 and V2 in cat visual cortex, both containing a complete representation of the binocular contralateral visual field [97 98]. We adopt methods that we used previously for an analysis of genetic influence on columns spacing [44, 45] and calculate a map of local column spacing $\Lambda(\mathbf{x})$ for each orientation map expressing the spatial variation of local column spacing in this map. Decomposing $\Lambda(\mathbf{x})$ into the three components average, systematic variation, and individual variation reveals that in V1 and V2 and in the left and right brain hemisphere, column spacings covary in regions representing the same part of the visual field. Surprisingly, this matching of column spacing even applies to individual brains and consolidates with age. The analysis demonstrates the precision of the estimation of local column spacings.

\subsection{Methods}

\subsubsection{Animals}

We analyzed 2-deoxyglucose (2-DG) labeled patterns of orientation columns in the visual cortex (V1 and V2) of 27 normally reared cats (41 hemispheres). From this group, 20 animals (31 hemispheres) were born in the animal house of the Max-Planck-Institut für Hirnforschung in Frankfurt am Main, Germany, 7 animals 
(10 hemispheres) were bought from two animal breeding companies in Germany (Ivanovas, Gaukler). All animals stayed at the animal house until the 2-DG experiments. The visual stimuli during the 2-DG experiments were always identical in spatial and temporal frequency, and only differed in orientation. Mostly cardinal orientations were used.

\subsubsection{Image processing}

Photoprints of the 2-DG autoradiographs were digitized using a flat-bed scanner (OPAL ultra, Linotype-Hell AG, Eschborn, Germany, operated using Corel Photoshop) with an effective spatial resolution of 9.45 pixels/mm cortex and 256 grey levels per pixel. For every autoradiograph this yielded a two-dimensional (2D) array of grey values $I_{0}(\mathbf{x})$, where $\mathbf{x}$ (a $2 \mathrm{D}$ vector) is the position within the area and $I_{0}$ its intensity of labeling. For every autoradiograph we defined two regions of interest (ROI) encompassing the patterns labeled in areas V1 and V2. The manually defined polygons encompassing the entire patterns of orientation columns within areas V1 and V2, respectively, were stored together with every autoradiograph. Only the patterns within areas V1 and V2 were used for subsequent quantitative analysis. Regions with very low signal and minor artefacts (scratches, folds, and air bubbles) were excluded from further analysis. All digitized patterns were highpass filtered using the Gaussian kernel $K(\mathbf{y})=\frac{1}{2 \pi \sigma_{K}^{2}} \exp \left(-\mathbf{y}^{2} / 2 \sigma_{K}^{2}\right)$ with a spatial width of $\sigma_{K}=0.43 \mathrm{~mm}$ for $\mathrm{V} 1$ and $\sigma_{K}=0.57 \mathrm{~mm}$ for V2. The patterns were then centered to yield $\int_{V 1} d^{2} y I(\mathbf{y})=0$. To remove overall variations in labeling intensity, patterns from V2 were thresholded to uniform contrast by setting $I(\mathbf{x})=1$ in regions larger than 0 , and $I(\mathbf{x})=-1$ in regions smaller than 0 . Finally, values in artefact regions and in regions outside of areas V1 and V2 were set to zero.

\subsubsection{Spacing analysis}

Patterns of orientation columns were analyzed using a wavelet method introduced recently [44, 45]. For each analyzed pattern of orientation columns we determined a 2D map representing the column spacing at each cortical location. We first calculated wavelet representations of a given pattern $I(\mathbf{x})$ by

$$
\hat{I}(\mathbf{x}, \theta, l)=\int_{A} d^{2} y I(\mathbf{y}) \psi_{\mathbf{x}, \theta, l}(\mathbf{y}),
$$

where $\mathbf{x}, \theta, l$ are the position, orientation, and scale of the wavelet $\psi_{\mathbf{x}, \theta, l}(\mathbf{y}), \hat{I}(\mathbf{x}, \theta, l)$ denotes the array of wavelet coefficients and A denotes the ROI in V1 or V2. We used complex-valued Morlet-wavelets defined by a mother-wavelet

$$
\psi(\mathbf{x})=\exp \left(-\frac{\mathbf{x}^{2}}{2}\right) e^{i \mathbf{k}_{\psi} \cdot \mathbf{x}}
$$


and

$$
\psi_{\mathbf{x}, \theta, l}(\mathbf{y})=l^{-1} \psi\left(\Omega^{-1}(\theta) \frac{\mathbf{y}-\mathbf{x}}{l}\right)
$$

with the $2 \mathrm{D}$ rotation matrix $\Omega$. The characteristic wavelength of a wavelet with scale $l$ is $\Lambda_{\psi} l$ with $\Lambda_{\psi}=2 \pi /\left|\mathbf{k}_{\psi}\right|$. We used wavelets with about 7 lobes, i.e. $\mathbf{k}_{\psi}=$ $(7,0)$, to ensure a narrow frequency representation while keeping a good spatial resolution of the wavelet. From these representations we calculated the orientation averaged modulus

$$
\bar{I}(\mathbf{x}, l)=\int_{0}^{\pi} \frac{d \theta}{\pi}|\hat{I}(\mathbf{x}, \theta, l)|
$$

of the wavelet coefficients for every position $\mathbf{x}$, and then determined the scale

$$
\bar{l}(\mathbf{x})=\operatorname{argmax}(\bar{I}(\mathbf{x}, l))
$$

maximizing $\bar{I}(\mathbf{x}, l)$. The corresponding characteristic wavelength

$$
\Lambda(\mathbf{x})=\bar{l}(\mathbf{x}) \Lambda_{\psi}
$$

was used as an estimate for the local column spacing at the position $\mathbf{x}$. For every position (spatial grid-size $0.12 \mathrm{~mm}$ ) wavelet coefficients for 12 orientations $\theta_{i} \in$ $\{0, \pi / 12, \ldots, 11 \pi / 12\}$ were calculated for V1 on 15 scales $l_{j}$ (with $l_{i} \Lambda_{\psi}$ equally spaced in $[0.5,2] \mathrm{mm}$ ) and for $\mathrm{V} 2$ on 21 scales $l_{j}$ (spaced in $[0.5,2.5] \mathrm{mm}$ ). The scale maximizing $\bar{I}(\mathbf{x}, l)$ was then estimated as the maximum of a polynomial in $l$ fitting the $\bar{I}\left(\mathbf{x}, l_{j}\right)$ for a given position $\mathbf{x}$ (least square fit). The local column spacing was calculated for typically 4 flatmount sections in each hemisphere. Values at corresponding locations in different sections were averaged and combined resulting in a single map of local column spacing $\Lambda(\mathbf{x})$ for V1 and V2 in each brain hemisphere. Locations sampled by $<2$ sections were excluded from further analysis. After superposition, the local column spacing $\Lambda(\mathbf{x})$ was smoothed using a Gaussian kernel with $\sigma=1.25 \mathrm{~mm}$.

For every map of local column spacing $\Lambda(\mathbf{x})$, the mean column spacing $\Lambda=$ $\langle\Lambda(\mathbf{x})\rangle_{\mathbf{x}}$ was calculated. It measures whether a pattern predominantly contains large or small orientation columns. The map of the systematic topographic variation of column spacing $\Lambda_{\text {sys }}(\mathbf{x})$ was obtained by $\Lambda_{\text {sys }}(\mathbf{x})=\langle\Lambda(\mathbf{x})-\Lambda\rangle_{\text {hemis }}$, i.e. by subtracting from each map of local column spacing $\Lambda(\mathbf{x})$ its mean value $\Lambda$ and then superimposing and averaging over different hemispheres. For superposition, we localized the representations of the vertical meridians (VM) and the areae centrales on the autoradiographs and aligned the 2D maps of local column spacing from different animals using these landmarks [45, 52]. Maps from right hemispheres were mirror inverted. The alignment of spacing maps based on these landmarks matches corresponding locations from different hemispheres. The systematic topographic variation of column spacing $\Lambda_{s y s}(\mathbf{x})$ was calculated only at locations $\mathbf{x}$ 
where at least 8 hemispheres contributed. Maps of individual topographic variation of column spacing, $\Lambda_{\text {indv }}(\mathbf{x})$, were obtained by $\Lambda_{\text {indv }}(\mathbf{x})=\Lambda(\mathbf{x})-\Lambda-\Lambda_{\text {sys }}(\mathbf{x})$, i.e. by subtracting from each map of local column spacing its mean column spacing $\Lambda$ and the map of systematic topographic variation $\Lambda_{\text {sys }}(\mathbf{x})$.

\subsubsection{Accuracy and measurement errors}

All quantities presented are subject to measurement errors. The estimation of measurement errors was carried out following [45]. The error of the local colum spacing, $\Delta \Lambda$, and the error of the mean column spacing, $\Delta \Lambda(\mathbf{x})$, were estimated based on the multiple flatmount sections analyzed for every hemisphere. Spacing values were calculated for every section individually and SEM were estimated from the values for different sections. SEMs for mean column spacings $\Lambda$ were $15 \mu \mathrm{m}$ for V1 and $35 \mu \mathrm{m}$ for V2. Errors were larger in V2 due to its smaller size and the weaker labeling. Errors $\Delta \Lambda(\mathbf{x})$ of the local column spacing were on average $58 \mu \mathrm{m}$ in V1 and $64 \mu \mathrm{m}$ in V2. The error of the systematic topographic variation $\Lambda_{s y s}(\mathbf{x})$ was calculated by error propagation from the error of the local column spacing $\Lambda(\mathbf{x})$, that is $\Delta \Lambda_{\text {sys }}(\mathbf{x})=\sqrt{\left\langle\Delta \Lambda(\mathbf{x})^{2}\right\rangle_{\text {hemis }}} / \sqrt{N_{\text {hemis }}}$, where the average is taken over the population of the $N_{\text {hemis }}$ hemispheres contributing to $\Lambda_{s y s}(\mathbf{x})$. Its error was relatively small (SEM, $19 \mu \mathrm{m}$ for V1, and $23 \mu \mathrm{m}$ for V2). The maps of individual topographic variation were mainly inflicted by the error of local column spacing and the systematic topographic column spacing.

\subsubsection{Decomposition of variance}

The variances of all spacing parameters $\left(\right.$ e.g $\left.\Lambda(\mathbf{x}), \Lambda, \Lambda_{\text {sys }}(\mathbf{x})\right)$ were error corrected following [45]. The variance $v_{\text {mean }}$ of the mean column spacing $\wedge$ was calculated by $v_{\text {mean }} \approx s_{\text {mean }}^{2}-\left\langle\Delta \Lambda^{2}\right\rangle_{\text {hemis }}$, where $s_{\text {mean }}$ is the SD of the values of the mean column spacing $\Lambda$ for different animals and $\left\langle\Delta \Lambda^{2}\right\rangle_{\text {hemis }}$ is the squared error of $\Lambda$ averaged over hemispheres from all animals. For its square root $\sqrt{\left\langle\Delta \Lambda^{2}\right\rangle_{\text {hemis }}}$ we obtained $0.018 \mathrm{~mm}$ for $\mathrm{V} 1$ and $0.046 \mathrm{~mm}$ for V2. The variance $v_{\text {sys }}$ of the systematic intraareal variability of local column spacing was calculated from the SD $s_{s y s}$ of the systematic topographic variation $\Lambda_{s y s}(\mathbf{x})$ and the its error $\Delta \Lambda_{s y s}(\mathbf{x})$ by $v_{s y s} \approx s_{s y s}^{2}-\left\langle\Delta \Lambda_{s y s}(\mathbf{x})^{2}\right\rangle_{\mathbf{x}}$. The square root of the spatially averaged squared error, $\sqrt{\left\langle\Delta \Lambda_{\text {sys }}(\mathbf{x})^{2}\right\rangle_{\mathbf{x}^{\prime}}}$ yielded $0.020 \mathrm{~mm}$ for $\mathrm{V} 1$ and $0.024 \mathrm{~mm}$ for $\mathrm{V} 2$, respectively. The variance $v_{\text {all }}$ of all orientation column spacings in all hemispheres (from V1 or from $V 2$, respectively) is given by $v_{\text {all }}=s_{\text {all }}^{2}-\left\langle\Delta \Lambda(\mathbf{x})^{2}\right\rangle_{\text {all }}$, where $\sqrt{\left\langle\Delta \Lambda(\mathbf{x})^{2}\right\rangle_{\text {all }}}$ is the square root of the error of the local spacing squared and averaged over all locations in all hemispheres. For V1 we obtained 0.088mm, for V2 $0.093 \mathrm{~mm}$. Denoted 
by $s_{\text {all }}$ is the SD of local spacing values $\Lambda(\mathbf{x})$ from all hemispheres.

The total variance $v_{\text {all }} \approx v_{\text {mean }}+v_{\text {sys }}+v_{\text {indv }}$ is composed of the variance of the mean column spacing $v_{\text {mean }}$, the variance of the systematic topographic variation of column spacing $v_{s y s}$, and the average variance of the individual topographic variation of column spacings $v_{i n d v}$. This decomposition provides an estimate for the relative magnitudes of the different contributions to the total variance in the population of column spacing maps from V1 or V2 (Fig. 3.1 d).

\subsubsection{Morphing}

Column spacing maps from V2 were morphed on those from V1 by thin-plate spline interpolation. By this method, defined reference points in V2 were morphed on corresponding points in V1, and the remaining locations are morphed such that the distortion of the morphed map is minimal. We used 30 reference points in areas $\mathrm{V} 1$ and V2 distributed along the common V1/V2 border, and along the lateral border of V2 and the medial border of V1. The same morphing was used for all V1/V2 pairs. This provides only a rough mapping of corresponding locations in individual V1/V2 pairs (see e.g. the pronounced size variation of V1 [44]. No attempt was made to optimize the similarity of spacing maps of V1/V2 pairs.

\subsubsection{Permutation tests}

Permutation tests were used to test for statistical significance. In these tests the value of a statistic (e.g. for cross-correlation or for an average differences) was compared to values obtained for randomized data. Usually, a distribution of $10^{4}$ random realizations was sampled. The significance value is given by the probability of obtaining the real value or a value more extreme by chance. The significance value for the correlation between mean column spacings $\Lambda$ in V1 and V2 was calculated by permuting all mean column spacings from $\mathrm{V} 2$ and is given by the fraction of correlation coefficients found to be larger than the real value. The significance of the distance $\Delta$ between the map of the residual topographic variation from V1 and and the morphed map from V2 was calculated by permuting among all maps from V2. The average distance $\Delta$ was calculated from all V1/V2 pairs with a common area of at least $70 \mathrm{~mm}^{2}$ (in the coordinate system of V1) and compared to averages obtained in $10^{4}$ comparable groups of pseudo V1/V2 pairs. The significance value is given by the fraction of averages smaller than the average of the real distance $\Delta$. Distances $\Delta$ between individual topographic variations in the left and right hemispheres were compared to pseudo left/right pairs generated from all hemispheres. All significance tests regarding the distance $\Delta$ were based on aged matched randomizations. Cases 9 weeks old or younger $(n=19)$ were exchanged by pseudo pairs generated from this group only. Random pairs older than 9 weeks were gen- 
erated only from the cases older than 9 weeks $(n=22)$.

\subsection{Decomposition of column spacing}

We analyzed the spacing of orientation columns in V1 and V2 in $n=41$ brain hemispheres ( $\mathrm{N}=27$ animals) using the wavelet method described in [44]. Because this method provides highly precise estimates of local column spacing with an error much smaller than the large intrinsic variability of column spacings (SEM, 15$50 \mu \mathrm{m})$, differences and similarities of column spacings in the sample can be identified reliably. The spacing of adjacent orientation columns was calculated independently at every cortical location in each area (Fig. 3.1p). Thus, for each area a two-dimensional map of local column spacing was calculated representing the variability of local column spacings in this area.

\subsection{Mean column spacing}

From these maps we first extracted the mean column spacing in areas V1 and V2 for each hemisphere and assessed their statistical dependence (Fig. 3.2). We found that mean column spacings $\Lambda$ varied considerably in different individuals (Fig. 3.2 .). In V1, values ranged between $1.1 \mathrm{~mm}$ and $1.4 \mathrm{~mm}$, in $\mathrm{V} 2$ between $1.2 \mathrm{~mm}$ and $1.8 \mathrm{~mm}$. The distributions for the two areas were partially overlapping with the smallest column spacings from V2 at about the average value of V1. Nevertheless, in all hemispheres, the mean column spacing $\Lambda$ in V2 was substantially larger than in V1, consistent with previous reports [50, 52]. Mean column spacings $\Lambda$ did not vary independently across different animals in V1 and V2, but were substantially correlated in both areas (Fig 3.2 d; r=0.62, $\mathrm{p}<10^{-5}$, permutation test).

\subsection{Systematic topographic variation}

In both areas V1 and V2, orientation columns generally exhibited a substantial intra-areal variation in spacing around the mean column spacing. In each area, one part of this variation is common to all hemispheres. In the following, we will call this component the systematic topographic variation of column spacings (the blue map in Fig. 3.1k). It is the intra-areal variation (in V1 or V2) averaged over the entire population of hemispheres (i.e. a 2D spacing map with zero mean). For averaging, the V1/V2 borders of different hemispheres were aligned and maps from right hemispheres were mirror-inverted. The remaining part of the variation characterizes an individual hemisphere. Accordingly, this part will be called individual topographic variation of column spacings (orange map in Fig. 3.1k). It is also 

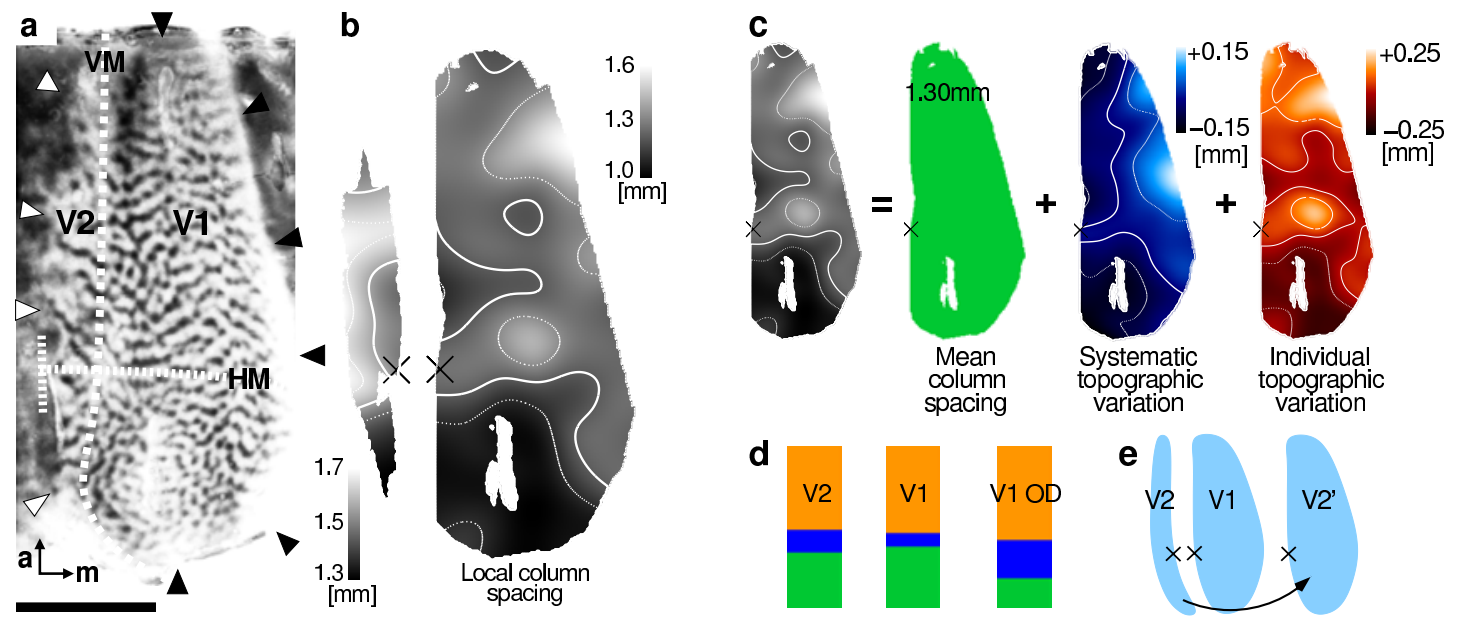

Figure 3.1: Quantification of orientation column spacing in cat visual cortex. a, Overall layout of 2-[ $\left[{ }^{14} \mathrm{C}\right]$-deoxyglucose labeled (dark grey) orientation columns in flat mount sections of areas V1 (right) and V2 (left). Black and white arrow heads indicate the external border of V1 and V2, respectively. Cortical representations of the vertical meridian (VM) (i.e. the V1/V2 border) and the horizontal meridian (HM) of the visual field are represented by the white dashed lines (a=anterior, $\mathrm{m}=$ medial; scale bar, $10 \mathrm{~mm}$ ). b, 2D-maps of local column spacing in areas V2 (left) and V1 (right) (grey scale coded). Contour lines are drawn at the mean spacing (thick white line) and mean $\pm \mathrm{SD}$ (thin white lines). Black crosses mark the central visual field representation. c, Decomposition of the V1-spacing map in $\mathbf{b}$ : Each map of local column spacing (grey) is composed of (i) the mean column spacing (green) (ii) the systematic part of the topographic variation of local column spacing (population averaged, blue), and (iii) the individual part of topographic variation (orange). d, According to c, the variance of all column spacings in the population is the sum of (i) the variance of the mean column spacings of the different areas (green), (ii) the variance of the systematic variation (blue), and (iii) the average variance of the individual variation (orange). The percentages of these variance components are represented by colored bars for V2 (i, 38\%; ii, 8\%; iii, 54\%) and V1 (i, 34\%; ii, 14\%; iii, 52\%), and for ocular dominance columns in cat V1 (i, 18\%; ii, 24\%; iii, 58\%). e, For comparing layouts in V1 and V2, V2 spacing maps were mirror inverted and morphed (shown schematically) aligning regions representing similar parts of the visual field in areas V1 and V2.

a 2D spacing map with zero mean calculated by subtracting the first two components from the map of local column spacing (grey map in Fig. 3.1.). The variances of the systematic and the individual topographic variations add up together with the variance of mean column spacings to the total variance of column spacings in the sample. Interestingly, the individual topographic variation accounted for the largest part of the variance of column spacings in both areas V1 and V2 (Fig. 3.1 d).

The systematic topographic variation of column spacings in V1 exhibited virtually the same overall 2D organization as the one in V2, appearing as a horizontally stretched mirror-image of the V2 map when displayed side by side (Fig. 3.3, a and 

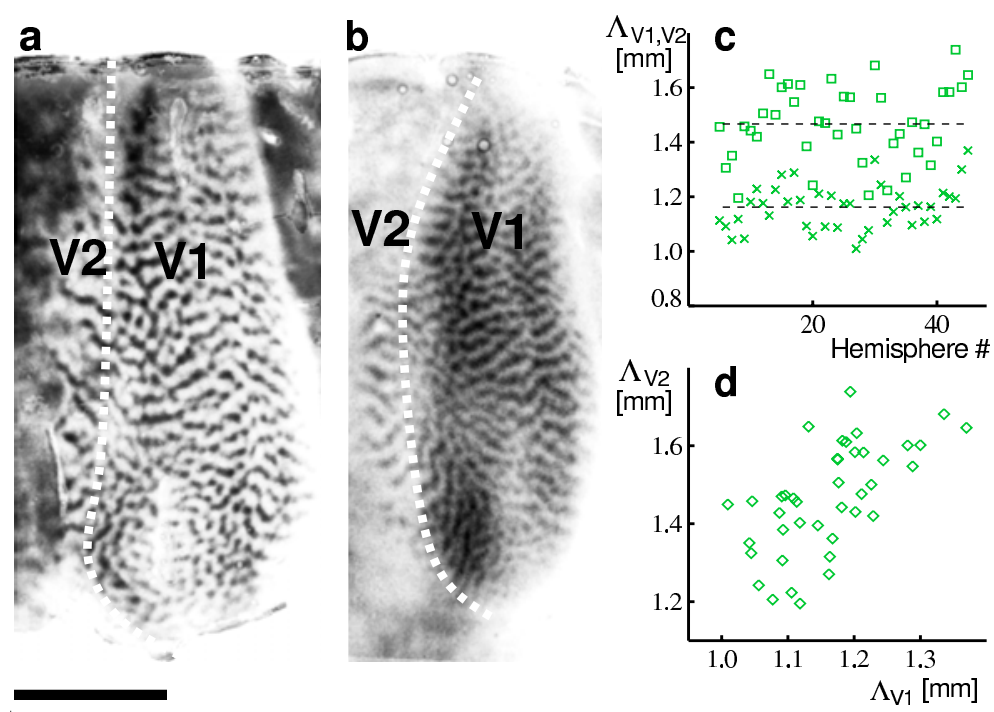

Figure 3.2: Mean column spacings in V1 and V2 covary. $\mathbf{a}, \mathbf{b}$, Overall layout of orientation columns in V1 and V2 in two individuals (white dashed line: V1/V2 border; scale bar, $10 \mathrm{~mm}$ ). For $\mathbf{a}$ the mean column spacing $\Lambda$ is relatively large in both areas $(\mathrm{V} 1,1.21 \mathrm{~mm}$; $\mathrm{V} 2,1.58 \mathrm{~mm})$, whereas for $\mathbf{b}$ it is small in both areas $(\mathrm{V} 1,1.09 \mathrm{~mm}$; V2, $1.31 \mathrm{~mm})$. $\mathbf{c}$, Mean column spacings $\Lambda$ in $\mathrm{V} 1$ (crosses) and $\mathrm{V} 2$ (boxes) from $\mathrm{n}=41$ hemispheres ( $\mathrm{N}=27$ animals). Values in $\mathrm{V} 1$ and $\mathrm{V} 2$ vary considerably in different hemispheres (V1,1.0-1.4mm; V2, 1.2-1.8mm). d, Mean column spacings $\Lambda$ in $\mathrm{V} 1$ and $\mathrm{V} 2$ of individual hemispheres are strongly correlated $\left(r=0.62, p<10^{5}\right.$, permutation test).

b): In both areas, columns were systematically wider than average along the representation of the horizontal meridian (HM) with this tendency increasing towards the periphery. In contrast, columns smaller than average more often occurred along the peripheral representations of the vertical meridian (VM). The systematic variation in areas V1 and V2 ranged between $-0.15 \mathrm{~mm}$ and $+0.15 \mathrm{~mm}$ (Fig. 3.3, a and b). In order to conveniently compare topographically corresponding parts in areas V1 and V2, the V2 map was mirror-inverted and morphed by superimposing major landmarks such as the representations of the VM (located along the V1/V2 border), the central visual field, and the HM (Fig. 3.1k, Fig. 3.3k). Since the morphed V2 map strongly resembled the V1 map (compare Fig. 3.3. b and c) the cross-correlation between the maps was high $(\mathrm{r}=0.66)$. Furthermore, the systematic variation observed in a comparable data set of ocular dominance column spacings in cat V1 (Fig. $3.3 \mathrm{~d}$, modified from [45]) also exhibited a very similar intra-areal organization with a strong cross-correlation of $r=0.82$ to the population averaged spacing map for orientation columns in V1 (compare Fig. 3.3, $b$ and $d$ ). 

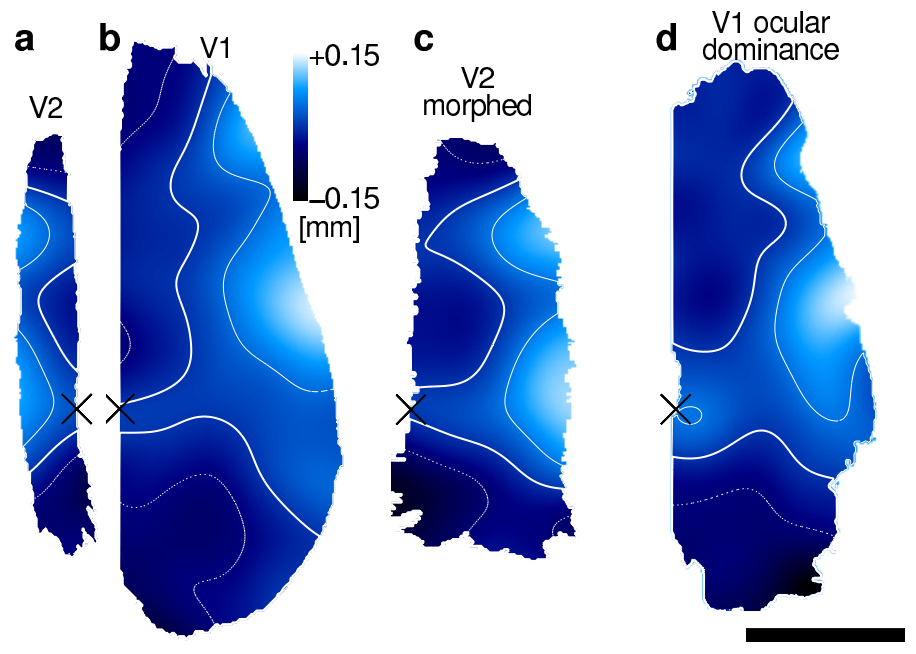

Figure 3.3: The systematic topographic variation of column spacing is similar in subregions representing the same visual field position in areas V1 and V2. a, b, Systematic topographic variation of orientation column spacing in V2 (a) and V1 (b) (color scale codes systematic variation from the mean value; arrangement and symbols as in Fig. 3.1. c, The morphed map from V2 (a). d, Population averaged spacing of ocular dominance columns in cat V1 (modified from [45]). SD for V2, 0.052mm; V1, 0.047mm; V1 ocular dominance columns, $0.049 \mathrm{~mm}$. Scale bar, $10 \mathrm{~mm}$. Note that for both orientation and ocular dominance maps columns representing the $\mathrm{HM}$ and, in particular, the horizontal periphery were on average wider than columns representing the peripheral parts of the VM. Hence, the systematic variations of orientation columns in V1 and V2 were correlated at topographically corresponding locations (correlation between $\mathbf{b}$ and $\mathbf{c}, r=0.69$ ), as were those of orientation and ocular dominance columns in V1 (correlation between $\mathbf{b}$ and $\mathbf{d}, \mathbf{r}=0.82$ ).

\subsection{Individual topographic organization}

Typically, the individual topographic variation of column spacings in V1 and V2 was also similar in regions analyzing the same part of the visual field. The examples shown in Fig. 3.4 A-c display the same general pattern in both areas V1 and V2 with maxima (white) and minima (dark orange) approximately at corresponding retinotopic locations. Among different individuals the pattern of individual variation differed considerably. To quantify the similarity of the individual topographic variation in V1 and V2 we calculated for each hemisphere the absolute value of the difference between both maps averaged over all analyzed locations, called their mismatch $\Delta_{V 1 V 2}$. Mismatches $\Delta_{V 1 V 2}$ were significantly smaller than values obtained for randomly assigned pseudo V1/V2 pairs $(\mathrm{p}=0.03$, age matched permutation test). Thus, in an individual hemisphere the individual topographic variation of column spacings is adjusted at topographically corresponding locations of both areas. 

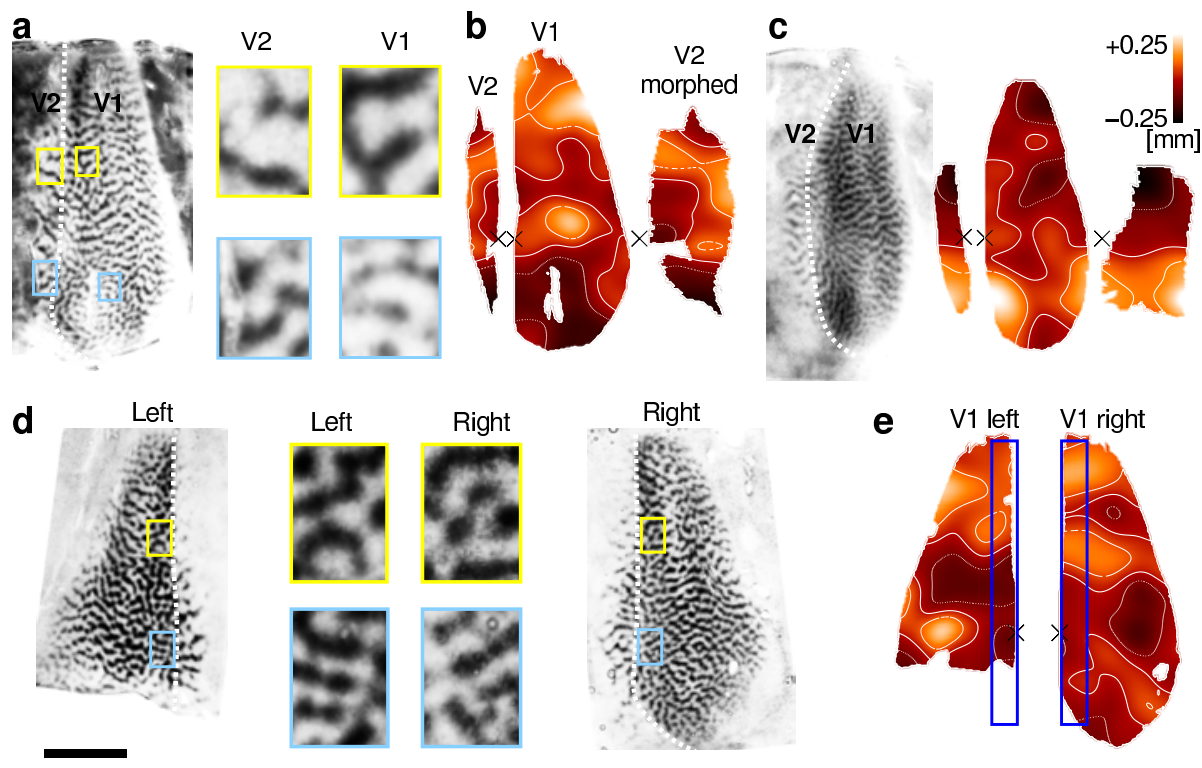

Figure 3.4: In individual brains, columns in different areas are closely matched in size at topographically corresponding subregions. a-c, Similarity of the individual topographic variation of column spacings in V1 and V2. a, The overall layout of orientation columns for the hemisphere shown in Fig. 3.1. A pair of topographically corresponding subregions from the more anterior part of V1 and V2 (yellow boxes) and a pair from the more posterior part (blue boxes) are displayed magnified such that all spacing differences except those due to the individual topographic variation were equalized: Since the relative difference of mean column spacings in V1 and V2 was $\Lambda_{V 2} / \Lambda_{V 1}=1.3$, the subregions from $V 1$ were magnified relative to those from $V 2$ by this factor. To equalize the differences due to the systematic topographic variations the two posterior sub-regions were magnified by an additional factor of $\Lambda_{s y s}\left(\mathbf{x}_{a}\right) / \Lambda_{s y s}\left(\mathbf{x}_{p}\right)=1.05$, where $\Lambda_{s y s}(\mathbf{x})$ is the systematic topographic variation of column spacing and $\mathbf{x}_{a}\left(\mathbf{x}_{p}\right)$ is the center of the anterior (posterior) region of $\mathrm{V} 1$ in the shown hemisphere. Note that the spacing of columns is similar within each pair. $\mathbf{b}$, Patterns of individual variation of column spacing for V2, V1, and the morphed version of $\mathrm{V} 2$ for the hemisphere in a (color scale, black cross and contour lines as in Fig. 3.1. c, Similarity of the individual variation in V1 and V2 at topographically matched locations in another example. d, e, Similarity of the individual topographic variation of column spacings in the left and right brain hemisphere. $\mathbf{d}$, The overall layout of orientation columns in the left and right hemisphere of an individual animal. A pair of topographically corresponding regions from the anterior part of the VM representation in V1 of both hemispheres (yellow boxes) and a pair from a more posterior part in V1 (blue boxes) was magnified such that all differences except the individual topographic variation were equalized (mean column spacings $\Lambda_{V 1}^{r}$ and $\Lambda_{V 1}^{l}$ were equal in both hemispheres and the systematic topographic variations were equalized by magnifying the two posterior sub-regions by a factor $\left.\Lambda_{s y s}\left(\mathbf{x}_{a}\right) / \Lambda_{s y s}\left(\mathbf{x}_{p}\right)=0.98\right)$. Note that the spacing of columns is similar within each pair. $\mathbf{e}$, Patterns of the individual variation of local column spacing in $\mathrm{V} 1$ for the hemispheres in $\mathbf{d}$ displayed with the representation of the VM side by side (crosses and contour lines as in Fig. 3.1. In both residual maps, the blue rectangles (width, 3mm) are positioned at the representation of the VM. Note that the residual maps tend to be similar only along the VM-representations. Scale bar, $10 \mathrm{~mm}$. 
Altogether, these results raise the hypothesis that columns from different areas exhibit an adjusted spacing when they encode a similar visual field position and are synaptically connected. In order to further test this hypothesis we compared columns from V1(V2) in the left and right brain hemisphere encoding similar parts of the VM. Areas V1 and V2 from both hemispheres each contain a full representation of the VM 97 98]. A narrow region of the ipsilateral visual hemifield is represented in both V1 and V2 in the transition zone spanning approximately 1.0$1.5 \mathrm{~mm}$ along the V1/V2 border [68, 69]. V1-columns at corresponding parts of the VM representation in both hemispheres receive similar afferent input and also mutual input mediated by callosal connections concentrated in the vicinity of the VM representation. Our hypothesis predicts that these columns should exhibit matched spacings.

To test this, we analyzed the 2D maps of individual topographic variation of column spacing in V1 within a narrow strip of $3 \mathrm{~mm}$ width adjacent to the V1/V2 border in pairs of left and right hemispheres (Fig. 3.4 $\mathrm{d}-\mathrm{e}$ ). Whereas maps of individual variation from both hemispheres differed at mirror-symmetric locations representing the visual periphery, they were often very similar along the V1/V2 border (Fig. 3.4e). By the mismatch $\Delta_{L R}$ we quantified the absolute value of differences between left and right maps averaged over the defined region. Values of $\Delta_{L R}$ were significantly smaller than those obtained for randomly assigned pairs of hemispheres, $(\mathrm{p}=0.01$, age matched permutation test). A similar behavior was found for V2 (data not shown). Interestingly, $\Delta_{L R}$ were larger for regions more distant from the V1/V2 border. These results further corroborate the specificity of column size matching at topographically corresponding positions in different areas.

\subsection{Consolidation of column size matching}

What is the neuronal mechanism underlying the emergence of coordinated column layouts in widely distributed cortical regions? To our knowledge, there is currently only one scenario supported by experimental evidence that explains our findings. Recently, Hensch and Stryker showed that pharmacologically shifting the balance of inhibition and excitation during development modifies column spacing in cat V1 38]. Presumably, column spacings are also determined by the local inhibitoryexcitatory balance in normal development. Here this balance may be set by regulatory mechanisms, such as synaptic scaling [29], that are sensitive to neuronal activity. The emergence of size matched columns in distant cortical regions might thus be caused by a similar balance of inhibition and excitation in these regions that emerges from their mutual synaptic coupling. Alternatively, one might imagine that the shared visual input of the columns causes the emergence of matched column spacings. However, even major modifications of visual input do not induce 

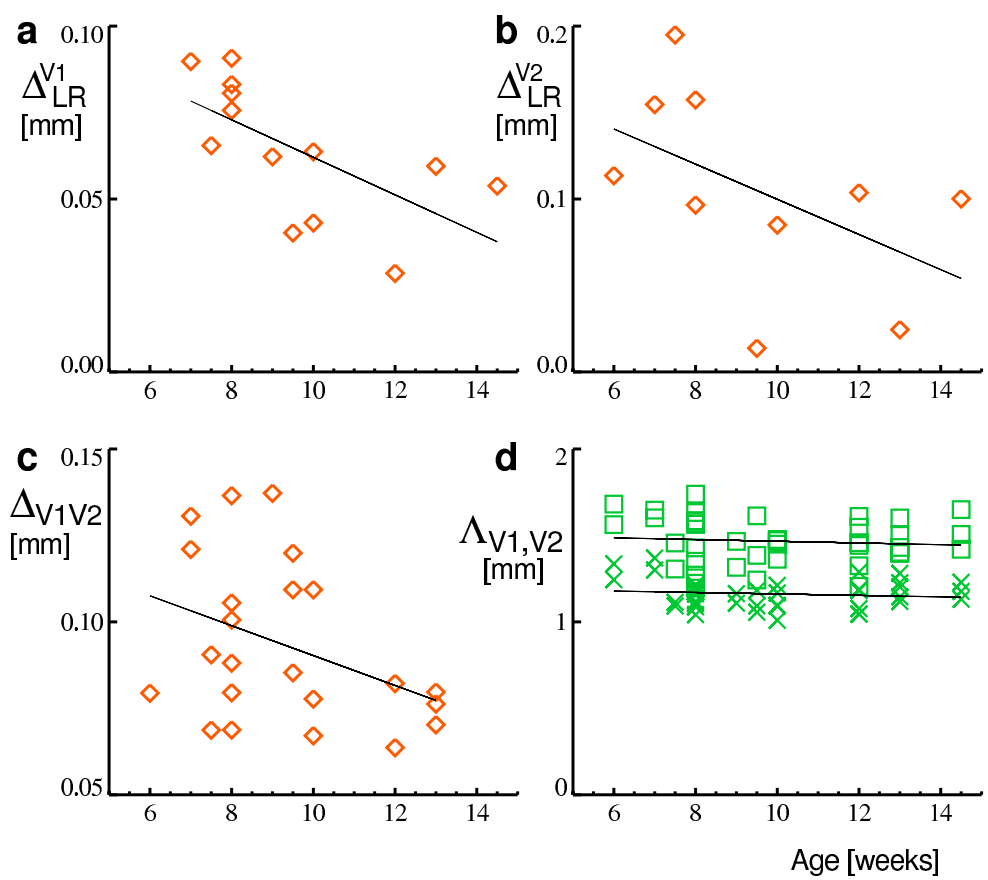

Figure 3.5: Consolidation of column size matching with age. a, Mismatches $\Delta_{L R}$ between the V1 individual topographic variations in the left and right brain hemispheres versus age $(n=13$, calculated within the blue rectangles shown in Fig. 3.4p). b. Mismatches $\Delta_{L R}$ for V2 ( $\mathrm{n}=10$, calculated from the morphed maps of V2). $\mathbf{c}$, Mismatches $\Delta_{V 1 V 2}$ between the individual variations in V1 and in the morphed V2 $\left(n=23\right.$, area larger than $\left.\geq 70 \mathrm{~mm}^{2}\right)$. $d$, Mean column spacings $\Lambda$ in V1 (crosses) and V2 (boxes) (from Fig. 3.2 k) versus age. Correlations with age are significant in $\mathbf{a}(r=-0.64, \mathbf{p}=0.007), \mathbf{b}(r=-0.5, \mathbf{p}=0.02)$ and $\mathbf{c}(r=-0.39, \mathbf{p}=0.01)$, but were not in $\mathbf{d}$. Solid lines are regression lines (least square fit). Note that large mismatches in a-c occur only in young animals but never in those of older age.

detectable changes in column spacing [45, 72] which renders this hypothesis quite unlikely. Because the regulatory mechanisms setting inhibitory-excitatory balance certainly are under genetic control, the balance mechanism described above also offers a simple explanation of the apparently strong impact of genetic factors on column size variability [44, 45].

Our data supports such a dynamical activity-dependent mechanism of column size matching. Fig. 3.5a-c shows the spacing mismatches for topographically corresponding columns in left/right pairs of V1, V2 and for V1/V2 pairs as a function of age. For all three pairs of areas substantial mismatches were only observed in animals younger than 10 weeks. In older animals, mismatches of column spacing were generally less than $0.1 \mathrm{~mm}$. As a consequence all three measures are significantly anti-correlated with animal age $\left(\Delta_{L R}\right.$ for V1 $r=-0.64, \mathrm{p}=0.007$; for V2 $r=-0.5$, $\mathrm{p}=0.02 ; \Delta_{V 1 V 2} r=-0.39, \mathrm{p}=0.01 ;$ age matched permutation test). In contradistinction, 
the average column spacings of areas V1 and V2 are age independent (Fig. 3.5 d). This suggests that the spacings of mutually connected columns are adjusted during development without changing average spacings. The age range in which we observe a decreasing column size mismatch represents the late phase of the critical period for ocular dominance plasticity, which in cats extends into the third month of life. This further supports the hypothesis that activity-dependent developmental mechanisms mediate column size matching.

\subsection{Conclusion}

We analyzed the local spacing of columns in complete orientation maps from cat visual cortex. We compared local column spacings in i) left/right pairs of brain hemispheres and ii) in areas V1 and V2 of individual brain hemispheres. Whereas the size of columns varied strongly within all areas considered, columns in different areas were typically closely matched in size if they were synaptically connected. Exceptional deviations from column size matching were only observed in young animals, suggesting that the layout of connected columns is progressively adjusted over the course of development. The observed matching of column spacing in remote, but synaptically connected regions indicates that the pronounced variation of column spacing is real and not just due to a measurement error. Furthermore, this demonstrates that local column spacing can be measured with high precision.

The formation of cortical columns is often conceptualized as a local process in which synaptic microcircuits confined to the volume of the emerging column are established and selectively refined [33, 46]. However, many neurons that are wiring up locally are simultaneously building macroscopic circuits spanning widely distributed brain regions such as different cortical areas or the two brain hemispheres [77 78]. Thus, in principle, interareal interactions may shape the local column layout. Comparing the layout of distant columns that are mutually connected and represent related aspects of the sensory input, we showed that the columnar architectures of different areas of the cat visual cortex in fact develop in a coordinated manner, not adequately described as a local process.

It is tempting to hypothesize that the interareal coordination of column layouts found here reflects a tight coupling of circuit development and cortical function. Many lines of evidence indeed suggest that the operations of cortical columns in the brains of behaving animals are often inseparably coupled by top down and lateral interactions in far reaching cortical circuits [2, 76, 95]. If activity coordinated between areas is in fact a predominant mode of cortical function, interareal interactions most likely also play a role in the development of cortical columns. 


\section{Pinwheel density selection in the visual cortex}

\subsection{Introduction}

The organization of orientation columns into pinwheel-like patterns has been observed in diverse species such as galagos (primate) [109], ferrets (carnivora) [17] 103], and tree shrews (scandentia) [13] (Fig. 4.1 A-c). These mammals are separated for more than 30 million years of evolution and specialized to different ecological niches with different behavioral rhythms. Their visual systems differ in many respects including the distribution of retinal ganglion cells, the input to the visual cortex and the average orientation selectivity of neurons [27, 43, 48, 73]. It is therefore conceivable that the organization of orientation maps differs strongly in these species. A characteristic quantity for orientation maps is the pinwheel density. It has been suggested that from the pinwheel density in adult visual cortex, the possible developmental process can be inferred [107]. Large inter-species differences of pinwheel densities have been stated previously [64, 66, 92, 107]. However, quantitative conclusions in these studies were difficult, because of the interspecies 64, 66 and intraareal [13, 85] variation of column spacing and the large contribution of noise in the data.

In this chapter, we quantitatively characterize the spatial organization of orientation pinwheels in galagos, ferrets, and tree shrews. We develop a novel pinwheel analysis method that is independent of the spacing of columns and largely robust against noise. We calculate the density and the spatial variation of pinwheels in galagos, ferrets and tree shrews and find that their statistics is universal in all three species.

\subsection{Methods}

To characterize visual cortical orientation maps, we first analyzed the number of pinwheels per unit area. Whereas pinwheel localization requires recording methods providing high signal to noise ratio and spatial resolution, pinwheel counting is relatively robust against imperfect assessment. The reason for this is that by moderate signal inference pinwheels are not eliminated, but only altered in their form and location, a property called topological stability. Noise can annihilate pin- 


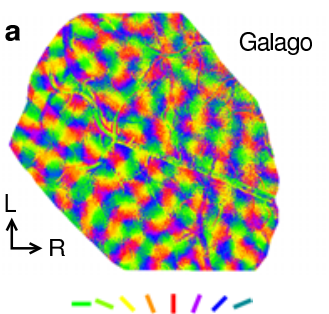

C

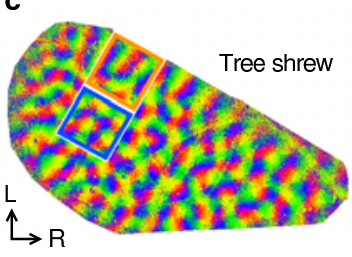

b

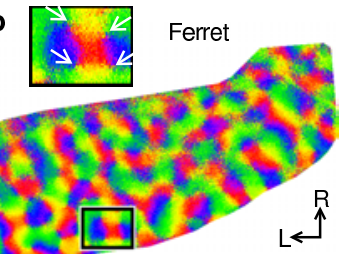

d

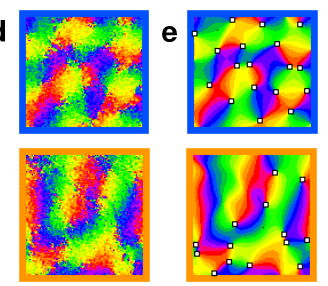

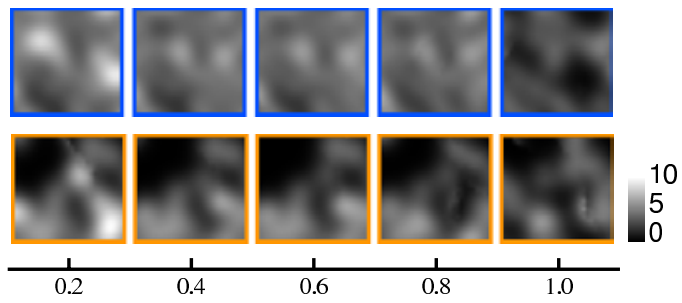

g

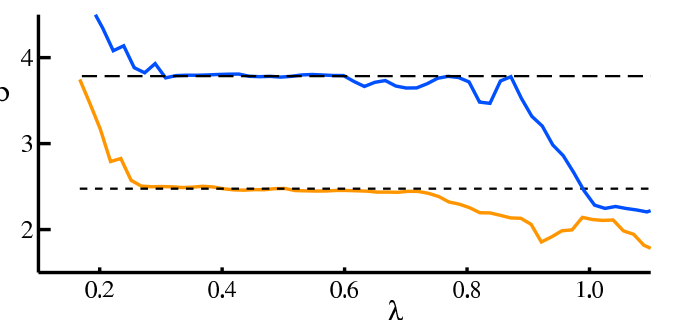

Figure 4.1: Calculating pinwheel densities. a-c, Optically recorded orientation maps in galago (a), ferret (b), and tree shrew (c) visual cortex. Each map contains a large number of pinwheels. Four pinwheels are marked by white arrows in the magnified region in $\mathbf{b}$ (black frame). $\mathbf{d}$, High and low pinwheel density regions from c magnified. e, Pinwheel locations (marked by white points) obtained by the method sketched in $\mathbf{f}$ and $\mathbf{g}$. Maps are low-pass filtered by applying a cutoff in the Fourier domain at wavelength $\lambda=0.5$ (in units of column spacing $\Lambda$; see Methods). $\mathbf{f}, \mathbf{g}$, Method. $\mathbf{f}$, Maps of local pinwheel density $\rho_{\lambda}(\mathbf{x})$ calculated from $\mathbf{d}$ after low-pass filtering with cutoff wavelengths $\lambda=\{0.2,0.4,0.6,0.8,1.0\}$. g. Spatially averaged pinwheel density $\rho_{\lambda}=\left\langle\rho_{\lambda}(\mathbf{x})\right\rangle_{\mathbf{x}}$ in each region as a function of filter strength $\lambda$. Over a broad range of filter strengths, the pinwheel density $\rho_{\lambda}$ is constant defining the pinwheel density $\rho$ in each region (orange curve, fit $\rho=2.48$ (dotted); blue curve, fit $\rho=3.78$ (dashed).

wheels only in pairs, requiring a distortion sufficiently strong to move pinwheels onto each other. In order to ensure the necessary signal accuracy, especially avoiding the effects associated with blurring [70], optical recordings were carried out using a large depth of focus (for details see e.g. [13]).

In previous studies, pinwheel densities were found to exhibit a considerable interspecies, interindividual [64, 66] and intraareal [13, 85] variability. Its largest fraction is due to the variation of column spacings $\wedge$ [44, 64]. Adapting the methods from the previous chapter for calculating the local spacing of columns (Section 4.2.3, we estimated average column spacings of $\langle\Lambda\rangle=0.69 \pm 0.02 \mathrm{~mm}$ (mean \pm s.e.m.; \langle\rangle denotes species average) for the galago, $\langle\Lambda\rangle=0.61 \pm 0.01 \mathrm{~mm}$ for the tree shrew, and $\langle\Lambda\rangle=0.88 \pm 0.01 \mathrm{~mm}$ for the ferret. Other factors of the layout being equal the pinwheel density $\hat{\rho}$ per $\mathrm{mm}^{2}$ scales as $\sim 1 / \wedge^{2}$ [92 107] implying 2.08 more pinwheels per $\mathrm{mm}^{2}$ for the ferret compared to the tree shrew. In order to eliminate all variation due to differences in column spacings $\Lambda$ and to enable a comparison among species, individuals and different regions, we defined the pin- 
wheel density by [92, 107]

$$
\rho=\hat{\rho} \wedge^{2}
$$

relative to the 'hypercolumn' of size $\Lambda^{2}$. The pinwheel density $\rho$ is dimensionless and depends only on the layout of orientation columns. The pinwheel density defined in this way is large for patchy and small for more band-like columnar organizations (e.g. Fig. 4.1d, e).

Based on the robustness of pinwheels against noise, we developed an objective and fully automated method for estimating pinwheel densities (see Section 4.2.4). To separate efficiently signal from noise in the maps, we applied a soft signal cutoff in the Fourier domain at cutoff wavelength $\lambda$. Moreover, since the structure of the noise and the spacing of columns varies typically across cortex (Fig. 4.1 $\mathrm{a}-$ c), we subdivided the maps into smaller regions (with size $\lesssim 5 \Lambda^{2}$, Fig. 4.1k-e) and analyzed the pinwheel density separately in each region. This eliminated high frequency noise without affecting the pinwheel density $\rho$, as shown in Fig. 4.1f, g. Filtering with small cutoff wavelengths preserved many spurious pinwheels in the map. Filtering with large cutoff wavelengths degraded the organization of the map. Both effects were absent within an intermediate filter regime where the filter dependent pinwheel density exhibited a plateau (Fig. $4.1 \mathrm{~g}$ ). As an operational definition, the value of the plateau is used as an estimate of the pinwheel density $\rho$ in the following. The mean pinwheel density $\rho$ of an orientation map was calculated by two independent methods (see Methods for details). In a first method, we estimated pinwheel densities $\rho$ in sufficiently small regions $\left(\lesssim 5 \Lambda^{2}\right.$, Fig. 4.1 k-e) by fitting the plateau based on visual inspection and averaged it over all regions (weighted by their area). Using a second method, we automatically fitted a function to the plateau at every location in the map (least square fit) and averaged the resulting local pinwheel density $\rho(\mathbf{x})$ over the entire map. Both methods yielded consistent results (see Section 4.2.9). Wherever not noted otherwise, presented results are based on the first method. The remainder of this section describes the two methods in more detail and presents supplementary studies.

\subsubsection{Fermi filtering}

Fermi-filtering can be used to efficiently eliminate high and low frequency noise without deteriorating the signal. Low-pass filtering can be carried out in the Fourier domain by a product

$$
\tilde{I}(\mathbf{k})=\tilde{K}(\mathbf{k}) \tilde{J}(\mathbf{k})
$$

between Fourier representations of the unfiltered map $J$ and the filter kernel $K$. Frequency components in the map $J$ at which the kernel $\tilde{K}$ is close to 0 are eliminated. Components at which the kernel is equal to 1 remain unchanged. Fig. 4.2 compares the effects of the widely used Gaussian filter kernel and the Fermi kernel (4.3). Multiplying the map with a Fermi function (blue curve) eliminates 
high frequency components while leaving the bulk of the signal unchanged. This is not, however, ensured for a Gaussian kernel (red curve). Eliminating most high frequency noise with a Gaussian kernel typically deteriorates also the bulk of the signal substantially. It is left unchanged only for weak filtering (a Gaussian with large width).

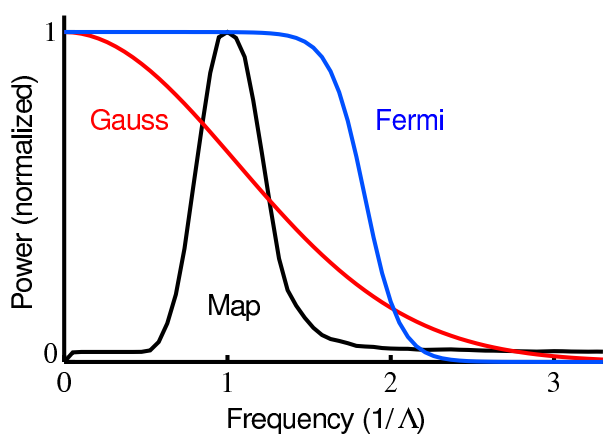

Figure 4.2: The advantage of Fermi-low-pass filtering. The black curve shows the average radial component of the power spectrum of the galago map from Fig. 1a (high-pass filtered; normalized). Superimposed are the radial components of Gaussian (red curve) and Fermi filter kernels (blue curve).

\subsubsection{Preprocessing}

A region of interest (ROI) was defined for each hemisphere containing the imaged part of area V1 (and parts of area V2 in the case of ferrets). Locations outside the ROI were excluded from further analysis. From each difference map $I^{\prime}(\mathbf{x})\left(0^{\circ}-\right.$ $\left.90^{\circ}, 45^{\circ}-135^{\circ}\right)$ we calculated a high-pass filtered map $I(\mathbf{x})=I^{\prime}(\mathbf{x})-J(\mathbf{x})$ by subtracting its local average $J(\mathbf{x})$ obtained from a product with a Fermi-function

$$
\tilde{K}_{h p}(\mathbf{k})=\frac{1}{1+e^{-\left(k_{h p}-|\mathbf{k}|\right) / \beta_{h p}}}
$$

in the Fourier domain where $k_{h p}$ is the high-pass cutoff frequency and $\beta$ controls the stiffness. To account for the boundary of the ROI, the local average $J(\mathbf{x})$ was normalized in real space by the weight $W(\mathbf{x})=\int_{R O I} K\left(\mathbf{x}^{\prime}-\mathbf{x}\right) d^{2} \mathbf{x}^{\prime}$ of the Fermi function $K$ within the ROI. We used $\beta_{h p}=0.05 k_{h p}$ and $k_{h p}=2 \pi / \lambda_{h p}$ with $\lambda_{h p}=1.6 \mathrm{~mm}$ for the ferret, $\lambda_{h p}=1.2 \mathrm{~mm}$ for the tree shrew and $\lambda_{h p}=1.4 \mathrm{~mm}$ for the galago to account for the interspecies differences of column spacing $\Lambda$. The independence of pinwheel densities from the value of the high-pass cutoff wavelength $\lambda_{h p}$ is shown for the galago as an example in Fig. 4.6.

\subsubsection{Column spacing}

Difference maps $I(\mathbf{x})$ were low-pass filtered in the Fourier domain by multiplying Eq. (4.3) with $\beta_{l p}=0.05 k_{l p}, k_{l p}=2 \pi / \lambda_{l p}$ and $\lambda_{l p}=0.3 \mathrm{~mm}$ for the ferret and $\lambda_{l p}=$ $0.2 \mathrm{~mm}$ for the galago and tree shrew. For each difference map $I$, the local spacing of columns was calculated using the wavelet method introduced previously [44 45] 
and described in more detail in Chapter 3. Morlet wavelets $\left(k_{\psi}=7, \sigma_{y}=1\right)$ were sampled with a spatial resolution of $0.05 \mathrm{~mm}$ on $n$ scales $l_{i}$ equally spaced in an interval $d$ (ferret, $n=19, d=[0.4,1.3] \mathrm{mm}$; galago and tree shrew, $n=$ $17, d=[0.3,1.1] \mathrm{mm})$ using 16 wavelet orientations $\theta_{i}=\{0 \pi / 16, \ldots, 15 \pi / 16\}$. The local column spacing $\Lambda_{I}(\mathbf{x})$ was defined by the scale $l^{*}$ maximizing the angle averaged modulus of wavelet coefficient. The maximum was interpolated using cubic splines. The local column spacing $\Lambda_{I}(\mathbf{x})$ of the two difference maps $I$ was averaged to obtain a single $2 \mathrm{D}$ map of local column spacing $\Lambda(\mathbf{x})$ for each map. In regions with artefacts in one of the maps, values were determined only from the other map. The mean column spacing was defined by the spatial average $\Lambda=$ $\langle\Lambda(\mathbf{x})\rangle_{\mathbf{x}}$ of local column spacing.

\subsubsection{Pinwheel density estimation}

We applied a variable filtering scheme to ensure filter independence of the estimated pinwheel density $\rho$. Pinwheel centers were calculated in orientation maps low-pass filtered as described above using various cutoff wavelengths $\lambda_{l p}$ (ferret, 90 values $\lambda_{l p}$ equally spaced in [0.1,0.99]mm; galago and tree shrew, 70 values in $\left.[0.1-0.79] \mathrm{mm} ; \beta_{l p}=0.005\right)$. Pinwheel centers were identified in each map by the zero contour crossings of the two difference maps [53]. The filter dependent local pinwheel density was defined as $\rho(\mathbf{x}, \lambda)=\hat{\rho}(\mathbf{x}, \lambda) \Lambda(\mathbf{x})^{2}$, where $\hat{\rho}(\mathbf{x}, \lambda)$ is the density per $\mathrm{mm}^{2}$ calculated as superposition of normalized Gaussians with $\sigma=0.25 \Lambda$ centered at the putative pinwheel locations for low-pass cutoff wavelength $\lambda_{l p}$, followed by a local rescaling $\lambda=\lambda_{l p} / \Lambda(\mathbf{x})$ expressing the cutoff wavelength relative to the local column spacing $\Lambda(\mathbf{x})$. Thus, $\rho(\mathbf{x}, \lambda)$ is the number of pinwheels in an area of size $\Lambda(\mathbf{x})^{2}$ surrounding $\mathbf{x}$ as a function of the reduced low-pass cutoff wavelength $\lambda$ (Fig. 4.1f).

Pinwheel densities $\rho$ were estimated using two different methods. The first method is based on visual inspection. We divided orientation maps into rectangular regions of size $\lesssim 5 \Lambda^{2}$ defined on a grid (Fig. 4.1k, d). For each region we calculated the filter dependent pinwheel density $\rho(\lambda)=\langle\rho(\mathbf{x}, \lambda)\rangle_{\mathbf{x}}$ by averaging over all locations $\mathbf{x}$. Typically, $\rho(\lambda)$ was constant over a broad range of cutoff wavelengths $\lambda$ (Fig. $4.1 \mathrm{~g}$ ). The pinwheel density $\rho$ of the region is given by the value of this plateau and was estimated by fitting to it a constant function $f(\lambda)=c$. The fitting was done based on visual inspection. To do this objectively, the scale on the $\rho$-axis was removed and its range shifted by an amount drawn from a centered Gaussian distribution with unit SD. Regions for which a plateau could not be identified unambiguously $(<5 \%)$ were excluded from further analysis. Pinwheel densities from different regions were averaged weighted with their size in hypercolumns $\Lambda^{2}$ to obtain the mean density $\rho$ of a map or the average density $\langle\rho\rangle$ of a number of maps. The average density $\rho$ of a map was robust against variations 
of the grid such as shifts or rotations. For cases with spatially homogeneous noise (ferret, 14 out of 82 ; galago, 7 out of 9; tree shrew, 8 out of 26) we calculated $\rho(\lambda)$ and thus $\rho$ from only one region spanning the entire map which provided very consistent estimations of $\rho$ as revealed from test cases.

The second method applies an automatic fitting scheme to estimate the pinwheel densities $\rho$. At every location $\mathbf{x}$, we fitted to the filter dependent local pinwheel density $\rho(\mathbf{x}, \lambda)$ (calculated for $\sigma=1 \Lambda$ ) in the interval $0.2<\lambda<1$ a piecewise linear function

$$
h(\lambda)=c_{0}+c_{1}\left[-\lambda+\lambda_{0}\right]_{+}+c_{2}\left[\lambda-\left(\lambda_{0}+\Delta \lambda\right)\right]_{+}
$$

that exhibits a plateau of value $c_{0}$ ranging from $\lambda_{0}$ to $\lambda_{0}+\Delta \lambda$ (least-squares fit; []$_{+}$denotes rectification). The slopes $c_{1}$ and $c_{2}$ accounted for the $\lambda$-dependence of $\rho(\mathbf{x}, \lambda)$ outside of the plateau (compare Fig. $4.1 \mathrm{~g}$ ). Across cortical locations and different maps, plateaus differed in their position and extent. To ensure an optimal sampling of a plateau, we varied its position $\lambda_{0}$ and extension $\Delta \lambda$ in $h$ (constraint by $\lambda_{0} \geq 0.2, \Delta \lambda \geq 0.4$, and $\left.0.2+\lambda_{0}+\Delta \lambda \leq 1\right)$, and then defined the local pinwheel density $\rho(\mathbf{x})$ by $c_{0}$ from the best fit. Averaging over space yielded the pinwheel density $\rho$ of a given area.

\subsubsection{Pinwheel locations}

The location of pinwheels was defined using a cutoff wavelength $\lambda=\xi$ at the center of the plateau of $\rho(\lambda)$ (Fig. 4.1 ). This method can introduce artificial pinwheel 'copies' close to real pinwheels if $\xi$ varies over space. These pinwheels were identified based on their topological charge and eliminated. While accurately identifying the density of pinwheels, pinwheel locations were only determined approximately. Since pinwheels are topologically stable, slight filter dependent shifts are consistent with the same total number.

\subsubsection{Nearest neighbor distances}

From the pinwheel locations we calculated the distances $h$ of a pinwheel to its nearest neighbors. Since pinwheels are characterized by a topological charge, three distances are of particular interest: the distance $h_{++,--}$to the nearest pinwheel of equal sign, the distance $h_{+-,-+}$to the nearest pinwheel of opposite sign, and $h_{t o t}$, the smallest distance to any other pinwheel. The distance $h_{t o t}$ is always the smaller of the two other distances. We determined these distances for each pinwheel at location $\mathbf{x}_{0}$, say, and normalized them by the local column spacing $\Lambda\left(\mathbf{x}_{0}\right)$ at the location of the considered pinwheel. Distributions include distances from all pinwheels in a set of maps. 


\subsubsection{Systematic variation}

The map of systematic variation of local pinwheel density $\rho_{\text {syst }}(\mathbf{x})$ was calculated by superimposing and averaging all maps of local pinwheel density $\rho(\mathbf{x})$ from a species. Maps from different hemispheres were aligned along their V1/V2 border and their estimated representation of the horizontal meridian. To achieve this, maps from right hemispheres were mirror inverted. Locations were included where $\geq 10$ hemispheres contributed to the calculation of $\rho_{\text {syst }}(\mathbf{x})$.

\subsubsection{Permutation tests}

Significances were tested by comparison with randomly permuted data. The $p$ value was the fraction of pseudo values larger than the real one. The distance of the average pinwheel density $\langle\rho\rangle$ in real and random maps and those of the mean SD $\langle s\rangle$ in different species were compared to pseudo distances obtained by randomly permuting the data among both groups. The SD of the systematic variation $\rho_{\text {syst }}(\mathbf{x})$ of the tree shrew was compared to pseudo SDs obtained from superpositions of equally many $(\mathrm{N}=26)$ maps randomly drawn from all ferret hemispheres $(\mathrm{N}=82)$.

\subsubsection{Comparison of fully and semi automatic method}

The two methods for estimating pinwheel densities $\rho$ provided very consistent results. This is demonstrated in Fig. 4.3 comparing the pinwheel densities $\rho$ obtained by the two methods. For each species, values were highly correlated with $r=0.91$ for tree shrews, $r=0.93$ for galagos and $r=0.82$ for ferrets. For individual maps, pinwheel densities calculated by the two methods differed on average by $2.4 \%$ for the tree shrew, $2.7 \%$ for the galago, and $4.3 \%$ for the ferret.

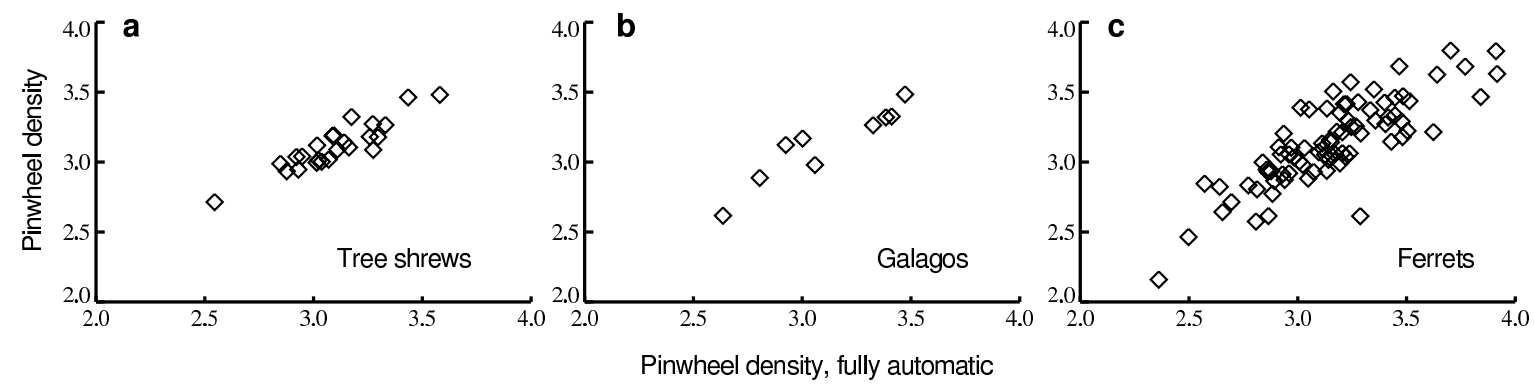

Figure 4.3: Comparison of methods. a-c, Scatter plots of pinwheel densities $\rho$ calculated by the fully- and semi automatic method. Pinwheel densities were highly correlated (tree shrew, $r=0.91$, galago; $r=0.93$; ferrets, $r=0.82$ ).

The starting point for both methods is the filter dependent pinwheel density $\rho(\mathbf{x}, \lambda)$. Using the semi-automatic method the density $\rho(\mathbf{x}, \lambda)$ is first averaged over 
regions $\lesssim 5 \Lambda^{2}$ defined by an arbitrary arranged regular grid superimposed on the map. Then the pinwheel density $\rho$ of the region is estimated by visual inspection from the plateau in the averaged filter dependent density $\rho(\lambda)$. Using the fully automatic method, the pinwheel density is estimated from the plateau of $\rho(\mathbf{x}, \lambda)$ by an automatic fitting procedure before averaging the result over space. The advantage of the semi automatic method is that the estimation of the plateau can be checked for at every step. In some regions the plateau is more irregular: Values may fluctuate around an average value, plateaus may be relatively short, or there may be multiple plateaus at different ranges of cutoff wavelength $\lambda$. In these cases, a thorough inspection appears necessary. Regions for which a plateau cannot be determined unambiguously (regions with poor signal to noise ratio) can be identified by this inspection and excluded from further analysis.

\subsubsection{Measurement of column spacing by Fourier analysis}

To estimate column spacings locally we used wavelets. The mean column spacing $\Lambda$ of a given map can be calculated from local column spacing $\Lambda(\mathbf{x})$ by averaging over space. An alternative method for calculating the column spacing $\Lambda$ is provided by Fourier analysis. From each difference map $I(\mathbf{x})$ we calculated its two dimensional power spectrum

$$
\mathcal{P}(\mathbf{k})=|\tilde{I}(\mathbf{k})|^{2}
$$

as the squared modulus of the Fourier representation of the map $I(\mathbf{x})$. Averaging over the angle,

$$
P(k)=\int_{0}^{2 \pi} \frac{d \theta}{2 \pi} \frac{1}{k} \mathcal{P}(k, \theta),
$$

yielded the one dimensional spectrum $P$. The dominant frequency $q$ of the spectrum $P$ determines the typical spacing through $\Lambda=2 \pi / q$. To estimate the frequency $q$ we smoothed the spectrum $P$ with a Gaussian kernel of width $\sigma_{k}=2 \pi / \sigma_{x}$ with $\sigma_{x}=1 \mathrm{~mm}$ and fitted it with the function

$$
G(k)=a_{0} \exp \left(-\frac{\left(k-a_{1}\right)^{2}}{2 a_{2}^{2}}\right)+a_{3}+a_{4} k+a_{5} k^{2}
$$

where the $a_{i}$ are fitt parameters and $q=a_{1}$ (nonlinear least-squares fit).

Fig. 4.4 shows scatter plots of mean column spacings $\wedge$ for the three analyzed species calculated by the wavelet and by the Fourier method. Values were very similar in all species. In tree shrews, the correlation was $r=0.99$. Relative distances of column spacings $\Lambda$ obtained by the two methods were on average $1.5 \%$, 

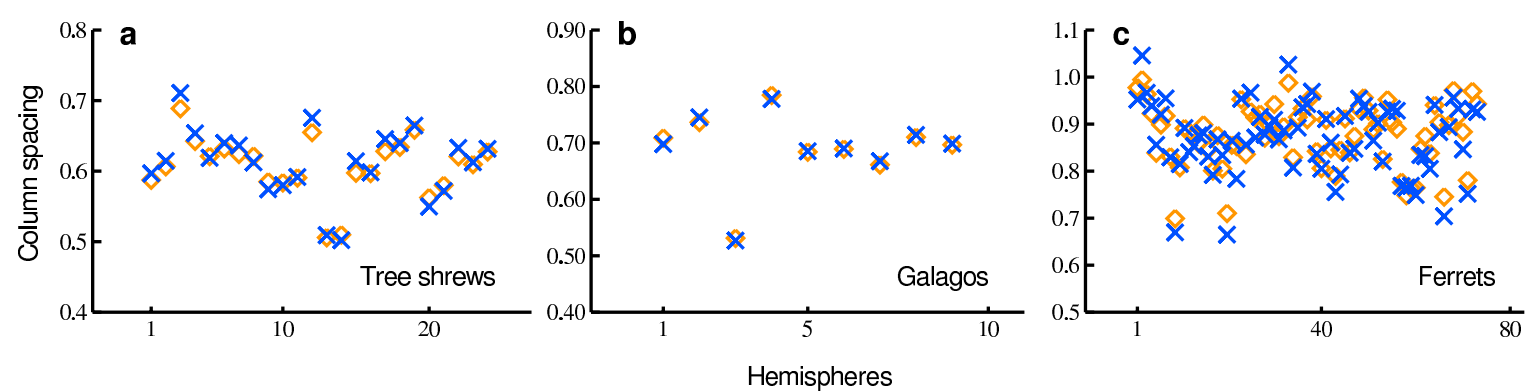

Figure 4.4: Consistency of column spacing estimation. a-c, Column spacings $\Lambda$ calculated by wavelet analysis (blue crosses) and by Fourier analysis (orange diamonds). Column spacings were highly similar (average distance (relative): tree shrew, 1.5\%; galago 0.7\%; ferret, $2.3 \%$ ).

and the distance of the average column spacing $\langle\Lambda\rangle$ was $0.7 \%$. In galagos, the correlation was $r=0.99$ with average distances $0.7 \%$ and distance of averages $0.01 \%$. In ferrets, we found $r=0.95,2.3 \%$ and $0.7 \%$, respectively. Deviations between the Fourier and wavelet method were mainly due to a variation of column spacings across cortex. While generally small in the galago, in tree shrews this variation could exceed $>10 \%$ in some of the cases, and in ferrets (see the examples in Fig. 4.1). Since these variations are better captured by the nonlocal wavelet analysis, the values obtained with this method appear more appropriate. Nevertheless, the deviations between the two methods may be taken as an estimate for the error of column spacing measurement. Concerning the average pinwheel density $\langle\rho\rangle$, these errors imply a possible absolute error of $\approx 0.04$ in tree shrews, $\approx 0.001$ in galagos, and $\approx 0.05$ in ferrets. We conclude that Fourier analysis confirms the estimation of column spacings by wavelet analysis and thus of pinwheel densities.

\subsubsection{Age-dependence}

Pinwheel densities do not depend on age, as indicated by $\mathrm{N}=82$ ferret cases recorded between postnatal day (PD) 30 and $>270$. In the ferret, orientation maps are not observed before PD 30 [17]. As shown in Fig. 4.5. most of the data from ferret visual cortex was recorded within this period. However, a clear dependence of pinwheel densities on age was not indicated. It may be absent or weak but covered by the substantial variation of values.

\subsubsection{Dependence of pinwheel density on high-pass filtering}

Low frequency noise has only a weak effect on pinwheel densities. The origin of low frequency signal components in optical data is mainly the curvature of the cortex implying various sources of inhomogeneity such as variation in light reflectance, light absorption and cortical depth of recording. Whereas high frequency 


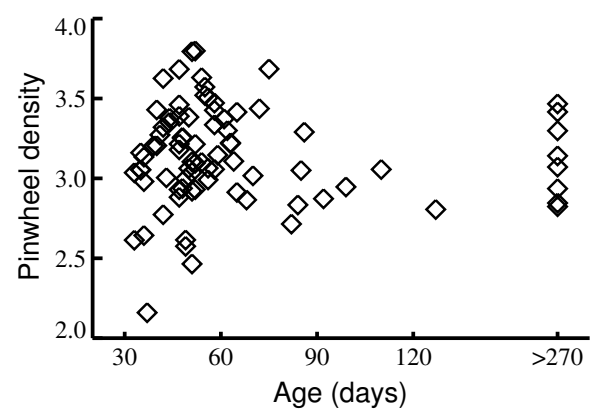

Figure 4.5: Pinwheel densities are independent of age. Pinwheel densities $\rho$ of maps from $\mathrm{N}=82$ ferret hemispheres vs. age at recording time. The abscissa is shortened between day 120 and day 270 .

noise can induce a large number of spurious pinwheels, low frequency noise is less problematic. In addition, low frequency noise is often dominant on spatial scales very distinct from the scale of the signal and therefore easily separable.

We tested the impact of low frequency noise on the pinwheel density by varying the high-pass cutoff wavelength $\lambda_{h p}$ underlying the analysis. As a representative example we analyzed galagos. Fig. 4.6 shows the average pinwheel density $\langle\rho\rangle$ of the galago for various cutoff wavelengths $\lambda_{h p}$. For each value of $\lambda_{h p}$ the entire analysis was performed including the calculation of the map of local column spacings $\Lambda(\mathbf{x})$ and the filter dependent pinwheel density $\rho(\mathbf{x}, \lambda)$. The fully automatic method was used to estimate pinwheel densities $\rho$. Apart from the high-pass cutoff wavelength $\lambda_{h p}$, all parameters were identical for each data point. We found that the average pinwheel density $\langle\rho\rangle$ was nearly constant between $\lambda_{h p}=1.0$ and $\lambda_{h p}=2.0$ demonstrating the robustness of the pinwheel density estimation against low frequency noise.

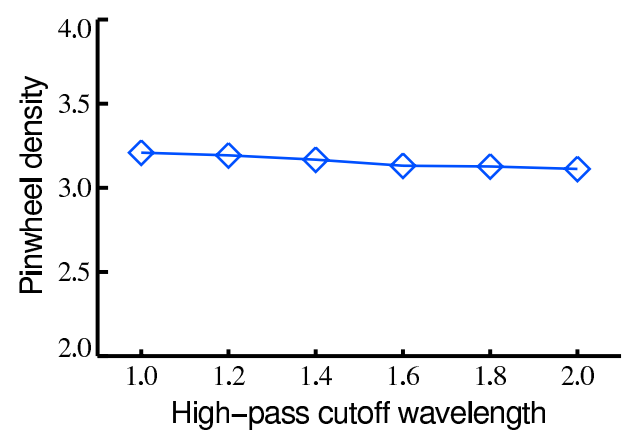

Figure 4.6: Dependence on high-pass filtering. Average pinwheel density $\langle\rho\rangle$ for various highpass cutoff wavelength $\lambda_{h p}$. Values are $\langle\rho\rangle=$ $3.21,3.19,3.17,3.13,3.13,3.11$ (from left to right).

\subsection{Universal pinwheel density in galago, ferret and tree shrew}

We found that the average pinwheel density $\langle\rho\rangle$ was, up to the precision provided by our analysis, indistinguishable in the three analyzed species. In 26 tree shrew hemispheres, the average pinwheel density was $\langle\rho\rangle=3.12 \pm 0.04$ (mean \pm s.e.m.; 

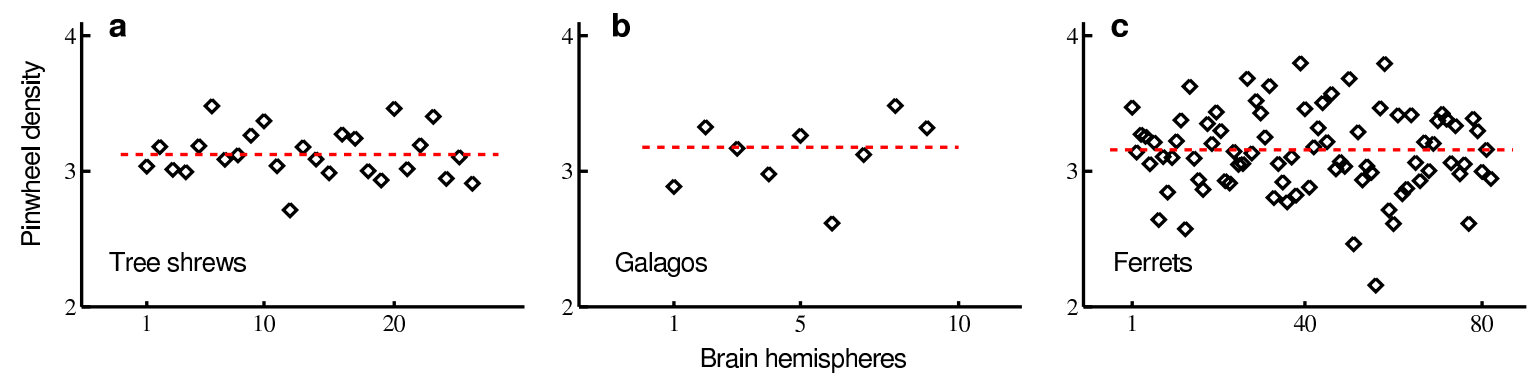

Figure 4.7: Universal pinwheel density in the mammalian visual cortex. a-c, Pinwheel densities $\rho$ in tree shrews (a, $N=26$ hemispheres), galagos $(\mathbf{b}, N=9)$, and ferrets $(\mathbf{f}, \mathrm{N}=82)$. Red dashed lines in a-c represent average pinwheel densities $\langle\rho\rangle$ (tree shrew, $3.12 \pm 0.04$; galago, $3.18 \pm 0.09$; ferret, $3.16 \pm 0.03$; mean \pm s.e.m., weighted by the size of maps in units of $\Lambda^{2}$, see Methods).

weighted by the size of the maps) with mean pinwheel densities $\rho$ in individual maps ranging from 2.7 to 3.5 (Fig. 4.7/). In 9 galago hemispheres, pinwheel densities $\rho$ varied comparably with an average of $\langle\rho\rangle=3.18 \pm 0.09$ (Fig. 4.7p). An average pinwheel density of $\langle\rho\rangle=3.16 \pm 0.03$ was found in 82 ferret hemispheres (Fig. 4.7k). Mean pinwheel densities $\rho$ varied stronger across individual ferret maps, between 2.0 and 4.0. The average pinwheel density in all three species was $\langle\rho\rangle=3.14 \pm 0.03$. These results were confirmed by the fully automatic method with average pinwheel densities $\langle\rho\rangle=3.11 \pm 0.04$ for the tree shrew, $\langle\rho\rangle=3.17 \pm 0.10$ for the galago, $\langle\rho\rangle=3.17 \pm 0.03$ for the ferret, and $\langle\rho\rangle=3.14 \pm 0.03$ for the total average.

\subsubsection{Uncertainty of average pinwheel density estimation}

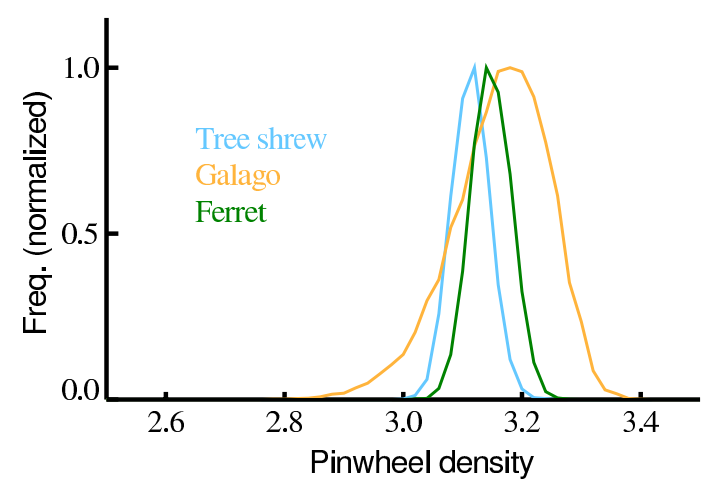

The range of possible values of average pinwheel density that is consistent with the data set can be estimated using the method of boot strapping. The histograms of values for the three species are shown in Fig. 4.8. The width of distributions
Figure 4.8: The range of average pinwheel densities. Averages were calculated by boot strapping from the data from Fig. 2 ( $\mathrm{N}=10000$ samples for each species). SD of histograms: tree shrew, 0.03; galago, 0.08 ; ferret, 0.03 . 
measured by their SDs were 0.03 for tree shrew, 0.08 for the ferret and 0.03 for the galago. These values agreed well with the estimation of the s.e.m. in Fig. 4.7 . Thus, possible averages ranged between 3.06 and 3.18 for the tree shrew, between 3.10 and 3.22 for the ferret, and between 3.02 and 3.34 for the galago ( $\pm 2 S D$ from mean).

\subsubsection{Random maps}
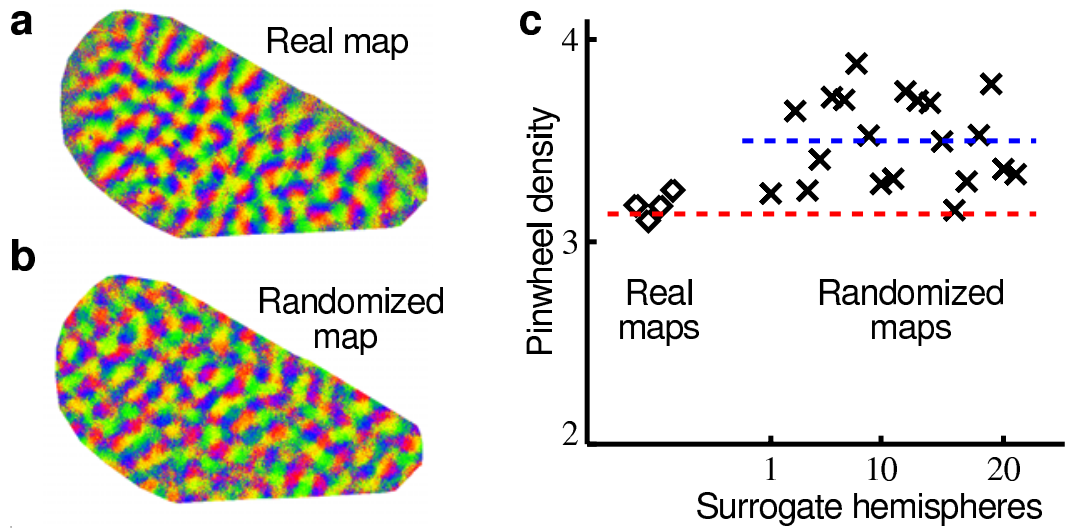

Figure 4.9: Pinwheel densities in real and random orientation maps. a, Tree shrew orientation map from Fig. 1c. b, Random map generated from a by phase shuffling in the frequency domain. c. Pinwheel densities $\rho$ of random maps (crosses, $N=20$ ) and of original maps (diamonds, $\mathrm{N}=4$ [tree shrew, $\mathrm{N}=2$; galago, $\mathrm{N}=2$ ]; 5 random maps were generated from each original map). The blue dashed line represents the average pinwheel density $\langle\rho\rangle$ of random maps ( $3.50 \pm 0.05)$, the red dashed line the average in the three species $(3.14 \pm 0.03)$. Note that average densities are significantly different $(p<0.0001$, permutation test).

The observed pinwheel densities indicate that in the three analyzed species the average pinwheel density is tightly selected. In principle, orientation maps can be constructed exhibiting any pinwheel density [70 107]. How likely is it to obtain similar average pinwheel densities by chance in maps comparable to experimental maps? To answer this question, we investigated random orientation maps with identical second order statistics (Fig $4.9 \mathrm{a}, \mathrm{b}$ ). Random maps were generated by transforming maps from the tree shrew and galago into the Fourier domain, randomizing all phases, and transforming it back into real space, restricting the new map to a geometry identical to the measured maps. By this procedure, an arbitrary large ensemble of surrogate maps with identical spatial correlation functions can be generated for each measured map. From each of 4 different orientation maps ( 2 from galago and 2 tree shrew) we created 5 surrogate maps resulting in a total of $\mathrm{N}=20$ random orientation maps. We found that pinwheel densities $\rho$ 
ranged between 3.15 and 3.9 (Fig. $4.9 k$ ). The average density $\langle\rho\rangle=3.50 \pm 0.05$ $(\langle\rho\rangle=3.54 \pm 0.04$ for the automated scheme) was considerably larger than for the real maps $\left(p<10^{-4}\right.$; random tree shrew maps only, $p<10^{-3}$; galago random maps only, $p<10^{-4}$; permutation test). Thus, pinwheel densities observed in orientation maps in the visual cortex are highly exceptional in comparable random maps suggesting the action of a nontrivial selection principle.

\subsection{Local irregularity and universal pinwheel statistics}

\subsubsection{Large scale selection}
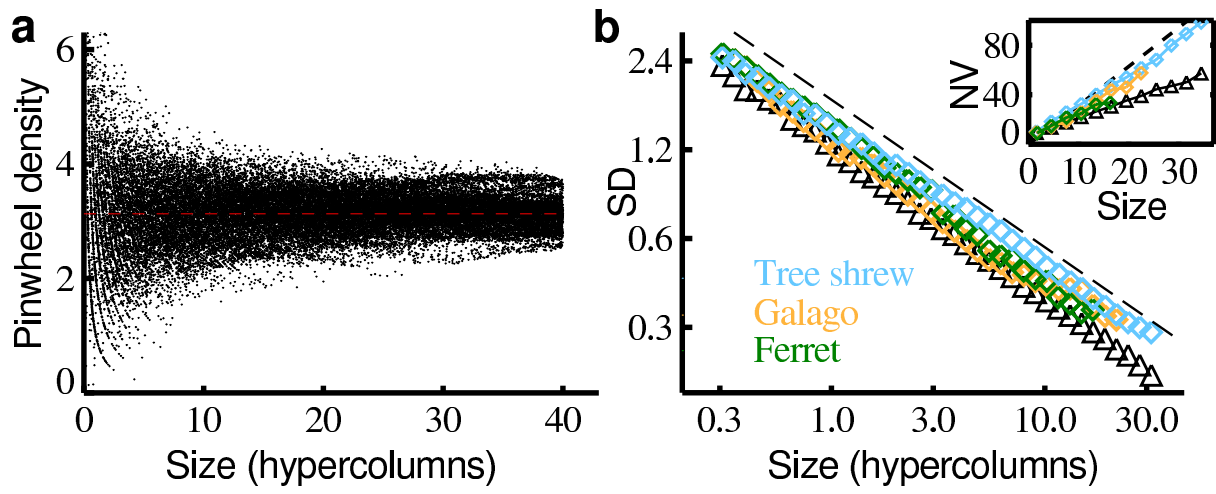

Figure 4.10: Large scale selection of pinwheel density. a, Pinwheel densities $\rho$ in regions of various size $A$ randomly selected from tree shrew orientation maps $\left(4 \times 10^{4}\right.$ circular regions (partially truncated by map boundaries) with sizes uniformly distributed in $0<A<30 \wedge^{2}$ drawn from $\mathrm{N}=26$ tree shrew maps with sizes $>30 \wedge^{2}$ ). The red dashed line marks the average tree shrew pinwheel density $\langle\rho\rangle=3.12$. $\mathbf{b}$, SDs of pinwheel densities $\rho$ in randomly selected regions for the three species (diamonds) and for the random maps from Fig. 3 (black triangles, density corrected). The black dashed curve represents the SD for a 2D Poisson process of equal density. The inset shows the number variance (NV) increasing as $c A\langle\rho\rangle$ with $c \approx 0.9$ for the tree shrew, $c \approx 0.8$ for ferret and galago, $c=1$ for Poisson and $c \approx 0.5$ for random maps.

Are pinwheel densities also selected locally or is there a minimum cortical region to which such a selection principle applies? To answer this question we randomly drew subregions of various size $A$ from orientation maps and calculated their pinwheel densities $\rho$. Fig. 4.10a shows the distribution of densities $\rho$ for the tree shrew as a representative example. In small regions $A<5 \wedge^{2}$, pinwheel densities $\rho$ varied substantially with smallest values $\rho \approx 1$ and largest values $\rho>5$. In regions of size $A \approx 15 \Lambda^{2}$ values covered a smaller range between 2 and 4 . The variation was similar to those found for the ferret maps (Fig. 4.7k) exhibiting an average size of $\approx 15 \Lambda^{2}$. For larger regions, pinwheel densities $\rho$ converged towards the 
species mean $\langle\rho\rangle$. In all three species, the variability (SD) of pinwheel densities decreased proportional to $\sqrt{A}$ such that the number variance $(\mathrm{NV})$ increased linearly as $c A\langle\rho\rangle$. The proportionality factor was $c \approx 0.9$ for the tree shrew and $c \approx 0.8$ for ferret and galago (Fig. 4.10p). The NV was smaller than for a 2D Poisson process with $c=1$, but larger compared to the phase shuffled maps from Fig. 4.9 for which we found $c \approx 0.5$.

By extrapolating the decay of SD to the size $A_{V 1}$ of the entire V1, we estimated the true variation of pinwheel densities $\rho$ among individual hemispheres. We obtained a SD of $\approx 0.08$ for galagos assuming that V1 covers $A_{V 1} \approx 200 \mathrm{~mm}^{2}$ [73] $\approx 420 \wedge^{2}$ and, likewise, a SD of $\approx 0.16$ for tree shrews and ferrets where $A_{V 1} \approx 100 \wedge^{2}$ [48]. Thus, whereas the average density of pinwheels appears to be tightly selected even within an individual brain hemisphere, the local density varies substantially and in a similar fashion in all three species.

\subsubsection{Systematic inhomogeneity}

Not all properties of orientation maps are universal. Indeed, distinct map designs are consistent with the observed pinwheel statistics. For instance, the tree shrew differs from the other species by its often stripe-like and thus pinwheel sparse organization of orientation columns along the V1/V2 border region and at the caudal pole. Fig. 4.11a exemplifies this by showing the map of local pinwheel density $\rho(\mathbf{x})$ for the tree shrew orientation map from Fig. 4.1k (see Methods). Local densities $\rho(\mathbf{x})$ varied from below 1 at the V1/V2 border to values larger than 4 in more central representations. Densities varied comparably in tree shrew and ferret maps $(p=0.28$, permutation test, Fig. 4.11p) and exhibited indistinguishable distributions across all maps (Fig. 4.11k). However, in the tree shrew, pinwheel-sparse and pinwheel-rich regions covaried in maps from different individuals, whereas in the ferret, such regions were less related in different animals. This is revealed by the maps of systematic variation of local pinwheel density $\rho_{\text {syst }}(\mathbf{x})$ for the tree shrew (Fig. $4.11 \mathrm{~d}$ ) and the ferret (Fig. 4.11 $)$ ) calculated by superimposing and averaging maps of local pinwheel density $\rho(\mathbf{x})$ from different animals. In the tree shrew, pinwheel densities were systematically lower than average along the V1/V2 border and at the caudal pole $\left(\rho_{\text {syst }}(\mathbf{x})<2.5\right)$, and, compensating for this, higher than average in the representation of near central eccentricities $\left(\rho_{\text {syst }}(\mathbf{x})>3.5\right)$. The histogram of $\rho_{\text {syst }}(\mathbf{x})$ was significantly wider for the tree shrew (Fig. 4.11F, b, $p=0.005$, permutation test). In contrast, distributions of nearest neighbor distances of pinwheels were very similar in the two species (Fig. $4.11 \mathrm{~g}, \mathrm{~h}$ ) and also in the galago (data not shown). The histograms of these distances are statistics sensitive to the local arrangement of pinwheels indicating that despite the different systematic organization, local map properties are remarkably similar in the two species. 


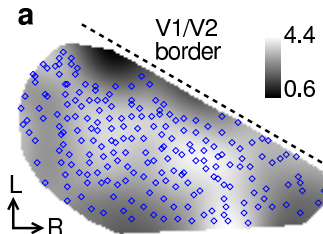

d

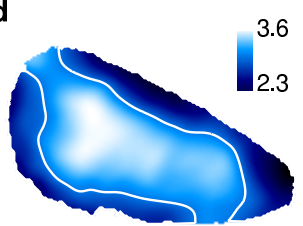

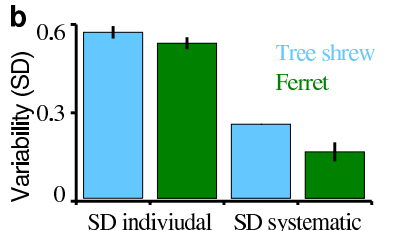

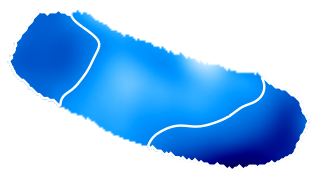

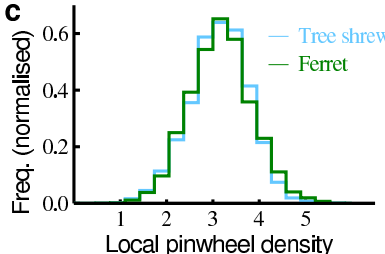

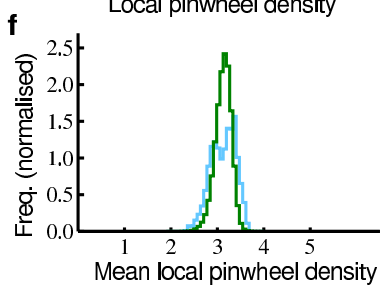

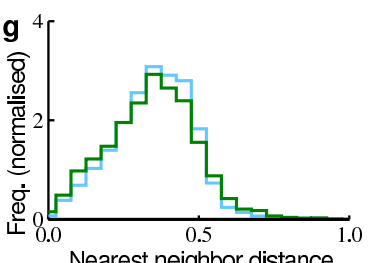

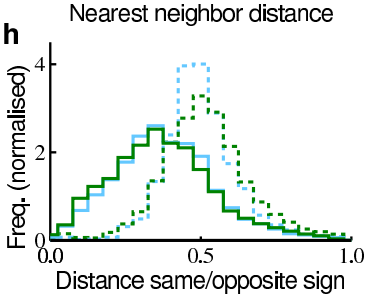

Figure 4.11: Species specific properties of pinwheel densities. a, Map of local pinwheel density $\rho(\mathbf{x})$ (grey scale coded, see Methods) with pinwheel locations superimposed (blue diamonds) for the orientation map from Fig. 4.1p (mean pinwheel density, $\rho=3.08$; SD, 0.63). b. SDs of local pinwheel density $\rho(\mathbf{x})$ (left; mean over hemispheres; black bars indicate s.e.m.) and of systematic variation of local pinwheel density $\rho_{\text {syst }}(\mathbf{x})$ (right; from $\mathrm{N}=26$ hemispheres; ferret, mean and s.e.m. calculated from $10^{4}$ subsets $(\mathrm{N}=26)$ randomly selected out of the $\mathrm{N}=82$ hemispheres). c, Histograms of local pinwheel densities $\rho(\mathbf{x})$ from all hemispheres. $\mathbf{d}$, e, Maps of systematic variation of local pinwheel density $\rho_{\text {syst }}(\mathbf{x})=\langle\rho(\mathbf{x})\rangle$ for the tree shrew (d) and ferret (e) calculated by superimposing and averaging maps $\rho(\mathbf{x})$ from all hemispheres at corresponding cortical locations (see Methods; blue scale coded; contour line drawn at average). $\mathbf{f}$, Histograms of $\rho_{\text {syst }}(\mathbf{x})$ (ferret, average histogram for subsets from $\mathbf{b}$, right). $\mathbf{g}, \mathbf{h}$, Distributions of nearest neighbor distances $h_{\text {tot }}$ between pinwheels of arbitrary topological charge $(\mathbf{g})$, and of distances $h_{++,--}$between pinwheels of equal (dashed) and $h_{+-,-+}$of opposite charge (solid) (h).

\subsection{Conclusion}

We found that several properties of visual cortical orientation maps are universal in the ferret, tree shrew and galago. Relative to the typical distance of columns which is specific for a given species, pinwheels occurred with a constant average density. Furthermore, in the three species, pinwheels exhibited an equal degree of spatial variation and a very similar statistics of nearest neighbor distances. This appears in contrast to the substantial differences reported for other columnar systems such as ocular dominance which is organized into regular blobs in the galago [110], in domains of rather irregular shape and size in the ferret [102], and in laminae in the tree shrew [42]. The universal organization of orientation columns is reminiscent of many examples of complex systems where structures within a particular universality class are selected by self-organization despite differences in details of the microscopic dynamics [23]. In the following chapter, we therefore analyze the pinwheel statistics in solutions of a model formalizing the idea of self-organization 
of orientation columns in the visual cortex. 


\title{
5 Theory of pinwheel density selection
}

\author{
Eyes are round.
}

(Claudia Benze)

\subsection{Motivation}

What mechanism can explain the observed universality of pinwheel statistics in the visual cortex? A common hypothesis states that random forces might form the structure of orientation maps. However, this hypothesis is hard to reconcile with our observation that pinwheel densities cannot be reproduced by random maps with second order statistics identical to real maps. Also genetic factors seem unlikely taking into account that the investigated species are separated for more than 30 million years of evolution. And sensory instruction, if prevailing, would rather counteract a universal organization given the substantial differences in their upstream visual systems in the different animals. An alternative explanation is offered by self-organization of cortical circuitry. Self-organization has been observed to robustly produce large scale structures in various complex systems. Often, the class of pattern emerging depends on fundamental system properties such as symmetries rather than on the system specific details. Its formation can therefore often be described by an effective model incorporating only these properties. The robust occurrence of a universal pinwheel statistics suggests that pinwheel formation may be explained by a simple model neglecting many details of cortical connectivity. In analogy to the theory of complex systems we therefore adopted a generalized Swift-Hohenberg model of the form Eq. (2.5) with nonlinearities derived from key features of visual cortical organization. The model includes the effects of longrange intracortical connections between columns of similar orientation preference.

We study the average pinwheel density, the count statistics of pinwheels, and their nearest neighbor distributions for solutions of the generalized Swift-Hohenberg model (2.5) close to the bifurcation point. In the limit of infinite interaction range, we calculate the average pinwheel density analytically. The results are checked by numerical simulations away from the bifurcation point. We find that for a realistic range of nonlocal interaction, the model reproduces the observed pinwheel statistics quantitatively. 


\subsection{Methods}

\subsubsection{The model}

The model is defined by the integro-differential equation

$$
\partial_{t} z(\mathbf{x}, t)=L z(\mathbf{x}, t)-N[z(\mathbf{x}, t)]
$$

with a Swift-Hohenberg type [89] linear part

$$
L=r-\left(q_{c}^{2}+\nabla^{2}\right)^{2}
$$

where $r$ is the bifurcation parameter governing the stability of the homogeneous solution. For $r<0$, the homogeneous solution $z(\mathbf{x})=0$ is stable. For $r>0$, a pattern starts growing via a Turing instability. Near criticality, the critical wavenumber $q_{c}=\frac{2 \pi}{\Lambda}$ defines the column spacing $\Lambda$ of the pattern. The nonlinear part is given by

$$
N=(g-1)|z|^{2} z+(2-g)\left(z K_{\sigma} *|z|^{2}+\frac{1}{2} \bar{z} K_{\sigma} * z^{2}\right)
$$

where $*$ denotes convolution, $K_{\sigma}$ is a Gaussian

$$
K_{\sigma}=\frac{1}{2 \pi \sigma^{2}} e^{-\frac{x^{2}}{2 \sigma^{2}}}
$$

and $0 \leq g \leq 2$ controls the relative strength of local and non-local interactions. The model minimizes an energy functional during development. For a more detailed description of this model, refer to Chapter 2.

\subsubsection{Numerical integration}

To numerically obtain solutions of Eq. (5.1), the use of spectral methods is advantageous particularly when solutions satisfying periodic boundary conditions are sought. Moreover, both the spatial derivatives of the linear part (5.2) and the computationally demanding convolutions of the nonlinear part can be treated efficiently with spectral methods. Spatial derivatives become multiplications in Fourier representation avoiding phase errors commonly introduced when calculating derivatives with finite difference methods. To solve Eq. (5.1) numerically in a rectangular region, the field $z(x, y, t)$ was sampled on a regular grid

$$
\begin{aligned}
& x_{i}=\frac{(i-1) L_{x}}{N_{x}}, \quad i=1, \ldots N_{x} \\
& y_{j}=\frac{(j-1) L_{y}}{N_{y}}, \quad i=1, \ldots N_{y}
\end{aligned}
$$


with $L_{x}$ and $L_{y}$ the dimensions of the region and $N_{x}$ and $N_{y}$ the number of sample points in the $x$ and $y$ directions. The amplitudes in the frequency domain $\tilde{z}\left(\left(q_{x}\right)_{k},\left(q_{y}\right)_{l}, t\right)$ are related to the sampled field $z\left(x_{i}, y_{j}, t\right)$ through the discrete Fourier transform

$$
\tilde{z}=\sum_{i=1}^{N_{x}} \sum_{j=1}^{N_{y}} z\left(x_{i}, y_{j}, t\right) e^{-i\left(q_{x}\right)_{k} x_{i}} e^{-i\left(q_{y}\right){ }_{1} y_{j}}
$$

where the wave numbers are confined to the set $\left(q_{x}\right)_{k}=\frac{2 \pi k}{L_{x}}$ with $-\frac{N_{x}}{2}+1 \leq k \leq$ $\frac{N_{x}}{2}$ and to $\left(q_{y}\right)_{l}=\frac{2 \pi l}{L_{y}}$ with $-\frac{N_{y}}{2}+1 \leq l \leq \frac{N_{y}}{2}$, respectively. By the backward transform

$$
z=\frac{1}{N_{x} N_{y}} \sum_{k=-N_{x} / 2-1}^{N_{x} / 2} \sum_{l=-N_{y} / 2-1}^{N_{y} / 2} \tilde{z}\left(\left(q_{x}\right)_{i},\left(q_{y}\right)_{i}, t\right) e^{i\left(q_{x}\right)_{k} x_{i}} e^{i\left(q_{y}\right) y_{j}}
$$

the field $z$ can be recovered from the modes $z$. In Fourier representation, the linear part (5.2) is diagonal with eigenvalues

$$
\lambda\left(q_{x}, q_{y}\right)=r-\left(q_{c}^{2}-\left(q_{x}^{2}+q_{y}^{2}\right)\right)^{2},
$$

that are positive around the critical wavenumber $q_{c}=\frac{2 \pi}{\Lambda}$ only. The Fourier transform 5.6 translates Eq. (5.1) into a system of nonlinear ordinary differential equations

$$
\dot{\tilde{z}}=\lambda \tilde{z}-\tilde{N}(\tilde{z}, t)
$$

in which the coupling among the components of $\tilde{z}$ is mediated through the nonlinearity

$$
\tilde{N}(\tilde{z}, t)=(g-1) \tilde{z} * \tilde{z} * \tilde{z}+(2-g)\left(\tilde{z} *\left(\tilde{K}_{\sigma} \tilde{z} * \tilde{z}\right)+\frac{1}{2} \tilde{z} *\left(\tilde{K}_{\sigma} \tilde{z} * \tilde{z}\right)\right),
$$

the Fourier representation of the nonlinear part (5.3). The nonlinear term (5.10) was evaluated by pseudospectral methods where convolutions in real space are calculated by products in Fourier space while convolutions in Fourier space are calculated by products in real space, respectively. For a finite collection of amplitudes this method leads to aliasing of modes which can cause numerical instabilities. However, the strong linear damping by the biharmonic term suppresses contaminated modes provided there is sufficient resolution in real space. For a cubic nonlinearity, modes with wavenumbers $q_{\text {alias }} \geq \frac{q_{\max }}{2}=\frac{n \pi}{2 L}$ are subject to aliasing. Therefore, the maximal wavenumber $q_{\max }$ must be chosen large enough to ensure $q_{\max }>2\left(q_{c}+\delta / 2\right)$ where $\delta$ is the width of the range of wavenumbers around $q_{c}$ not efficiently suppressed by the linear part (5.8). Numerical tests revealed that 
for $r \leq 0.3$ a ratio of $\frac{q_{c}}{q_{\max }} \leq \frac{2}{5}$ provided sufficient suppression of all effects due to aliasing.

Because of the stiffness of the biharmonic operator contained in the linear part (5.8), an implicit temporal integration scheme was necessary, since an explicit timemarching procedure would require the use of an unacceptably small time step [71]. To achieve this, the linear term was integrated using an exponential propagation procedure. The nonlinear term was treated explicitly by a predictor corrector scheme, interpreting Eq. (5.9) formally as a system of linear equations modified by a nonlinear forcing term (following [24]). Multiplying both sides of the system 5.9 by the integrating factor $\exp (-\lambda t)$ leads to

$$
\left.e^{-\lambda t^{\prime}} \tilde{z}\left(t^{\prime}\right)\right|_{t} ^{t+\Delta t}=\int_{t}^{t+\Delta} d t^{\prime} \tilde{N}\left(\tilde{z}\left(t^{\prime}\right), t^{\prime}\right) e^{-\lambda t^{\prime}}
$$

after integration over one time step $\Delta t$. Assuming a sufficiently smooth solution $\tilde{z}$ the nonlinear term $\tilde{N}$ can be approximated in the time interval $t \leq t^{\prime} \leq t+\Delta t$ by a linear function

$$
\tilde{N}\left(\tilde{z}\left(t^{\prime}\right), t^{\prime}\right) \approx \tilde{N}_{0}+\left(t^{\prime}-t\right) \tilde{N}_{1}
$$

where

$$
\begin{aligned}
& \tilde{N}_{0}=\tilde{N}(\tilde{z}(t), t), \\
& \tilde{N}_{1}=\frac{\tilde{N}(\tilde{z}(t+\Delta t), t+\Delta t)-\tilde{N}(\tilde{z}(t), t)}{\Delta t}
\end{aligned}
$$

are determined from the solution $\tilde{z}$ at the endpoints of the time interval. Inserting the linear approximation (5.12) with (5.13) into (5.11) one obtains

$$
e^{-\lambda(t+\Delta t)} \tilde{z}(t+\Delta t)=e^{-\lambda t} \tilde{z}(t)+\tilde{N}_{0} \int_{t}^{t+\Delta t} d t^{\prime} e^{-\lambda t^{\prime}}+\tilde{N}_{1} \int_{t}^{t+\Delta t} d t^{\prime}\left(t^{\prime}-t\right) e^{-\lambda t^{\prime}} .
$$

After performing the integrals this results into the time marching scheme

$$
\tilde{z}(t+\Delta t)=e^{\lambda \Delta t} \tilde{z}(t)+\tilde{N}_{0} \frac{e^{\lambda \Delta t}-1}{\lambda}+\tilde{N}_{1} \frac{e^{\lambda \Delta t}-(1+\lambda \Delta t)}{\lambda^{2}}
$$

for advancing $z$ from $t$ to $t+\Delta t$. Note that expression (5.15) is still implicit since the term $\tilde{N}_{1}$ depends also on $\tilde{z}(t+\Delta t)$. To evaluate $\tilde{z}(t+\Delta t)$, Eq. (5.15) was solved iteratively by a predictor-corrector procedure. Setting in a first step $N_{1}=0$, the obtained solution $\tilde{z}(t+\Delta t)$ was used to estimate $\tilde{N}_{1}$, and to revaluate $\tilde{z}(t+\Delta t)$ with this guess in a second step. This two step procedure already provided sufficient accuracy for our demands (see below). It can be shown that this temporal integration scheme is accurate to 2 nd order [24]. 
To ensure accuracy of the solution, its numerical error was controlled by means of an adaptive step size procedure [71] adjusting the time step $\Delta t$ after every step in order to maintain a predefined accuracy $\epsilon$. After each step the distance

$$
\Delta \tilde{z}=\left|\Delta \tilde{z}_{\Delta t}-\Delta \tilde{z}_{\Delta t / 2}\right|
$$

between solutions $\tilde{z}(t+\Delta t)$ obtained for step size $\Delta t$ and $\frac{\Delta t}{2}$ was calculated. If the maximal fractional error defined by

$$
\Delta=\frac{\max \{\Delta \tilde{z}\}}{\max \left\{\left|\tilde{z}_{\Delta t / 2}\right|\right\}}
$$

exceeded the desired accuracy $\epsilon$ the step was reevaluated using the rescaled step size

$$
\Delta^{\prime} t=c \Delta t\left(\frac{\epsilon}{\Delta}\right)^{1 / 3}
$$

where the exponent accounts for the scaling of the error $\Delta \sim \Delta t^{3}$ in the used integration scheme and $c$ is a safety factor. To ensure a maximal possible step size during integration, a scaling inverse to (5.18) was applied to the subsequent step whenever the error $\Delta$ was below the desired accuracy $\epsilon$. For all simulations $\epsilon=10^{2}$ and $c=0.9$ was used.

Solutions were obtained on a $128 \times 128$ mesh using aspect ratios of $\Gamma=17-25$ ( $\Gamma=D / \wedge$ where $D$ is the diameter of the system). Initial conditions $z_{0}=z(t=0)$ were band-pass filtered Gaussian random fields with spectral width $\delta k=k_{c}$ and average amplitude $\mathcal{A}=r$. Computer runtime increased approximately linear with the time $t$ rescaled by the intrinsic time constant $T=1 / r$ of the linear part (5.2). The speed of marching was $\approx 1 \times 10^{3} / 180 \mathrm{~s}=1 \times 10^{6} / 50 \mathrm{~h}$ on a Pentium $4(2 \mathrm{GHz})$. Consistency of the results was tested by single runs using a larger mesh-size of $256 \times 256$.

\subsubsection{Linear wavelength selection}

The purpose of this Section is to illustrate the behavior of the model during the initial phase of the dynamics.

A solution of Eq. (5.1) starting from a band-pass filtered Gaussian random map is shown in Fig. 5.1. The time $t$ has been rescaled by the time constant

$$
T=\frac{1}{r}
$$

associated with the growth rate of the linear part (5.2). The rescaling Eq. (5.19) of time will be applied throughout this chapter. While, initially, the map varied on 
a
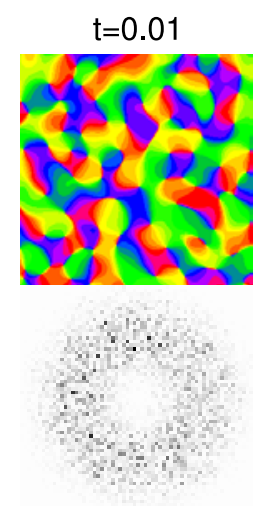

b

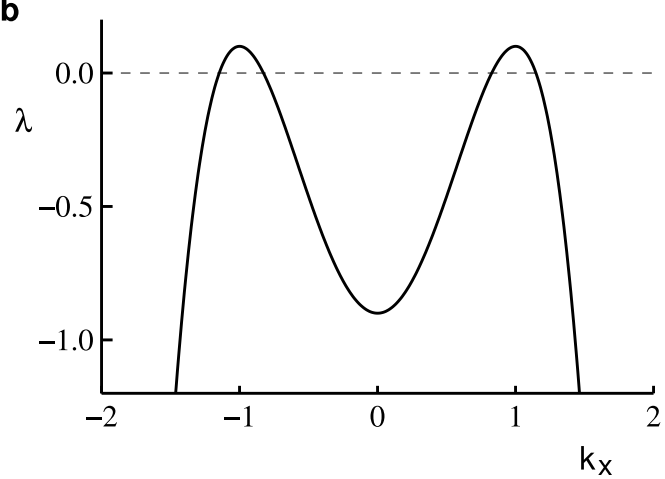

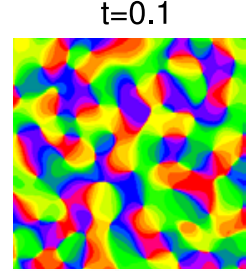

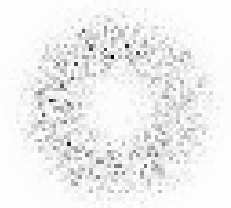

$t=1$

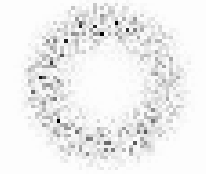

$t=10$
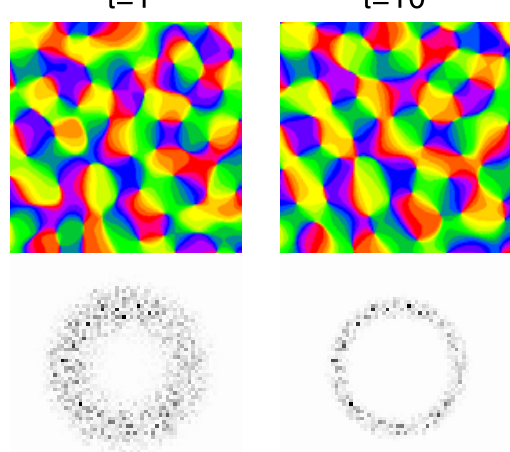

$t=100$

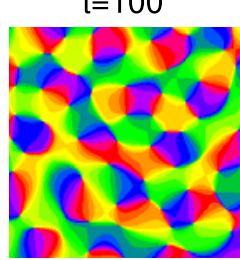

C

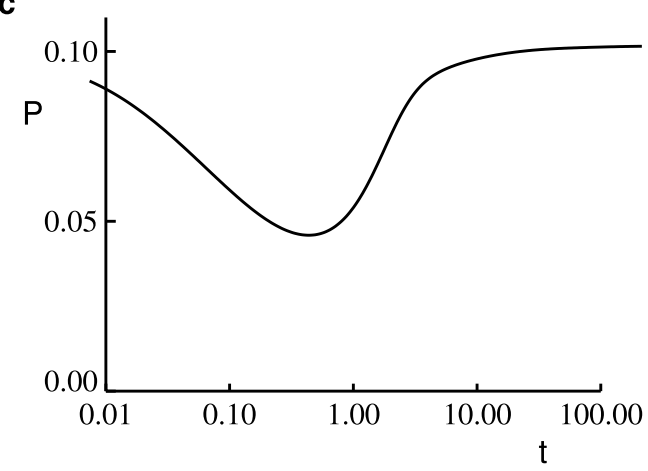

Figure 5.1: Numerical solution of Eq. (5.1) using integration scheme (5.15). a, Early development of angle map (upper row, subregion of size $A=(4.25 \Lambda)^{2}$, smoothed for illustration) and power spectrum (lower row, origin of Fourier-space in center of annulus, only central region covering the critical circle shown). Starting from a band-pass filtered Gaussian random map at $t=0$, the solution was advanced to $t=100$ (rescaled by $T=1 / r$ ) using periodic boundary conditions and parameters $g=0.98, r=0.1, \sigma=1.7, \Gamma=17$ on a mesh size $128 \times 128$. b, Cross section through the spectrum $\lambda$ of the linear part of Eq. 5.1. Modes $\exp \left(i k_{x} x\right)$ with $k_{x} \simeq q_{c}=1$ have positive growth rate. c, Power $P$ (Eq. (5.20) $)$ of the evolving solution.

various spatial scales reflected by a broad power spectrum, shortly after its organization became more ordered and regularly spaced with energy mostly confined to the selected wavelength $\Lambda=\frac{2 \pi}{k_{c}}$ (Fig. 5.11). This behavior is manly due to the action of the linear part (5.2) forcing modes on the critical circle $|\mathbf{k}|=k_{c}$ to grow with rate $r$ while strongly suppressing those further away (Fig. 5.1p). Following Eq. (5.8), this growth is uncoupled. Acting as a band-pass filter, the structure related to scale $\Lambda$ remained unchanged whereas larger and smaller scales were eliminated. Fig. 5.1k shows the time course of the total power of the field

$$
P(t)=\left\langle|z(\mathbf{x}, t)|^{2}\right\rangle_{\mathbf{x}}
$$


where \langle\rangle$_{\mathbf{x}}$ denotes average over space, reflects the different growth rates among modes. The power $P$ initially distributed over various modes with $P(0)=r=0.1$ first decreased until $t \approx 1$ due to the suppression of modes outside the critical circle, but later on increased again until saturating on a level of $\approx r$ after $t \approx 10$. At this time, the growth of amplitudes on the critical circle saturated with an approximate modulus

$$
|A| \approx \sqrt{\frac{r}{1+g(n-1)}}
$$

predicted by weakly nonlinear analysis, where $n$ is the number of active modes in a planform solution (Chapter 2). Eq. (5.21) implies a stationary power of

$$
P \approx \frac{n r}{1+g(n-1)}
$$

consistent with Fig. 5.1 where $g=0.98$ has been used. At the time the amplitudes of the modes reach near stationary strengths, the influence of the nonlinear part becomes comparable to the linear part in Eq. (5.1) both scaling as $\sim r^{3 / 2}$. At this stage, at $t \equiv t_{o} \approx 10^{1}$, the phase of nonlinear competition between modes begins that leads to reorganization of the structure of the map and ultimately to pattern selection. In parts, it can be analyzed by means of the amplitude formalism (Chapter 2), but the richness of the dynamics will only get apparent in numerical solutions of the full dynamics.

\subsubsection{Pinwheel identification}

Pinwheel centers were identified by the crossings of the zero contour lines of the real and imaginary part of the field $z$. To calculate the expectation value of the pinwheel density $\langle\rho\rangle$ in an ensemble of planforms of order $n$ (Section 5.3), planforms 5.23) with randomly chosen sets of wavevector directions $l_{j}$ and phases $\phi_{j}$ were synthesized with aspect ratio $\Gamma=32$ on a $2048 \times 2048$ grid. In each planform, pinwheel locations were identified within a quadratic subregion of size $8 \wedge \times 8 \wedge$. Realizations were collected until sufficient precision of average pinwheel densities $\langle\rho\rangle$ was reached, measured by the standard error measure (SEM) $\Delta=s / \sqrt{N}$, were $s$ is the standard deviation (SD) of densities $\rho$ and $N$ the number realizations. The afforded precision of $\Delta<0.03$ for $5 \leq n \leq 14$ and $\Delta<0.01$ for $15 \leq n \leq 20$ required between 100 and 1000 realizations per order $n$.

To analyze pinwheels in numerical solutions of Eq. (5.1) (Section 5.5), solutions $\tilde{z}(\mathbf{k}, t)$ to Eq. 5.9) were synthesized on a $512 \times 512$ grid providing sufficient spatial resolution of the field $z(\mathbf{x}, t)$ in real space. Pinwheels were identified in each map in a quadratic subregions of $3 / 4$ of the total width spanning an area of $12 \Lambda \times 12 \Lambda-$ $21 \wedge \times 21 \wedge$ dependent on the aspect ratio $\Gamma$. 


\subsection{Pinwheel densities near criticality}

Solutions of model Eq. (5.1) close to the bifurcation point $r=0$ are of particular interest. In this regime, the relevant solutions are known in close form, derived by means of the amplitude formalism (see Chapter 2), allowing for a precise estimation of the expectation value of quantities of interest such as the pinwheel density. Furthermore, since the form of the amplitude equations is universal near the bifurcation point for a large class of models, conclusions derived in this regime remain valid also for variations of model (5.1). The solutions obtained near the bifurcation point at $r \ll 1$ serve as a baseline for investigations further away from criticality at $r>0$ which can at present only be carried out by numerical methods. While dependencies on the details of the model are expected to play a role for $r>0$, solutions in this regime can still be categorized by those obtained for $r \ll 1$.

As outlined in Chapter 2 for $r \ll 1$ solutions of Eq. (5.1) are planforms

$$
z(\mathbf{x})=\sqrt{\frac{2}{n}} \sum_{j=0}^{n-1} e^{i\left(l_{j} \mathbf{k}_{j} \mathbf{x}+\phi_{j}\right)}
$$

of order $n$ consisting of $n$ active modes with wavevectors

$$
\mathbf{k}_{j}=k_{c}\left(\cos \left(\frac{j \pi}{n}\right), \sin \left(\frac{j \pi}{n}\right)\right)
$$

distributed equally on the upper half of the critical circle $|\mathbf{k}|=k_{c}$, with binary variables $l_{j} \in\{-1,1\}$ determining whether the mode with wave vector $\mathbf{k}_{j}$ or $-\mathbf{k}_{j}$ is active, and with arbitrary phases $\phi_{j}$. The order $n$ of the selected solution depends on the two model parameters $\sigma$ and $g$ and scales for given $g$ approximately linear as

$$
n \simeq 2 \pi \sigma
$$

For a given $n$, the family of solutions consists of all possible combinations of directions $l_{j}$ and phases $\phi_{j}$ and has a degenerated potential.

\subsubsection{Pinwheel densities for intermediate interaction range}

The specific interaction range $\sigma$ for a given species or animal depends on the range of intracortical long-range horizontal connections (see Fig. 2.2). Estimated values are approximately in the interval $0.5 \Lambda \lesssim \sigma \lesssim 3 \Lambda$. It is therefore important to analyze the pinwheel density in planforms of intermediate order of range $3 \lesssim n \lesssim$ 20 given that $n$ and $\sigma$ are related through Eq. (5.25). To proceed in this, planforms of various order $n$ were synthesized numerically with randomly chosen $l_{j}$ and $\phi_{j}$ (see Methods). The pinwheel density $\rho$ was calculated in each realization by identifying all pinwheel locations within a pre-defined subregion by the zero crossings of the 
a

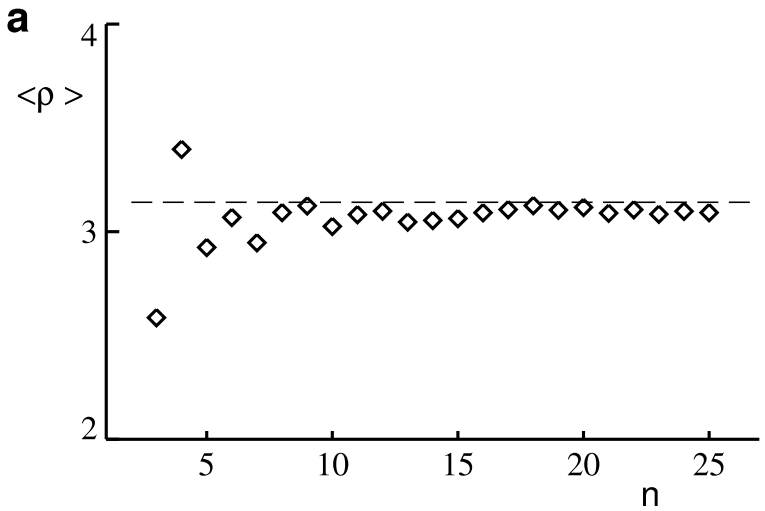

b

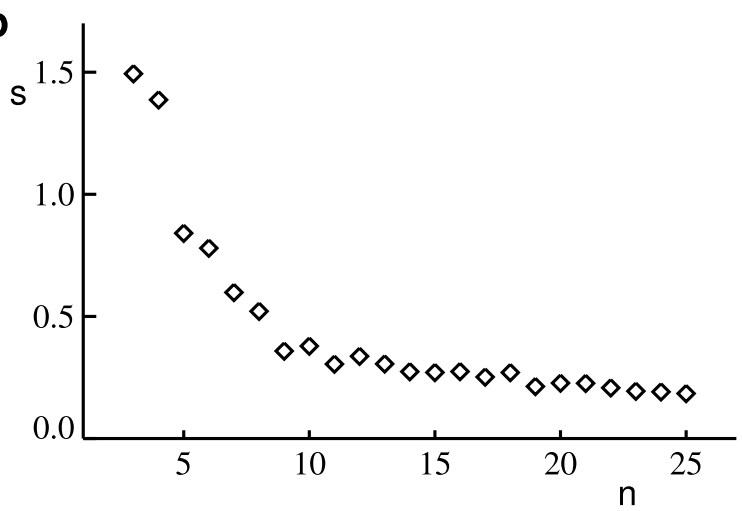

Figure 5.2: Pinwheel densities in model Eq. (5.1) near the bifurcation point $(r \ll 1)$. a, Average pinwheel density $\langle\rho\rangle$ in planforms of order $n$ (diamonds). Planforms with random sets of wavevector directions $l_{j}$ and phases $\phi_{j}$ were synthesized on $2048 \times 2048$ grids with aspect ratio $\Gamma=32$. For each planform the pinwheel density was determined in a quadratic $\Gamma=8$ subregion. Planforms were drawn until a achieving SEM $\Delta=0.03$ for $n<$ 15 and $\Delta=0.01$ for $n \geq 15$. The dashed line represents $\langle\rho\rangle=\pi$, valid in the limit $n \rightarrow \infty$. Note that the average pinwheel density $\langle\rho\rangle$ is close to $\pi$ even at intermediate orders of $n$. $\mathbf{b}$, Standard deviation (SD) $s$ of densities $\rho$. Note that $s$ decreases with order $n$.

real and imaginary part of (5.23). From the ensemble of realizations of order $n$ the average pinwheel density $\langle\rho\rangle$ and the SD $s$ were determined. Using this method, with a sufficient large number of realizations, both quantities can be evaluated with arbitrary precision.

Fig. $5.2 \mathrm{a}$ shows the average pinwheel density $\langle\rho\rangle$ of planforms of various orders ranging between $3 \leq n \leq 25$. Among smaller orders $n \leq 7$, average densities $\langle\rho\rangle$ were found to fluctuate substantially covering the range $2.5 \leq\langle\rho\rangle \leq 3.5$. However, they were much more confined between $2.9 \leq\langle\rho\rangle \leq 3.2$ for intermediate orders $8 \leq n \leq 15$. For large order $n>15$, the ensemble average $\langle\rho\rangle$ appeared to converge towards $\pi$ from below. Furthermore, as shown in Fig. $5.2 \mathrm{p}$, the variation $s$ of pinwheel densities $\rho$ in different realizations decreased drastically with $n$. For large $n$, it became successively smaller indicating that for large $n$ both the average pinwheel density $\langle\rho\rangle$ and the pinwheel density $\rho$ of a single realization are close to $\pi$.

\subsubsection{Limit of large interaction range: Planform anisotropy}

These results highlighted in Fig. 5.2 suggest that in the limit $n \rightarrow \infty$, the equality $\langle\rho\rangle=\pi$ is exact. To show this, we first calculate the average pinwheel density $\left\langle\rho_{l}\right\rangle$ for an ensemble of planforms (5.23) with a fixed set of wavevector directions 
$l=\left(l_{0}, l_{1}, \ldots, l_{n-1}\right)$ but arbitrary phases $\phi_{j}$ in the limit $n \rightarrow \infty$. Here, and in the following \langle\rangle denotes average over all phases $\phi_{j}$. In this limit, local linear functionals of $z$ have Gaussian statistics such that the density of pinwheels is determined by the second order statistics of the field. In a second step, the average over all sets of $l$ is evaluated as the expectation value of densities $\left\langle\rho_{l}\right\rangle$.

Pinwheels are the zeros of the field (5.23). The number of pinwheels in a given area $A$ is obtained by

$$
N=\int_{A} d^{2} \mathbf{x} \delta(z(\mathbf{x})) J(z(\mathbf{x})),
$$

where

$$
J(z(\mathbf{x}))=\left|\frac{\partial R(\mathbf{x})}{\partial x} \frac{\partial I(\mathbf{x})}{\partial y}-\frac{\partial R(\mathbf{x})}{\partial y} \frac{\partial I(\mathbf{x})}{\partial x}\right|
$$

is the Jacobian of the field

$$
z(\mathbf{x})=R(\mathbf{x})+i I(\mathbf{x})
$$

split for convenience into its real and imaginary part. Averaging Eq. (5.26) over the ensemble of phases $\phi_{j}$ reads

$$
\langle N\rangle=\int_{A} d^{2} \mathbf{x}\left\langle\delta(z(\mathbf{x}))\left|\frac{\partial(R(\mathbf{x}), I(\mathbf{x}))}{\partial(x, y)}\right|\right\rangle
$$

implying that

$$
\left\langle\rho_{l}\right\rangle=\left\langle\delta(z(\mathbf{x}))\left|\frac{\partial(R(\mathbf{x}), I(\mathbf{x}))}{d(x, y)}\right|\right\rangle
$$

is the expectation value of the pinwheel density for a fixed set of $l$.

The expectation value (5.30) only depends on local quantities, namely on the field (5.23) and its spatial derivatives

$$
\nabla z(\mathbf{x})=i \sqrt{\frac{2}{n}} \sum_{j=0}^{n-1} l_{j} \mathbf{k}_{\mathbf{j}} e^{i\left(l_{j} \mathbf{k}_{j} \mathbf{x}+\phi_{j}\right)}
$$

such that owing to the central limit theorem, both quantities have Gaussian statistics in the large $n$ limit. Eq. (5.30) is therefore determined by the first and second order statistics of $z$ and $\nabla z$. Furthermore, since their statistics is the same at each location $\mathbf{x}$, it is sufficient to evaluate Eq. (5.30) for $z(\mathbf{0}), \nabla z(\mathbf{0})$. The spatial dependency can be omitted in the following. Thus, the average in Eq. (5.30) is given by an integral over the joint probability density

$$
p(v)=\frac{1}{(2 \pi)^{3} \sqrt{\operatorname{det} C}} e^{-\frac{1}{2} v^{T} C^{-1} v}
$$


of components

$$
v=\left(R, I, \partial_{x} R, \partial_{x} I, \partial_{y} R, \partial_{y} I\right)
$$

with covariance matrix $C$ which shall be analyzed in the following.

First, the diagonal elements of $C$ are evaluated. Using (5.23) and (5.28) the autocorrelations of the field are

$$
\begin{aligned}
\left\langle R^{2}\right\rangle & =\frac{2}{n} \sum_{j, j^{\prime}=0}^{n-1}\left\langle\cos \phi_{j} \cos \phi_{j^{\prime}}\right\rangle \\
& =1
\end{aligned}
$$

for the real part and

$$
\begin{aligned}
\left\langle I^{2}\right\rangle & =\frac{2}{n} \sum_{j, j^{\prime}=0}^{n-1}\left\langle\sin \phi_{j} \sin \phi_{j^{\prime}}\right\rangle \\
& =1
\end{aligned}
$$

for the imaginary part. For the spatial derivatives one obtains

$$
\begin{aligned}
\left\langle\left(\partial_{x} R\right)^{2}\right\rangle & =\frac{2}{n} \sum_{j, j^{\prime}=0}^{n-1} l_{j} l_{j^{\prime}} k_{x j} k_{x j^{\prime}}\left\langle\sin \phi_{j} \sin \phi_{j^{\prime}}\right\rangle \\
& =\frac{1}{n} \sum_{j=0}^{n-1} k_{x j}^{2}
\end{aligned}
$$

where $k_{x j}$ is the $x$-component of $\mathbf{k}_{j}$ and, likewise,

$$
\begin{aligned}
\left\langle\left(\partial_{x} I\right)^{2}\right\rangle & =\frac{1}{n} \sum_{j} k_{x j}^{2} \\
\left\langle\left(\partial_{y} R\right)^{2}\right\rangle & =\frac{1}{n} \sum_{j} k_{y j}^{2} \\
\left\langle\left(\partial_{y} I\right)^{2}\right\rangle & =\frac{1}{n} \sum_{j} k_{y j}^{2}
\end{aligned}
$$

The equality of these correlations follows from inserting Eq. (5.24) into (5.36) yield- 
ing

$$
\begin{aligned}
\left\langle\left(\partial_{x} R\right)^{2}\right\rangle & =\frac{k_{c}^{2}}{n} \sum_{j=0}^{n-1} \cos ^{2}\left(\frac{j \pi}{n}\right) \\
& =\frac{k_{c}^{2}}{n} \sum_{j=0}^{n-1} \sin ^{2}\left(\frac{j \pi}{n}-\frac{\pi}{2}\right) \\
& =\frac{k_{c}^{2}}{n} \sum_{j=0}^{n-1} \sin ^{2}\left(\frac{j \pi}{n}\right) \\
& =\left\langle\left(\partial_{y} R\right)^{2}\right\rangle
\end{aligned}
$$

and also

$$
\begin{aligned}
\left\langle\left(\partial_{x} R\right)^{2}\right\rangle & =\frac{k_{c}^{2}}{n} \sum_{j=0}^{n-1} \cos ^{2}\left(\frac{j \pi}{n}\right) \\
& =k_{c}^{2}-\frac{k_{c}^{2}}{n} \sum_{j=0}^{n-1} \sin ^{2}\left(\frac{j \pi}{n}\right) \\
& =k_{c}^{2}-\left\langle\left(\partial_{x} R\right)^{2}\right\rangle
\end{aligned}
$$

such that all auto-correlations become

$$
\left\langle\left(\partial_{x} R\right)^{2}\right\rangle=\left\langle\left(\partial_{y} R\right)^{2}\right\rangle=\left\langle\left(\partial_{x} I\right)^{2}\right\rangle=\left\langle\left(\partial_{y} I\right)^{2}\right\rangle=\frac{k_{c}^{2}}{2}=2 \pi^{2}
$$

when choosing without loss of generality the column spacing to be $\Lambda=2 \pi / k_{c} \equiv 1$.

Most off-diagonal elements of the covariance matrix $C$ vanish. All non-vanishing contributions are related to the planform anisotropy $\xi$ depending on the set $l$. The covariance between the field and its derivative reads

$$
\begin{aligned}
\langle z \nabla \bar{z}\rangle & =-\frac{2}{n} i \sum_{j, j^{\prime}=0}^{n-1} l_{j^{\prime}} \mathbf{k}_{j^{\prime}}\left\langle e^{i\left(\phi_{j}-\phi_{j^{\prime}}\right)}\right\rangle \\
& =-\frac{2}{n} i \sum_{j=0}^{n-1} l_{j} \mathbf{k}_{j} \\
& =-2 i \vec{\chi}
\end{aligned}
$$

with $\vec{\chi} \equiv 1 \sum_{j=0}^{n-1} l_{j} \mathbf{k}_{j} \geq 0$. The modulus $\chi=|\vec{\chi}|$ is small for an isotropic distribution of wavevectors $l_{j} \mathbf{k}_{j}$. To estimate its upper bound $\chi_{\max }$, consider the most anisotropic case with all $l_{j} \mathbf{k}_{j}$ situated in the right plane $\left(l_{j}=1\right.$ for $j \leq n / 2, l_{j}=-1$ for $j>n / 2$ ). For large $n$ this upper bound is 


$$
\begin{aligned}
\chi_{\max } & =\left|\frac{1}{n} \sum_{j=0}^{n-1} l_{j} \mathbf{k}_{j}\right| \\
& =\frac{k_{c}}{\pi}\left|\sum_{j=-\frac{n}{2}}^{\frac{n}{2}-1} \frac{\pi}{n} e^{i \pi \frac{j}{n}}\right| \\
& \approx \frac{k_{c}}{\pi}\left|\int_{-\frac{\pi}{2}}^{\frac{\pi}{2}} d \alpha e^{i \alpha}\right| \\
& =4
\end{aligned}
$$

such that the anisotropy defined by

$$
\vec{\xi} \equiv \frac{1}{4 n} \sum_{j=0}^{n-1} l_{j} \mathbf{k}_{j}
$$

has its modulus $\xi=|\vec{\xi}|$ within $0 \leq \xi \leq 1$. In the following, without loss of generality, it is assumed $\vec{\xi}=\xi(1,0)$ implying that all correlations involving one derivative in $y$-direction vanish. Correlations involving $\partial_{x}$ are obtained by writing Eq. (5.41) and

$$
\begin{aligned}
\langle z \nabla z\rangle & =\frac{2}{n} i \sum_{j, j^{\prime}=0}^{n-1} l_{j^{\prime}} k_{j^{\prime}}\left\langle e^{i\left(\phi_{j}+\phi_{j^{\prime}}\right)}\right\rangle \\
& =0
\end{aligned}
$$

in the form

$$
\begin{aligned}
& \left\langle z \nabla_{x} \bar{z}\right\rangle=\left\langle R \partial_{x} R\right\rangle+\left\langle I \partial_{x} I\right\rangle+i\left(\left\langle I \partial_{x} R\right\rangle-\left\langle R \partial_{x} I\right\rangle\right)=-i 8 \xi \\
& \left\langle z \nabla_{x} z\right\rangle=\left\langle R \partial_{x} R\right\rangle-\left\langle I \partial_{x} I\right\rangle+i\left(\left\langle I \partial_{x} R\right\rangle+\left\langle R \partial_{x} I\right\rangle\right)=0
\end{aligned}
$$

and comparing both imaginary parts showing that

$$
-\left\langle I \partial_{x} R\right\rangle=\left\langle R \partial_{x} I\right\rangle=4 \xi
$$

does not vanish for anisotropic planforms.

Expression (5.46) are the only non-vanishing non-diagonal elements of the matrix C. Indeed,

$$
\left\langle R \partial_{x} R\right\rangle=\left\langle I \partial_{x} I\right\rangle=0,
$$


follows from comparing both real parts in Eq. (5.45). Furthermore, correlations between the real and imaginary part and between their derivatives, e.g.

$$
\begin{aligned}
\langle R I\rangle & =0 \\
\left\langle\partial_{x} R \partial_{x} I\right\rangle & =0 \\
\left\langle\partial_{y} R \partial_{x} I\right\rangle & =0,
\end{aligned}
$$

vanish since they contain terms of the form $\left\langle\sin \phi_{j} \cos \phi_{j^{\prime}}\right\rangle=\left\langle\sin \phi_{j}\right\rangle\left\langle\cos \phi_{j}\right\rangle=$ 0 . Finally, because

$$
\begin{aligned}
\left\langle\partial_{x} R \partial_{y} R\right\rangle & =\frac{2}{n} \sum_{j j^{\prime}} l_{j} l_{j^{\prime}} k_{j x} k_{j^{\prime} y}\left\langle\sin \phi_{j} \sin \phi_{j^{\prime}}\right\rangle \\
& =\frac{1}{n} \sum_{j} k_{j x} k_{j y} \\
& =\frac{2 k_{c}^{2}}{n} \sum_{j=0}^{n-1} \sin \left(2 \pi \frac{j}{n}\right)
\end{aligned}
$$

vanishes for arbitrary $n$, also correlations between derivatives in different directions,

$$
\left\langle\partial_{x} R \partial_{y} R\right\rangle=\left\langle\partial_{x} I \partial_{y} I\right\rangle=0,
$$

do not contribute to the density of pinwheels.

Altogether, the covariance matrix for the vector $v=\left(R, I, \partial_{x} R, \partial_{x} I, \partial_{y} R, \partial_{y} I\right)$ reads

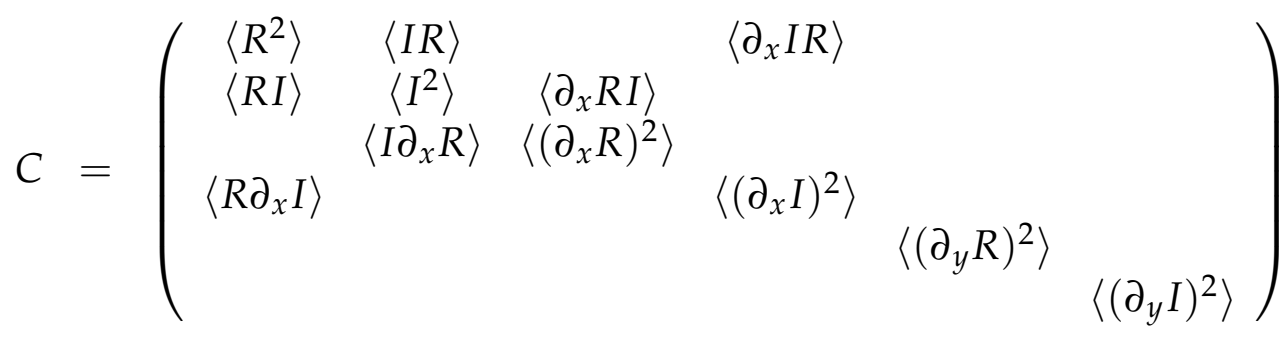

$$
\begin{aligned}
& =\left(\begin{array}{cccccc}
1 & \multicolumn{5}{c}{4 \xi_{l}} \\
& 1 & -4 \xi_{l} & & & \\
& -4 \xi_{l} & 2 \pi^{2} & & & \\
4 \xi_{l} & & & 2 \pi^{2} & & \\
& & & & 2 \pi^{2} & \\
& & & & & 2 \pi^{2}
\end{array}\right)
\end{aligned}
$$

with determinant

$$
\operatorname{det} C=2 \pi^{4}\left(\pi^{2}-8 \xi^{2}\right)^{2}
$$


Substituting

$$
\begin{aligned}
\partial_{x} R & =r_{2} \cos \theta 2 \\
\partial_{y} R & =r_{1} \cos \theta 1 \\
\partial_{x} I & =r_{2} \sin \theta 2 \\
\partial_{y} I & =r_{1} \sin \theta 1
\end{aligned}
$$

and integrating over all components of $v$ yields the average pinwheel density

$$
\left\langle\rho_{l}\right\rangle=\pi \sqrt{1-\frac{8}{\pi^{2}} \xi^{2}}
$$

for an ensemble of planforms defined by the set of wavevector directions $l$. Since the anisotropy ranges within $0 \leq \xi \leq 1$, the pinwheel density (5.54) is confined by $1.36 \lesssim\left\langle\rho_{l}\right\rangle \leq \pi$. Eq. (5.54) appears to be consistent with the result from Fig. 5.2 showing that already for intermediate orders of $n$ averaging over $l$ leads to pinwheel densities smaller but close to $\pi$. That averages are smaller than $\pi$ is explained by the upper bound of $\left\langle\rho_{l}\right\rangle$ implying an upper bound also for its average. That they are, in fact, not much smaller than $\pi$ is already suggested from the 'relativistic' form of the dependency of the pinwheel density $\left\langle\rho_{l}\right\rangle$ on the anisotropy $\xi$ in (5.54). Even moderate anisotropies up to, e.g., $\xi_{l}=0.3$ result in pinwheel densities $\left\langle\rho_{l}\right\rangle>3$.

\subsubsection{Distribution of planform anisotropies}

What is the distribution of anisotropies $\xi$ in the large $n$ limit? To address this question consider the ensemble defined by the different sets $l=\left(l_{0}, \ldots, l_{n-1}\right)$. From now on, \langle\rangle shall denote the expectation value over this ensemble. In the following we assume that the distribution of the vector anisotropies $\vec{\xi}$ is isotropic and Gaussian in the large $n$ limit. The isotropy follows from the rotation symmetry of the model equations. It implies that

$$
\langle\vec{\xi}\rangle=0
$$

The Gaussian statistic is due to the fact that by its definition (5.43), the vector anisotropies $\vec{\xi}$ results from various wavevectors $l_{j} \mathbf{k}_{j}$ with pairwise independent directions $l_{j}$. The distribution thus reads

$$
p(\vec{\xi})=\frac{1}{\pi v_{\xi}} \exp \left(-\frac{\vec{\xi}^{2}}{v_{\xi}}\right)
$$


with variance $v_{\xi}$ given by

$$
\begin{aligned}
v_{\xi} & =\left\langle\vec{\xi}^{2}\right\rangle \\
& =\frac{1}{16 n^{2}} \sum_{j j^{\prime}} \mathbf{k}_{j} \mathbf{k}_{j^{\prime}}\left\langle l_{j} l_{j^{\prime}}\right\rangle \\
& =\frac{1}{16 n^{2}} \sum_{j} \mathbf{k}_{j}^{2} \\
& =\frac{\pi^{2}}{4 n}
\end{aligned}
$$

where $\left\langle l_{j} l_{j^{\prime}}\right\rangle=\delta_{j j^{\prime}}$. The probability density for $\xi$ follows from the distribution of the vector anisotropy $\vec{\xi}$ by

$$
\begin{aligned}
p(\xi) & =2 \pi \xi p(|\vec{\xi}|) \\
& =\frac{8 n}{\pi^{2}} \xi \exp \left(-\frac{4 n}{\pi^{2}} \xi^{2}\right), \quad \xi \geq 0
\end{aligned}
$$

where in the first equation the prefactor accounts for the change to the one-dimensional distribution of the modulus $|\vec{\xi}|$.

\subsubsection{Pinwheel density in the large $n$ limit}

Distribution (5.58) for the anisotropy $\xi$ translates with Eq. (5.54) into the distribution of pinwheel density $\rho$. This distribution is obtained from (5.58) by

$$
p(\rho)=p(\xi(\rho))\left|\frac{d \xi}{d \rho}\right|
$$

by means of a coordinate transform $\xi \rightarrow \rho$. With $\xi=\sqrt{\left(\pi^{2}-\rho^{2}\right) / 8}$ (Eq. 5.54) this leads to the pinwheel density distribution

$$
p(\rho)=\frac{n}{\pi^{2}} \rho \exp \left(-\frac{n}{2 \pi^{2}}\left(\pi^{2}-\rho^{2}\right)\right), \quad 0 \leq \rho \leq \pi,
$$

valid in the large $n$ limit. The expectation value of the pinwheel density follows as

$$
\begin{aligned}
\langle\rho\rangle & =\int_{0}^{\pi} d \rho \rho p(\rho) \\
& =\pi-\frac{e^{-\frac{n}{2}} \pi^{\frac{3}{2}} \Phi_{i}\left(\sqrt{\frac{n}{2}}\right)}{\sqrt{2 n}}
\end{aligned}
$$


where $\Phi_{i}$ is the imaginary error function. In the limit $n \rightarrow \infty$, the fraction vanishes yielding the average pinwheel density

$$
\lim _{n \rightarrow \infty}\langle\rho\rangle=\pi .
$$

This proves the convergence of pinwheel densities towards $\pi$ for large $n$ that has been suggested from Fig. 5.2p.

Moreover, the pinwheel density becomes $\delta$-distributed at $\langle\rho\rangle=\pi$ in the large $n$ limit as its variance $v$ converges towards 0 . Having

$$
\begin{aligned}
\left\langle\rho^{2}\right\rangle & =\int_{0}^{\pi} d \rho \rho^{2} p(\rho) \\
& =\pi^{2} \frac{2 e^{-\frac{n}{2}}+n-2}{n}
\end{aligned}
$$

which goes to $\pi^{2}$ for $n \rightarrow \infty$, one finds

$$
\begin{aligned}
v & =\left\langle\rho^{2}\right\rangle-\langle\rho\rangle^{2} \\
& =0
\end{aligned}
$$

suggesting that for large $n$ not only the average pinwheel densities $\langle\rho\rangle$ but the density $\rho$ of almost every single realization is close to $\pi$. This is consistent with the decrease of the SD $s$ for increasing $n$ observed for the distributions of pinwheel densities $\rho$ in synthesized realizations of planforms (Fig. 5.2p).

\subsection{Local pinwheel statistics}

We next asked whether in addition to the average pinwheel density the model also predicted the spatial variation and nearest neighbor distributions of pinwheels depicted in Fig. 4.10 and 4.11 .

\subsubsection{Count statistics}

In analogy to Fig. 4.10a, we calculated pinwheel densities $\rho$ in regions of various size $A$ in planforms with $n=20$ active modes. Fig. 5.3a shows that pinwheel densities $\rho$ varied considerably in regions of a few hypercolumns, and became closer to the average density $\langle\rho\rangle$ only in large regions. This quantitatively agreed to the maps from the visual cortex, as shown by the comparison of the SD $s$ and number variance $v$ in Fig. $5.3 \mathrm{~b}$. For the planforms, both quantities were calculated as in Fig. 4.10 with the count variance given by $v=\langle m-\langle m\rangle\rangle^{2}$ where $m$ is the number of pinwheels in an area $A$ and \langle\rangle denoting average over regions of size $A$. The SD $s$ 

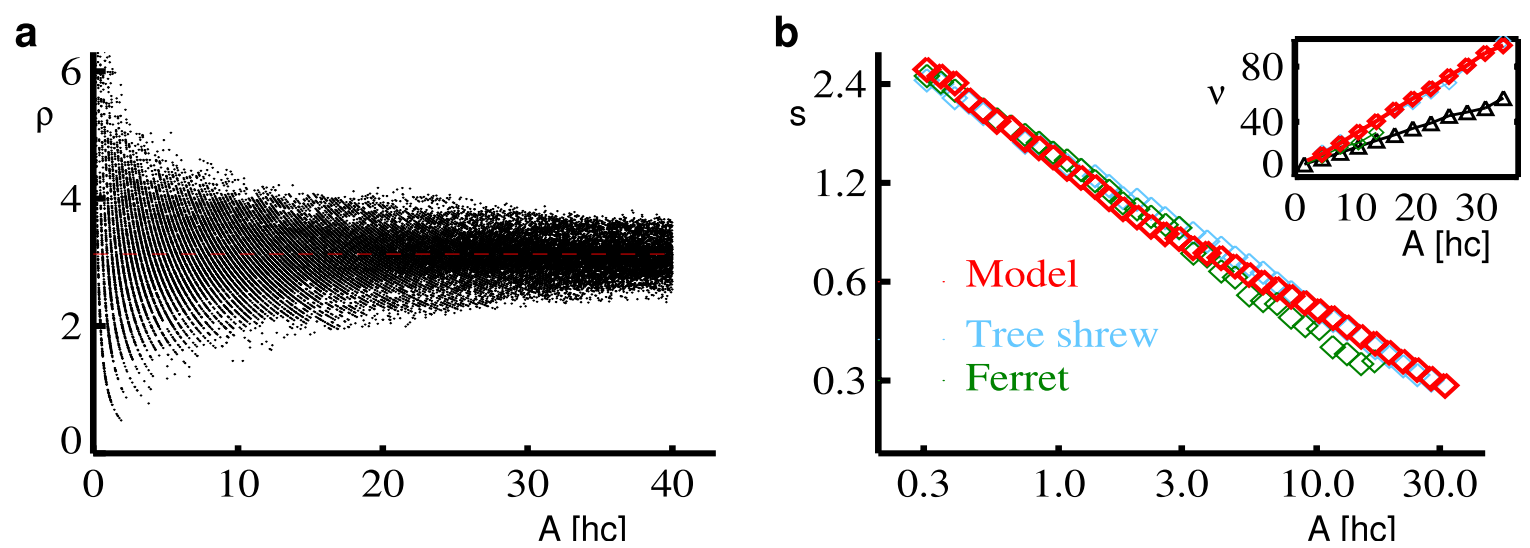

Figure 5.3: Spatial organization of pinwheels in the model for intermediate interaction range. a, Pinwheel densities $\rho$ in regions of various size $A$ (in units of hyper columns $\Lambda^{2}$ ). Regions were randomly sampled on planforms (5.23) (order, $n=20$; number of realization, $N=26$; 1000 regions drawn per hyper column increment). For comparison with experiment, planforms were confined to the geometry of tree shrew maps. $\mathbf{b}, \mathrm{SD} s$ and number variance $v$ of pinwheel densities $\rho$ from a compared to both quantities from tree shrew and ferret (Fig. 4.10). Note the excellent correspondence between the model and experiment and the deviation from the random map ensemble (black triangles).

decreased approximately proportional to $\sqrt{A}$ implying that $v$ increased as $c A\langle\rho\rangle$. The proportionality factor was $c \approx 0.9$ which agreed well with the factor observed in the visual cortex and which was very different than for the ensemble of phase shuffled maps (black triangles in 5.3p).

\subsubsection{Nearest neighbor statistics}

Another important characteristics of pinwheel patterns are the distances $h$ of a pinwheel to its nearest neighbors. Since pinwheels are characterized by a topological charge, three distances are of particular interest: the distance $h_{++,--}$to the nearest pinwheel of equal sign, the distance $h_{+-,-+}$to the nearest pinwheel of opposite sign, and $h_{t o t}$, the smallest distance to any other pinwheel. The distance $h_{t o t}$ is always the smaller of the two other distances. The histograms of these distances are statistics sensitive to the local arrangement of pinwheels.

Pinwheel nearest neighbor distances were very similar to those for the tree shrew and ferret as shown in Fig. 5.4. Distances were calculated for the same $n=20$ planforms as in Fig. 5.3 with methods identical to those used for the experimental data (Fig. 4.11). For these solutions, distances $h_{\text {tot }}$ to the next neighbor of arbitrary charge were mostly smaller than half the column spacing $\wedge$ (Fig. 5.4a). Very small distances occurred less frequently than intermediate distances. The distribution had a clear peak at $\approx 0.4$ defining a typical pinwheel distance of this value. As 

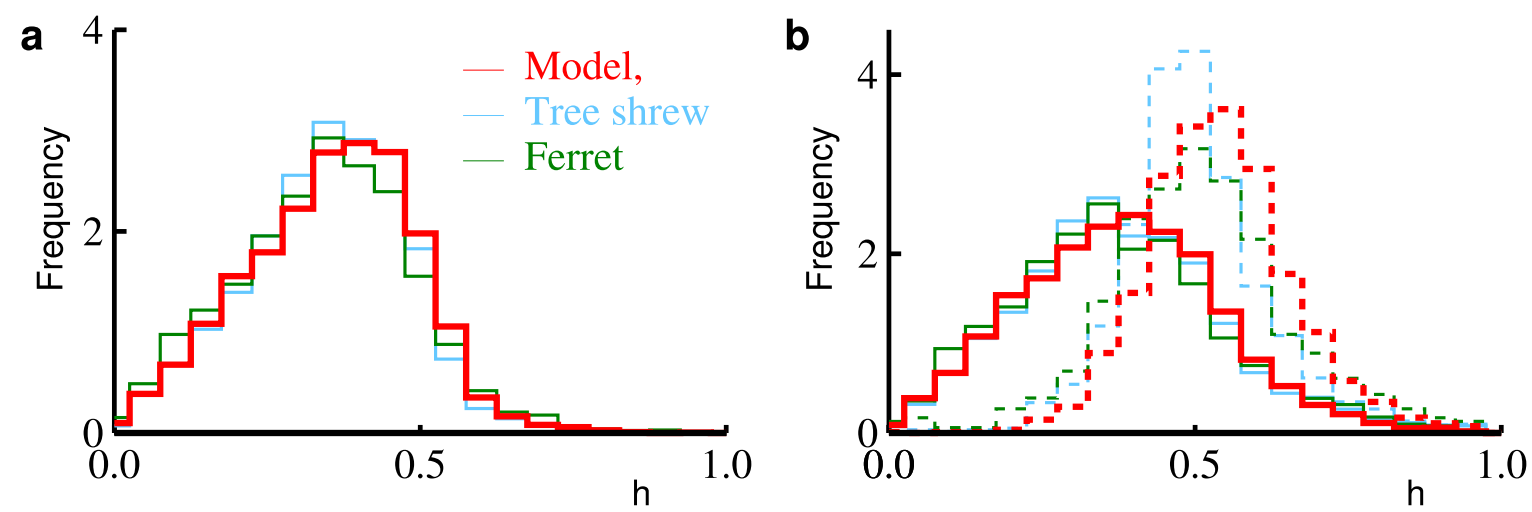

Figure 5.4: Nearest neighbor distances of pinwheels in the model for intermediate interaction range. a, Histogram of distances $h_{\text {tot }}$ to the next pinwheel of arbitrary topological charge for the planforms from Fig. 5.3 compared to those for the tree shrew and ferret (histograms normalized; distances in units of $\Lambda)$. b. Histograms for opposite charge $\left(h_{+-,-+}\right.$, solid lines) and equal charge $\left(h_{++,--}\right.$, dashed lines). Note the excellent agreement with tree shrew and ferret.

revealed by a comparison with Fig. $5.4 \mathrm{~b}$, most nearest neighbors were of opposite sign. Distances between equally charged pinwheels were considerably larger with peak at $\approx 0.55$ and only a negligible fraction of the distances being smaller than $\lesssim 0.2 \wedge$. Each of these distributions was very similar to the analog distribution obtained for the tree shrew and ferret (thin lines in Fig. 5.4a, b; from Fig. 4.11). Not only did they agree in their center and width, but also displayed a virtually identical overall shape. These findings remain valid for other choices of $n>13$ $(\sigma \gtrsim 2.0)$. Thus, in this regime, the model predicts all aspects of pinwheel organization found to be universal in the visual cortex: The average pinwheel density, the regional variation of local density and the distribution of distances between adjacent pinwheels.

\subsection{Pinwheel densities away from criticality}

It is obviously difficult to gauge the value of the bifurcation parameter $r$ for the development of the orientation map in the visual cortex, and the possibility that the system is in a regime further away from the bifurcation point must not be neglected. It is unclear, however, whether the results valid close to criticality established in the previous Section 5.3 generalize to the regime further away from the bifurcation point. To assess the robustness of these results it is therefore important to study the regime $r>0$.

In fact, a regime $r>0$ is suggested from the following considerations. First, at least for the tree shrew, the layout of the pattern along the boundary suggests a rather short ranging influence within a few column spacings $\Lambda$. (Fig. 2.1). This is, 
a
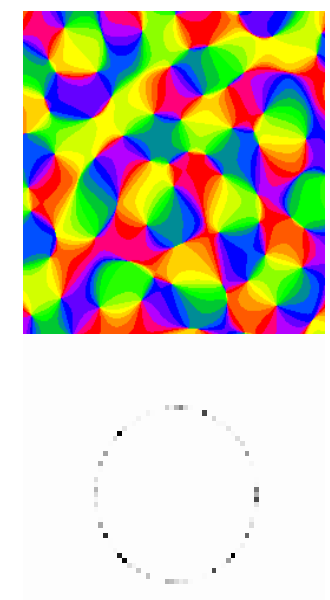

$t=3 \times 10^{2}$
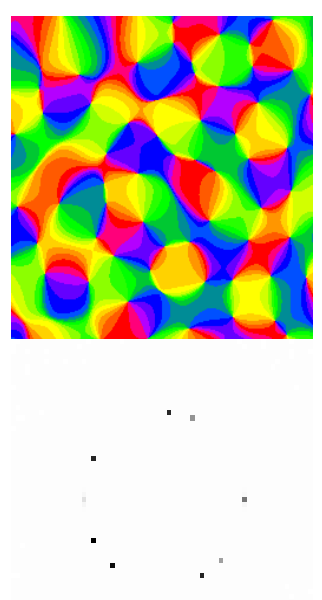

$t=3 \times 10^{4}$ b
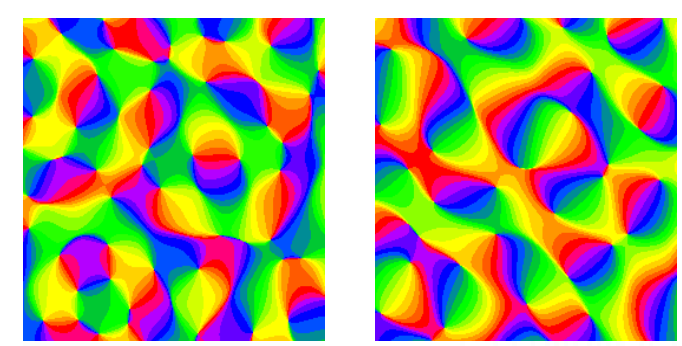

$t=3 \times 10^{2}$

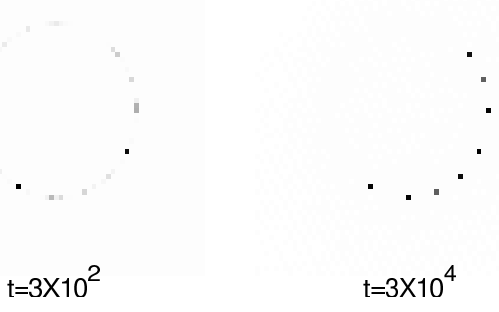

Figure 5.5: Two examples of orientation maps obtained at intermediate time $t=3 \cdot 10^{2}$ (left in a, b) and at late time $t=3 \cdot 10^{4}$ (right in $\mathbf{a}, \mathbf{b}$ ) with $t$ rescaled by $T=1 / r$. Integration of 5.1 and parameters as in Fig. 5.1. The upper row shows subregions of the angle map $\left(A=(4.25 \Lambda)^{2}\right.$, smoothed for illustration). Below each angle map the power spectrum of the entire map is depicted (origin of Fourier-space in center of annulus). At $t=3 \cdot 10^{2}$ unstable active modes are present. In a an example of a high pinwheel density map is depicted with almost equidistance of active modes on the critical circle. $\mathbf{b}$ shows an example of a low pinwheel density map with most modes confined to one half of the critical circle.

however, inconsistent with the stiffness of the solutions implied by $r \ll 1$. As mentioned above, only for $r>0$, the critical circle is sufficiently wide to allow for such sharp transitions in the layout. Second, even in orientation maps of intermediate size with aspect ratios of $\Gamma \approx 20-30$, large-scale inhomogeneities are observed that may be interpreted as different domains (see e.g. Fig. 4.1). Clearly, such domains would only occur in a regime further away from the bifurcation point, since owing to the width $\delta k \propto \sqrt{r}$ of the critical circle, the size of such a coherent structure is $\mathcal{O}(1 / \sqrt{r})$. Third, assuming that $r$ is not constant in time in a cortex permanently driven by visual stimuli, in the case $r$ were close to the bifurcation point, these fluctuations of $r$ could cause occasional transitions from maps of orientation selective neurons to unselective neurons. Such large scale fluctuations, however, were never observed in recordings nor do they appear very beneficial for vision.

Two representative solutions of Eq. (5.1) for $r=0.1$ are depicted in Fig. 5.5 at intermediate and late times $t$ (rescaled by (5.19). In transient states at $t=3 \cdot 10^{2}$ all active amplitudes were already confined to the critical circle, but not yet in a stable configuration. In the near stationary states at $t=3 \cdot 10^{4}$, in contrast, all finally active amplitudes were selected and the further development mainly consisted in the relaxation of the phases of these amplitudes. Whereas the final state depicted in 
Fig. 5.5a exhibited a high pinwheel density with almost equidistant active modes on the critical circle (small anisotropy $\xi$, Eq. (5.43)), a map of lower pinwheel density with most modes confined to one side of the spectrum (large anisotropy $\xi$ ) is shown in Fig. 5.5p.

\subsubsection{Transient states}

Transient states exhibited average pinwheel densities $\langle\rho\rangle$ very similar to those observed in the visual cortex in Chapter 4 . As shown in Fig. 5.6a, after an intermediate integration time $t=3 \cdot 10^{2}$ the average pinwheel density was $\langle\rho\rangle \approx \pi$ for various values of $r$ and interaction ranges $\sigma$. However, pinwheel densities did not mimic the fluctuation with $\sigma$ found for planforms (Fig. 5.6p), but appeared rather independent of $\sigma$. The deviation from the planforms was even more apparent for the variability $s$ of pinwheel densities $\rho$ across individual maps (SD, Fig. 5.6p).

The origin of this deviation from planform solutions is that this developmental stage still falls into the phase of nonlinear competition. The finally active modes have not been fully selected yet. Especially, the low pinwheel density realizations with active modes only in one half of the critical circle leading to high anisotropies $\xi$ occur only in much later stages. Therefore, pinwheel densities at this stage exhibit slightly higher values and less variation compared to planforms. Map development is further discussed in Chapter 6.

\subsubsection{Near stationary states}

The pinwheel densities observed for the near stationary states agreed for small values of $r$ well with the experimental results and those obtained for planforms. Fig. 5.6c shows the average pinwheel densities $\langle\rho\rangle$ for the same initial conditions as in Fig. 5.6a, but after $t=3 \cdot 10^{4}$. Above $r \geq 0.03$, however, a deviation of $\langle\rho\rangle$ relative to the planform densities became evident. Solutions for a given $r$ diverged stronger from the asymptotic density near criticality, $\langle\rho\rangle=\pi$, for larger interaction ranges $\sigma$. Also the variation $s$ followed the results for planforms only for small $r$ and interaction ranges $1.0 \leq \sigma \leq 2.0$ (Fig. 5.2 d), but was more pronounced for large values of $\sigma$ and $r$.

The interpretation of these findings is that at this stage the phase of nonlinear competition has come to an end. Planform-like solutions are selected, however, with a bias towards larger anisotropies $\xi$ and therefore to smaller average pinwheel densities and a larger variation (see Section 5.3). Different from planforms, for $r>0$ solutions do not appear to have degenerated energy anymore. Striving the amplitude expansion to higher than 3rd order may provide insights into an energy splitting or other mechanism controlling the selection of solutions. 
a
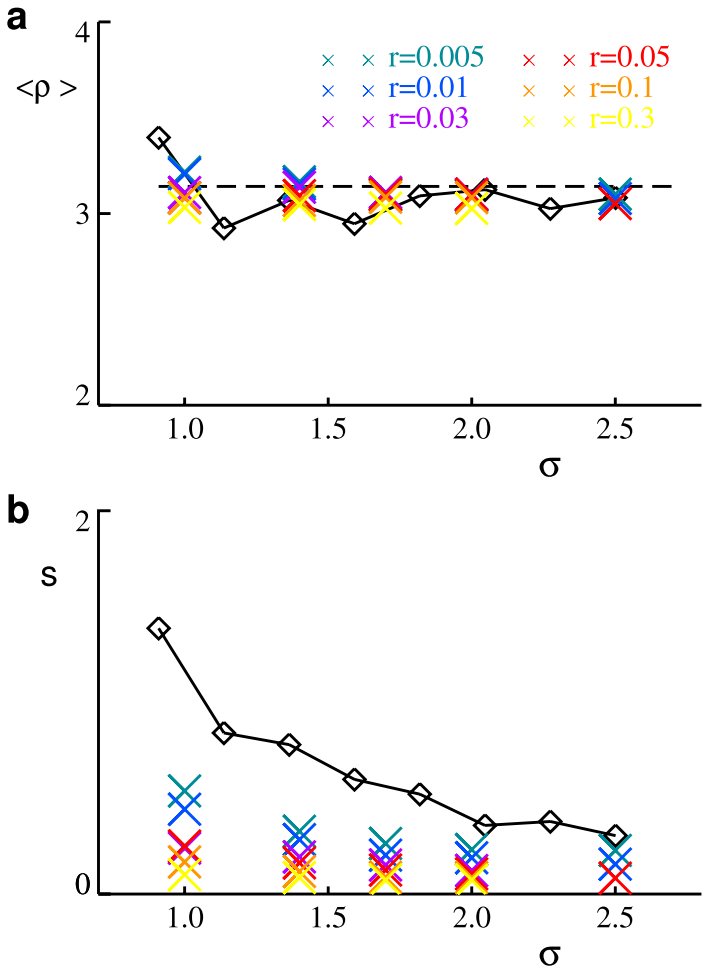
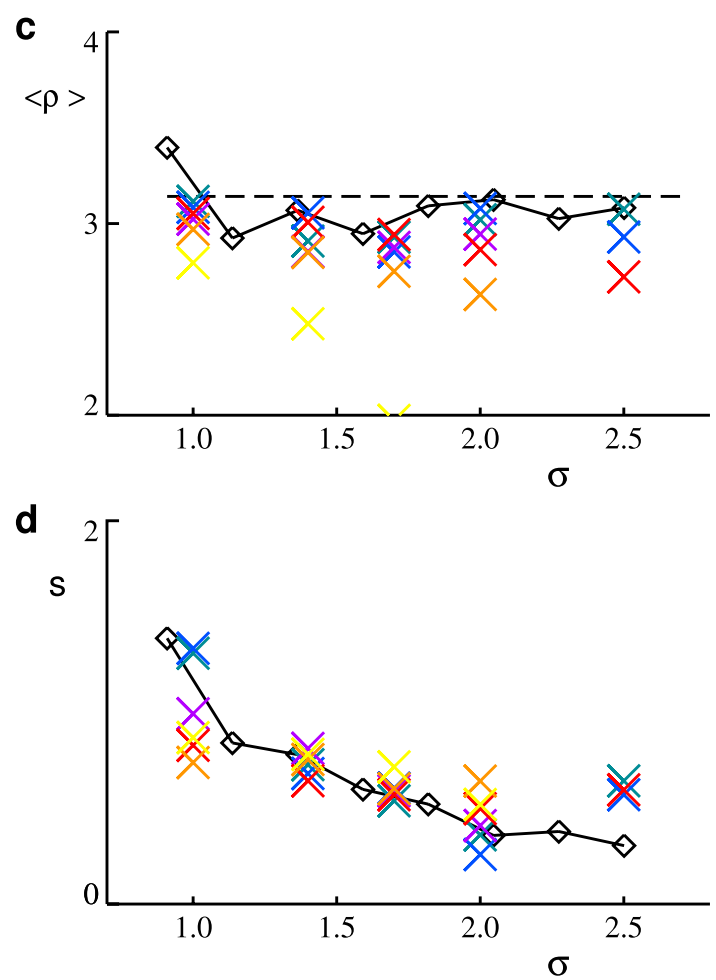

Figure 5.6: Pinwheel densities of the full model Eq. (5.1). a, Average pinwheel densities $\langle\rho\rangle$ for different values of bifurcation parameter $r$ and interaction range $\sigma$. Numerical solutions were obtained after $t=3 \cdot 10^{2}$ starting from the identical set $(\mathrm{N}=50)$ of initial conditions $z_{0}$ at $t=0$ for each parameter set (see Methods; mesh size, $128 \times 128$ using periodic boundary conditions; $g=0.98, \Gamma=21$ for $\sigma=1.0,1.4, \Gamma=24$ for $\sigma=1.7,2.0, \Gamma=25$ for $\sigma=2.5$ ). Pinwheel densities $\langle\rho\rangle$ of planform solutions (Fig. 5.2 shown at $\sigma=n / 1.5 \pi$ (diamonds). Their asymptotic density $\langle\rho\rangle=\pi$ is indicated by the dashed line. $\mathbf{b}$, SD $s$ of pinwheel densities. $\mathbf{c}, \mathbf{d}$, as in $\mathbf{a}, \mathbf{b}$, respectively, but at a much larger time $t=3 \cdot 10^{4}$ at which solutions are close to the attractor.

\subsubsection{Robust selection}

The importance of long-range connections for the selection of pinwheel densities is illustrated in Fig. 5.7. If long-range interactions are absent, low pinwheel density solutions are preferred and pinwheels annhilate after a short period of time [107 108]. In the presence of long-range interactions, pinwheel densities are robustly selected. Pinwheel densities and pinwheel dynamics during development are further studied in Chapter 6. 
a

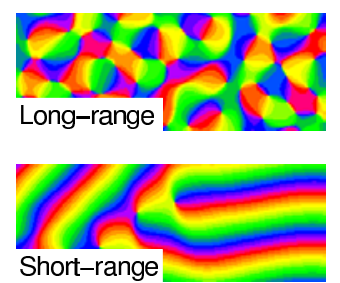

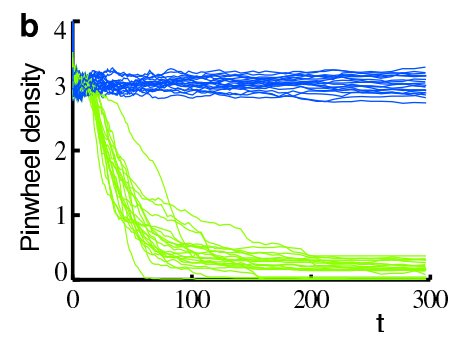

Figure 5.7: Robust dynamical pinwheel density selection. a, Upper map, $r=0.1, g=0.98$ and $\sigma=1.7$. Lower map, $r=0.1, g=2$. b, Pinwheel densities $\rho$ in the presence (blue) and absence (green) of longrange interactions $(N=30$, parameter as in a).

\subsection{Discussion}

The previous Chapter 4 identified several universal properties of pinwheel statistics in the visual cortex. This resembles a behavior found in many complex systems where structures within a particular universality class are selected by selforganization despite the often involved different microscopic dynamics [23]. In this chapter, we analyzed a model formalizing the idea of self-organization of the orientation map including only key features of the visual cortical organization.

The model showed that pinwheel densities close to $\pi$ are robustly selected if interactions between remote contour detectors are prevalent. Solutions closely resembled real orientation maps in the large parameter regime of sufficiently wide and strong nonlocal interactions $(\sigma \gtrsim 0.5 \Lambda, 0 \leq g<1)$. Otherwise, low pinwheel density solutions are preferred and pinwheels annhilate after a short period of time [107 108]. Near criticality $(r \ll 1)$, in the limit of large intracortical interaction range $\sigma$, the average pinwheel density converged to the fixed number $\langle\rho\rangle=\pi$. For intermediate ranges $1 \Lambda \lesssim \sigma \lesssim 3 \Lambda$, average pinwheel densities $\langle\rho\rangle$ were smaller but close to this limit value. For successively larger ranges $\sigma$, the pinwheel density approaches $\pi$ from below. Numerical solutions of the model using various values of $r$ confirmed these results. Both medium term and near stationary solutions exhibited for $1 \leq \sigma \leq 2.3$ similar average pinwheel densities $\langle\rho\rangle$ as for $r \ll 1$. Thus, in an apparently realistic parameter regime, the model agrees quantitatively with the average pinwheel density $\langle\rho\rangle$ in the tree shrew, galago and ferret.

We evaluated the spatial variation and nearest neighbor distributions of pinwheels and compared them to the experimental results from the previous Chapter 4. Pinwheel densities $\rho$ varied considerably in regions of a few hypercolumns, and converged towards the average density $\langle\rho\rangle$ only in large regions. This quantitatively agreed with the tree shrew and ferret maps, as demonstrated by the comparison of the SD and number variance. Moreover, also pinwheel nearest neighbor distances were very similar to those for the tree shrew and ferret. These findings remained valid within a large parameter regime $\sigma \gtrsim 2.0$ and $g<1$. Thus, in this regime, the model reproduces all aspects of pinwheel organization found to be universal in the visual cortex: The average pinwheel density, the regional variation of 
local density and the distribution of distances between adjacent pinwheels.

The invariance of the pinwheel density in the visual cortex is a non-local principle. A small region of cortex does not experience a hard constraint and may therefore deviate substantially from average. Rather, this principle applies to the network as a whole governing its organization on a large spatial scale. This suggests that long-range horizontal connections are crucial for this process. Indeed, the proposed model indicates that for the establishment of the observed pinwheel patterns, a prevalent contribution of non-local interactions is both necessary and sufficient. The latter explains the robustness of its occurrence in different species. The former explains why similar patterns were not often observed in examples of pattern formation from physics, since in most pattern forming systems interactions are local [3, 23]. It is worth mentioning that a non-local principle offers the possibility of fast and widespread organization and thus the development of a virtually mature map at an early stage. The development of the orientation map is studied in more detail in the following chapter. 


\section{Predicted pinwheel kinetics during development}

\subsection{Motivation}

So far, most recordings of cortical maps are carried out at a single point in time, often in the adult visual cortex. Chronic recordings that allow for measurements over many hours, days or weeks are technically possible, but challenge experimentalists. Nevertheless, it is expected that chronic recordings will be important in order to understand the dynamics of the neuronal network during development. In enhancing the efficiency of upcoming experiments, model studies shall contribute an important part. Properties on which to focus in experiment are suggested by the model. The time scale, for instance, on which network changes occur is a useful quantity to know when endeavoring an experiment. In the model study in this chapter, we focus on the dynamics of pinwheels during development. The methods for analyzing pinwheels in experimental data developed in Chapter 4 can be extended to data from chronic experiments. A first approach to this is undertaken in Chapter 8 . Thus, a direct test of the analyses presented in this section is expected to be possible in the near future.

We begin this chapter by analyzing the changes of the layout of the orientation map over time using the generalized Swift-Hohenberg model Eq. (5.1). We assess the evolution of various pinwheel statistics during development including pinwheel density, nearest neighbor distributions, pinwheel creation and annihilation rates, survival probabilities, pinwheel motion and pinwheel speed. We identify two different regimes, the regime of early nonlinear selection in which the development is largely independent of the bifurcation parameter $r$, and a late regime in which pinwheel statistics depend on $r$. This suggest to derive the relevant time scales of the early nonlinear phase from weakly nonlinear stability analysis. Finally, we consider the development of orientation maps in hard wall boundary conditions. 


\subsection{Methods}

The model and its numerical integration is described in Section 5.2 of the previous chapter.

\subsubsection{Pinwheel tracking}

To track pinwheels during the evolution of the orientation map, states were monitored at times $t_{i}$ separated by exponentially decreasing time intervals $\Delta t_{i}=t_{i}-t_{i-1}$ in order to account for the various temporal scales the dynamics encounters from the primary instability at $t=0$ to the very slow development near the attractor at $t=1 \cdot 10^{6}$. Usually, 300 maps were sampled at times

$$
t_{i}=\frac{t_{f}}{e^{10}-1}\left(e^{\left(10 t_{i}^{\prime} / t_{f}\right)}-1\right)
$$

with $t_{i}^{\prime}$ equally distributed between the initial time $t_{0}=0$ and the final time $t_{f}$, where $t_{f}=3 \times 10^{4}$, or in long-run simulations $t_{f}=1 \times 10^{6}$, respectively. All results were checked by using constant time intervals $\Delta_{i}$ with various temporal resolutions. Pinwheel locations were identified at each time $t_{i}$ by the crossings of the zero contour lines of the real and imaginary part of the orientation map. Pinwheels were tracked over time based on their similar locations in maps at successive times $t_{i}$ and $t_{i+1}$. Since pinwheels move continuously, there is a time interval $\Delta t_{i}=t_{i+1}-t_{i}$ for which all pinwheels move less than a given distance $\Delta x$. A pinwheel differing in position by less then $\Delta x$ between $t_{i}$ and $t_{i+1}$ was considered identical. If more than one pinwheel had a smaller distance than $\Delta x$ the one with the smallest distance was chosen (a case which most often occurred before annihilation when two pinwheels approached each other). If no corresponding pinwheel was found within $\Delta x$ at subsequent time $t_{i+1}$ it was considered as annihilated. If a pinwheel at $t_{i+1}$ could not be assigned to one at $t_{i}$ it was considered as created. With a choice of $\Delta x=0.2 \Lambda$, the above exponential temporal sampling provided a sufficient resolution of pinwheel movements.

\subsubsection{Boundary conditions}

To integrate equation (6) with boundary conditions (6.41) on a disk (Section 6.6), a circular ramp in the bifurcation parameter $r$ was used, maintaining the region surrounding the circle at a subcritical value thereby suppressing the amplitude of the pattern. A similar treatment of boundary conditions (6.41) has been used in numerical solutions of the Swift-Hohenberg equation [74] and of the Navier-Stokes equation [28] where it has been shown successfully in comparison with experiment. A 
boundary term was defined by

$$
B(\mathbf{x})=-\operatorname{br} \Theta\left(\left|\mathbf{x}-\mathbf{x}_{0}\right|-R\right)
$$

with $R$ the radius of the disk centered at $\mathbf{x}_{0}$ in the simulated quadratic region and $b>1$ controlling the strength of the suppression at and outside the boundary. The ramping was implemented in the Fourier domain by

$$
\tilde{N}_{B}(\tilde{z}, t)=\tilde{N}(\tilde{z}, t)+\tilde{B} * \tilde{z}
$$

as an additional contribution to the force term in Eq. (5.9). To enforce boundary conditions (6.41) one may choose $b \gg 1$. However, this would reduce the step size of time marching drastically since the implementation of $(6.3)$ is explicit. In numerical tests using various strengths $b$, a sufficient impact of the imposed constraints on the pattern was obtained even for $b \geq 2$. In typical simulations ranging over large times $\left(t=1 \times 10^{6}\right)$ a value of $b=3$ was used. Wrap around effects induced by the pseudo spectral treatment of the nonlocal interactions in the nonlinear part (5.3) were avoided by choosing the region inside the boundary sufficiently small. With an aspect ratio of $\Gamma=24.5$ and an interaction range $\sigma \leq 2.5 \Lambda$, this was achieved with a diameter of the disk covering $7 / 8$ of the total width of the simulated quadratic region (mesh size $128 \times 128$ ). Initial conditions were Gaussian random fields with an average amplitude of $A=1 \times 10^{-4} \sqrt{r}$ throughout the entire simulated region.

\subsection{Development of orientation maps}

A representative example of a developing orientation map is presented in Fig. 6.1. After $t=10^{2}$, the state already resembled an orientation map observed in the visual cortex. The initially broad spectrum was narrowed due to the strong damping of the linear part Eq. (5.2) further away from the critical circle $|\mathbf{k}|=k_{c}$. On the critical circle, modes grew initially exponential with rate $r$ until their amplitudes were of order $\sim r^{1 / 2}$. For the choice of $r$ and the amplitude of the initial conditions used, this phase typically ended at $t \approx 10^{1}$ (Fig. 5.1). The subsequent phase of nonlinear competition among modes lead to the development of isolated amplitudes distributed around the critical circle at $t=10^{2}$, and to a stable configuration of amplitudes at $t=10^{4}$. The solution consisted of a finite number of active modes as predicted by the amplitude formalism (see Chapter 5 and 2). Different from the planform solutions, however, phases $\phi_{i}$ of the amplitudes $A_{j}=\left|A_{j}\right| e^{i \phi_{j}}$ were not degenerate anymore. In fact, phases $\phi_{i}$ still changed after $t=10^{4}$ and complete stationarity was not always reached even at $t=10^{6}$.

The amplitude formalism provides an approximate description for the second phase of mode competition. Within this framework modes develop on a time scale 

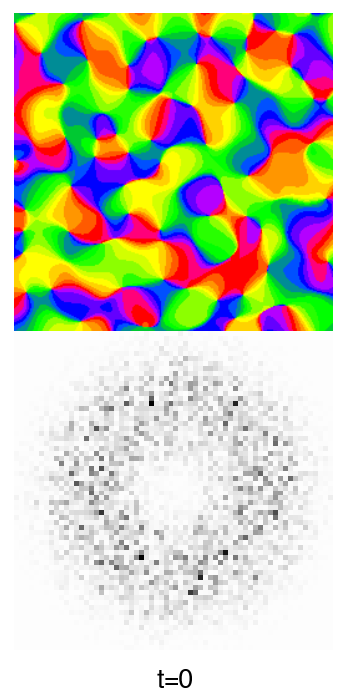
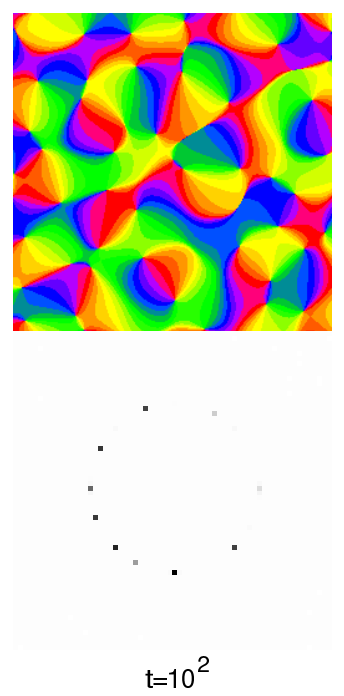
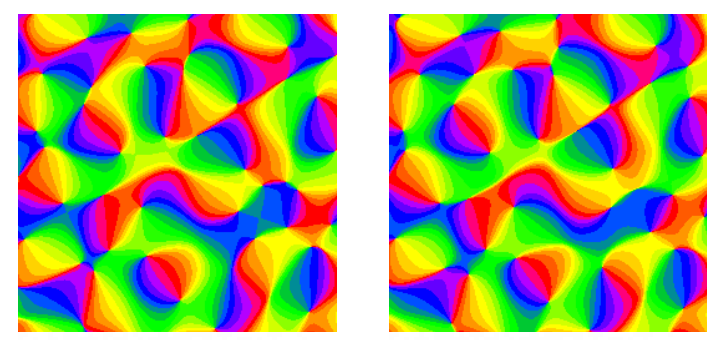

$t=10^{4}$
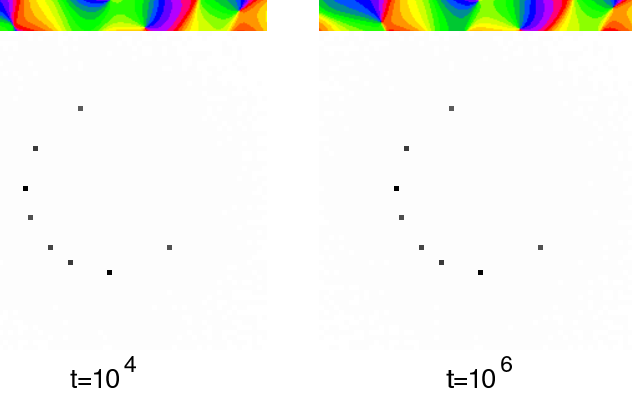

Figure 6.1: A developing orientation map. A band-pass filtered Gaussian random map as initial condition at $t=0$ was advanced until $t_{f}=10^{6}$. Integration of Eq. (5.1) and parameters as in Fig. 5.1 $(r=0.1, g=0.98, \sigma=1.7 \Lambda, \Gamma=17)$. The upper row shows a subregions of the angle map at initial, final and intermediate times $\left(A=(4.25 \Lambda)^{2}\right.$, smoothed for illustration) with beneath the power spectrum of the entire map (origin of Fourier-space in center of annulus). Note that the map is considerably stable already after $t=10^{2}$.

of $\mathcal{O}(r)$. Thus, during the first two phases orientation maps might approximately develop on time scale $\mathcal{O}(r)$. Stable solutions of the amplitude equations of third order are degenerate in phases $\phi_{j}$. Since the next correction is of 5 th order the adjustment of phases will happen on a time scale of at least $\mathcal{O}\left(r^{2}\right)$. This suggests that in maps the selection of phases $\phi_{j}$ takes place on a much larger time scale consistent with the long term dynamics depicted in Fig. 6.1 and in the following figures. To compare results obtained for different values of $r$ during the phase of mode competition, they were presented using the rescaled time $t$ (Eq. (5.19)), as in previous sections.

A more quantitative view on the map development governed by Eq. (5.1) is revealed by tracking the similarity across different stages of development. For this purpose consider the time dependent cross-correlation

$$
C\left(t, t^{\prime}\right)=\sqrt{\frac{\operatorname{Re}\left(\int d^{2} x z(\mathbf{x}, t) \bar{z}\left(\mathbf{x}, t^{\prime}\right)\right)}{\sqrt{\int d^{2} x|z(\mathbf{x}, t)|^{2} \int d^{2} x\left|z\left(\mathbf{x}, t^{\prime}\right)\right|^{2}}}}
$$

between the map at time $t$ and the map at a fixed reference time $t^{\prime}$. Fig. 6.2 shows the correlation $C$ for three different reference times $t^{\prime}$ and for three values of $r$. Each cross-correlation was averaged over $N=10$ different solutions.

For $r=0.1$ (red curves), maps developed rapidly during the early phase and the 
correlation between the initial and the developing map dropped considerably before $t=10^{3}$ (dashed curve). Interestingly, however, even at very late stages, maps did not become uncorrelated from the initial maps, but maintained a correlation of $C \approx 0.2$. Moreover, very late maps at $t=10^{6}$ were with $C \approx 0.5$ still moderately correlated with their early states at $t=10^{2}$ (dashed-dotted curve), a time still at the beginning of the phase of mode competition. When the modes reached their final configuration at $t=10^{4}$ maps were already very similar to their very late states at $t=10^{6}$. During phase relaxation, correlations did not change substantially indicating that on average, phases experience only small changes, at least until $t=10^{6}$ (solid curve). Thus, largest changes occurred before $t \approx 10^{3}$. After $t \approx 10^{4}$, maps were basically stable. The map layout strongly resembles the final layout even before that time, after $t=10^{2}$ as shown by the representative example in Fig. 6.1 (cross-correlation between maps at $t=10^{2}$ and $t=10^{6}, C=0.45$ ). Consistent with these results, several experimental studies observed a persistence of the basic layout of the orientation map during development. Experimental paradigms for testing the predicted dynamical changes during development will be proposed at the end of this chapter.

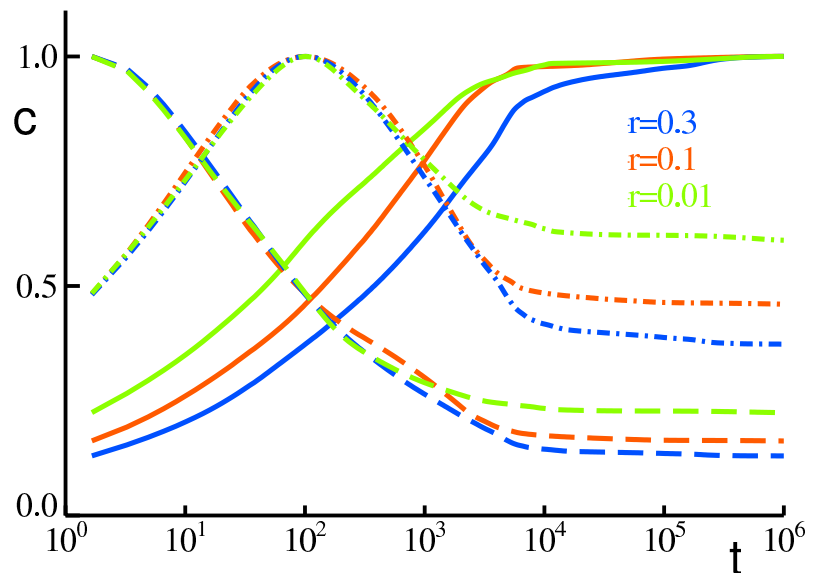

Figure 6.2: Quantified changes during map development. Cross-correlation $C\left(t, t^{\prime}\right)$ (Eq. (6.4) between the map and the map at fixed reference times $t^{\prime}=$ $10^{0}$ (dashed lines), $t^{\prime}=10^{2}$ (dasheddotted), $t^{\prime}=10^{6}$ (solid) for different values of $r$. Each curve represent an average over $N=10$ cases. Parameters as in Fig. 5.1 Note that already after $t \approx 10^{2}$, maps are considerably correlated with their very late states at $t=10^{6}$.

A dependence of map stability on $r$ was observed especially during the intermediate and late stages of development. Solutions obtained for different values of $r$ showed identical time courses of $C(t)$ during the initial phase of development (dashed curves). Approximately between $t=10^{3}$ and $t=10^{4}$, a dependence on $r$ became apparent as highlighted by the dashed-dotes curves. Whereas for small $r$, solutions remained relatively similar to the early state at $t=1 \cdot 10^{2}$ during this stage of development $(C \approx 0.6)$, they diverged stronger from the early state for large $r$ as indicated from the decrease of $C$ below 0.4 . At late stages $t>1 \cdot 10^{4}$, correlations stabilized. Consistent with a faster phase dynamics for large $r$, changes during this phase were stronger for solutions with a larger value of $r$ (solid curves).

Following these results, the development of orientation maps may be subdivided into three stages. First, a phase of linear growth taking place for $0 \leq t \leq 10^{1}$ when 
amplitudes near the critical circle grow until reaching their maximum. Second, the phase of nonlinear competition among modes between $10^{1}<t<10^{4}$ when the finally active amplitudes are selected. Third, the relaxation of phases of the selected amplitudes for $t>10^{4}$. This defines two time scales central for the study in this section: The end of the linear phase denoted by $t_{0} \approx 10^{1}$ and the end of mode competition called the cross-over phase $t^{*} \approx 10^{4}$.

\subsection{Development of pinwheel statistics}

Typically, pinwheel densities fluctuated considerably during development. Fig. 6.3 shows a representative example of the time course of pinwheel density $\rho(t)$ in an orientation map developing from a Gaussian random field at $t=0$ to $t=3 \cdot 10^{4}$. Between $t=1 \cdot 10^{0}$ and $t=1 \cdot 10^{3}$ the pinwheel density $\rho(t)$ varied between 2.8 and 3.3 with fluctuations occurring on various temporal scales. During the late phase at $t>1 \cdot 10^{4}$ that is characterized by the relaxation of phases fluctuations of $\rho(t)$ diminished considerably.

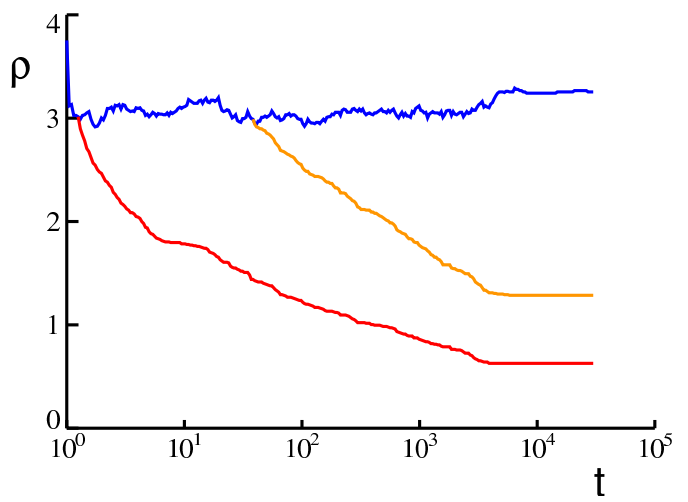

Figure 6.3: Tracing pinwheels during development in model (5.1). Time course of pinwheel density $\rho(t)$ of an orientation map developing from band-pass filtered white noise at $t=0$ to a near final state at $t=3 \times 10^{4}$ (blue curve). Integration of 5.1 and parameters as in Fig. 5.1. Pinwheels were counted and tracked in a square of size $12.75 \wedge$ over 300 intermediate solutions. The survival fraction $\mu_{\tau}(t)$ of pinwheels present at $t=\tau$

not annihilated until $t$ is shown for $\tau=$ $1 \times 10^{0}$ (red) and $\tau=5 \times 10^{1}$ (orange).

The fluctuation of the pinwheel density $\rho$ was mainly due to pinwheel annihilation and creation processes happening frequently during development. From a set of pinwheels present at time $t=\tau$ only the fraction $\mu_{\tau}(t)$ survives until time $t \geq \tau$. To calculate $\mu_{\tau}(t)$, pinwheels were tracked throughout the course of development (see Methods, Section 6.2). Fig. 6.3 shows the survival fractions $\mu_{\tau}(t)$ of pinwheels for $\tau=1 \cdot 10^{0}$ and $\tau=5 \cdot 10^{1}$. Both fractions decayed rapidly during the phase of nonlinear competition. After cross-over to pure phase dynamics at $t^{*} \approx 1 \cdot 10^{4}$, pinwheel annihilation occurred much less frequently consistent with the reduced strength of density fluctuations in this regime. Pinwheel annihilation and creation processes are further studied below (Section 6.4.3). 

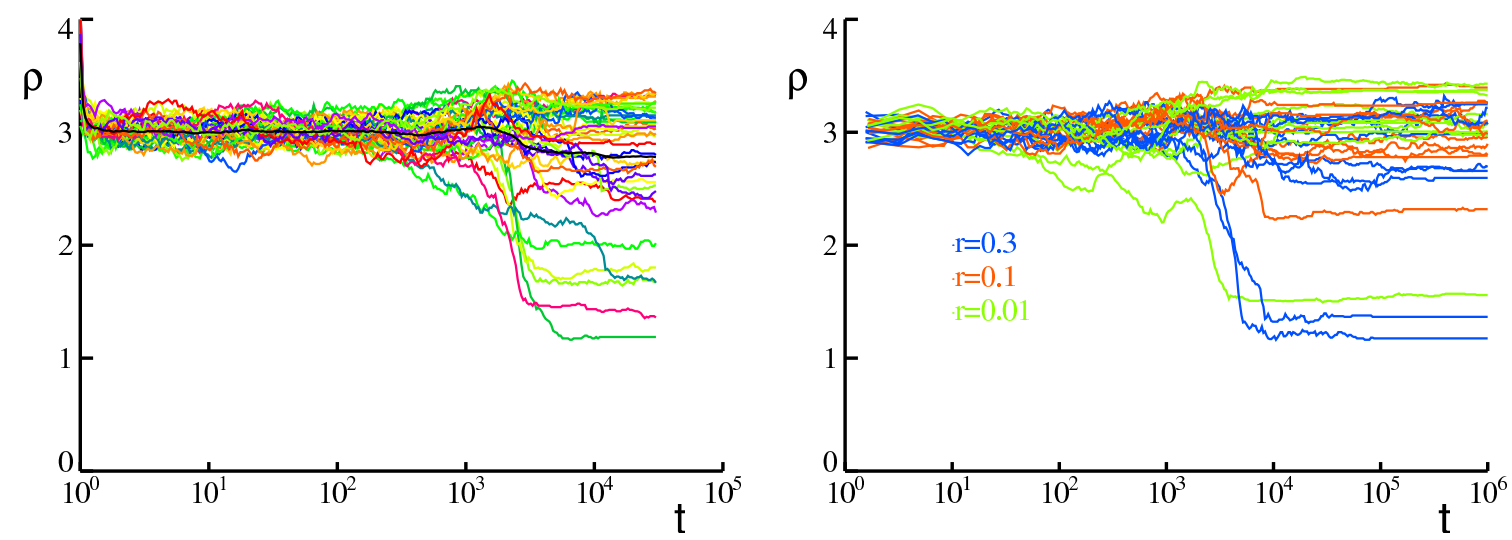

Figure 6.4: Pinwheel densities during development. a, Pinwheel density trace $\rho(t)$ for different random initial conditions ( $N=40$, parameter as in Fig. 6.1 and Fig. 6.3. The average trace is represented by the black line. Until $t \approx 3 \cdot 10^{2}$, all traces were confined to the small range $2.8<\rho<3.3$. Thereafter, traces diverged and reached near final levels at the cross-over time $t^{*} \approx 1 \cdot 10^{4}$. b. Traces $\rho(t)$ obtained for different values of the bifurcation parameter $r(N=10)$. Solutions were advanced to $t=1 \cdot 10^{6}$ (rescaled time). Note that independent from $r$ traces diverge during the same period just before $t=t^{*}$. In the late stage $t>t^{*}$, pinwheel densities exhibited much smaller fluctuations.

\subsubsection{Pinwheel density}

Fig. 6.4 shows the evolution of pinwheel densities $\rho(t)$ for a large set of different initial conditions. Up to $t \approx 3 \cdot 10^{2}$, pinwheel densities $\rho$ were generally confined between 2.8 and 3.3. However, later on, the densities diverged starting from various points in time and with rates of change depending on initial conditions. The near steady-state distribution reached at cross-over time $t=t^{*}$ was confined to an interval between $\rho=1$ and $\rho=3.5$ similar to the distribution obtained for planforms [105]. The low density solutions exhibit large anisotropies $\xi$, i.e. have most active amplitudes located in a hemifield (Section 5.3). All transient pinwheel densities $\rho(t)$ for $t \leq 3 \cdot 10^{2}$ were close to $\pi$, consistent with Fig. 5.6 showing an average pinwheel density of $\langle\rho\rangle \approx \pi$ at $t=3 \cdot 10^{2}$. For most cases, also the final density was close to $\pi$, but their average was, consistent with the results from Fig. 5.6. slightly reduced due to the presence of solutions with large anisotropy $\xi$ at large times.

How does the described dynamics of pinwheel densities depend on the bifurcation parameter $r$ ? Fig. 6.4p shows that near final values of pinwheel densities $\rho$ developed at $t \approx 1 \cdot 10^{4}=t^{*}$ independent of $r$ suggesting that the phase of nonlinear competition follows a similar type of dynamics for different $r$. However, other than the time course of selection, the selected ensemble did depend on $r$ with a tendency towards low density solutions for large $r$ consistent with the results from 
Fig. 5.6 (for discussion, refer to Section 5.5). Owing to the ongoing phase relaxation, pinwheel densities continued to change after $t=t^{*}$, albeit with reduced amplitudes of fluctuations and as expected with faster changes for larger $r$. Thus, whereas some details of the pinwheel dynamics depend on $r$, the time scale $t^{*}$ governing the primary development appears insensitive for different $r$.

How well do pinwheel densities at reference time $t^{\prime}$ predict densities at time $t$ ? Since the average pinwheel density is largely preserved during development, it is conceivable that final pinwheel densities can be predicted from densities at an early stage. To test this, we calculated for the set of pinwheel density traces presented in Fig. 6.4 a the cross-correlation $C_{\rho}$ between the set of pinwheel densities $\rho_{j}$ at time $t$ and those at various reference times $t^{\prime}$. As shown in Fig. 6.2 pinwheel densities $\rho_{j}$ were predictable only over a limited time interval. For instance, densities at $t=10^{3}$ were only moderately correlated with the near final densities at $t=10^{4}$. For $t \leq 10^{2}$ pinwheel densities were essentially uncorrelated over the range of one order of magnitude. Compared to the cross-correlation $C$ between maps (Fig. 6.2), pinwheel densities were much less predictable during development This is explained by the fact that the presence or absence of a single active mode can strongly influence the pinwheel density of a map, but has only a relatively small effect on its cross-correlation. Thus, pinwheel densities are not a conserved label of individual maps during development.

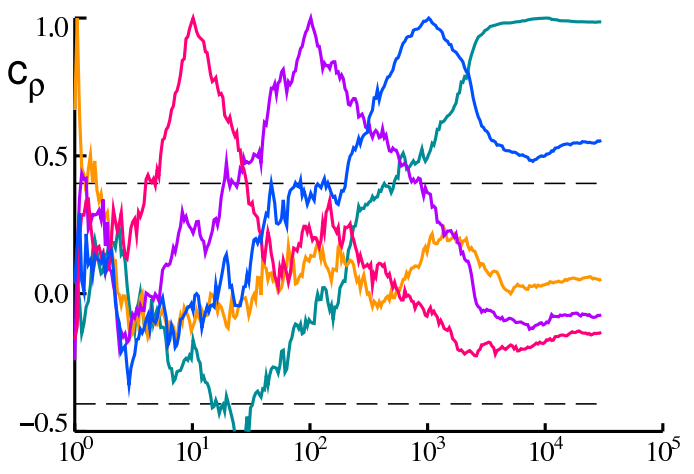

Figure 6.5: Correlations $C_{\rho}$ between pinwheel densities $\rho_{j}$ at time $t$ and at reference times $t=$ $10^{0}$ (orange), $t=10^{1}$ (red), $t=10^{2}$ (violet), $t=$ $10^{3}$ (blue), $t=10^{4}$ (dark green) for the traces from Fig. 6.4 a. Pinwheel densities are generally less predictable compared with the map itself (Fig. 6.2).

\subsubsection{Nearest neighbor statistics}

Having observed that pinwheel densities fluctuate during development, we next asked whether the local statistics of pinwheels is also altered. In particular, we focused on the nearest neighbor distances between pinwheels introduced in Subsection 5.4.2 the distance $h_{++,--}$to the adjacent pinwheel of equal sign, the distance $h_{+-,-+}$to that of opposite sign and $h_{t o t}$, the smallest distance to any other pinwheel. Fig. 6.6a-d shows that the histograms of the three distances remain relatively constant over time (average over $N=10$ solutions obtained for $r=0.3$, 

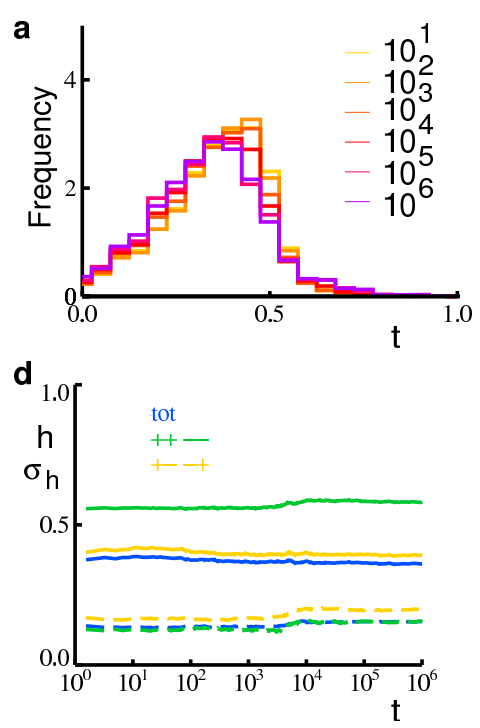
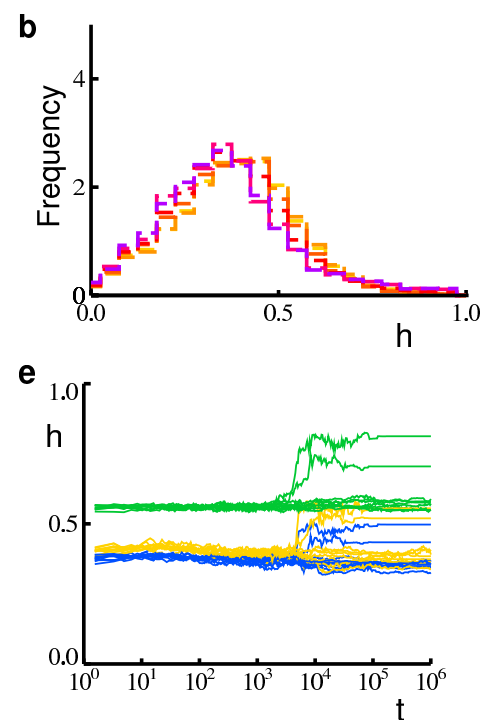

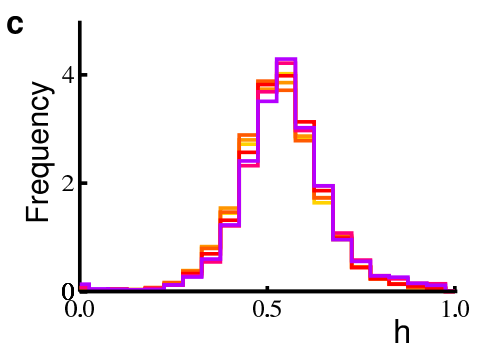

f

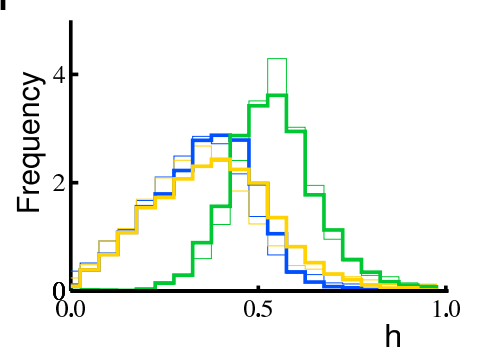

Figure 6.6: Nearest neighbor distances of pinwheels during development. a-c, Histogram of distance to the next pinwheel of arbitrary $($ tot, $\mathbf{a})$, opposite $(+-,-+, \mathbf{b})$, and equal $(++,--$, c) topological charge at different times (normalized, average over the $N=10$ solutions from Fig. 6.4p with $r=0.3$ ). All distributions are preserved over time. d, Average (solid lines) and SD (dashed lines) of the histograms from a-c. Slight changes in mean and SD occur before $t=1 \cdot 10^{4}$, at the time pinwheel densities diverge (compare Fig. 6.4. e, Traces of mean as in d, but for each map individually. Transitions towards larger distances correspond to those leading to lower pinwheel densities. $\mathbf{f}$, Comparison of final histograms (thick lines). Almost all neighbor pinwheels have opposite sign. Planforms (of order $n=20$ ) show very similar distributions (thin lines).

$\sigma=1.7 \Lambda)$. Individual maps that developed into low pinwheel density solutions at $t=t^{*}$ showed transitions towards higher distances at that time (Fig. 6.6e). However, besides these cases the nearest neighbor statistics appeared very stable over time. Fig. 6.6] shows that almost all nearest neighbors were of opposite charge. All histograms were very similar to those for planforms of order $n=20$ (Subsection 5.4.2 indicating that they are largely independent of the two model parameters $r$ and $\sigma$ in the regime $\sigma>1.7 \wedge$. Taken together, this shows that pinwheels are predicted to maintain their nearest neighbor statistics during development.

\subsubsection{Annihilation and creation}

The reorganization of the orientation map during development leads to the annihilation and creation of pinwheels pairs. Both processes are major structural changes 
that alter the topology of the map. The pinwheel annihilation rate defined by

$$
\alpha(t)=\frac{1}{\pi A} \frac{d N_{\alpha}}{d t}
$$

describes the rate of change of the number of annihilated pinwheels $N_{\alpha}$ in a given area $A$. Analogously, the number $N_{\chi}$ of created pinwheels defines

$$
\chi(t)=\frac{1}{\pi A} \frac{d N_{\chi}}{d t}
$$

for the creation rate. To estimate these rates, all annihilation and creation processes must be identified. To achieve this, pinwheels were tracked over time. For details see Methods (Section 7.3).

Both rates were fairly equal throughout development. Fig. 6.7 $\mathrm{a}$, shows averages over the ensembles of solutions from Fig. 6.4a, while Fig. 6.7p shows those from Fig. 6.4 p. The average rates decayed algebraically as

$$
\langle\alpha\rangle,\langle\chi\rangle=b t^{-1}
$$

with different prefactors $b$ before and after the cross-over time $t^{*}=10^{4}$. In the regime of mode competition at $t_{0}<t<t^{*}$ best fits were obtained for $b \approx 1 / 5$, whereas a much smaller value of $b \approx 1 / 20$ was found for the late regime $t^{*}<t<$ $10^{6}$. At the cross-over time both rates decreased abruptly with a slight prevalence of pinwheel creation processes in the beginning followed by a phase of more pronounced annihilation when some of the solutions turn into low pinwheel density configurations (see Fig. 6.2). During the linear phase $t<t_{0}$ annihilation rates $\alpha$ were higher due to the large number of pinwheels present in the initial condition (compare Fig. 6.1).

Given a set of pinwheels present at time $\tau$, what is the fraction $\mu_{\tau}(t)$ of those surviving until $t$ ? This fraction is calculated from

$$
\frac{d \mu_{\tau}}{d t}=-\mu_{\tau} \alpha
$$

with $\mu_{\tau}(\tau)=\mu_{0}$ as initial condition. Inserting the average annihilation rate Eq. 6.7 yields

$$
\mu_{\tau}(t)=\mu_{0}\left(\frac{t}{\tau}\right)^{b}
$$

with exponents $b=1 / 5$ for the early and $b=1 / 20$ for the late regime. Fig. $6.7 \mathrm{~d}$, e shows fractions $\mu_{\tau}(t)$ directly determined from the solutions from Fig. 6.2 by tracking all pinwheels during development present at $t=\tau$. Indeed, pinwheels were found to decay following a power-law with different exponents on both sides of the cross-over time $t=t^{*}$ with average values of $b \approx 0.18$ before and $b=0.03$ after 

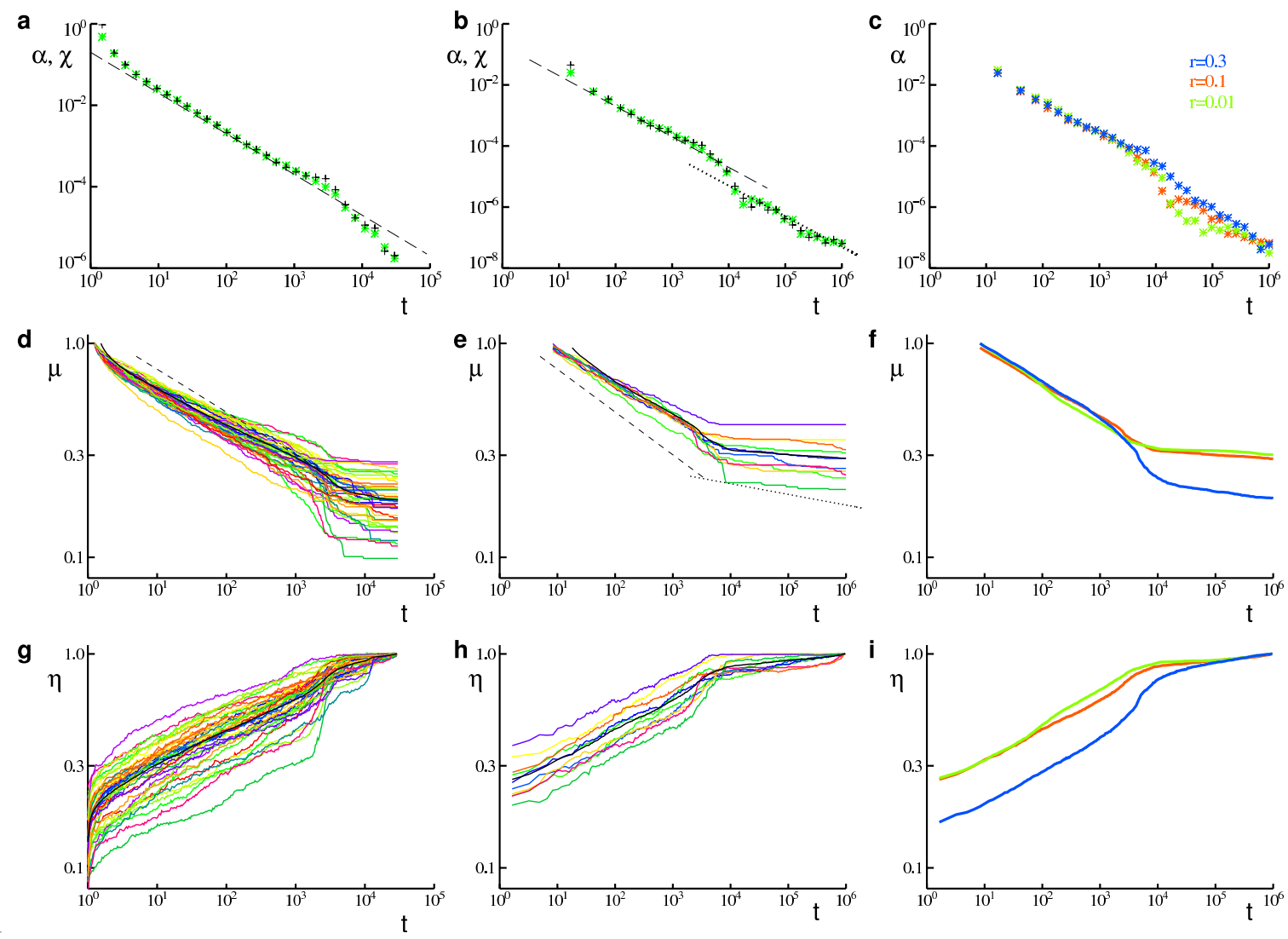

Figure 6.7: Pinwheel annihilation and creation during development. a, Annihilation rate $\alpha$ (black crosses) and creation rate $\chi$ (green stars) normalized and averaged over the $\mathrm{N}=40$ solutions from Fig. 6.4a and, $\mathbf{b}$, for the $\mathrm{N}=10$ simulations from Fig. 6.4p with $r=0.1$ advanced until $t=1 \cdot 10^{6}$. Both rates are nearly equal and decay according to power laws with different prefactors before and after the cross-over time $t^{*}=1 \cdot 10^{4}$. Fits: $\alpha, \chi=b t^{-1}$ with $b=1 / 5$ for $t<t^{*}$, and $b=1 / 20$ for $t>t *$. c. For various $r$ the annihilation rate $\alpha$ is nearly equal for $t<t^{*}$. Subsequently, the rates split up and are higher for larger $r$ due to its faster relaxation of phases $\phi_{j}$. d. Survival fraction $\mu_{\tau}(t)$ of pinwheels present at $\tau=3 \cdot 10^{0}$ for the solutions from a (colors as in 6.4a). The average is presented by the black curve. e, Survival fraction $\mu_{\tau}(t)$ with $\tau=1 \cdot 10^{1}$ for the solutions from $\mathbf{b}$ (colors as in 6.4p). The piecewise algebraic decay $\propto t^{b}$ derives from the annihilation and creation rates $\alpha$ and $\chi$ (see text). Dashed and dotted lines drawn for $b=1 / 5$ and $b=1 / 20$, respectively. $\mathbf{f}$, The survival fraction $\mu_{\tau}(t)$ is independent of $r$ before $t \approx t^{*}$ and, afterwards, becomes smaller for larger $r$. $\mathbf{g}-\mathbf{h}$, The preserved fraction $\eta$ pinwheels at final time $t_{f}=3 \cdot 10^{4}(\mathbf{g}$, for the solutions from $\mathbf{d}$ using identical colors $)$ and $t_{f}=1 \cdot 10^{6}(\mathbf{h}$, from e). i, Preserved fraction $\eta$ for various $r$. All graphs are drawn on logarithmic axes. Note that whereas more processes occur during the early nonlinear phase, they never really come to an end due to algebraically decaying rates. 
$t^{*}$. Across different solutions, the exponent $b$ was relatively similar. The predicted values for $b$ by (6.9) deviated slightly from the measured values since they were based on the average rate $\langle\alpha\rangle$. These results predict that of the pinwheels present at an early stage, a considerable fraction is already annihilated after a short period of development (Fig. 6.7d). Altogether, only $\approx 30 \%$ of the pinwheels survived from $t=t_{0}$ to $t=10^{6}$. The power law decay of rates in the late phase indicates a possibility of structural changes in the map even after very long times.

A related number is the fraction of pinwheels present at time $t$ that survive up to the final time $t_{f}$. This fraction of long term stable pinwheels, called the preserved fraction $\eta$, combines the information in $\mu_{\tau}(t)$ for all values of $\tau$ and $t=t_{f}$. Fig. $6.7 \mathrm{~g}$, h show the fraction $\eta$ for the set of solutions shown in Fig. 6.7 $\mathrm{d}$, e. Again, one finds a power law behavior with different exponents before and after the cross-over point $t=t^{*}$. Consistent with $\mu_{\tau}$, only $\approx 30 \%$ of pinwheels present at the end of the linear phase at $t=t_{0}$ remained until $t=1 \cdot 10^{6}$, but after $t=t^{*}$, most of the pinwheels $(\approx 90 \%)$ were stable up to this very long time.

Up to the cross-over point $t=t^{*}$, these processes were largely independent of the bifurcation parameter $r$. As depicted in Fig. 6.7F, $\mathrm{f}$ and $\mathrm{i}$, the annihilation rate $\alpha$, the creation rate $\chi$, and the surviving fraction $\mu_{\tau}$ were almost identical for $t<3 \cdot 10^{3}$. The same was true for the fraction of long-time stable pinwheels $\eta$ when taking the final time as $t_{f}=3 \cdot 10^{3}$. After this phase for $t>t^{*}$, the creation and annihilation rates depended on $r$. Consistent with a faster phase dynamics, both rates were larger for larger values of $r$ leading to higher rates of change of both fractions $\mu_{\tau}$ and $\eta$ compared to their behavior for small $r$.

\subsubsection{Movement and speed}

Two pinwheels annihilate by moving towards each other, and pinwheels apparently have to repel each other when a new pair is created. Not all movement, however, is related to annihilation or creation processes. We found that pinwheels surviving throughout the whole course of development followed curved trajectories of lengths $\approx \Lambda$. Fig. $6.8 \mathrm{a}, \mathrm{b}$ shows the trajectories through which all pinwheels passed that were present for $1 \cdot 10^{0} \leq t \leq 1 \cdot 10^{6}$. Although the layout of the map changes substantially during this period, pinwheels generally do not reach very far. The distribution of path lengths

$$
l^{j} \approx \sum_{i}\left|\left(\mathbf{x}_{i+1}^{j}-\mathbf{x}_{i}^{j}\right)\right|
$$

calculated from the locations $\mathbf{x}_{i}^{j}$ of pinwheels $j$ in $N=10$ different solutions sampled at times $t_{i}$ was peaked at $\approx 0.9$ column spacings $\Lambda$ with largest values below 2.0^ (Fig. 6.8 , blue curve). Interestingly, there was not a single pinwheel which 

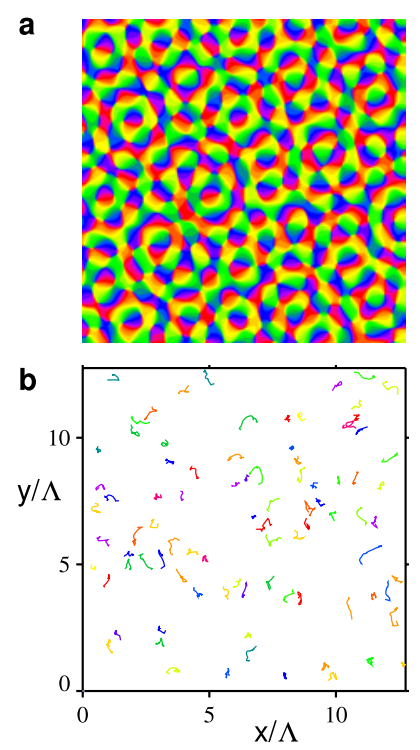
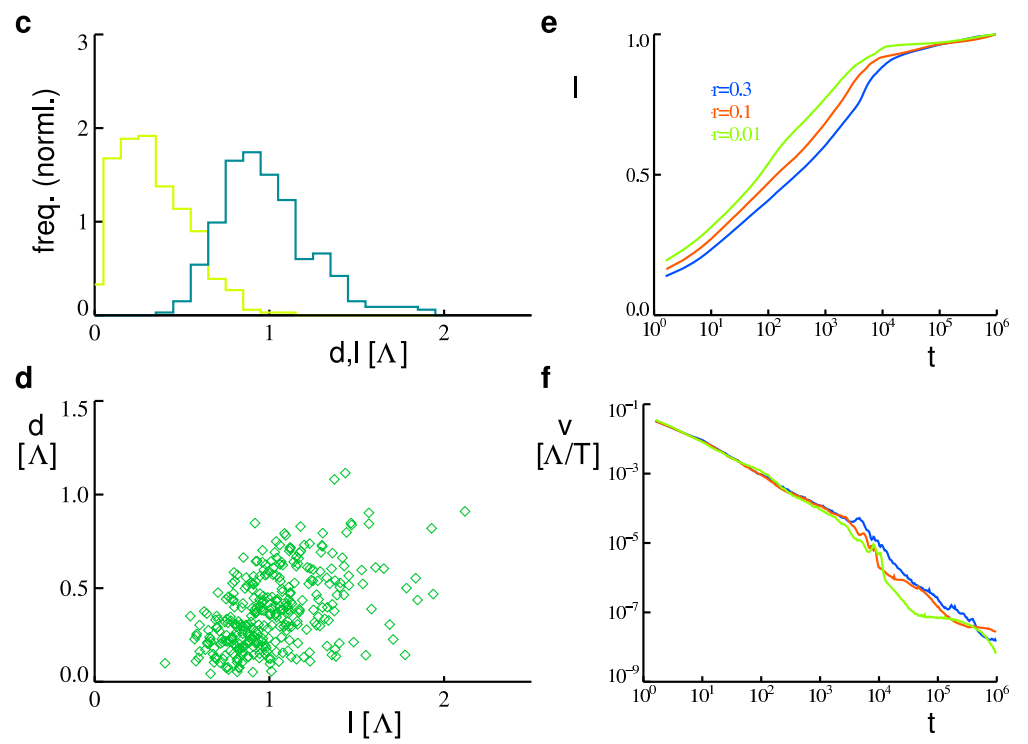

Figure 6.8: Pinwheel movement during development. a, Orientation map at the quasi stationary state at $t=1 \cdot 10^{6}$. $\mathbf{b}$, Trajectories of pinwheels present during $1 \cdot 10^{0} \leq t \leq 1 \cdot 10^{6}$. Different pinwheels $j$ are marked by randomly chosen colors. c, Histograms of final distances $d^{j}$ (yellow) and pathlengths $l^{j}$ (blue) of the $N=10$ solutions from Fig. 6.4p with $r=0.1$. Note that all pinwheels move during development, but not all reach a distant final location. d, Long path lengths $l^{j}$ do not imply large final distances $d^{j}(C=0.48)$. e, $\mathbf{f}$, Average normalized path length $l(\mathbf{e})$ and pinwheel speed $v(\mathbf{f})$ of all pinwheels from $\mathbf{d}(r=0.1)$ and, likewise, for the sets from Fig. 6.4p with $r=0.3$ and $r=0.01$. The pathlength $l$ increases logarithmically with a change of the prefactor at $t=t^{*}$ while the speed $v$ exhibits a discontinuity at this cross-over point. Before this point, the speed of pinwheels is universal for various $r$.

did not show any movement. Final distances

$$
d^{j}=\left|\left(\mathbf{x}_{\text {final }}^{j}-\mathbf{x}_{0}^{j}\right)\right|
$$

of pinwheels from their initial locations were, in contrast, often close to zero (yellow curve). Pinwheels did not terminate further than $1 \wedge$ and typically with a distance of $0.25 \Lambda$. Pinwheels moving further away from their initial location often curved around or changed directions. Pinwheel distances $d^{j}$ and path lengths $l^{j}$ were only weakly correlated (Fig. 6.8 d). Long path lengths $l^{j}$ not always resulted in remote final locations.

Pinwheels moved predominantly during the early stage of development. After the cross-over time $t=t^{*}$, much shorter routes were passed. This was quantified by the average fractional path length

$$
l(t)=\left\langle l^{j}(t) / l_{\text {total }}^{j}\right\rangle_{j},
$$


where the average is over the pathlengths $l^{j}(t)$ of individual pinwheels $j$, each normalized by its total pathlength $l_{\text {total }}^{j}=l^{j}\left(t_{f}\right)$. This quantity is shown in Fig. 6.8 e for different values of $r$ calculated for the solutions from Fig. 6.4p. The relative path length increased approximately logarithmically from the initial condition $l_{0}=l\left(t_{0}\right)$ as

$$
l=l_{0}+a \ln \left(t / t_{0}\right)
$$

with different prefactors $a$ before and after the cross-over phase and (Fig. 6.8p). For all $r$, the largest fraction of the total path $(\approx 90 \%)$ was covered for $t<t^{*}$ due to the relative large value of $a \approx 0.1$ in this regime. The instantaneous mean speed of pinwheels calculated by

$$
v(t) \approx v\left(t_{i}\right)=\frac{\left\langle\left|\left(\mathbf{x}_{i+1}^{j}-\mathbf{x}_{i}^{j}\right)\right|\right\rangle_{j}}{t_{i+1}-t_{i}}
$$

was found to decay as

$$
v \sim a t^{-1}
$$

which was consistent with its interpretation as the derivative $v=d\left\langle l^{j}\right\rangle / d t$ of the average path length with respect to time. The cross-over phase around $t \approx t^{*}$ was noticeable from a discontinuity of pinwheel speed $v$ corresponding to a kink in the relative path length $l$. After this phase, the speed of pinwheels dropped to slower values with much smaller prefactors. Whereas in the late phase for $t>$ $t *$, the speed of pinwheels depended on $r$, it was universal before the cross-over phase. After this time, the map develops further albeit with a much smaller rate presumably determined by the relaxation of phases.

\subsection{Temporal scaling of map dynamics}

The proceeding subsections have shown that the behavior of pinwheels during development is in many aspects independent of the bifurcation parameter $r$ during the early phase of nonlinear competition of modes. This defines a temporal scale of $t^{*} \approx 1 \cdot 10^{4}$ on which the development takes place. What is the origin for this scale? in the following we analyze whether it corresponds to the decay rate of an unstable fix point. Initially, at the end of the phase of linear growth at $t \approx 1 \cdot 10^{1}$ many modes are active. During the consecutive phase of nonlinear competition the final set of active modes are selected. In general, one expects the dynamics to wander around hyperbolic fix points in phase space. At each fix point the the largest eigenvalue of the linearized dynamics determines the decay rate. The absolute time for the attractor to settle into a stationary configuration of modes is mainly determined by the unstable fix point approached with smallest decay rate. 


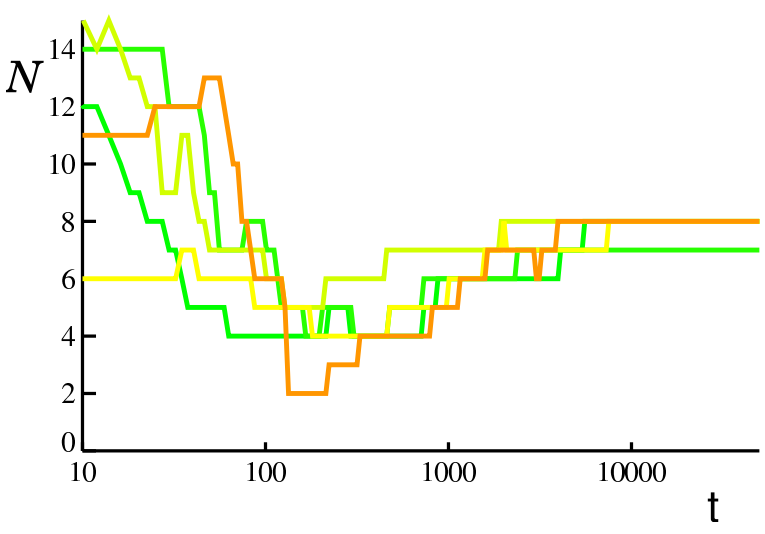

Figure 6.9: Unstable fixpoints encountered during development. Traces of number of active modes $n$ for $N=5$ solutions form Fig. 6.4p calculated with $r=0.01$. Modes were counted by selecting all localized zones in the powers spectrum $|\tilde{z}(\mathbf{k})|^{2}$ larger than 0.6 times the maximum. Stationary solutions consist of $n=8$ active modes in four cases and $n=7$ in one case. At the end of the linear phase at $t \approx 1 \cdot 10^{1}$ most opposite modes are active. Transiently, most modes are suppressed.

What are the unstable fixpoints encountered in the course of development? Fig. 6.9 shows for solutions from Fig. 6.4p the traces of the number $\mathcal{N}$ of modes active at time $t$. The number of finally active modes was $n=8$ or $n=7$. At the beginning of the phase of nonlinear competition among modes at $t \approx 10^{1}$ almost twice as many modes were active including many pairs of opposite modes. Consecutively, the number of modes decayed rapidly leading to a suppression of most modes at $t \approx 3 \cdot 10^{2}$. At even later stages individual modes get reactivated until the final number of a stable configuration is obtained. The selection terminated at $t^{*} \approx 10^{4}$ consistent with previous characterization of the cross-over phase between amplitude and phase dynamics at that time. The time constant of the decay is of the order of $T \sim 10$ and much faster than that of the reactivation being of order $T \sim 100-1000$.

The competition among modes is described approximately by the amplitude formalism. In the following, I consider planforms of order $n$ written as

$$
z(\mathbf{x})=\sum_{j} A_{j} e^{i l_{j} \mathbf{k}_{j} \mathbf{x}}+\sum_{j} B_{j} e^{-i l_{j} \mathbf{k}_{j} \mathbf{x}}
$$

where $\mathbf{k}_{j}=k_{c}(\cos (j \pi / n), \sin (j \pi / n))$ with $j=1,2, \ldots, n$ and the amplitudes are given by e.g. $A_{j}=\mathcal{A}_{j} e^{i \phi_{\mathcal{A}_{j}}}$. The amplitude equations take the form

$$
\begin{aligned}
\frac{\partial}{\partial t} A_{i} & =r A_{i}-\sum_{j} g_{i j}\left|A_{j}\right|^{2} A_{i}-\sum_{j}\left(1+\delta_{i j}\right) g_{i j}\left|B_{j}\right|^{2} A_{i}-2 \sum_{j} f_{i j} A_{j} B_{j} \bar{B}_{i} \\
\frac{\partial}{\partial t} B_{i} & =r B_{i}-\sum_{j} g_{i j}\left|B_{j}\right|^{2} B_{i}-\sum_{j}\left(1+\delta_{i j}\right) g_{i j}\left|A_{j}\right|^{2} B_{i}-2 \sum_{j} f_{i j} A_{j} B_{j} \bar{A}_{i}
\end{aligned}
$$

independent of the configuration $\left(l_{1}, l_{2}, \ldots, l_{n}\right)$. For $\sigma>\Lambda$, the interaction coeffi- 
cients are well approximated by

$$
\begin{array}{ll}
g_{i i}=1, & g_{i i^{-}}=2, \quad g_{i j}=g \\
f_{i i}=0, & f_{i i^{-}}=0,
\end{array}
$$

with $j \neq i, i^{-}$. The amplitude equations 6.17 have solutions with uniform absolute values

$$
\begin{aligned}
\mathcal{A}_{i} & =\mathcal{A}=\sqrt{\frac{r}{\sum_{j} g_{i j}}} \\
\mathcal{B}_{i} & =0
\end{aligned}
$$

and arbitrary phases $\phi_{\mathcal{A} i}$ which are stable for $0<g<1$.

To analyze the stability of fixpoints it is convenient to split the amplitudes equations (6.17) into equations for the absolute values $\mathcal{A}_{i}$ and the phase $\phi_{\mathcal{A} i}$. Performing the derivative at the left hand side of Eq. (6.17) and separating the real and imaginary part yields

$$
\begin{aligned}
\frac{\partial}{\partial t} \mathcal{A}_{i}= & r \mathcal{A}_{i}-\sum_{j} g_{i j} \mathcal{A}_{j}^{2} \mathcal{A}_{i}-\sum_{j}\left(1+\delta_{i j}\right) g_{i j} \mathcal{B}_{j}^{2} \mathcal{A}_{i}- \\
& 2 \sum_{j} f_{i j} \mathcal{A}_{j} \mathcal{B}_{j} \mathcal{B}_{i} \cos \left(\left(\phi_{\mathcal{A} j}+\phi_{\mathcal{B} j}\right)-\left(\phi_{\mathcal{A} i}+\phi_{\mathcal{B} i}\right)\right) \\
\frac{\partial}{\partial t} \phi_{\mathcal{A} i}= & -2 \sum_{j} f_{i j} \frac{\mathcal{A}_{j} \mathcal{B}_{j} \mathcal{B}_{i}}{\mathcal{A}_{i}} \sin \left(\left(\phi_{\mathcal{A} j}+\phi_{\mathcal{B} j}\right)-\left(\phi_{\mathcal{A} i}+\phi_{\mathcal{B} i}\right)\right)
\end{aligned}
$$

and a corresponding expression for $B_{i}$. It can be easily seen that the linearized equations for the dynamics of the moduli $\mathcal{A}_{j}$ and phases $\phi_{\mathcal{A} j}$ are decoupled in the vicinity of a stationary solution. Hence, it is possible to discuss the stability of stationary solutions with respect to phase perturbations and amplitude perturbations separately. A stationary configuration of the absolute values of the amplitudes $\mathcal{A}_{j}, \mathcal{B}_{j}$ fulfills

$$
\begin{aligned}
0 & =r \mathcal{A}_{i}-\sum_{j} g_{i j} \mathcal{A}_{j}^{2} \mathcal{A}_{i}-\sum_{j}\left(1+\delta_{i j}\right) g_{i j} \mathcal{B}_{j}^{2} \mathcal{A}_{i}-2 \sum_{j} f_{i j} \mathcal{A}_{j} \mathcal{B}_{j} \mathcal{B}_{i} \\
0 & =r \mathcal{B}_{i}-\sum_{j} g_{i j} \mathcal{B}_{j}^{2} \mathcal{B}_{i}-\sum_{j}\left(1+\delta_{i j}\right) g_{i j} \mathcal{A}_{j}^{2} \mathcal{B}_{i}-2 \sum_{j} f_{i j} \mathcal{B}_{j} \mathcal{A}_{j} \mathcal{A}_{i}
\end{aligned}
$$

when the phases satisfy $\phi_{\mathcal{A} j}+\phi_{\mathcal{B} j}=\Phi_{0}=$ const. .

To first analyze the initial phase of decay, we consider the unstable fixpoint of Eq. (6.17) of a planform with all $2 n$ modes active. All modes $A_{i}$ and $B_{i}$ have the same modulus 


$$
\begin{aligned}
\mathcal{A}_{i} & =\mathcal{A} \\
\mathcal{B}_{i} & =\mathcal{A}
\end{aligned}
$$

that is given through (6.21) by

$$
\mathcal{A}=\sqrt{\frac{r}{2 \sum_{j} g_{i j}+g_{i i}+2 \sum_{j} f_{i j}}} .
$$

The linearized equations for perturbations $a_{i}=\mathcal{A}_{i}-\mathcal{A}$ around the unstable fixpoint Eq. (6.22) are

$$
\begin{aligned}
\frac{\partial}{\partial t} a_{i}= & r a_{i}-\mathcal{A}^{2} \sum_{j}\left(2+\delta_{i j}\right) g_{i j} a_{i}-2 \mathcal{A}^{2} \sum_{j}\left(g_{i j}+f_{i j}\right) a_{j}- \\
& A^{2} \sum_{j} f_{i j} b_{i}-2 \mathcal{A}^{2} \sum_{j}\left(\left(1+\delta_{i j}\right) g_{i j}+f_{i j}\right) b_{j} \\
= & \sum_{j}\left(D_{i j} a_{j}+D_{i j+n} b_{j}\right)
\end{aligned}
$$

together with the symmetric expressions for $b_{i}=\mathcal{B}_{i}-\mathcal{A}$. The matrix $\mathbf{D}=\left(D_{q p}\right)$ is symmetric and has therefore $2 n$ real eigenvalues $w_{i}$. It is easy to see that there are $n$ eigenvectors $\mathbf{v}_{i}$ of the form

$$
\begin{aligned}
a_{i} & =-b_{i}=1 \\
a_{j \neq i} & =b_{j \neq i}=0
\end{aligned}
$$

with eigenvalue

$$
\begin{aligned}
w_{e} & =r-\mathcal{A}^{2}\left(2 \sum_{j} g_{i j}-g_{i i}-2 \sum_{j} f_{i j}\right) \\
& =r-\frac{2 \sum_{j} g_{i j}-g_{i i}-2 \sum_{j} f_{i j}}{2 \sum_{j} g_{i j}+g_{i i}+2 \sum_{j} f_{i j}} \\
& =\frac{2}{3} r .
\end{aligned}
$$

describing the decay from the fixpoint $(6.22)$ due to the competition between antipairs of modes. Following (6.26), it exhibits a similar time scale as the exponential growth during the initial linear phase of the dynamics (Fig. 5.1).

A further analysis shows that these eigenvectors comprise all instable directions. Another eigenvalue, $n-1$ times degenerated, is given by

$$
w_{0}=\frac{6 g-6}{3(n-1) g+3} r
$$


which is always negative for $0<g<1$. The last eigenvalue

$$
w_{1}=-2 r
$$

corresponds to a uniform perturbation

$$
a_{i}=b_{i}=1 .
$$

Thus, a transient that is close to fixpoint $(6.22)$ in the early nonlinear phase experiences on temporal scale

$$
T_{e}=\frac{1}{w_{e}}=\frac{3}{2} \frac{1}{r}
$$

the decay of one pair of modes. This is about one order of magnitude smaller than the observed time scale of $T \sim 10$ for the decay of amplitudes in Fig. 6.9 The form of the decrease of the number of active amplitudes in Fig. 6.9 suggests that it arises from a cascade of fixpoints with increasing temporal scales encountered during early evolution. However, since we are primarily interested in explaining the occurrence of the scale $t^{*}$, we do not further pursue this here.

At the later stage of nonlinear mode competition, transient states are characterized by the absence of one or more modes compared to the final configuration. As shown in Fig. 6.9, states comprising an increasing number of modes may be visited during development. First, an unstable fixpoint

$$
\begin{aligned}
\mathcal{A}_{i} & =\mathcal{A}, \quad i \neq l \\
\mathcal{A}_{l} & =0 \\
\mathcal{B}_{i} & =0
\end{aligned}
$$

is considered in which only a single mode $\mathcal{A}_{l}$ is inactive compared to the stable solution (6.19). The uniform amplitude $\mathcal{A}$ is determined from (6.21) which simplifies to

$$
0=r \mathcal{A}_{i}-\sum_{j \neq l} g_{i j} \mathcal{A}_{j}^{2} \mathcal{A}_{i}, \quad i \neq l
$$

yielding

$$
\mathcal{A}=\sqrt{\frac{r}{\sum_{j \neq l} g_{i \neq l}}} .
$$

The linearized equations around solution 6.31) become

$$
\begin{aligned}
\frac{\partial}{\partial t} a_{i} & =r a_{i}-\mathcal{A}^{2} \sum_{j \neq l} g_{i j} a_{i}-\delta_{l i} 2 \mathcal{A}^{2} \sum_{j \neq l} g_{i j} a_{J} \\
\frac{\partial}{\partial t} b_{i} & =r b_{i}-\mathcal{A}^{2} \sum_{j \neq l}\left(1+\delta_{i j}\right) g_{i j} a_{i}-\delta_{l i} 2 \mathcal{A}^{2} \sum_{j \neq l} f_{i j} a_{j} .
\end{aligned}
$$


The eigenvector of greatest interest is

$$
\begin{aligned}
& a_{l}=1 \\
& a_{i}=0, \quad i \neq l \\
& b_{i}=0
\end{aligned}
$$

pointing directly towards the attractor (6.19). Its eigenvalue is

$$
\begin{aligned}
w & =r-\frac{\sum_{j \neq l} g_{l j}}{\sum_{j \neq l} g_{i \neq l j}} \\
& =r(1-g) \frac{1}{(n-2) g+1} \\
& \approx r \epsilon /(n-1)
\end{aligned}
$$

where $\epsilon=1-g$ and where the last step is valid for $\epsilon \ll 1$. Thus, for $g$ close to 1 the growth rate of the last missing mode depends linearly on $\epsilon$ and becomes arbitrary small for $g \rightarrow 1$. The mechanism for this behavior is that the auto and cross interaction is almost identical as apparent from the fraction in the first step of (6.36). By symmetry, Eq. (6.34) has another eigenvector with identical eigenvalue, namely the one expressing a growth of $\mathcal{B}_{1}$ instead of $\mathcal{A}_{1}$. All further eigenvalues are always negative. With the parameters used in Fig. 6.9 $n=8$ and $g=0.98$, the time scale of growth of this mode is $T_{l}=350 / r$ in approximate agreement with the observed scale.

As indicated in Fig. 6.9 the stable state may also be reached via fixpoints with more than one mode missing. In fact, there exist unstable fixpoints with $n-k$ active modes of the form

$$
\begin{aligned}
\mathcal{A}_{i} & =\mathcal{A}, \quad i \notin\{1,2, \ldots k\} \\
\mathcal{A}_{1} & =\mathcal{A}_{2}=\cdots=\mathcal{A}_{k}=0 \\
\mathcal{B}_{i} & =0
\end{aligned}
$$

and with amplitudes

$$
\mathcal{A}=\sqrt{\frac{r}{\sum_{j=k+1}^{n} g_{i \neq 1, \ldots, k j}}} .
$$

There are $2 k$ eigenvectors each of them corresponding to the growth of one of the possibly growing modes $\mathcal{A}_{j \leq k}$ or $\mathcal{B}_{j \leq k}$, respectively. They have degenerated eigenvalues

$$
\begin{aligned}
w_{l} & =r(1-g) \frac{1}{(n-1-k) g+1} \\
& \approx r \epsilon \frac{1}{n-k}
\end{aligned}
$$



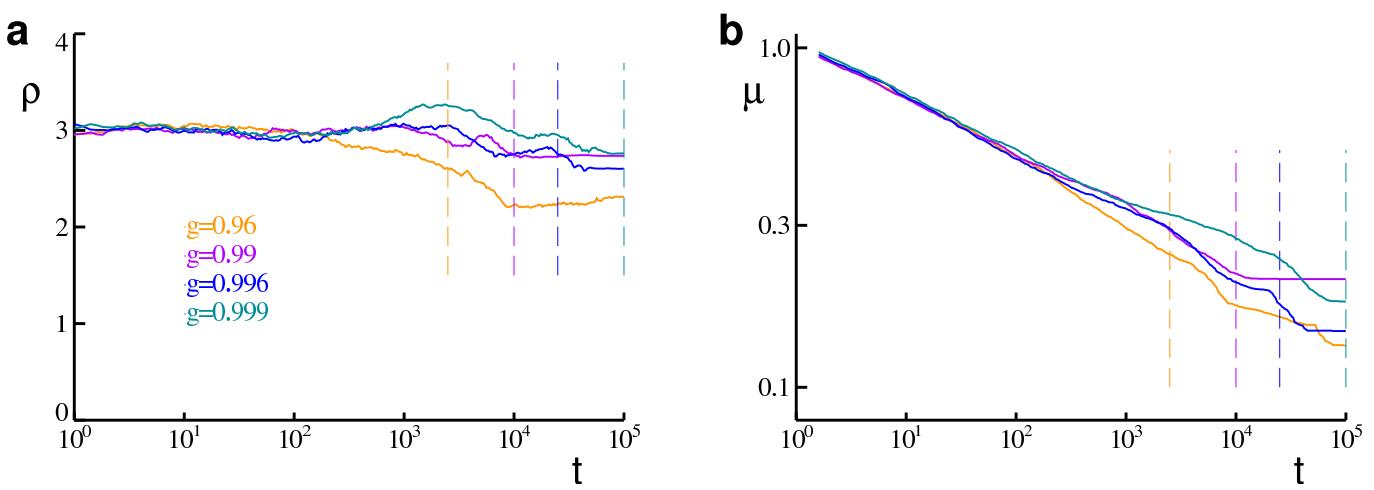

Figure 6.10: Time scale of mode competition depends on $g$. a, Traces of average pinwheel densities $\langle\rho\rangle$ for various $g$ (parameter as in Fig. 6.1 $N=5$ solutions per $g$ ). Dashed vertical lines show the scaling predicted (Eq. 6.36) for the end of the cross-over phase at time $t^{*}$. Estimating $t_{g}^{*}=1 \cdot 10^{5}$ for $g=0.999$, other $t_{g^{\prime}}^{*}$ were rescaled by $t_{g^{\prime}}^{*}=t_{g}^{*}(1-g) /\left(1-g^{\prime}\right)$. $\mathbf{b}$, The fraction $\langle\mu\rangle$ of surviving pinwheels for the same set of solutions. Note that the slope of $\langle\mu\rangle$ shows a tendency to be steeper for smaller $g$ indicating a faster dynamics. However, the actual values of $t_{g}^{*}$ are difficult to estimate from the curves.

which decrease with the number of yet active modes $n-k$. Thus, in the late phase of nonlinear mode competition lacking modes grow on a temporal scale

$$
T_{l}=\frac{1}{w_{l}}=\frac{n-k}{\epsilon} \frac{1}{r} .
$$

Inserting the parameters from Fig. 6.9 yields time scales ranging between $T_{l}=50 / r$ for one mode active and $T_{l}=350 / r$ for seven modes active. This time scale scales with $1 / r$ explaining the observed scaling of the cross-over point $t=t^{*}$ in the full dynamics. Compared to Fig. 6.9 the time scale $T_{l}$ appears to be a factor of $2-3$ smaller. However, if for a given solution only a subset of possible modes grow around a fixpoint Eq. 6.37, it is forced to visit another fixpoint of type Eq. (6.37) with reduced $k$. This may explain why the scale of mode growth seems to increase between $t \approx 10^{2}$ and $t \approx 10^{3}$. For instance, with $k \approx 5$ the effective scale would be $T_{l} \approx 10^{3} / r$ which is consistent with the observed final time of the cross-over phase is $t^{*} \approx 1 \cdot 10^{4}$.

The preceding analysis showed that the temporal scale on which the final mode configuration - the primary determinant of the pinwheel density - forms is determined by the parameter $g$ controlling the relative strength of the local and non-local nonlinear interaction. Following Eq. 6.36) and Eq. (6.39), the cross-over time $t^{*}$ is predicted to scale $\sim \epsilon$. In order to test this, pinwheel dynamics have been analyzed in solutions calculated for various values of $g$. As shown in Fig. 6.10 a for the examples of the average pinwheel density $\langle\rho\rangle$, qualitatively, this scaling is reproduced, especially for larger $g$ as can be seen from the point of near stationarity of 
the pinwheel density. However, it was difficult to identify the cross-over time with higher precision than about half an order of magnitude rendering a comparison for the different values of $g$ difficult. This difficulty arises from the large variation of individual pinwheel density traces (Fig. 6.4) and may be circumvented by using larger ensembles (but compare also, e.g., Fig. 6.8 where the ensemble was twice as large). The reason for this variation is unclear at present. One possible explanation is that solutions contain different grains (which is generally expected for large $r$ ) and that the pinwheel density fluctuation is influenced by the movement of grain boundaries during development. As shown in Fig. 6.10p, the average surviving fraction $\langle\mu\rangle$ provided a somewhat better indication for the different time scales. The slope of $\langle\mu\rangle$ was steeper for smaller $g$ which is consistent with a faster dynamics to be expected. Thus, at least on a qualitative level, the results of the analysis were reproduced by the full dynamics. The cross-over time $t^{*}$ until which the reorganization is relatively strong depends through the parameter $g$ on the relative strength of nonlocal interactions. The weaker these interactions compared to the purely local contribution, the faster near final pinwheel densities emerge.

\subsection{Pinwheel densities in a bounded area}

The presence of boundaries that confine the system can modify the pattern selection process. Solutions must match the boundary conditions such that among the set of possible solutions of the free equations only a subset might be favored with lateral boundary conditions. Furthermore, constraints along the boundary might alter the structure of the solution in its vicinity. The range of direct influence is typically of $\mathcal{O}(\sqrt{r})$ and can thus exceed $\Lambda$. For orientation maps in the visual cortex estimated aspect ratios are $\Gamma \approx 25$ for the cat [44], galago [73] and tree shrew [13]. The area of the map under direct influence of the boundary constraint might be a considerable part of the map. Thus, both mechanisms can alter the layout of the selected map significantly which can influence it statistics such as the pinwheel densities. In the tree shrew, for instance, the layout of the pattern along the border to visual cortical area V2 often consists of stripes oriented perpendicular to the border (see Fig. 2.1 or Fig. 4.1) resulting in a pinwheel density systematically lower than average in this region as shown in Section 4.4 .

While an effect of the boundary on the development of orientation preference along the boundary is plausible, its precise nature is difficult to specify at present. Presumably, both the long-range and short range intracortical connections are organized differently for boundary neurons. long-range connections are shown in tracing studies to range over several columns spacings $\Lambda$ in all cortical directions [2] 13 [55]. However, for neurons at the boundary of the horizontal peripheral representation connections can only be formed in one direction. In the border region representing the vertical meridian, instead, neurons are known to exhibit exten- 
sive connections with the contralateral V1 representing the other visual hemifield. Similarly, orientation columns in the bulk presumably experience different input from nearby columns through short range connections than columns located at the border.

An alternative approach followed here is to judge the nature of the boundary conditions by the columnar layout observed in experiments. In the tree shrew columns form stripe-like domains along the V1 border towards the adjacent area V2 (Fig. ). In cat and ferret V1 this tendency is less pronounced. However, in the peripheral representation of cat V1, columns are observed to form stripe-like domains perpendicular to the boundary [44]. Therfore, boundary conditions might be chosen

$$
\begin{aligned}
z(\mathbf{x}) & =0 \\
\nabla_{b} \cdot z(\mathbf{x}) & =0
\end{aligned}
$$

where $\nabla_{b}$ is the derivative perpendicular to the boundary. These boundary conditions have been investigated in various systems [23]. For systems with ideally stripe-like solutions (e.g. for the classical Swift-Hohenberg equation) they have been shown to enforce the rolls to be oriented perpendicular to lateral wall. For the quasiperiodic solutions investigated here, this is less clear. Since stripe like regions are pinwheel sparse the pinwheel density will be reduced near the boundary. As observed for the tree shrew (Fig. 4.11), the pinwheel density in the bulk region could be larger and compensate for this. Indeed, for topological reasons, perpendicular stripes along the entire boundary induces additional pinwheels of number $L / \Lambda$ where $L$ is the length of the boundary Fig. a. However, both contributions do not scale equally and are expected to depend on $r$. Since the shortest influences of boundaries and thus the largest overall pinwheel densities are expected for large $r$, solutions for a relatively large value of $r=0.3$ have been sought.

A representative example is shown in Fig. 6.11. Already at $t=1 \cdot 10^{2}$ the pattern segregated into a pinwheel rich bulk part and a pinwheel sparse boundary part consisting of band-like orientation columns arranged perpendicular to the border. At successive times, the pattern in the bulk region reorganized and after $t=1 \cdot 10^{6}$ a pattern reminiscent to the patterns obtained with periodic boundary conditions (Fig. 6.1) appeared. The layout along the boundary resembles the layouts observed in tree shrew V1 along the border towards area V2 (Fig. 2.1).

The pinwheel density $\rho(t)$ calculated for $N=3$ different initial conditions is shown in Fig. 6.12a. In contrast to the case of periodic boundary conditions (Fig. 6.4 immediately after the linear phase at $t \approx 10^{1}$ pinwheel densities decreased to reach a plateau of $\rho \approx 2.4$ between $10^{2} \leq t \leq 10^{4}$. The decrease is explained by the formation of the stripe like organization along the boundary starting already before $10^{2}$ as indicated in Fig. 6.11. During the plateau, values from different solutions were very similar. The cross-over time $t^{*} \approx 10^{5}$ was consistent with the prediction 

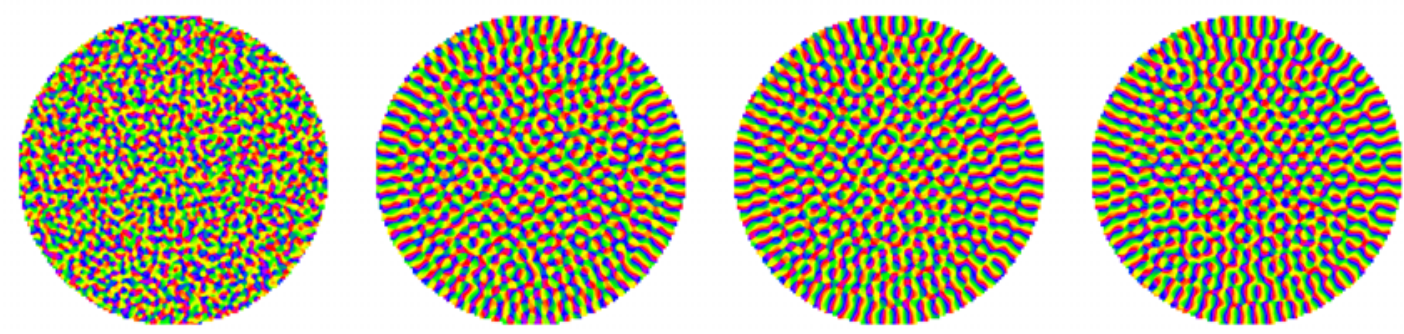

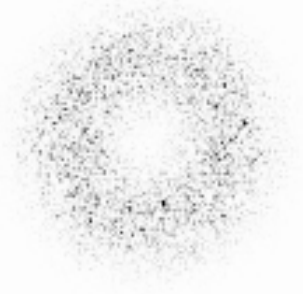

$t=0$

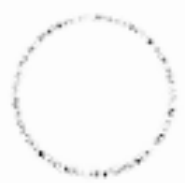

$t=1 \times 10^{2}$

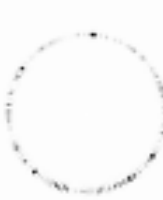

$t=1 \times 10^{4}$

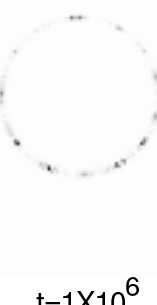

Figure 6.11: Development of orientation map in a bounded area. Eq. (5.1) was integrated on a disk with boundary conditions 6.41) (aspect ratio $\Gamma=23.5, r=0.3, g=0.999$, and $\sigma=1.7$ ). The upper row shows the initial state (band-pass filtered Gaussian random field, left), two intermediate states (meddle) and the near stationary state (right). The lower row shows the power spectrum of each state. Note that the bulk of the final state resembles solutions obtained with periodic boundary conditions (compare Fig. 6.1) while along the border, stripe-like pinwheel sparse domains organize perpendicular to the boundary resembling layouts observed in the tree shrew V1 along the V1/V2 border (Fig. 4.1).

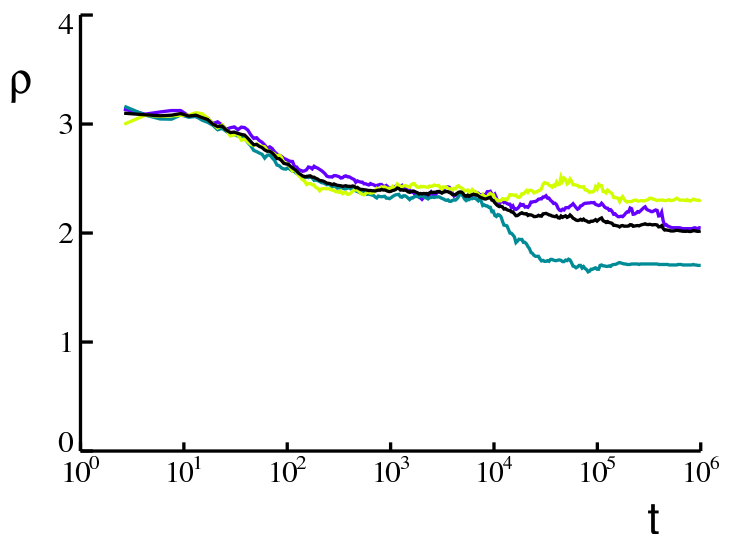

Figure 6.12: Development of pinwheel densities in a confined circular area. Traces of pinwheel density $\rho(t)$ for $N=3$ different initial conditions. Parameters as in Fig. 6.11. Densities start decaying immediately after the end of the linear phase at $t \approx 10^{1}$. Final values at $t=10^{6}$ cover a range $1.3 \leq \rho \leq 2.6$ with an average around $\rho=2$ which is considerably smaller than for periodic boundary conditions (compare Fig. 6.4). The cross-over to pure phase dynamics occurred around $t^{*} \approx 10^{5}$ as predicted from 6.40 with $g=0.999$.

6.10 from the amplitude equations for $g=0.999$ and similar to the case of periodic boundary conditions when using the same value of $g$ (Fig. 6.10). Before $t^{*}$ the traces started to diverge indicating the development of planform like solutions in the bulk of the pattern. Indeed, the final solution depicted in Fig. 6.11 consisted 
a

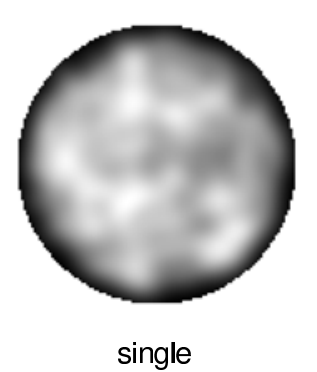

b

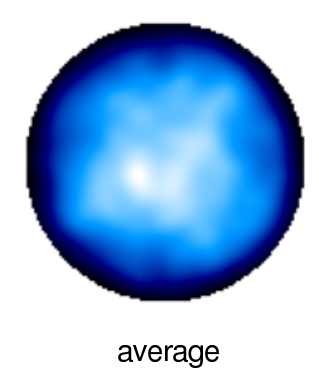

c

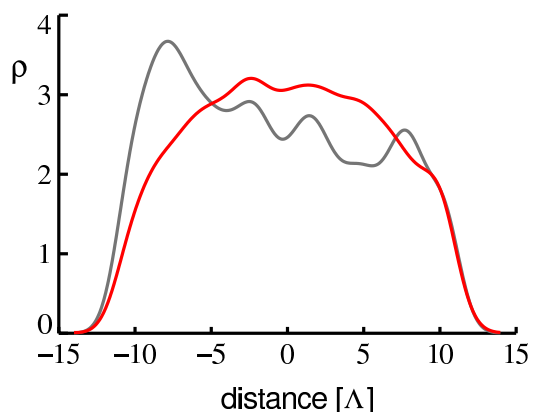

Figure 6.13: Systematic inhomogeneity of local pinwheel density induced by boundary. a, Local pinwheel density of the final state shown in Fig. 6.11 b, Average local pinwheel density in the final states calculated from 10 different initial conditions (compare Fig. 6.12). c, Horizontal (red) and vertical (blue) cross section through the average local density from $\mathbf{b}$. In the bulk region the pinwheel density $\rho$ is close to the number $\pi$ while along the border it decays to zero.

of discrete modes distributed around the critical circle. The pinwheel densities $\rho$ of the final solutions exhibited a similar distribution as for the periodic boundary conditions besides a negative shift of $\approx 0.6$. Thus, the effect of boundary conditions 6.41) are a reduction of pinwheel densities towards unrealistic small values.

Fig. 6.13 shows that this reduction of pinwheel density is mainly due to the border regions. The local pinwheel density $\rho(\mathbf{x})$ (defined as in Section ) of a map advanced until $10^{5}$ shown in Fig. 6.13a and the systematic variation, i.e. the average $\langle\rho(\mathbf{x})\rangle$ over $N=10$ maps of local pinwheel density depicted in Fig. 6.13b, revealed a strong reductions of local pinwheel density along the border reflecting the stripe-like organization of columns. Cross sections through the center of $\rho(\mathbf{x})$ and $\langle\rho(\mathbf{x})\rangle$ are shown in Fig. 6.13. While in the bulk region $\langle\rho(\mathbf{x})\rangle$ is close to the number $\pi$, the reductive influence of the border is apparent over a distance of more than 5 column spacings $\Lambda$. Thus compared to the tree shrew where a relatively high pinwheel density in the bulk of the pattern balanced the regions of low density along the border, this was not the case in the model. Moreover, whereas in the tree shrew, pinwheel densities along boundaries were only moderately reduced to values around $\rho \approx 2$, this reduction was much stronger to $\rho \approx 0$ near the boundary. This suggests that the boundary condition (6.41) may be improper for describing the influence of the boundary on the development of the orientation map.

\subsection{Discussion}

The investigation of the evolution of the orientation map during development revealed a number of interesting effects useful as a guideline for upcoming ex- 
periments. Statistically, the layout of the orientation map was, apart from the early phase, remarkably stable during development. The average pinwheel density and distributions of pinwheel nearest neighbor distances were largely preserved. Quantities that more sensitively reflected the changes during development were proposed and analyzed including pinwheel annihilation and creation rates, pinwheel pathlengths and pinwheel speed.

Consistent with all analyzed quantities, we identified two subsequent phases of map development. During the first phase of nonlinear competition among active modes, the development was found to be largely independent of the bifurcation parameter $r$. The duration of this phase, denoted as the cross-over time $t^{*}$, was equal on a timescale rescaled by $r$ as confirmed analytically by a weakly nonlinear analysis. The late phase was primarily determined by the relaxation of the phases of the finally active modes depended on $r$.

Pinwheel densities from individual maps were found to fluctuate around $\rho \approx \pi$ during the first phase, while in the late phase, a fraction of low density maps was observed associated with the low density states revealed by weakly nonlinear analysis [105] (Fig. 2.5). The cross-over from high to low density states often occurred relatively abrupt. Consistent with a preserved average pinwheel density, we found equal annihilation and creation rates for pinwheel during development. Both rates decayed algebraically with an equal exponent but different prefactors before and after the cross-over time $t^{*}$. Consequently, also the survival fraction decayed as a power law with different exponents before and after $t^{*}$ while the preserved fraction showed an approximately algebraic increase. Individual pinwheels were found to move on curved paths with speed decaying as $\sim t^{-1}$. Consistently, the pathlengths increased approximately logarithmically with different prefactors before and after the cross-over time $t^{*}$ such that about $90 \%$ of the total pathlength of $\approx \wedge$ were covered before $t^{*}$. Thus, on the one hand, the strength of reorganization of the orientation map decreases algebraically during development. Consequently, the largest changes occur during the earliest phase of development, and the map appears rather stable throughout later stages although changes do not really terminate even after very long time. On the other hand, map development contains one intrinsic scale $t^{*}$ at which its governing exponent changes abruptly.

These findings offer an alternative interpretation for an observation central for our understanding of the orientation map, namely its apparent stability during development. The few chronic experiments reported in the past have been taken to suggest that the basic structure of orientation maps is preserved during development. In a pioneering experiment, correlation coefficients of $C \approx 0.5$ have been observed between post-natal day (PD) 35 and PD45 in the ferret [17]. Because of the presence of high spatial frequency noise, this value should be regarded as a lower bound of the true value. Coefficients around $C \approx 0.8$ between PD50 and PD120 were found in an analysis performed by the author. In the cat, coefficients up to $C \approx 0.8$ were observed between maps at PD35 and PD48 even for reversed- 
occluded animals [81] (however, without applying any high-pass filtering before calculating correlations). These experiments have raised the suspicion that orientation maps are prespecified by genetic instructions [21, 62, 79, 81]. Our analysis, however, shows that a developmental scenario based on self-organization is consistent with an apparently stable development of the orientation map.

In fact, our analysis shows that the model accounts even quantitatively for the observed similarities as suggested from the following considerations. In sensory systems so called critical periods have been observed, when the circuits underlying response properties (such as orientation selectivity or ocular dominance) are especially susceptible to changes of sensory input. Thus, the development of such properties is characterized by one intrinsic time scale. Stimulated by our results, we conjecture that the cross-over time $t^{*}$ is equal to this time scale for orientation selectivity. Unfortunately, this time scale is not known at present. Assuming that it is similar to the duration of the critical period of ocular dominance columns that terminates at approximately PD60 in the ferret (see Fig. 2.3) we can associate the stage $t=0$ with PD25 (the time orientation selectivity arises) and $t=t^{*}$ to PD60. For the classical ferret experiment mentioned above, the model predicts cross-correlations around $C \approx 0.8$ between maps recorded at these days (Fig. 6.2 which is even larger than the observed correlation of $C=0.5$. As outlined in this chapter, this prediction is largely independent of the model parameter $r$ and $g$.

Apart from demonstrating the consistency of the model with previous experimental results, our analysis suggests also ways to test its further predictions experimentally. In order to be able to observe any differences through measures of similarity, maps should be recorded at a very early phase in development. However, due to the growth of the visual cortex, chronic experiments during the first weeks are very difficult. Our analysis suggest that, if the experiment is performed at a very early stage, an acute experiments in which the map is recorded repeatedly within on session may be sufficient. An acute experiment usually provides better signal to noise ratios and introduces much less technical difficulties. To be able to observe the movement of pinwheels, our analysis suggest to record maps at exponentially increasing time intervals. 


\title{
7 Soft spots in the functional architecture of the visual cortex
}

\author{
The secrets of nature reveal \\ themselves more readily under \\ the vexation of art than when \\ they go their own way.
}

(Francis Bacon)

\subsection{Introduction}

Chapter 5 has shown that the pattern formation model (5.1) reproduces the pinwheel statistics reported in Chapter 4 . This supports the hypothesis that the correct model of the development of the orientation map is within the same universality class represented by this model. In this chapter, we explore further predictions of this model class and suggest ways to test them experimentally. In Chapter 8, we analyze data from experiments and compare them to the predictions of the model.

So far, stationary states and self-generated dynamics have been considered. However, properties of a dynamical system can often be well studied by perturbing it. Fortunately, since very recently, this is possible by electrical intracortical microstimulation (ICMS) which directly enforces local, intracortical synchronous activity. ICMS had been applied previously to various cortical areas such as the motor, somatosensory and auditory cortex, inducing plastic reorganization that remained localized and recovered completely shortly after stimulation. In their pioneering work, Godde et al. 35] worked out a paradigm for applying this stimulation protocol to adult cat visual cortex. They demonstrated an extensive restructuring of the layout of orientation maps up to several millimeters away from the ICMS site. Moreover, the induced changes showed a highly varying temporal dynamics ranging from complete recovery shortly after stimulation to progressive reorganization after ICMS and changes persistent up to 18hours. They suspected that both the high variability of the effect and the persistence of induced changes may be linked to the complex structure of the orientation map compared to the much more homogeneous organization of stimulus representations in e.g. the somatosensory cortex.

The framework of model (5.1) describes orientation maps as attractors of a nonlinear dynamics of cortical reorganization. This dynamics has multiple attractors characterized by different sets of active modes with different combinations 
of phases. Theoretically, it is conceivable that a perturbation may drive the orientation map into a new basin of attraction resulting in a global rearrangement of the pattern as it converges to the new attractor. Thus, within this framework the reorganization observed in ICMS experiments is interpreted as a switching between different attractor states. What stimulation would cause such switching? Due to the quasi-periodicity of the attractors, the reorganization induced by ICMS is expected to depend on the location of the ICMS site. If the orientation map had discrete translation symmetry (e.g. a hexagon or square pattern), this would also hold for the dependence of reorganization of the ICMS site. For quasi-periodic maps, however, the translation invariance is broken completely, such that no site of stimulation may be alike to any other. Moreover, this suggests that there are particular locations in the map that one might call soft spots, at which a localized perturbation induces strong and persistent reorganization. It is therefore an attractive hypothesis that the spatial dependency of ICMS efficiency is the primary origin of the high variability observed between experiments.

We begin this chapter by extending the generalized Swift-Hohenberg model (5.1) to mimic the effect of ICMS. In numerical simulations, we investigate the reorganization under ICMS including the dynamics of pinwheels. We identify soft spots and study their dependence on stimulation parameter and map layout for the full dynamics and, near criticality, by means of amplitude equations. We develop the quantity softness enabling a prediction of potential soft spots from the layout of the map.

\subsection{Modeling intracortical microstimulation (ICMS)}

The effect of ICMS is described in [35] (and references therein). Directly at the ICMS site the orientation preference remains constant. In its close vicinity, the preferred orientation is shifted towards the orientation at the ICMS site. Thus, locally ICMS has a enforcing effect on the orientation preference. The cortical region subject to the direct effect of ICMS is approximately $100 \mu \mathrm{m}$ which is an order of magnitude smaller than the spacing of orientation columns.

The effect of ICMS was examined in the phenomenological model (5.1) for the activity-driven dynamics of orientation maps introduced in Chapter 2 As outlined in Chapter 2, this model exhibits stable solutions which resemble orientation maps in the visual cortex. Since the aim is an understanding of the reorganization in the adult animal, the effect of ICMS on such stable solution is sought. To mimic the situation of an ICMS experiment, we modeled the application of ICMS by enforcing locally the preferred orientation. The full dynamics including the action of ICMS at cortical location $\mathbf{x}_{S}$ reads

$$
\partial_{t} z(\mathbf{x}, t)=L z(\mathbf{x}, t)+N[z(\mathbf{x}, t)]+\Theta(t) \Theta\left(t_{S}-t\right) S(\mathbf{x})
$$


where $\Theta$ is the Heaviside function, $t_{S}$ the duration of ICMS (starting at $t=0$ ). The first two terms on the r.h.s. denote the original model (5.1). A stable solution of the free dynamics will be denoted by $z_{\infty}(\mathbf{x})$ in the following.

In the model, ICMS is mimicked by an external force term. Consistent with the phenomenology of the experiment, ICMS shall enforce the preferred orientation at the ICMS site within a small cortical region. We thus modeled ICMS by choosing

$$
S(\mathbf{x})=\delta r z_{\infty}\left(\mathbf{x}_{S}\right) \mu\left(\mathbf{x}-\mathbf{x}_{S}\right)
$$

where $\delta$ is the ICMS strength and $\mu$ the stimulation function describing the spatial form of the direct ICMS effect. In the following, we use

$$
\mu(\mathbf{x})=\frac{1}{\pi \sigma_{S}^{2}} \exp \left(-\frac{\mathbf{x}^{2}}{2 \sigma_{S}^{2}}\right),
$$

a Gaussian with width $\sigma_{S}$. The direct effect of the force term Eq. (7.2) is to strengthen the selectivity $\left|z\left(\mathbf{x}_{S}\right)\right|$ around the stimulation site $\mathbf{x}_{S}$ and to widen the surrounding iso-orientation domain. Thus, for a given orientation map $z_{\infty}$ and ICMS site $\mathbf{x}_{S}$, ICMS is controlled by three parameters: The strength $\delta$ and duration $t_{S}$ of application, and the spatial width $\sigma_{S}$. The width is constraint by $\sigma_{S} \ll \wedge$ since the extension of the direct ICMS effect is expected to be small on the scale of the columnar pattern $\Lambda$. The duration $t_{S}$ shall be small on the scale $t^{*}$ on which a state $z_{\infty}$ close to the attractor is reached.

The implementation of ICMS in the form (7.2) can be interpreted as a simplification of a more accurate description. Including modulations of the map $z_{\infty}$ in the vicinity of the ICMS site and accounting for the temporal evolution of the stimulation the ICMS term may be written as

$$
S_{1}(\mathbf{x}, t)=\delta r z\left(\mathbf{x}-\mathbf{x}_{S}, t\right) \mu\left(\mathbf{x}-\mathbf{x}_{S}, t\right)
$$

with $z(\mathbf{x}, t=0)=z_{\infty}(\mathbf{x})$. Assuming that properties of the tissue (location of neurons, the surrounding glia cells, the concentration of ions, etc.) remain stable, the stimulation function can be taken as constant in time, $\mu(\mathbf{x}, t) \simeq \mu(\mathbf{x})$. If, in addition, the stimulation has a small range, the direction of stimulation can be approximated through $z(\mathbf{x}) \simeq z\left(\mathbf{x}_{S}\right)$ by the direction at the center. At pinwheel centers where the orientation preference is not continuous the selectivity approaches zero, such that the ICMS term becomes very small. Since the ICMS term enforces the selectivity around $\mathbf{x}_{S}$, the phase of $z\left(\mathbf{x}_{S}\right)$ will remain constant expecially if the complete system is translation and rotation invariant. Simplifying, one can use $z\left(\mathbf{x}_{S}, t\right) \simeq z_{\infty}\left(\mathbf{x}_{S}\right)$ thereby avoiding the positive feedback-loop in Eq. (7.4) for which there is no experimental evidence and which is undesirable from a technical perspective. Thus, apart from pinwheel centers where the modeling of ICMS is unclear for several reasons, both implementations Eq. (7.2) and Eq. (7.4) are 
expected to yield similar results. As will become apparent below, the basic mechanism underlying the effect of ICMS on the map is common for both forms.

\subsection{Methods}

\subsubsection{Numerical integration}

The semi-implicit numerical integration scheme used to integrate Eq. (7.1) without the ICMS term and its implementation are described in Section 7.2. The ICMS part 7.2) was treated explicitely. Writing

$$
\tilde{N}^{\prime}=\tilde{N}+\tilde{S}
$$

it was implemented in the Fourier domain as an additional term of the nonlinear part $\tilde{N}$ of Eq. 5.10. Formally, $\tilde{N}^{\prime}$ was treated as a correcting force term to the dynamics governed by the linear part $L$.

ICMS was applied to a near final state integrated until $t=10^{6}$ (in time scales of $t=1 / r$ ) using strengths ranging from $\delta=10^{-2.5}$ to $\delta=10^{-1}$ with durations between $t_{S}=10^{2}$ and $t_{S}=10^{3.5}$. After the termination of ICMS, the solution was advanced over $t=10^{4}$ to obtain a new stable solutions $z$. To calculate the induced modification $\Delta(\mathbf{x})$, the ICMS site was varied on a $50 \times 50$ grid in an area of $6.6 \wedge \times 6.6 \wedge$ within a larger map of size $17 \wedge \times 17 \Lambda$.

We used amplitude equations to study systematically the dependence on the ICMS parameter. Amplitude equations were integrated by an Adams-Gear scheme based on backward differentiation formula methods. The method is accurate in 5th order. The Jacobian matrix was approximated by divided differences. In parameter regimes with stable solutions of $n$ active modes the integration was performed for $2 n$ amplitudes by approximating the interaction coefficients by $g_{i i}=1, g_{i j}=$ $g, g_{i i^{-}}=2, f_{i j \neq i}=g / 2$ and $f_{i i^{-}}=1$. Before and after ICMS the solution was advanced to $t=10^{11}$. Further parameters were $g=0.98$, and $r=0.1$. Consistency test were carried out with arbitrary number of amplitudes (between 32 and 128) and the complete coefficient matrix.

\subsubsection{Pinwheel analysis}

Pinwheels were identified and tracked over time as described in Section 6.2.1. States were monitored at times $t_{i}$ separated by exponentially increasing time intervals $\Delta t_{i}=t_{i}-t_{i-1}$ between $t=0$ and $t=t_{S}$ during the period of ICMS and between $t=t_{S}$ and $t=t_{S}+10^{4}$ during relaxation after ICMS. A total of $N=100$ maps was sampled during each period. 

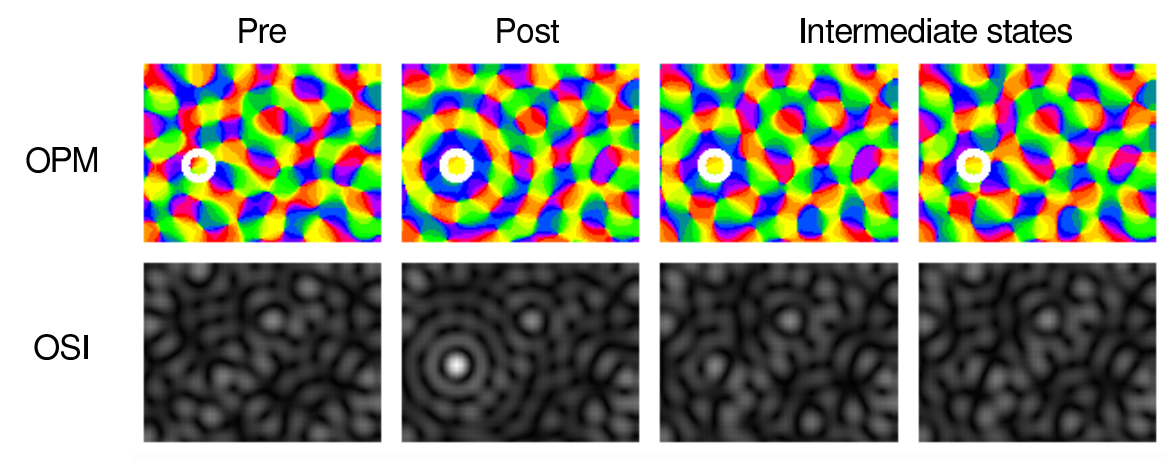

Final state
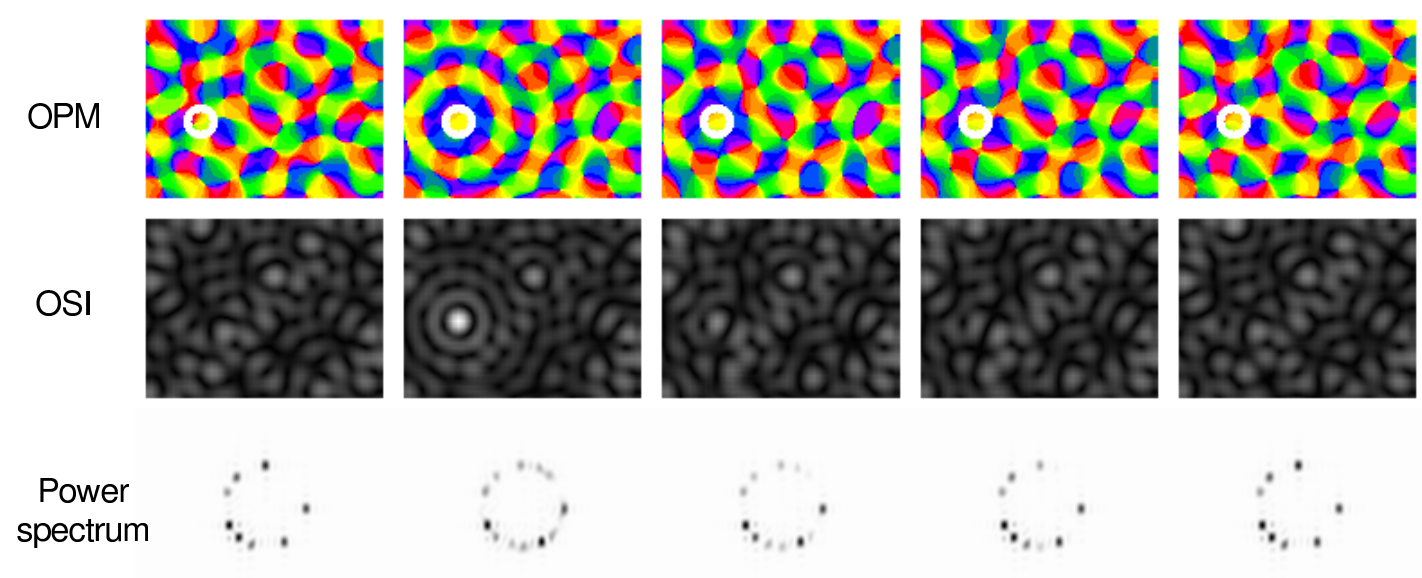

Local crosscorrelation
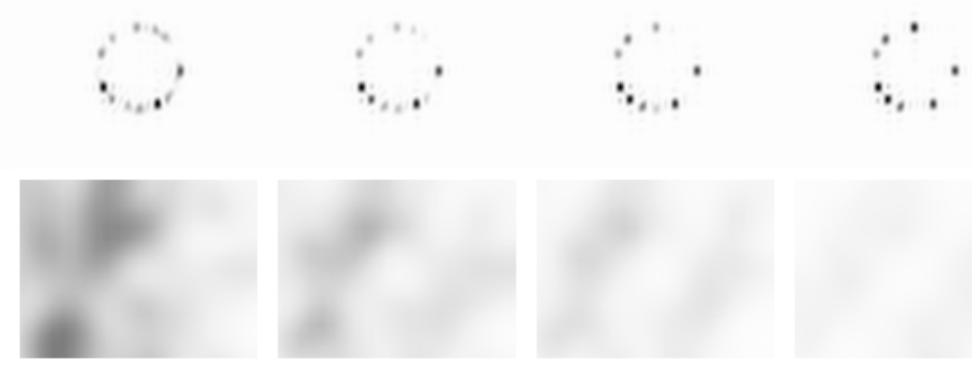

Figure 7.1: Transient changes induced by intracortical microstimulation (ICMS). Orientation preference map $\vartheta(\mathbf{x})(\mathrm{OPM})$, orientation selectivity $|z(\mathbf{x})|(\mathrm{OSI})$, and 2D power spectrum $|\tilde{z}(\mathbf{k})|^{2}$ (origin of k-space at the center of annulus) before (Pre) and at different stages after the application of ICMS (Post-Final state). Shown is a subregions of a larger map $(\Gamma=17)$ integrated on a $128 \times 128$ grid with $g=0.98, \sigma=1.7, \delta=10^{-2.9}, t_{S}=10^{2.7}$, advanced to $t=10^{6}$ before and $t=10^{4}$ after ICMS. For each state the local cross-correlation (Eq. 7.7.) with the initial map is shown in the lower row. Bright (dark) regions exhibit larger (smaller) correlations. Note that the initial and the final maps are very similar (average cross correlations: Pre/Post, $r=0.71$; Pre/Final, $r=0.95$ ) and exhibit identical power-spectra.

\subsection{A numerical ICMS experiment}

Starting point is the simulation of an ICMS experiment. An orientation map $z_{\infty}$ was prepared by integrating the free dynamics Eq. (5.1) up to $t=10^{6}$ using $r=0.1$, $g=0.98$ and $\sigma=1.7$. ICMS was applied as described in Section 7.3 (Eq. (7.1) with strength $\delta=10^{-2.9}$ and duration $t_{S}=10^{2.7}$. As in the real experiment, the orientation map was monitored immediately before and at various stages after ICMS. This Section shows for two representative examples the spatial-temporal reorganization induced by ICMS.

\subsubsection{Transient reorganization}

ICMS induced significant reorganization of the orientation map that continued after the end of application. Fig. 7.1 shows a numerical experiment in which ICMS 
has been applied to an attractor $z_{\infty}$ with $n=8$ active modes. Immediately after ICMS, the map appeared distorted with largest changes concentrated around the ICMS site. The domain surrounding the ICMS site was enlarged and numerous new modes on the critical circle $|\mathbf{k}|=k_{c}$ were activated as shown by the power spectrum $|\tilde{z}(\mathbf{k})|^{2}$ of the orientation map. At later stages the induced changes developed further while the map was relaxing towards a new stationary state. The power spectrum revealed that in this case, apart from a small phase shift, the original attractor recovered.

The induced spatial-temporal changes were quantified by means of local crosscorrelation between the attractor $z_{\infty}$ before ICMS and states developing after ICMS (Fig. 7.1). Maps $z(\mathbf{x}, t)$ were windowed at each location $\mathbf{x}^{\prime}$ by

$$
w\left(\mathbf{x}, \mathbf{x}^{\prime}, t\right)=g\left(\mathbf{x}-\mathbf{x}^{\prime}\right) z(\mathbf{x}, t)
$$

where $g$ is a Gaussian with $\sigma=0.6 \Lambda$. For every location $\mathbf{x}^{\prime}$, we calculated the cross-correlation between two windowed maps $w_{\infty}$ and $w_{2}$ according to

$$
C\left(\mathbf{x}^{\prime}\right)=\left\langle w_{\infty}\left(\mathbf{x}, \mathbf{x}^{\prime}, t\right) w_{2}\left(\mathbf{x}, \mathbf{x}^{\prime}, t^{\prime}\right)\right\rangle_{\mathbf{x}}
$$

yielding a map of local cross-correlation $C\left(\mathbf{x}^{\prime}\right)$ between the two maps. For the sake of numerical efficiency, maps were rescaled to the size of $1 / 6$ of the original maps. We found that the evoked changes were spatially inhomogeneous and maximal not in the immediate vicinity of the ICMS site. The average local cross-correlation between the initial and final map was $C=0.95$ confirming that the original map was largely recovered. In contrast, immediately after ICMS the correlation was reduced to $C=0.71$, indicating a transient reorganization.

\subsubsection{Persistent reorganization}

A very different effect was observed applying ICMS at an adjacent site. Fig. 7.2 shows a simulation carried out using the same attractor and identical stimulation parameters as for Fig. 7.1, but at a site shifted by $0.13 \wedge$. Compared to Fig. 7.1 . the effect immediately after ICMS was stronger and spatially widespread. Moreover, the map remained modified even after relaxation up to $t=10^{4}$ (right map in Fig. 7.2. A qualitatively new attractor developed as apparent from the power spectrum. Three of the modes active before the application of ICMS changed their location switching their wavevectors from $\mathbf{k}_{j}$ to $-\mathbf{k}_{j}$. This process results in a reduced cross-correlations of $C=0.67$ for the map immediately after ICMS and $C=0.70$ for the final map. The dissimilarity is spatially inhomogeneous reflecting the modulation due to the three deviating modes. Note that even though the change is substantial, it is not easily detected by inspection of the orientation preference maps. The power spectrum and the local cross-correlation provide a clearer descriptions of the effect which suggest to apply these analyses also to the experimental data (see Chapter 8). 

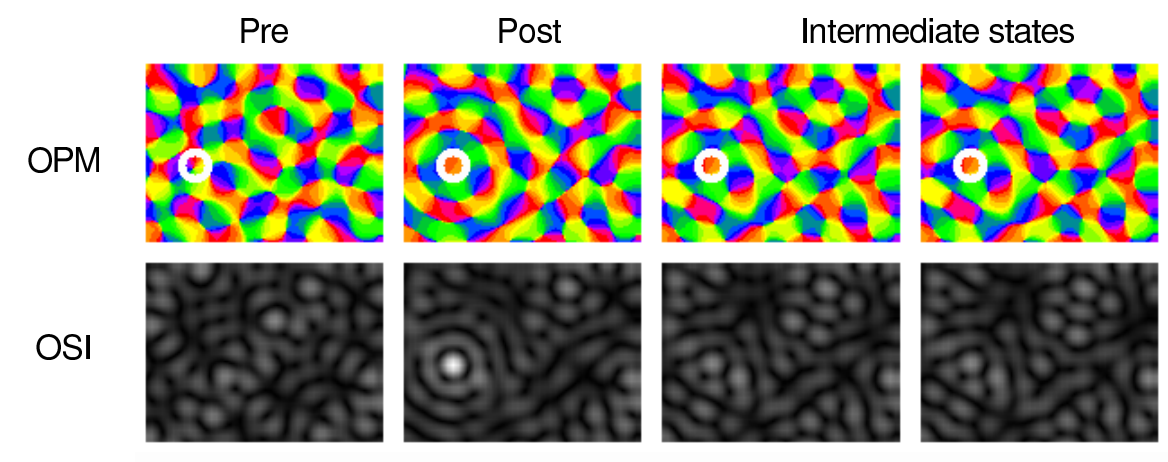

Final state
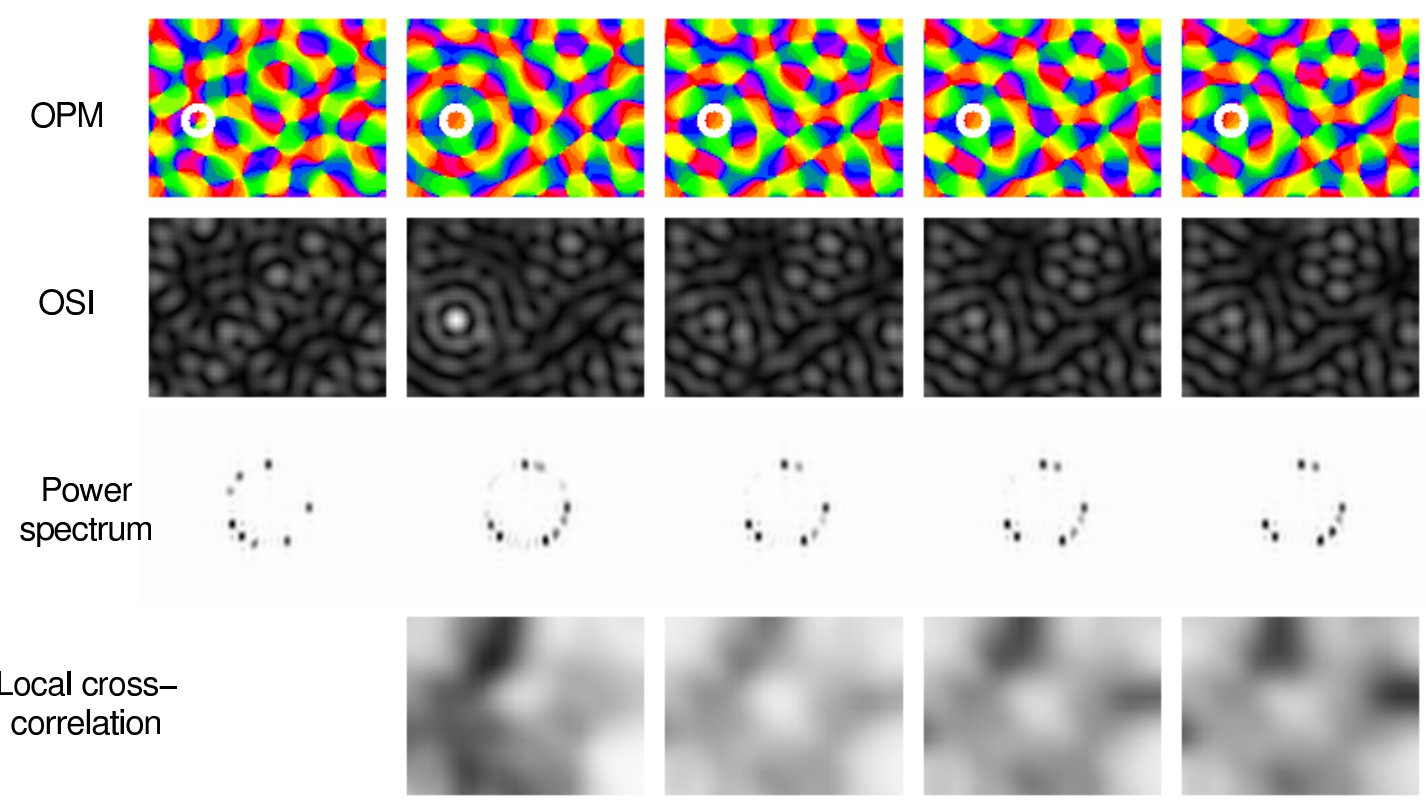

Figure 7.2: Persistent changes induced by ICMS. Application of ICMS as in Fig. 7.1 but to an adjacent site. Illustration as in 7.1. In contrast to 7.1 the final and initial map differ in many, but not all regions (Pre/Post, $r=0.67$; Pre/Final, $r=0.70$ ). Note from the power spectrum that modes have switched under the action of ICMS.

\subsubsection{Spatial-temporal dynamics}

The direct effect of ICMS is purely local. A spreading of the induced reorganization to remote sites is mediated by the local and non-local interactions in the model. The spreading is indicated by the maps of local cross-correlation in Fig. 7.1 and Fig. 7.2. To quantify the spreading of the ICMS effect, we averaged the local cross correlation $C(d \cos \theta, d \sin \theta)$ over angles,

$$
C_{0}(d)=\int_{0}^{2 \pi} \frac{d \theta}{d \pi^{2}} C(d, \theta),
$$

with the origin of space at the ICMS site. The ICMS distance dependent correlation $c_{0}(r)$ shows the spreading of changes (Fig. 7.3). In the two simulations from Fig. 7.1 and Fig. 7.2, changes were initially localized around the stimulation site. During prolonged application of ICMS, changes spread in a complex fashion to more remote regions. The largest difference was within a region of $\approx 3 \wedge$ reminiscent of the the distance covered by long-range connections of $\sigma=1.7 \wedge$. After ICMS, the evoked changes either started to shrink back and the initial map recovered or they developed further and a new map emerged. 

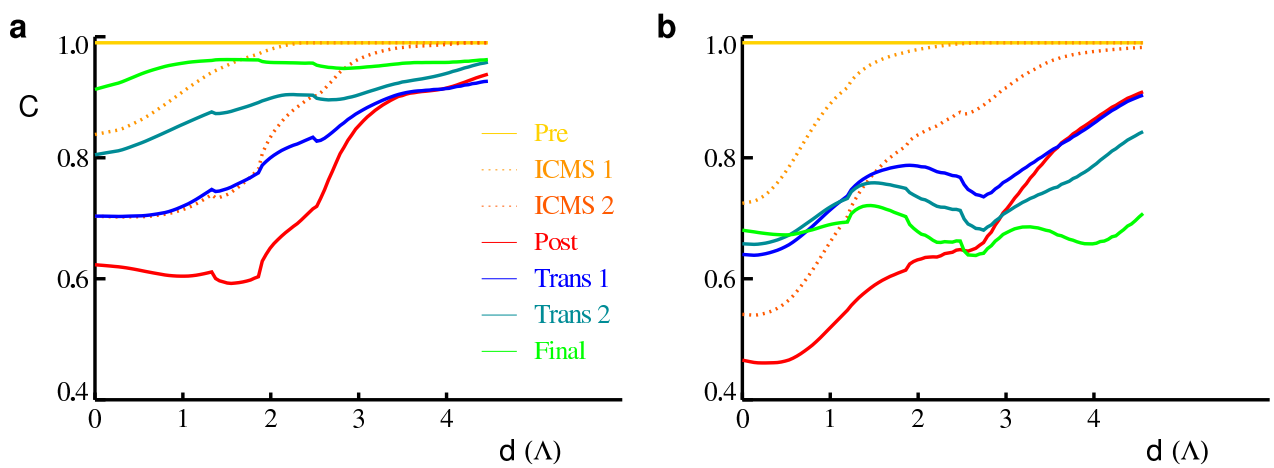

Figure 7.3: Spatial-temporal spreading of ICMS effect. a, b, Cross-correlations $C_{0}$ as a function of the distance $d$ ICMS site) for the data fro Fig. 7.1(a) and Fig. 7.2 (b). The initially localized reorganization spreads under persistent stimulation. Immediately after ICMS (Post), largest changes occur within a distance of $\approx 3 \wedge$ from the ICMS site. During the phase of relaxation after ICMS these regional changes either disappear largely (a) or a globally new state develops (b).

\subsection{Soft spots in the orientation map}

The two numerical ICMS experiments discussed in the previous Section 7.2 show that ICMS at nearby sites in the same orientation map can have very different effects on the orientation map. Whereas transient changes were induced in both cases, only for one case these changes were persistent. This suggests that a given orientation map contains soft spots, i.e. locations in the map where a stimulation such as ICMS is very effective in inducing large and persistent reorganization. In this Section, we identify the soft spots of a given map by systematically varying the location of the ICMS site in the map.

\subsubsection{Dependence of reorganization on ICMS site}

How does the strength of induced reorganization depend on the location of the ICMS site in the map? To explore this spatial dependency we systematically varied the ICMS site over a subregion of the map (see Method Section 7.3). Induced changes were quantified by

$$
\Delta\left(\mathbf{x}_{S}\right)=\left.\frac{1}{2|A|^{2}} \int_{\Omega} d^{2} \mathbf{k}|| \tilde{z}_{\infty}(\mathbf{k})\right|^{2}-\left|\tilde{z}_{\mathbf{x}_{S}}(\mathbf{k})\right|^{2} \mid
$$

called modification in the following. It is defined by the difference between power spectral densities of $z_{\infty}$ and $z_{\mathbf{x}_{S}}$ integrated over some area $\Omega$ containing the region of linear positive growth rate close the critical circle $\mathbf{k}=k_{c}$. With $A$ the amplitude 

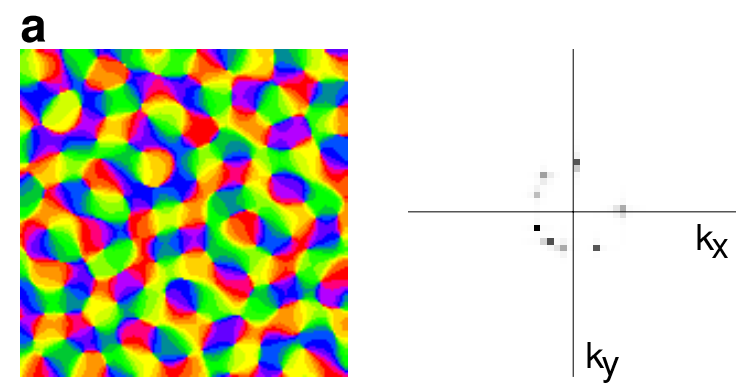

b

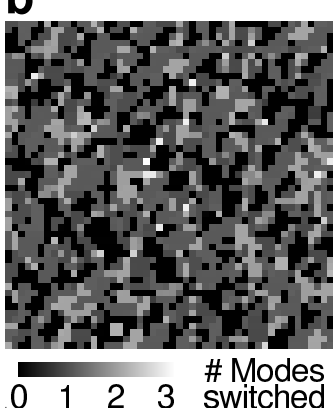

Figure 7.4: Soft spots where ICMS induces strong reorganization. a, Original orientation preference map $\vartheta_{\infty}(\mathbf{x})$ (left) and power spectrum $|\tilde{z}(\mathbf{k})|^{2}$ (right). b, Modification $\Delta\left(\mathbf{x}_{S}\right)$ (left; calculated as in Fig. 7.1 with $\delta=10^{-3}, t_{S}=10^{3}$ by systematically varying the ICMS site) and power spectrum $\left|\tilde{\Delta}\left(\mathbf{k}_{S}\right)\right|^{2}$ (right). Note that $\Delta$ strongly depends on the location of the ICMS site in the orientation map. Note further that the spatial organization of $\Delta$ bears no simple relation to the layout of the map. Its power spectrum contains most contributions on scales larger and smaller than the map.

of an active mode this measure counts the number of active modes switched from the action of ICMS. A nonzero value of $\Delta\left(\mathbf{x}_{S}\right)$ indicates the development of a new attractor with different modes active.

Fig. 7.4 shows the map of modification $\Delta\left(\mathbf{x}_{S}\right)$ together with the region of the map over which the modification was sampled. Interestingly, the modification $\Delta$ exhibited a complex spatial pattern bearing no resemblance to the layout of the underlying map. ICMS at nearby sites often had largely different effects. While inducing large layout changes at particular locations, applied to nearby sites the same stimulation could have no persistent effect whatsoever. Nevertheless, the modification $\Delta$ exhibited a large scale variation such that regions of the size of a few columns with enhanced modification alternate with regions of generally low modification. Consistently, the power spectral density of $\Delta$ (Fig. 7.4p) showed two prevalent spatial scales around $1 / 2 \Lambda$ and $2 \Lambda$. In contrast, the contribution on the scale $\Lambda$ of the orientation map appeared much weaker.

\subsubsection{Induced pinwheel dynamics}

Pinwheels were found to move and annihilate under the application of ICMS. During the induced reorganization of the map most pinwheel centers changed their location (Fig. 7.5a, b). We observed distances covered up to one mean column spacing. The range of movement only slightly depended on the distance to the ICMS site. The total number of pinwheels changed as a function of time during reorganization (Fig. 7.5k). A fraction of the pinwheels were annihilated. New pairs were created. Both the strength of these transient changes and the final result again 

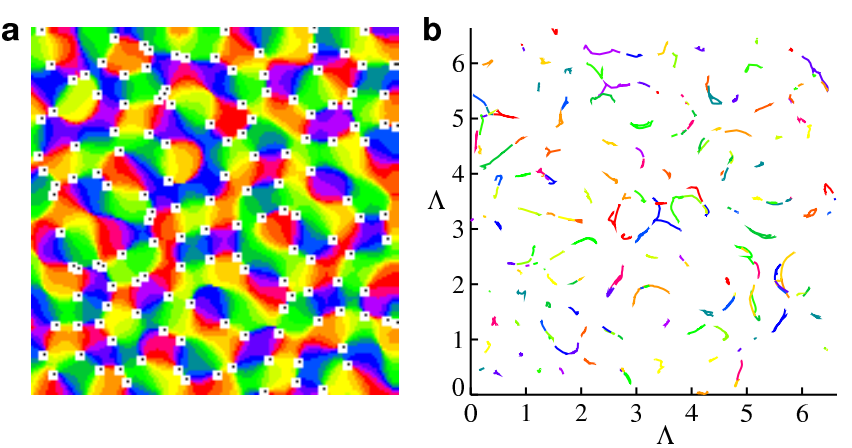

C
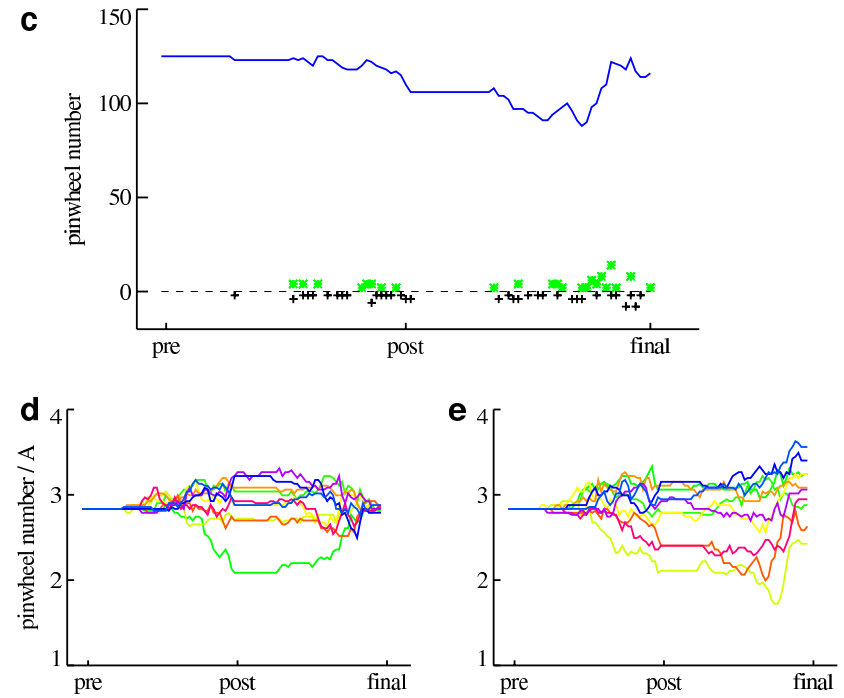

$\mathbf{f}$

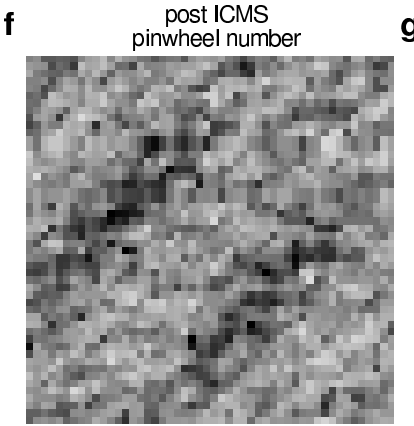

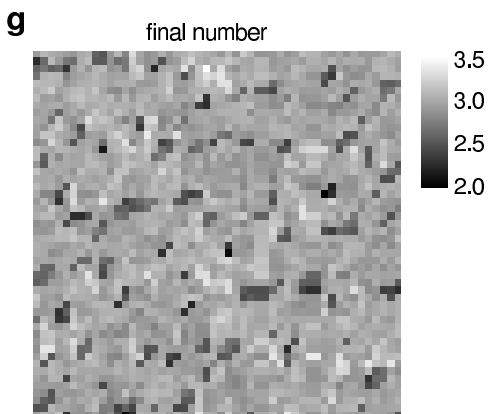

Figure 7.5: ICMS induced pinwheel movement, production and annihilation. a, Initial orientation preference map with marked pinwheel locations (subsection of the map simulated, smoothed for illustration). b, Movement of pinwheel centers during ICMS induced reorganization of the map in a (in mean column spacings 1). Different colors were used to discriminate pinwheels. c, Total number of pinwheels as a function of time during reorganization. Black crosses (green stars) mark the number of annihilation (creation) events. d, e, Pinwheel density (number per area $A=\Lambda^{2}$, with mean column spacing $\Lambda$ ) as a function of time during reorganization for a set of ICMS locations inducing transient reorganization of the map (d) and for ICMS locations inducing persistent changes of the map (e). $\mathbf{f}, \mathbf{g}$, Pinwheel numbers directly after termination of ICMS (f) and after convergence to a stationary state (g) as a function of the ICMS site.

strongly depended on the location of the ICMS site (Fig. 7.5 d-g). Final maps containing more pinwheels than initial maps were observed as well as cases with less pinwheels. Thus, ICMS induced complex dynamics of pinwheel locations sometimes leading to persistent changes of the qualitative structure of the map.

\subsubsection{Independence from local map properties}

One candidate for explaining the spatial scales occurring in the map of modification $\Delta$ is the orientation selectivity $\left|z_{\infty}\right|$. In fact, the strength of ICMS is propor- 
a

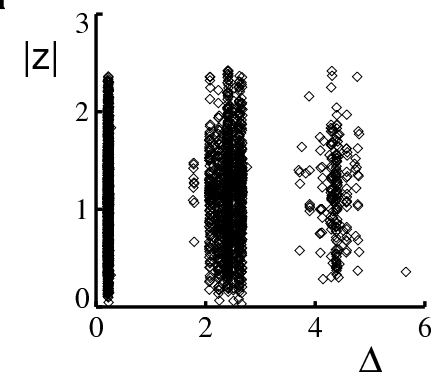

b

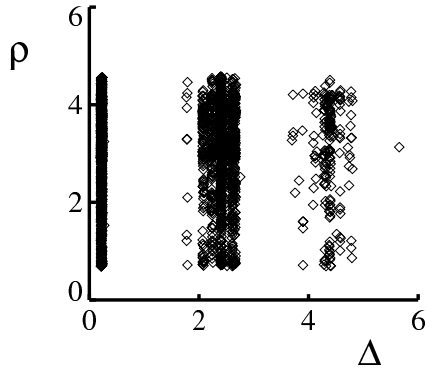

C

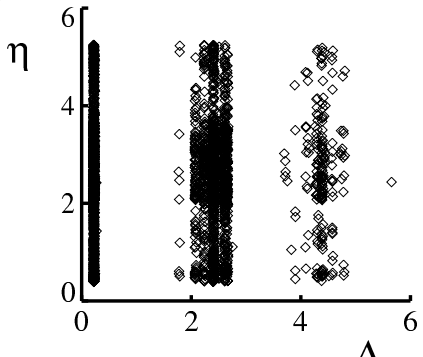

Figure 7.6: Relation of modification $\Delta$ to local map properties. a-c, The modification $\Delta$ is weakly anti-correlated to the orientation selectivity $\left|z_{\infty}\right|(C=-0.16)(\mathbf{a})$ and uncorrelated to the local density of pinwheels $\rho(C=-0.07)$ (b) and the local density of hyperbolic points $(C=-0.02)(\mathbf{c})$. Local densities in $\mathbf{b}, \mathbf{c}$ were calculated by smoothing densities with Gaussian kernel $(\sigma=\Lambda / 3)$.

tional to the selectivity $\left|z_{\infty}\right|$ (Eq. 7.2 ) and thus varies with ICMS location. Moreover, also the selectivity contains many spatial scales different from the scale of the map. Nevertheless, as shown in Fig. 7.6a, the correlation between the modification $\Delta$ and the orientation selectivity $\left|z_{\infty}\right|$ was only weak and negative $(C=-0.16)$. A large modification was mostly observed at locations with intermediate selectivity.

Other candidates are pinwheel centers and hyperbolic points which are both particular locations in the orientation map. Their spatial organization is less regular than the columnar layout and they exhibit broad nearest neighbor distributions (Section 5.4). Furthermore, at pinwheel centers the orientation preference is discontinuous. This suggests that the arrangement of pinwheels and hyperbolic points might be related to the modification $\Delta$. We tested this by analyzing the correlation between the modification $\Delta$ and the smoothed density of pinwheels $\rho$ and hyperbolic points $\eta$. As shown in Fig. 7.4b, c, no dependence was found on either of these quantities. This suggests that a more profound analysis is necessary in order to unravel the mechanism underlying soft spots in orientation map. Before addressing this, the dependency of the modification on stimulation parameters such as the ICMS duration $t_{S}$ and strength $\delta$ shall be studied in more detail.

\subsubsection{Stimulation strength and duration}

The location of soft spots within a map did not sensitively depend on the ICMS strength $\delta$ and its duration $t_{S}$. For $\delta$ or $t_{S}$ too small no changes were induced besides a shifting of phases $\phi_{j}$ of the active modes. Only if the strength and the duration were sufficiently large a switching of modes was observed at some sites. Increasing the strength or duration of application typically induced larger changes at these sites. However, an increase of either of these quantities did not always 

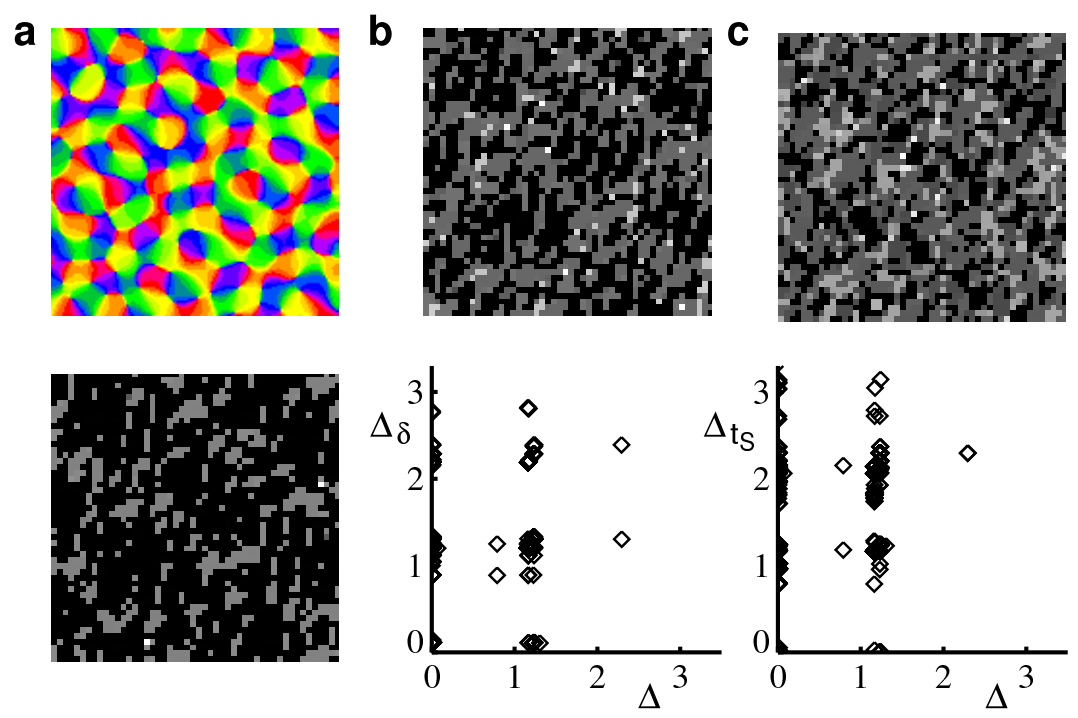

Figure 7.7: Dependence on ICMS strength $\delta$ and duration $t_{S}$. a, Map from Fig. 7.4 and modification $\Delta$ for $\delta=10^{-3}, t_{S}=10^{2.5}$, below. b, Increasing ICMS strength: Modification $\Delta_{\delta}$, for $\delta=10^{-2.5}, t_{S}=10^{2.5}$; below, scatter plot with $\Delta(C=0.48)$. c, Increasing ICMS duration: Modification $\Delta_{t_{S}}$, for $\delta=10^{-3}, t_{S}=10^{3}$; below, scatter plot with $\Delta(C=0.41)$. Note that dependent on the ICMS site $\mathbf{x}_{S}$, both an increase of ICMS strength or duration may result either in a larger or weaker modification, but its basic structure is relatively insensitive.

lead to a stronger effect. At particular ICMS sites $x_{S}$, the effect was always found to decrease with $\delta$ and $t_{S}$. Fig. $7.7 \mathrm{a}$ shows the modification $\Delta$ calculated for the orientation map from Fig. 7.4 using $\delta=10^{-3}$ and $t_{S}=10^{2.5}$. The modification $\Delta_{\delta}$ calculated for stronger ICMS of $\delta=10^{-2.5}$ exhibited a similar spatial structure, but with a larger average value (cross-correlation, $C=0.48$; Fig. 7.7p). Likewise, this was the case for the modification $\Delta_{t_{S}}$ calculated for an increased ICMS duration of $t_{S}=10^{3}(C=0.41$, Fig. 7.7 $)$. The depicted scatter plots of the two modifications $\Delta_{\delta}$ and $\Delta_{t_{S}}$ with $\Delta$ show that at particular sites, an increase of the ICMS strength or duration can also result in a weaker modification. However, the overall pattern of modification did not sensitively depend on the stimulation parameter. By means of amplitude equations, the parameter dependence will be studied in more detail in the following.

\subsubsection{Amplitude equations}

The numerical calculations presented in the previous sections are very time consuming. However, it is desirable to gain a better understanding of the dependence of the ICMS effect on stimulation parameter such as ICMS strength $\delta$ and duration $t_{s}$. To achieve this, we formulate ICMS in terms of the amplitude formalism. 
Within this approximation, which remains accurate for small $r$, the dynamics become much more tractable numerically. Moreover, the reduced dynamics of amplitudes will provide an improved conceptual understanding of the mechanisms underlying the observed reorganization induced by ICMS as shall be outlined in the Section 7.7 .

In Fourier representation the ICMS term Eq. (7.2) reads

$$
\tilde{S}(\mathbf{k})=\delta r z_{\infty}\left(\mathbf{x}_{S}\right) \tilde{\mu}(\mathbf{k})
$$

where $\tilde{\mu}$ is Fourier transform of the stimulation function (7.3). For the upcoming analysis it is convenient to shift the ICMS site $\mathbf{x}_{S}$ to the origin of the coordinate system. The stimulation function then simply becomes the Gaussian

$$
\tilde{\mu}(\mathbf{k})=\exp \left(-\frac{\sigma_{S}^{2} \mathbf{k}^{2}}{2}\right)
$$

with width $1 / \sigma_{S}$. Since this width is large compared to the typical wavenumber $k_{c}=2 \pi / \Lambda$ we may set $\tilde{\mu}\left(k_{c}\right)=1$ for simplicity. With Eq. (7.11) the ICMS term (7.10) exhibits a uniform complex phase

$$
\phi_{\infty}\left(\mathbf{x}_{S}\right)=\arg \left\{z_{\infty}\left(\mathbf{x}_{S}\right)\right\}
$$

which is determined by the orientation preference at the ICMS site by $\phi_{\infty}\left(\mathbf{x}_{S}\right)=$ $2 \vartheta\left(\mathbf{x}_{S}\right)$.

Hence, the amplitude equations accurate at order $r^{3 / 2}$ read

$$
\partial_{t} A_{i}=r A_{i}-\sum_{j} g_{i j}\left|A_{j}\right|^{2} A_{i}-\sum_{j} f_{i j} A_{j} A_{j^{-}} \bar{A}_{i^{-}}+\Theta(t) \Theta\left(t_{S}-t\right) \delta r z_{\infty}\left(\mathbf{x}_{S}\right)
$$

governing the development of amplitudes $A_{j}=\left|A_{j}\right| e^{i \phi_{j}}$ under the presence of ICMS between $t=0$ and $t=t_{S}$. Eq. (7.13) is for $0 \leq t \leq t_{S}$ a set of inhomogeneous equations. The ICMS term in (7.13) acts as an additional force with constant strength and constant phase on the modes on the active amplitudes $A_{i}$. For very small strength $\delta$ this force will mainly shift the phases $\phi_{j}$ of the active amplitudes towards the ICMS phase $\phi_{\infty}\left(\mathbf{x}_{S}\right)$. For sufficiently large $\delta$ additional amplitudes on the critical circle may grow or previous active amplitudes are suppressed. In the following, the dependency of the ICMS effect on the two stimulation parameters strength $\delta$ and duration $t_{S}$ shall be evaluated using amplitude Eq. (7.13).

\subsubsection{Modification diagram}

The modification diagram displayed in Fig. 7.8 $\mathrm{B}$ captures the dependence of the mean modification $\Delta=\langle\Delta(\mathbf{x})\rangle_{\mathbf{x}}$ induced by ICMS on its two parameters ICMS 

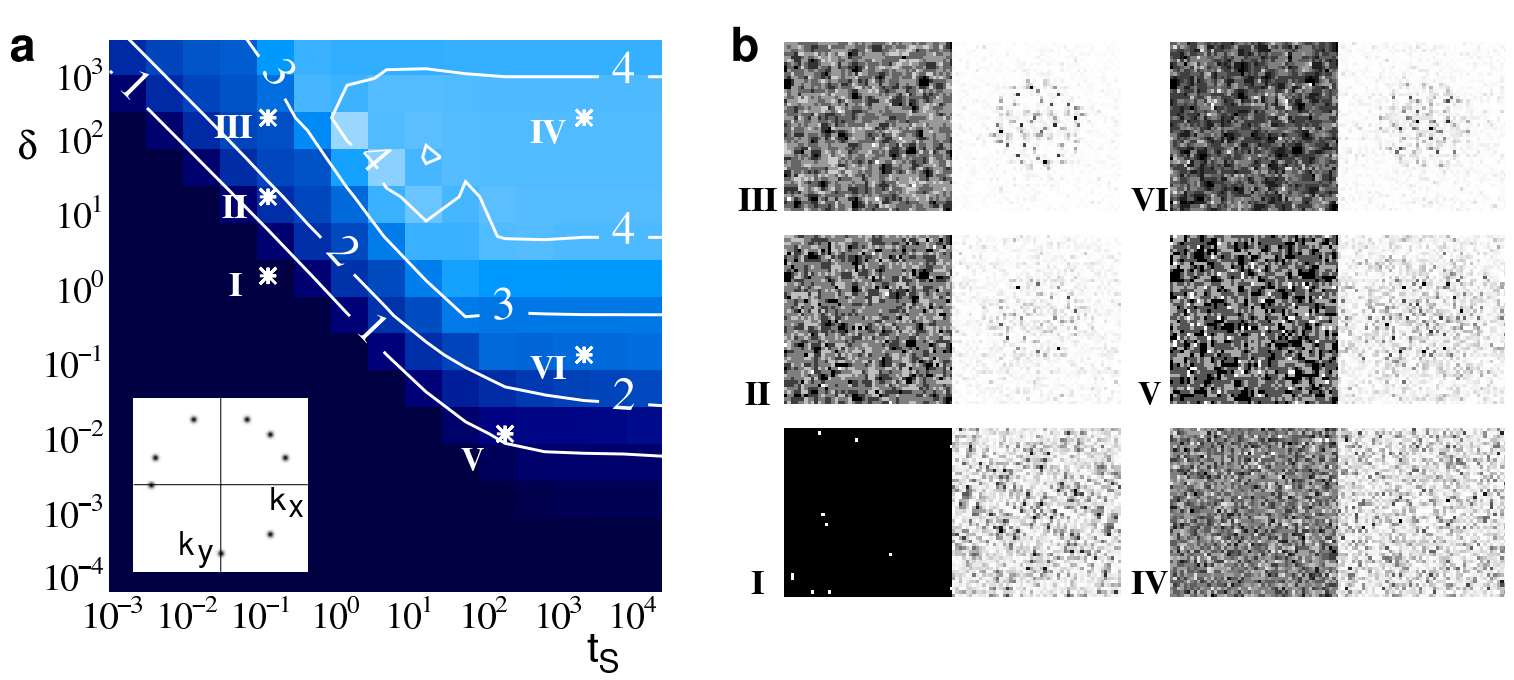

Figure 7.8: Modification diagram: Dependence of modification $\Delta$ on ICMS duration $t_{S}$ and strength $\delta$. a, Mean modification $\Delta=\left\langle\Delta\left(\mathbf{x}_{S}\right)\right\rangle_{\mathbf{x}_{S}}$ calculated by the amplitude equations 7.13 ICMS was applied to a planform solution with $n=8$ active modes (inset). b. Modification $\Delta$ and its power spectrum $|\tilde{\Delta}|^{2}$ (illustration as in Fig. $7.4 \mathrm{p}$ ) for different parameter regimes (marked by stars in a). In the small $\delta, t_{S}$ regime the configuration of active modes remains unchanged under ICMS. For sufficiently large $\delta$, a single mode is flipped for some ICMS sites $\mathbf{x}_{S}(I)$. Under very strong stimulation, 4 modes switch on average $(I V)$. Soft spots are found in the parameter regime of moderate modification given by $10^{-1} \lesssim \delta t_{S} \lesssim 10^{2}$ for $t_{S}<10^{2}$ and $10^{-3} \lesssim \delta \lesssim 10^{0}$ for $t_{S}>10^{2}$. Note that $\Delta$ is similar for $I I$ and $V(c=0.66)$ and for $I I I$ and $V I(c=0.55)$.

strength $\delta$ and duration $t_{S}$. Calculations were carried out using a planform of $n=8$ active modes. For short and weak stimuli the modification was close to zero - the stimulation failed to switch any of the active modes. For large durations $t_{S}>$ $10^{3}$, the modification depended on $\delta$ only. In fact, there was a minimal strength $\delta_{\min } \approx 10^{-3}$ necessary for inducing any modification at all in a map. A nonzero modification was obtained for strengths as small as $\delta>10^{-3}$ for duration $t_{S}>10^{2}$ or for $t_{S}<10^{2}$ for strengths fulfilling $\delta t_{S}>10^{-1}$. For larger $\delta$, the mean effect $\Delta$ was found to increase gradually over more than three orders of magnitude of ICMS strengths $\delta$. The maximal effect was a switching of more than half of the originally active modes. In this regime ICMS induced an activation of all $n=$ 16 amplitudes to an almost equal amplitude. The selection of the finally active modes after termination of ICMS becomes essentially a random process in which on average $n=4$ modes switch.

Thus, soft spots are only found in the parameter regime in which usually a fraction of the possible modes are switched. This regime of moderate modification, is therefore of particular interest in the following. As will become apparent later in this chapter, in this regime, the modification can be predicted to some degree from the ICMS site in the map. The strength of the modification $\Delta$ increases gradually 

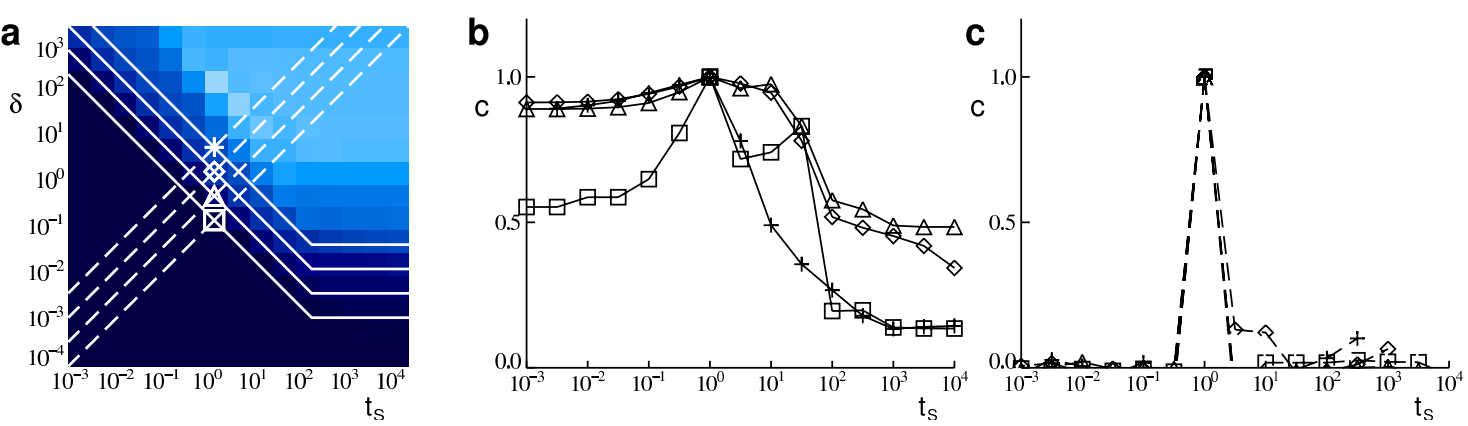

Figure 7.9: The modification $\Delta$ largely depends on the product $\delta t_{S}$ of ICMS strength and duration. a, Traces of cross-correlations $C$ parallel (solid) and perpendicular (dashed) to the level lines of equal mean modification $\Delta$. The four reference modifications at $t_{S}=10^{0}$ and $\delta=10^{-1}, 10^{-1 / 2}, 10^{0}, 10^{1 / 2}$ are marked by different symbols. b. Correlations parallel to level lines vs. ICMS duration $t_{S}$ remain large over a broad width. c, Correlations perpendicular to level lines decay rapidly.

with the strength $\delta$ and duration $t_{S}$ of ICMS in this regime. Fig. 7.8p shows the representative maps of modification $\Delta$ from 6 different regions of the modification diagram. Whereas the spatial dependence of stimulation is essentially random for very large and strong stimulation, in the regime of moderate modification, it exhibits an intriguing spatial dependence resembling the results form the numerical calculations of the full dynamics (compare Fig. 7.4 and Fig. 7.7). As for the full dynamics, in this regime the modification $\Delta$ varies on spatial scales smaller and larger than the pattern itself as will be discussed in further detail in Section 7.7.1.

Fig. 7.8 shows a further property of the modification $\Delta$ that is worth noting. The spatial structure changes only little when moving along the level lines in the modification diagram. The modification maps II and $V$ were significantly correlated $(c=0.66)$ despite their relatively large distance in parameter space. A similar correlation was found for III and VI $(c=0.66)$. To evaluate this more systematically we calculated cross-correlations of the maps of modification parallel and perpendicular to the equal effect level lines starting from four maps within the sensitive regime at equal time $t=10^{\circ}$, but with various strength $\delta$ (Fig. 7.9). Along the lines of equal mean modification their spatial structure was largely reproducible, especially along the line given by $\delta t_{S}=1$ with correlations close to 1 (Fig. 7.9p, solid lines in a). The spatial structure of the modification $\Delta$ was much less similar along the perpendicular direction and correlations decayed rapidly (Fig. 7.9k, dashed lines in a). Thus, the combination of stimulation strength and duration mainly determines the induced modification. For a duration $t_{S}<10^{3}$ the result depends to a good approximation only on the product $\delta t_{S}$. 
a
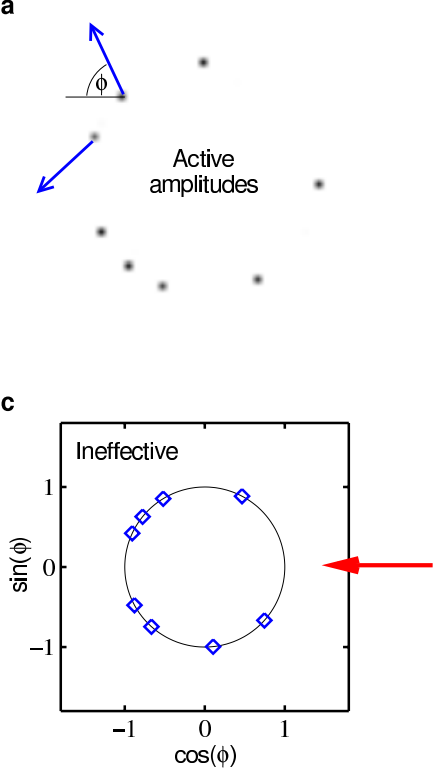

b
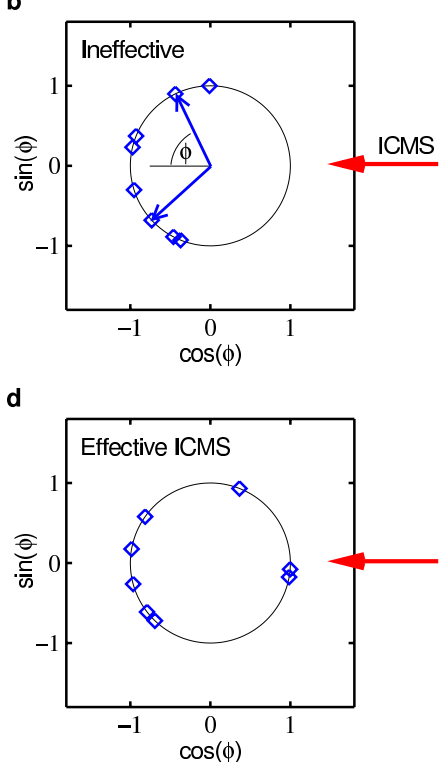

Figure 7.10: Layout of orientation map implies the occurrence of soft spots. a, Fourier representation of map before ICMS (schematic). Active amplitudes with moduli $\left|A_{j}\right|$ (black dots) and phases $\phi_{j}$ (blue arrows, shown for two amplitudes). b, c, Phase condition for ICMS sites with small modification $\Delta$. Under ICMS, phases $\phi_{j}$ (diamonds) are shifted towards the ICMS phase $\phi_{\infty}=\pi$ (red arrow) strengthening all active amplitudes. d, Phase condition at ICMS site with large $\Delta$. Here, two amplitudes are suppressed and can therefore be exchanged by different amplitudes.

\subsection{Soft spots implied by map layout}

Section 7.5 has shown that the reorganization induced by ICMS strongly depends on the location of the stimulation-site in the map. Other parameters being equal, ICMS at adjacent locations often results in a qualitatively different outcome as highlighted in Fig. 7.4. Moreover, this spatial dependency did not depend sensitively on the parameter of the stimulation. This suggest that, despite any apparent resemblance to the orientation map $z_{\infty}$, the modification $\Delta$ depends to a considerable degree on the arrangement of columns.

Consider an orientation map in the form

$$
z_{\infty}(\mathbf{x})=\sum_{j=0}^{n-1} \mathcal{A}_{j} e^{i \mathbf{k}_{j} \mathbf{x}+i \phi_{j}}
$$

composed of $n$ active modes $j$ with amplitudes $A_{j}=\mathcal{A} e^{i \phi_{j}}$ (Fig. 7.10a). Let us assume that all amplitudes have an equal absolute value $\mathcal{A}_{j}=\mathcal{A}$ (which is accurate within the approximation of amplitude equations) and that the ICMS site is at the origin, $\mathbf{x}_{S}=\mathbf{0}$. According to Eq. (7.10), ICMS acts with uniform phase $\phi_{\infty}$ on active and inactive amplitudes. Following

$$
\left|z_{\infty}\right| e^{i \phi_{\infty}}=\mathcal{A} \sum_{j=0}^{n-1} e^{i \phi_{j}}
$$


this phase results from the complex superposition of active amplitudes. Consequently, the majority of phases $\phi_{j}$ points towards the direction of the the ICMS phase $\phi_{\infty}$ (as in Fig. 7.10p, c). Under the influence of ICMS, these amplitudes shift their phase towards $\phi_{\infty}$ and grow in modulus. Thus, they get further enhanced thereby suppressing through nonlinear competition the growth of new, previously inactive amplitudes finally preventing a switching to a new attractor. As illustrated in Fig. 7.10 d, ICMS successfully suppresses an active amplitude $A_{j}$ if the phase condition

$$
e^{i \phi_{j}\left(\mathbf{x}_{S}\right)} \propto-e^{i \phi_{\infty}\left(\mathbf{x}_{S}\right)}
$$

is fulfilled, i.e. if the phase of the amplitude is opposed to the phase of ICMS. Given that the phase shift of $\phi_{j}$ is very slow, the amplitude is suppressed by the now opponent ICMS force. If sufficiently suppressed, a new growing mode may eventually exceed this amplitude by the end of the ICMS application. Depending on the strength and duration of ICMS and the details of the governing dynamics of the model this can lead to the observed switching. The state enters a different basin of attraction and finally relaxes towards a different stable solution.

How does the dependence on the location of the ICMS site arise? The phases of the active amplitudes change as

$$
\phi_{j} \rightarrow \mathbf{x}_{S} \cdot \mathbf{k}_{j}+\phi_{j}
$$

with ICMS location $\mathbf{x}_{S}$. Thus, dependent on the projection of $\mathbf{k}_{j}$ on $\mathbf{x}_{S}$, phases $\phi_{j}$ change on scales equal or larger than $\Lambda$ which may explain the occurrence of long scale contributions to the modification $\Delta$ (see e.g. Fig 7.4). The occurrence of small scales in $\Delta$ at approximately $\Lambda / 2$ (Fig 7.4) may be understood from reconsidering Fig. $7.10 \mathrm{~d}$. A similar phase constellation at a different site is possible only if it is $\geq \Lambda / 2$ remote, because a phase shift of $\pi$ is necessary in order to obtain two amplitudes with phases opposite to $\phi_{\infty}$. The importance of the phase constellation also suggests why spots of large modification $\Delta$ are rare in a map. By the enforcing nature of ICMS, phases $\phi_{j}$ tend to point towards the ICMS phase. For one amplitude condition (7.16) only holds at a subsample of ICMS sites and the probability to hold for more than one amplitude is much smaller. Moreover, a fraction of amplitudes satisfying the phase condition (7.16) requires an even larger fraction concentrated around $\phi_{\infty}$. In fact, the maximum number of phases fulfilling Eq. (7.16) can never exceed half of the total number of active modes. Thus, for inducing a large reorganization in the orientation map, the ICMS site must be chosen at a very particular location.

The mechanism described in this Section shows that the property of soft spots arises naturally from the spatial structure of the orientation map. It is a consequence of its various component modes. At least for moderate stimulation the phase relation (7.16) provides a necessary condition for inducing significant reorganization: Where it is not fulfilled, a persistent ICMS effect is not expected; where it 
is satisfied, the map undergoes persistent reorganization or not, depending on the details of the dynamics governing its evolution. Thus, the modifiability through ICMS is constraint by the layout of the map.

\subsection{Predicting soft spots}

Section 7.5 showed that the induced reorganization strongly depended on the ICMS site in the map, but less on ICMS parameter stimulation strength and duration. It was suggested to depend to some extent on the layout of the orientation map leading to the notion of soft spots. In the previous Section 7.6. we proposed a mechanism by which the layout of the map can influence the efficiency of ICMS at a given site in evoking persistent reorganization. In this Section, we develop methods for analyzing the validity of this mechanism. It is suggested that the effect of ICMS can be predicted to some degree from the layout of the map.

\subsubsection{Softness}

To evaluate the implication of the simple phase condition $(7.16)$ for the convertibility of a map by ICMS, it is convenient to express it by a real scalar function which is called softness $\kappa$ in the following. The softness $\kappa$ shall become larger the more amplitudes fulfill the phase condition and small at sites where it does not hold for any of the active amplitudes. Furthermore, it shall be a function of the modes $\phi_{j}$ only. We defined the softness by

$$
\kappa\left(\mathbf{x}_{S}\right)=S D\left(\cos \left[\phi_{j}\left(\mathbf{x}_{S}\right)-\phi_{\infty}\left(\mathbf{x}_{S}\right)\right]\right)
$$

where $S D()$ denotes standard deviation. The cosine in the brackets is -1 for amplitudes $A_{j}$ for which the phase condition Eq. (7.16) is fulfilled, and +1 if the phase is identical to the ICMS phase. The softness $\kappa$ is large if the phases $\phi_{j}$ exhibit a bimodal distribution on the unit circle (as in Fig. 7.10d). It is small, if phases $\phi_{j}$ are rather uniformly distributed (Fig. $7.10 \mathrm{k}$ ) and very small for a unimodal distribution (Fig. $7.10 \mathrm{p}$ ). The quantity $\kappa$ increases generally with the number of modes fulfilling the phase condition.

Fig. 7.11 shows a comparison of the softness $\kappa$ to the modification $\Delta$ calculated by the amplitude equations as in Fig. 7.8 ( with $t_{S}=10^{2.5}, \delta=10^{-2}$ ). The modification (Fig. 7.11) highly resembled the map of softness $k$ (Fig. 7.11b). Especially for regions of low modification and softness (dark regions in Fig. 7.11a, b), the similarity was strong consistent with the interpretation of the phase condition (7.16) as a necessary condition for inducing large reorganization. The relation of $\Delta$ and $\kappa$ is depicted in Fig. 7.11k (cross-correlation, $C=0.71$ ). Thus, the softness $\kappa$ can indeed successfully predict the modification $\Delta$ at least when obtained from the amplitude equations. 

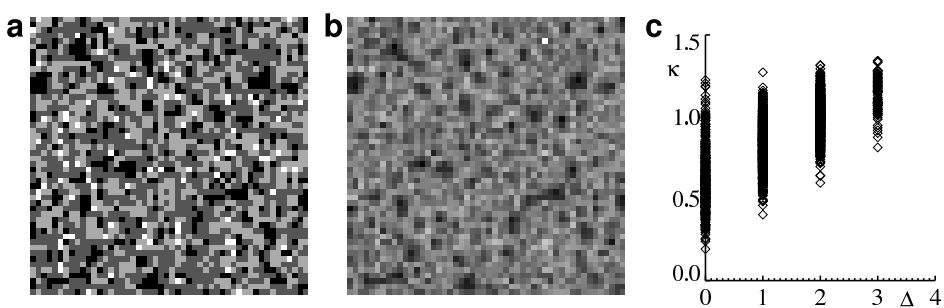

d

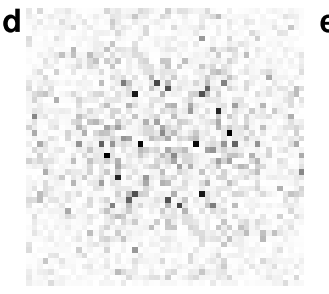

e f

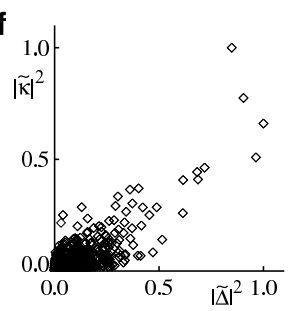

Figure 7.11: The predictive power of the softness $\kappa$. $\mathbf{a}, \mathbf{b}$, Modification $\Delta$ for the map from Fig. $7.8\left(t_{S}=10^{2.5}, \delta=10^{-2}\right)$ (a) and softness $\kappa($ b). Dark (bright) regions indicate small (large) values of $\Delta$ and $\kappa$, respectively. c, The spatial structure of $\kappa$ predicts $\Delta$ to a high degree (cross-correlation, $C=$ 0.71). Their power spectra $|\tilde{\Delta}|^{2}$ (d) and $|\tilde{\kappa}|^{2}(\mathbf{e})$ share most prevalent scales $(C=0.77)(\mathbf{f})$.

Before further pursuing this approach, it is interesting to consider the spatial scales on which the softness $\kappa$ varies. Using Eqs. (7.15) and (7.17) we rewrite (7.18) into

$$
\begin{aligned}
\kappa\left(\mathbf{x}_{S}\right) & =S D\left(\frac{\mathcal{A}}{\left|z_{\infty}\left(\mathbf{x}_{S}\right)\right|} \sum_{l} \cos \left[\phi_{l}\left(\mathbf{x}_{S}\right)-\phi_{j}\left(\mathbf{x}_{S}\right)\right]\right) \\
& =S D\left(\frac{\sum_{l} \Phi_{l j}}{\sqrt{\sum_{l i} \Phi_{l i}}}\right)_{j}
\end{aligned}
$$

where

$$
\Phi_{l j}=\cos \left[\left(\mathbf{k}_{l}-\mathbf{k}_{j}\right) \mathbf{x}_{S}+\phi_{l}^{0}-\phi_{j}^{0}\right]
$$

with $\phi_{j}^{0}=\phi_{j}\left(\mathbf{x}_{S}=\mathbf{0}\right)$. Following Eq. 7.19$)$, the softness $\kappa\left(\mathbf{x}_{S}\right)$ comprises a variety of spatial scales generated through multiple combinations of wavevectors $\mathbf{k}_{j}-\mathbf{k}_{i}$. Depending on the attractor, already these constituent wavevectors vary in their modulus within $0 \leq\left|\mathbf{k}_{j}-\mathbf{k}_{i}\right| \leq 2 k_{c} \cos (\pi / 2 n)$, i.e. on scales between $\Lambda / 2$ and $\infty$ in the large $n$ limit, but even larger frequencies are generated from these wavevectors by Eq. (7.19). As demonstrated in Fig. 7.11f the range of scales in the powerspectrum of the modification, $|\tilde{\Delta}(\mathbf{k})|^{2}$ (Fig. $7.11 \mathrm{~d}$ ), is shared by the spectrum of the softness, $|\tilde{K}(\mathbf{k})|^{2}$ (Fig. 7.11 ). Thus, the phase condition 7.16 in the form of the softness $K$ can indeed account for the observed spatial dependency of the induced modification $\Delta$.

\subsubsection{Predictability}

The softness $k$ provides a means of predicting the effect of stimulation to some extent without knowing the dynamics of the system. The extent to which this is 

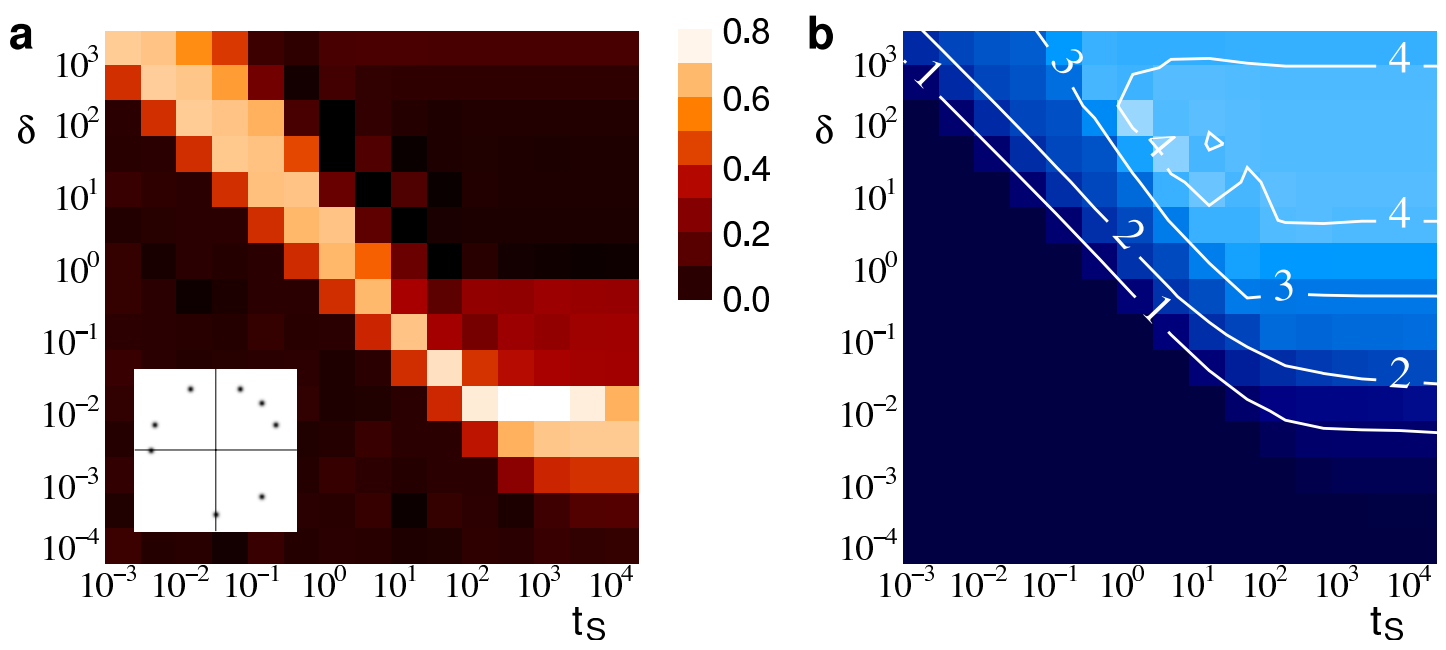

Figure 7.12: Parameter regime of successful prediction of reorganization. a, Diagram of predictability $\mathcal{P}$ of modification from softness $\kappa$ : Dependence on ICMS duration $t_{S}$ and strength $\delta$ (in red scales). b, Effect diagram from Fig. 7.8. The strength of induced reorganization can be predicted in the parameter regime of moderate reorganization.

possible, denoted by the predictability $\mathcal{P}$, is quantified as the cross-correlation between modification $\Delta\left(\mathbf{x}_{S}\right)$ and softness $\kappa\left(\mathbf{x}_{S}\right)$. A value of $\mathcal{P}$ close to 1 indicates a high similarity between both quantities. A value close to 0 indicates no predictive power of the softness $\kappa$. The predictability $\mathcal{P}$ may depend on the duration $t_{S}$ and strength $\delta$ of ICMS. Comprehensively, this can be studied only by the amplitude equations which are much more tractable both analytically and numerically. Therefore, we first focus on this approximation before investigating the full dynamics.

Fig. 7.12a shows the diagram of predictability $\mathcal{P}$ for the attractor from Fig. 7.8 together with the diagram of mean modification for the same range of ICMS strength $\delta$ and duration $t_{S}$ as in Fig. 7.8. A . Across the parameter regime of moderate modification we observed a high predictability $\mathcal{P}$. Values of $\mathcal{P}>0.6$ were found in the regime $\delta t_{S} \approx 1$, especially for small strengths of $\delta \approx 10^{-2}$ and large durations of $t_{S} \gtrsim 10^{2}$ with predictabilities up to $\mathcal{P}=0.75$. The predictability was low only in the regime of no persistent modification and in the regime of maximal modification where all amplitudes are equally activated by a strong external stimulation.

What gives rise to this large predictability in the regime $\delta t_{S} \approx 1$ ? An improved understanding of the effect of ICMS can be obtained by linear response analysis. Starting point are the amplitude equations in the form Eq. (7.13). Linearizing around a planform solution

$$
\begin{aligned}
A_{i} & =\mathcal{A} e^{i \phi_{i}^{0}}, i=0, \ldots, n-1 \\
B_{i} & =0, \quad i=i, \ldots, n-1
\end{aligned}
$$

and splitting the ICMS term into the part acting on the amplitudes and that acting 
on the phases leads to equations

$$
\begin{aligned}
& \dot{a}_{i}=-2 \mathcal{A}^{2} \sum_{j} g_{i j} a_{j}+\delta r\left|z_{\infty}\right| \cos \left(\phi_{\infty}-\phi_{i}^{0}-\theta_{i}\right) \\
& \dot{\theta}_{i}=\delta r\left|z_{\infty}\right| \sin \left(\phi_{\infty}-\phi_{i}^{0}-\theta_{i}\right)
\end{aligned}
$$

for the small amplitudes $a_{i}(t)=\mathcal{A}_{i}(t)-\mathcal{A}$ and phases $\theta_{i}(t)=\phi_{i}(t)-\phi_{i}^{0}$ where in the first equation we used $r=\mathcal{A}^{2} \sum g_{i j}$. Thus, their linearized dynamics are decoupled from those of the inactive modes $B_{j}$. The phases are given by

$$
\theta_{i}(t)=\phi_{\infty}-\phi_{i}^{0}+\operatorname{sign}\left(\phi_{i}^{0}-\phi_{\infty}\right) 2 \arctan \left(\exp \left(-\delta r\left|z_{\infty}\right| t+c_{1}\right)\right)
$$

with

$$
c_{1}=\ln \left(\tan \left(\frac{\left|\phi_{\infty}-\phi^{0}\right|}{2}\right)\right)
$$

showing that they are driven towards the ICMS phase $\phi_{\infty}$. The dynamics of the amplitudes $a_{i}$ is according to Eq. (7.22) governed by an inhomogeneous system of linear ODEs. The solution is a superposition of the general solution of the homogeneous system and a particular solution of the full system. Since $g_{i j}=g$ and $g_{i i}=1$, the homogeneous system has two different growth rates, both stable. The fast rate is given by $\lambda_{f}=\mathcal{A}^{2}(1+(n-1) g)$, the slow one by $\lambda_{s}=\mathcal{A}^{2}(1-g)=\mathcal{A}^{2} \epsilon$ with $\epsilon=1-g \ll 1$. A particular solution to (7.22) is obtained from

$$
\mathbf{a}=\frac{1}{2 \mathcal{A}^{2}} \mathbf{G}^{-1} \cdot \mathbf{s}
$$

where $\mathbf{G}=\left(g_{i j}\right)$ and $s_{i}=\delta r\left|z_{\infty}\right| \cos \left(\phi_{\infty}-\phi_{i}\right)$. The ansatz that $\mathbf{G}^{-1}$ is of the same form as $\mathbf{G}$ with $c$ on the diagonal and $d$ on the off-diagonals leads to

$$
\begin{aligned}
c+(n-1) d g & =1 \\
d+g(n-1)(c+(n-2) d) & =0
\end{aligned}
$$

from which follows

$$
\begin{aligned}
& \left(\mathbf{G}^{-1}\right)_{i i}=\frac{1+(n-2) g}{(1-g)(1+g(n-1)} \\
& \left(\mathbf{G}^{-1}\right)_{i j}=-\frac{g}{(1-g)(1+g(n-1)}
\end{aligned}
$$

for the entries of the inverse matrix of $\mathbf{G}$. The components of the particular solution read

$$
\begin{aligned}
a_{i} & =\delta r\left|z_{\infty}\right| \frac{(1+g(n-2)) \cos \left(\phi_{\infty}-\phi_{i}\right)-g \sum_{j \neq i} \cos \left(\phi_{\infty}-\phi_{j}\right)}{2 \mathcal{A}^{2}(1-g)(1+g(n-1))} \\
& =\delta\left|z_{\infty}\right| \frac{(1+g(n-2)) \cos \left(\phi_{\infty}-\phi_{i}\right)-\left|z_{\infty}\right| / \mathcal{A}}{2 \epsilon}
\end{aligned}
$$


where we used $\mathcal{A}^{2}=r / \sum g_{i j}$ and Eq. (7.15). Thus, the dynamics of amplitudes is decoupled in linear approximation. The particular solution (7.28) represents the amount the original amplitude is shifted under ICMS.

Consider an original amplitude $A_{i}$ with phase $\phi_{i}=\phi_{\infty}+\pi$, opposite to the ICMS phase. The ICMS strength $\delta^{*}$ suppressing the amplitude completely can be estimated from the condition $a_{i}=-\mathcal{A}$ as

$$
\delta^{*}=\frac{2 \epsilon}{\sqrt{n}(1+\sqrt{n}+g(n-2))}
$$

where we substituted $\left|z_{\infty}\right|$ by its average value

$$
\left\langle\left|z_{\infty}\right|\right\rangle_{\mathbf{x}}=\sqrt{\frac{n r}{1+(n-1) g}} .
$$

The temporal scale on which the dynamics relaxes towards the new state is

$$
t_{s}=\frac{1+g(n-1)}{\epsilon} \frac{1}{r}
$$

An ICMS with strength $\delta^{*}$ and duration $t_{S}^{*}=t_{s} r$ fulfills

$$
\delta^{*} t_{S}^{*}=\frac{2+2 g(n-1)}{\sqrt{n}(1+\sqrt{n}+g(n-2))} .
$$

In particular, for the parameter values used in Figs. 7.8 and 7.12 $g=0.98, n=8$, Eq. (7.32) predicts $\delta t_{S} \approx 0.6$ which agrees well with the estimated value of $\delta t_{S} \approx 1$.

This analysis also predicts the minimal magnitude necessary to induce a persistent change. The presence of a minimal magnitude explains the deviation from $\delta t_{S} \approx 1$ in the small $\delta$ regime. Fig. 7.12 shows that this deviations occurs at $\delta \approx 10^{-3}$. Following Eq. 7.29 the minimal strength to achieve the necessary amplitude suppression is on average $\delta_{\min }=1.5 \cdot 10^{-3}$. At very susceptible locations this value can be reduced by a factor of $\approx 2$ depending on $\left|z_{\infty}\left(\mathbf{x}_{S}\right)\right|$.

Following Eq. (7.31) the temporal scale on which the dynamics including ICMS relaxes towards the attractor is $t_{s}=3.9 \cdot 10^{2} / r$ for the set of parameters used in Fig. 7.12. This accounts for the finding that at least for $t_{S}>10^{3}$ the modification $\Delta$ and to some extent also the predictability $\mathcal{P}$ remain constant with increasing duration $t_{S}$.

To what extend does the modification $\Delta$ and the predictability $\mathcal{P}$ depend on the initial solution $z_{\infty}$ ? The linear response analysis is independent of the configuration $l=\left(l_{0}, l_{1}, \ldots, l_{n-1}\right)$ of active modes suggesting that both quantities are similar for an anisotropic attractor. Indeed, fig. 7.13 shows that both the predictability $\mathcal{P}$ and the mean modification $\Delta$ are very similar for a map with an anisotropic spectrum. 
a

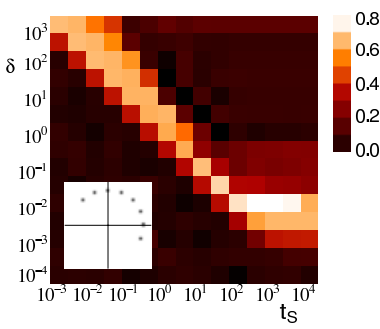

b

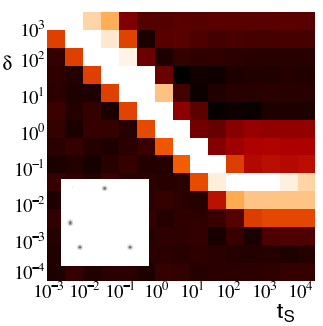

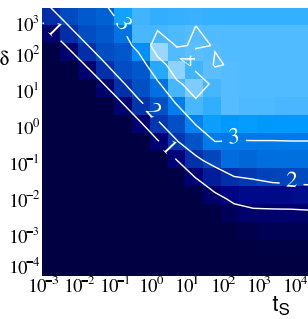

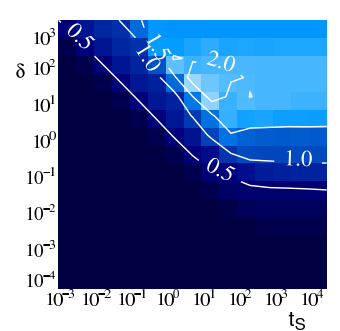

C

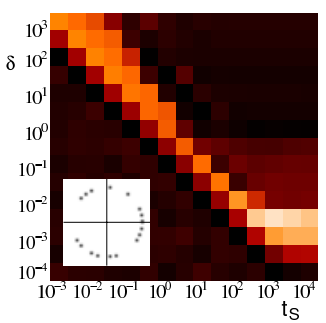

d

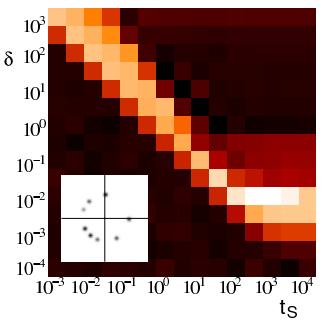

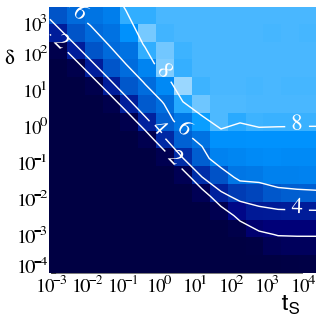

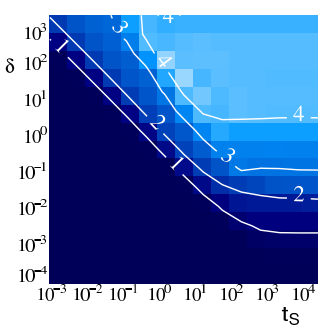

Figure 7.13: Predictability $\mathcal{P}$ (left, in red scales) and modification $\Delta$ (right, in blue scales) for a map with anisotropic spectrum containing $n=8$ active modes (a), a map with $n=9$ active modes (b), $n=17$ modes (c), and for an attractor of the full dynamics integrated numerically to $t=10^{6}$ (from Fig. 7.4 (d). Predictions of the linear response analysis are $\delta_{\min }=1.5 \cdot 10^{-3}$, $t_{S}=3.9 \cdot 10^{2}(\mathbf{a}), \delta_{\min }=4.0 \cdot 10^{-3}, t_{S}=2.0 \cdot 10^{2}(\mathbf{b})$, and $\delta_{\min }=4.9 \cdot 10^{-4}, t_{S}=8.3 \cdot 10^{2}(\mathbf{c})$. Note that both the predictability and the modification depend only weakly on the type of map.

Both the minimal strength $\delta_{\min }$ necessary to induce any persistent effect and the relevant time scale $t_{s}$ depend on the number of active modes $n$. The diagrams for a solution with $n=4$ modes (Fig. 7.13 $\mathrm{b}$ ) indicate a reduction of $t_{s}$ and an increase in $\delta_{\min }$ as predicted from Eq. (7.31) and Eq. (7.29), respectively. Likewise, for an attractor with $n=17$ modes (Fig. 7.13k) $t_{s}$ was larger and $\delta_{\text {min }}$ reduced compared to a solution with $n=8$ modes. Generally, the maximal mean modification $\Delta$ was half the number of active modes and a prediction from the softness $\kappa$ was possible in the parameter regime of moderate modification.

\subsubsection{Predicting the full dynamics}

Next we asked to what extent the reorganization $\Delta$ of the full dynamics Eq. (7.1) is predicted from the phase condition (7.16). Fig. 7.14a shows the map of modification $\Delta\left(\mathbf{x}_{S}\right)$ (reproduced from Fig. $7.4 \mathrm{p}$ ) and the map of softness $\kappa\left(\mathbf{x}_{S}\right)$ (Fig. $7.14 \mathrm{~b})$ calculated at corresponding cortical locations. The softness $k$ reproduced many characteristics of the modification $\Delta$ including variation on small scales with nearby locations often having very different values, and variation on scales large compared to the typical scale of the column pattern. Statistically, both quantities were related (Fig. 7.14 c, $C=0.40$ ). A large ICMS effect was obtained only at sites with large softness $\kappa$. At locations with small softness $\kappa$ ICMS did not cause any persistent changes in the layout of the map. Thus, the softness accurately predicts 

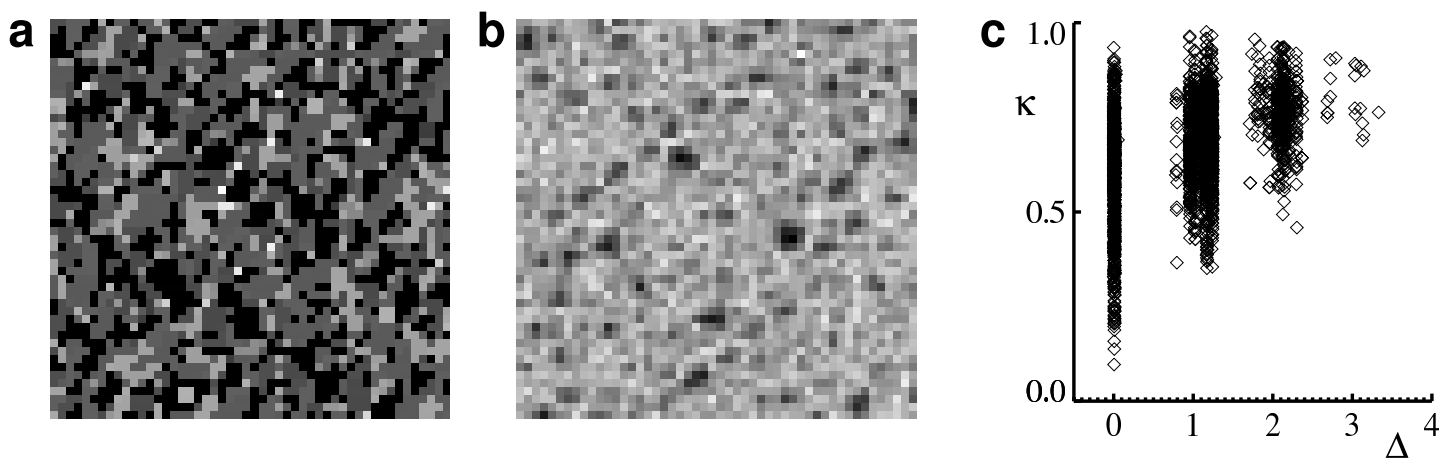

Figure 7.14: Predicting potential soft spots. a, Modification $\Delta$ from Fig. 7.4p. b, Softness $\kappa$ coded in gray scale. Bright (dark) regions mark low (high) values of $\kappa$. Note that the softness $\kappa$ shares structural features of the modification $\Delta$. c, Scatter plot $\kappa$ vs. $\Delta$ for all analyzed locations $\mathbf{x}_{0}$. The modification $\Delta$ is large only where the softness $K$ is large, i.e. a large value of $K$ is a necessary condition for significant reorganization.

the 'hard' spots, where the induction of persistent reorganization is impossible. A large value of softness, instead, is a necessary, but not a sufficient condition for inducing significant reorganization.

Why does the softness fail to provide a sufficient condition? At least partially, this is due to a considerable variation in the amplitudes $A_{j}$ of active modes shown in Fig. 7.15a (orange crosses). Generally, modes with smaller amplitudes were suppressed more severely. However, this was not simply due to their smaller size, but rather due to a reduction in stability as indicated by the stronger reduction they experience under ICMS. Fig. 7.15b shows that amplitudes with small modulus are much more often subject to switching than amplitudes with initially larger modulus. In fact, two of the larger amplitudes experienced no switching at all. One of the larger amplitudes (no. 5) often encountered successful suppression, despite its relatively small change immediately after ICMS. Fig. $7.15 \mathrm{~b}$ furthermore shows that the active amplitudes often altered their magnitude through ICMS. Apparently, the range of possible changes was discrete. An explanation for this phenomenon is unclear at present, and could include the loss of degeneration of amplitudes for large $r$ or a shifting of grain boundaries induced by ICMS. A thorough analysis of these phenomenom would be promising but requires also $5^{\text {th }}$ order contributions to the amplitude equations or to allow for spatial variation of amplitudes (envelope equations) which is beyond the scope of this work.

The softness $k$ can be refined by incorporating the dependency of the modification on amplitude differences into its definition. Crucial is a different treatment of amplitudes with phases opposed to the ICMS phase. A simple way to do this is to 

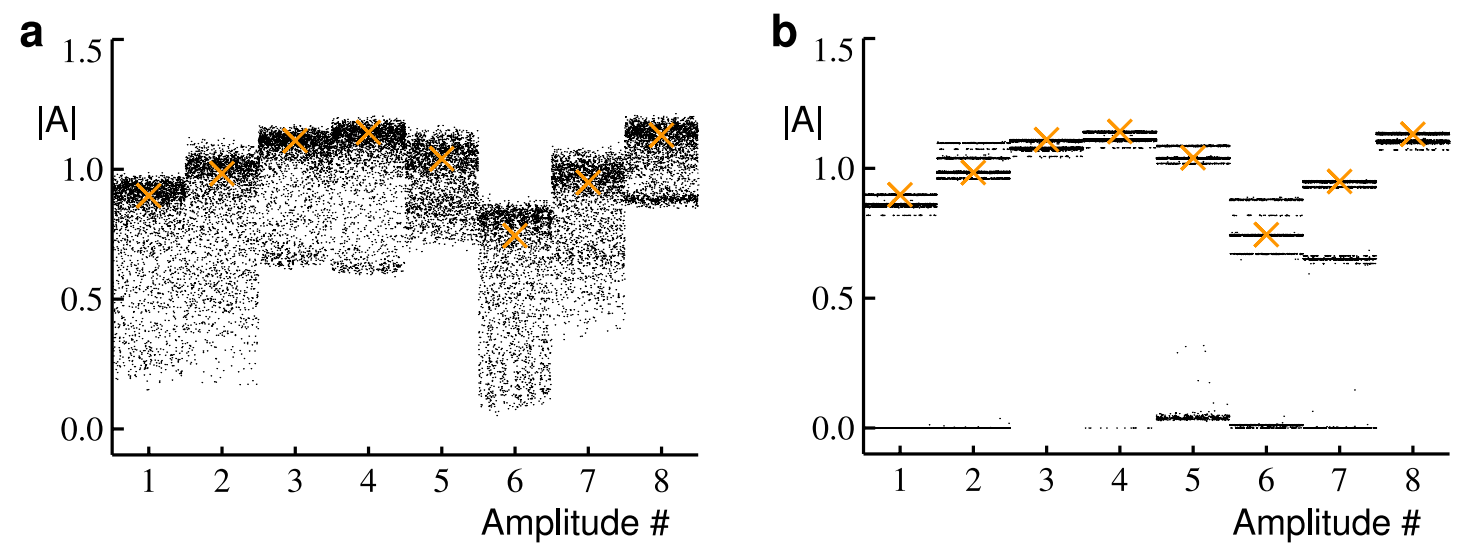

Figure 7.15: Modes with small amplitudes switch more likely. a, Absolute values $\mathcal{A}$ of the initially active amplitudes in the map from Fig. $7.4 \mathrm{a}$ (orange crosses) and their values immediately after ICMS applied at the $50 \times 50$ sites $\mathbf{x}_{S}$ shown in Fig. $7.4 \mathrm{p}$ (black dots). b, Amplitudes from a (orange crosses) after relaxation from ICMS (black dots). Amplitudes can either decay and switch with new amplitudes or alter their magnitude discretely. Note that modes with small amplitudes are stronger suppressed by ICMS and experience with higher probability complete suppression after relaxation.

c

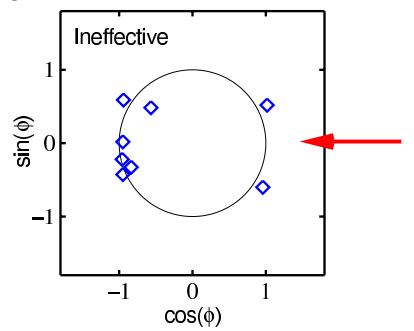

d

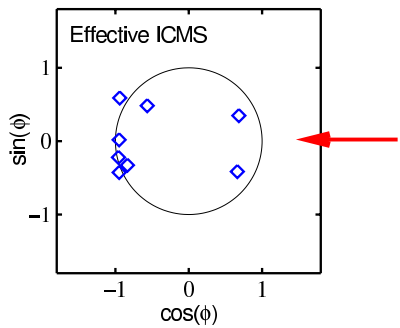

Figure 7.16: Contribution of amplitude differences to modification. a, Constellation of amplitudes $A_{j}$ (normalized to $\left\langle\mathcal{A}_{j}\right\rangle_{j}=1$ ) at site $\mathbf{x}_{S}$ with large softness $\kappa$ but small modification $\Delta$ (compare Fig. 7.14k). Illustration as in Fig. 7.10, b, Configuration at site with large $\kappa$ and $\Delta$.

define the softness by the SD

$$
\kappa^{\prime}=S D\left(\mathcal{A}_{j}^{\prime} \cos \left(\phi_{j}-\phi_{\infty}\right)\right)
$$

where

$$
\begin{aligned}
& \mathcal{A}_{j}^{\prime}=\mathcal{A}_{j}^{-q}, \quad \cos \left(\phi_{j}-\phi_{\infty}\right)>0 \\
& \mathcal{A}_{j}^{\prime}=\mathcal{A}_{j}, \quad \cos \left(\phi_{j}-\phi_{\infty}\right) \leq 0
\end{aligned}
$$

ensuring for $q>0$ and a prevalent contribution of amplitudes with smaller absolute values $\mathcal{A}_{j}$ if their phases $\phi_{j}$ point in opposite direction to the ICMS phase $\phi_{\infty}$. The softness $\kappa^{\prime}$ is small either if no amplitude exhibits a phase opposite to the ICMS phase (as in the case of $\kappa$ ), or if such amplitudes exhibit large moduli (Fig. 7.16). Only if both conditions are satisfied (as in Fig. $7.16 \mathrm{p}$ ) the softness $\kappa^{\prime}$ becomes large. 

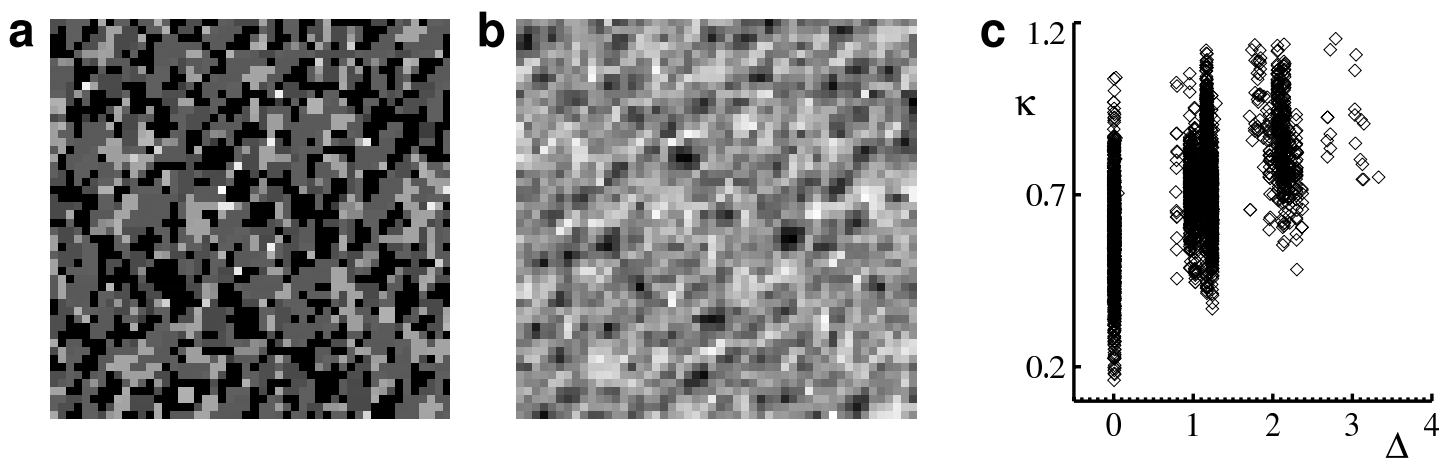

Figure 7.17: Improved prediction of potential soft spots. a, Modification $\Delta$ from Fig. 7.4p. b, Softness $\kappa^{\prime}$ coded in gray scales. Bright (dark) regions mark low (high) values of $\kappa^{\prime}$. Note that the softness $\kappa^{\prime}$ shares more structural features of the modification $\Delta$ as $\kappa$ (Fig. 7.14. c, Scatter plot $\kappa^{\prime}$ vs. $\Delta$ for all analyzed locations $\mathbf{x}_{S}$. As in the case of $\kappa$, the modification $\Delta$ is large only where the softness $\kappa^{\prime}$ is large. In addition, a large value of $\kappa^{\prime}$ implies a large $\Delta$. Thus, a large value of $K$ is a necessary and sufficient condition for significant reorganization.

For the full dynamics the softness $\kappa^{\prime}$ improves the predictability $\mathcal{P}$ of modification $\Delta$. Fig. 7.17a, b compares both quantities for the map from Fig. 7.4a. Not only appear the dark regions (low values) to overlap, as in the case for $\kappa$, but now also the bright regions (high values). Both quantities were considerably correlated as shown by the scatter plot in Fig. 7.17k $(C=0.56)$. In contrast to Fig. 7.14k, most sites with large softness showed indeed significant modification. Even higher predictabilities could be obtained by e.g. completely eliminating the contribution of amplitudes with large magnitude and phase opposite to ICMS. However, since the generalization to solutions obtained with other parameter and different dynamics is not clear, this shall not pursued further. This shows that by incorporating also the magnitude of amplitudes into the definition of the softness, as in Eqs. (7.33) with (7.34), even larger predictabilities can be attained, at least for the analyzed model.

\subsection{Discussion}

We explored ICMS in a dynamical model of the reorganization of the orientation map. We found that local stimulation can induce both transient and persistent changes dependent on the stimulation site. In general, the dynamical rearrangement continued after termination of local stimulation. ICMS induced pinwheel motion including stimulation-driven generation and annihilation of pinwheel pairs. The degree of reorganization showed a complex and sensitive dependence on the location of the ICMS site that was relatively robust against variation of model parameters leading to the notion of soft spots in the map at which ICMS induces 
spatially widespread and persistent reorganization. Since in a large and relevant parameter regime, the location of soft spots depends only little on the parameters of the model but on the layout of the map, it was possible to predict the reorganization to a substantial degree from the layout of the map.

In the analyzed model, soft spots are a consequence of the aperiodic structure of the orientation map and the property of multistability by mode competition. Inducing large reorganization by ICMS translates into a switching between different attractors which in turn requires switching among active and inactive modes. This can be achieved by suppressing a fraction of the active modes such that inactive modes can grow and finally prevail over the formerly active ones. Due to the enforcing nature of ICMS, a suppression of several active modes is realized only at particular locations in the map. The stimulation in Fourier representation acts with a phase given by the preferred orientation at the ICMS site. Since it is the preferred orientation, most Fourier components in the map exhibit a similar phase and are therefore enforced by this stimulation. However, if a fraction of modes exhibits an opposite phase, it will be suppressed by such a stimulation. Thus, following this interpretation, soft spots are at least partially determined by the phase of the stimulation relative to the phases of the active modes and therefore by the location of the ICMS site in the map.

This interpretation was formalized by the softness $\kappa$ which quantifies the efficiency of a stimulation in suppressing active modes based on the map before ICMS. For the model, the softness enables to predict the ICMS sites where no significant reorganization is induced. Thus, it identifies the ICMS sites of potentially high impact. This has interesting consequences for the experiment circumventing two difficulties a prediction of soft spots during an experiment based on a dynamical model would encounter. First, an analysis of soft spots based on this model requires many hours of computation time, even on a modern computer cluster (for instance, Fig. 7.4 required 50 days of computation on a Pentium 4 processor). Thus, without considerable improvements of numerical methods or computer hardware, it appears impossible to predict the soft spots online, during an ICMS experiment. The softness, in contrast, can be calculated from a map within minutes since it is based on the layout of the map, in fact, the only specific quantity being measured before an ICMS experiment. Second, the softness is more general than the model. Its applicability requires multistability by mode competition for solutions with multiple active modes. This property is not necessarily restricted to the class of models with permutation symmetry albeit different classes fulfilling this requirement are not known at present. 
7 Soft spots in the functional architecture of the visual cortex 


\section{Reorganization of visual cortical orientation maps by intracortical microstimulation}

\subsection{Introduction}

In this chapter we study the effects of ICMS on the orientation map in the visual cortex. The theoretical analysis of ICMS induced reorganization in terms of an attractor dynamics in Chapter 7 has exposed three primary predictions which shall be tested in the following. First, it states that an induced reorganization by ICMS can be persistent, simply as a consequence of the presence of multiple attractors. Second, it predicts that the induced effect is highly variable due to the strong dependence of the location of the ICMS site in the map. A third prediction of the analysis is that ICMS sites of potentially large modification can be predicted from the layout of the map.

To test this, we use two experimental paradigms (Fig. 8.1). In a first set of experiments, we combine ICMS with chronic experiments to test whether in the adult visual cortex an orientation map can be converted to a different map that is stable. ICMS, albeit activating a localized group of cortical neurons, is due to strong intracortical connectivity a means of inducing large scale reorganization [35]. Chronic experiments over a period of days or weeks enable to study the stable states of the orientation map provided that all relevant temporal scales of the dynamics or cortical reorganization are smaller. Thus, chronic experiments hold the promise to study the phenomenon of switching between different possible solution of the visual cortical learning dynamics.

In a second set of experiments, we conduct a series of acute experiments in order to reveal a better understanding of the reorganization processes initiated by ICMS. In these experiments ICMS is applied the same way as in the chronic experiments, but with several repetitions of recordings of the map immediately after ICMS. The temporal resolution of $\approx 2 \mathrm{~h}$ obtained with this paradigm provides an complementary view on the process of reorganization. This is important, since it is not known yet on which temporal scale cortical reorganization can take place. Furthermore, in order to identify the spreading of the induced changes we use a recording window twice as large as for the chronic recordings.

To compare the experimental results to those from the model, we have to apply 
a

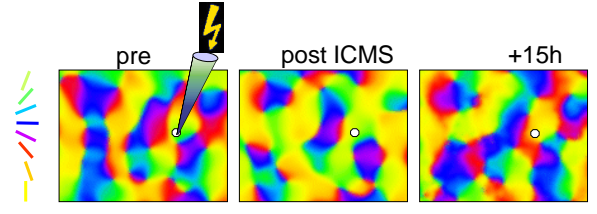

b

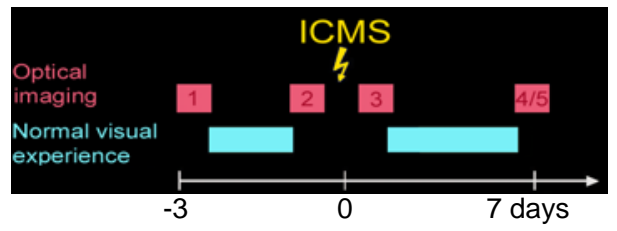

Figure 8.1: ICMS experiments. ICMS consists of 13 charged-balanced negative pulses, $6 \mu \mathrm{A}, 200 \mu$ s with $300 \mathrm{~Hz}$, at $1 \mathrm{~Hz}$ applied for $3 \mathrm{~h}$. a, Acute experiment. The orientation map is recorded two times before (Pre) and after ICMS (Post) followed by repeated measurements up to $\approx 15 \mathrm{~h}$. b, Chronic experiment. Recordings at -3 days (control), before (pre), after (post), and +7 days after ICMS. Between -3days and post, and post and +7 days the animal has normal experience.

a similar set of quantitative analyses to the ICMS data. For this, we adopt methods developed in Chapters 5 and 7 such as the quantification of similarity among maps, the pinwheel dynamics and the concept of softness. In addition to the demands for an analysis of the model, data requires preprocessing in order to diminish the influence of noise and to bring maps from a series of recordings into the same reference frame. For analyzing pinwheel densities and movement, we will use the methods developed in Chapter 4 . Experiments were conceived and designed together with Siegrid Löwel, and Hubert Dinse. The chronic experiments were conducted by Karl-Friedrich Schmidt in the lab of Siegrid Löwel at the Institute for Neurobiology (IfN), Magdeburg. Acute experiments were carried out by Klaus Kreikemeier in the lab of Hubert Dinse, Ruhr-University, Bochum. These studies are part of the PhD-thesis of Klaus Kreikemeier at the Ruhr-University, Bochum.

In this chapter, after describing the analysis methods, we study transient and persistent reorganization of orientation maps induced by ICMS. We analyze the spatial-temporal spreading of changes, the induced dynamics of pinwheels and compare the observed modification to the prediction of the softness indicator function. We find that ICMS induces pinwheel movement. Moreover, ICMS can restructure orientation maps persistently and modify their pinwheel density. The effect is highly varying and a preliminary analysis suggests that the softness might indeed predict ICMS sites with high potential for inducing large reorganization.

\subsection{Experimental paradigm and analysis methods}

\subsubsection{Alignment of maps}

Typically, the frame of reference of recordings slightly varies during an experiment. In order to compare the maps from the different recordings, their cortical coordinates must be aligned. To achieve this, we morphed all maps onto a reference map 
chosen to be the map recorded immediately before ICMS (Pre map). We used a linear morphing

$$
z(\mathbf{x})=z\left(\mathbf{x}_{0}+b \Omega_{\alpha} \mathbf{x}^{\prime}\right)
$$

with the 2-dimensional rotation matrix $\Omega_{\alpha}$, a scaling factor $b \approx 1$, and the translation $\mathbf{x}_{0}=\left(x_{0}, y_{0}\right)$. To find the 4 morphing parameters for a given map we used two methods. The first method aims to maximize the cross-correlation between maps, the second to align the position of radial vessels in images from the cortex.

The correlation method is applicable only if the map remains sufficiently similar during an experiment. For instance, if the changes induced by ICMS are too large, an alignment based on the structure of the map may become questionable. Practically, correlation coefficients down to 0.3 were tractable. For efficiency of parameter search, we used three steps of successive refinement. First, a relative wide parameter region of $\alpha=0 \pm 4.8^{\circ}, b=1 \pm 0.05, x_{0}=0 \pm 0.3 \mathrm{~mm}, y_{0}=0 \pm 0.3 \mathrm{~mm}$ was scanned using maps subsampled by a factor of 4 . For $1.6 \cdot 10^{4}$ randomly chosen parameter combinations equally distributed within these intervals, we calculated the cross-correlation within the overlap region of both maps under the condition that the overlap was sufficiently large (we required $50 \%$ of the area of each map). The parameter combination maximizing the cross-correlation was further optimized by a gradient ascent on cross-correlation. In each subsequent step the resulting parameters were used as an initial guess. Parameter ranges were decreased by a factor of 2 , the number of tested combinations decreased by a factor of 4 while the size of the maps increased by a factor of 2 .

The method using radial vessels works independently of the structure of the map. It is based on the vasculature image showing the cortex with superficial blood vessels in the region of the map (Fig. 8.2). Whereas these vessels can move considerably between subsequent recordings, the location where they enter the cortical layers, called radial vessels, remain fixed. By visual inspection, we identified a number of radial vessels within each vasculature image. The function chosen to be minimized by morphing was the average nearest neighbor distance between radial vessel locations in the reference and the morphed map. For the sake of efficiency, we used rescaling as for the correlation based method. Though in principle the method of choice, it suffers from technical difficulties concerning the recording devices. With the present equipment in the labs were the experiments were conducted, displacements of the map relative to the vasculature image are frequently. In fact, by means of local cross-correlation displacements of more that $0.6 \mathrm{~mm}$ were observed rendering the application defective given that the map has a typical scale of $\approx 1.2 \mathrm{~mm}$.

Thus, we used the radial vessel method wherever it was possible and the correlation method in all other cases. The radial vessel method was used for k109 and $\mathrm{k} 341$, the correlation method for k060, k116, k242, k343, k119 and all acute experiments. A mix of both methods was used for k114 and k242. 

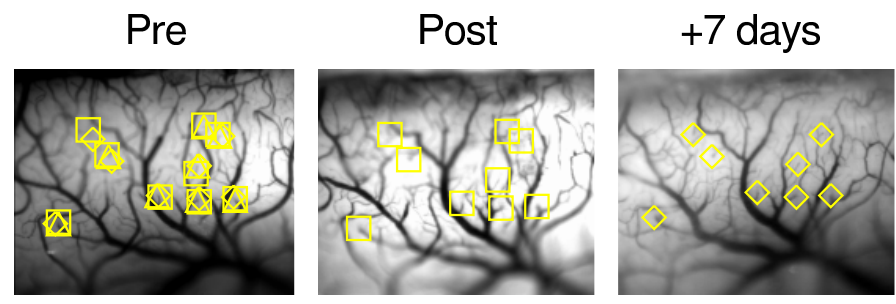

Figure 8.2: A method for ensuring for each map identical cortical coordinates with the reference map (Pre). Maps were morphed such that the radial vessels (marked by yellow symbols in each image) were aligned with those in the Pre-map (left image).

\subsubsection{Preprocessing}

After morphing all maps from a given experiment into the same coordinate system, a common region of interest (ROI) was defined excluding regions containing major vessels, boundary or reflectance artifacts, and regions with a low signal-tonoise ratio. High- and low-pass filtering was applied in the Fourier domain using a Fermi-filter as described in Chapter 4 . We used $\lambda_{h p}=1.7 \mathrm{~mm}, \lambda_{l p}=0.6 \mathrm{~mm}$, and $\beta=0.1$ for the chronic experiments (Magdeburg) in accordance with the typical column spacing of $\Lambda \approx 1.2 \mathrm{~mm}$ observed. Larger column spacings of $\Lambda \approx 1.5$ were found in the animals from the acute experiments for which we used $\lambda_{h p}=$ $2.5 \mathrm{~mm}, \lambda_{l p}=0.7 \mathrm{~mm}$.

\subsubsection{Power spectrum}

The power spectrum $|\tilde{z}(\mathbf{k})|^{2}$ of an orientation map $z$ was calculated by transforming the unfiltered map into the Fourier domain, multiplying with the high- and low-pass Fermi-filter kernels, and finally taking the squared absolute value. The resolution in $k$-space was artifically increased by a factor of 4 by zero wrapping in the spatial domain.

\subsubsection{Local similarity}

Maps of local similarity where calculated as described in Chapter 7. To reduce fluctuations over time, maps were averaged over predefined time intervals before calculating the local similarities. We used the intervals [post, 3h], [3h, 9h], [9h, 15h], [ $>15 \mathrm{~h}]$, each containing typically 2-3 maps in each experiment.

\subsubsection{Pinwheel analysis}

Pinwheels were analyzed based on the Methods developed in Chapters 4 and 5 . To estimate pinwheel locations and densities also at times in between the recordings, 
we interpolated between subsequently recorded orientation maps. To avoid further assumptions we used linear interpolation, sampled with a sufficiently large number of equally distributed maps. Usually, $m=20$ maps were sufficient for our purpose leading to a total of $m=64$ maps in the case of an experiment with recordings at 4 different times.

For each of these maps we calculated the pinwheel density using the fully automated method described in Chapter 4 using 50 low-pass cutoff wavelengths $\lambda_{l p}$ equally spaced in $[0.2,1.18] \mathrm{mm}$ with $\beta_{l p}=0.05$. Local column spacings $\Lambda(\mathbf{x})$ were calculated using wavelets with 25 scales $l_{i}$ equally spaced in the interval $d=[0.8,2.0] \mathrm{mm}$ with 12 wavelet orientations $\theta_{i}=\{0 \pi / 12, \ldots, 11 \pi / 12\}$. The calculation was performed on a grid with a spatial resolution of $0.1 \mathrm{~mm}$. Since we are primarily interested in a changing of the number of pinwheels and not of column spacing, we used for all maps the local column spacing $\Lambda(\mathbf{x})$ of the Pre map assuming that the changing of column spacing under ICMS is negligible.

For tracing individual pinwheels, we used the method described in Chapter 5. Nearest pinwheels in subsequent maps were assigned if their distance did not exceed $\Delta x=0.2 \mathrm{~mm}$. The pinwheel locations in a given (real or interpolated) map were calculated with the low-pass filtering resulting from the pinwheel density analysis. With this filtering, the low-pass cutoff wavelength $\lambda(\mathbf{x})$ generally varies for different locations. As discussed in Chapter 4 , the cutoff wavelength $\lambda(\mathbf{x})$ at a given location is constraint by the range $\left[\lambda_{0}, \lambda_{0}+\Delta \lambda\right]$ of the plateau of the filter dependent pinwheel density $\rho(\mathbf{x}, \lambda)$. For the ICMS data we used a cutoff wavelength $\lambda=\lambda_{0}+\Delta \lambda / 4$. Consistency was checked by comparing the pinwheel density in this optimally filtered maps with the pinwheel density $\rho$ from the above analysis.

\subsubsection{Softness}

To calculate the softness $\kappa_{+}$we transformed the unfiltered map into the Fourier domain and multiplied it with the Fermi filter kernels. By this procedure, low and high frequency amplitudes are eliminated. Furthermore, at low and high frequencies this also truncates the contribution from the Fourier transform of the boundary of the map (approximately varying as $\sin x / x$ over many frequencies) with which the power spectrum of the map is convolved. Neglecting the contribution of many spurious modes with small amplitudes, we truncated the sum in Eq. (8.3) by including only the $n=100$ largest amplitudes. Finally, we normalized the softness by its average value to enable a comparison between different maps.

\subsection{Persistent and transient reorganization}

To study whether orientation maps can be restructured in the adult visual cortex, we applied ICMS in a chronic experiment (Fig. 8.1p). By this, we directly test the 
primary assumption of model (5.1) namely that orientation maps are attractors of a dynamics of cortical reorganization. For such a dynamics one expects i) the possibility of switching to a different stable map and ii) the relaxation to the original map if the stimulation is not driving the map into a different basin of attraction.

\subsubsection{Persistent reorganization}

Fig. 8.3 shows the experiment k119 for which the induced reorganization was persistent over a period of 7 days. The illustration is analogous to Fig. 7.1 for the model and shows the orientation preference map $\vartheta(\mathbf{x})$, the orientation selectivity $|z(\mathbf{x})|$, the power spectrum $|\tilde{z}(\mathbf{k})|^{2}$, and the maps of local similarity $C(\mathbf{x})$ before and after a chronic ICMS experiment. As for the model, the induced reorganization was most evident in the maps of local similarity. Immediately after ICMS (Post), the induced changes were only weak and confined to the region around the ICMS site (average cross-correlation, $C=0.75$ ). Seven days later, however, the reorganization was stronger and much more widespread $(C=0.45)$ when compared to the fluctuation between the control recording before ICMS $(C=0.81)$. Interestingly, after 7 days, changes were not maximal at the ICMS site but more than $2 \mathrm{~mm}$ away from it. The orientation selectivity remained large in these regions indicating that the changes observed were not just due to noise.

In experimental maps, estimating the induced modification based on the power spectrum appears much more difficult than for the model. As in Fig. 8.3 amplitudes varied considerably in their magnitude, extension and form. Due to their relative small size the resolution of the maps in Fourier space was rather coarse. Nevertheless, in this experiment k119 a closer inspection of the two control maps recorded before ICMS revealed that the spectra shared most peaks, whereas the spectrum 7 days after ICMS apparently exhibited at approximately seven o' clock a peak that was not present before ICMS.

\subsubsection{Transient reorganization}

A very different progression was observed for experiment k116 shown in Fig. 8.4. Here, the initial map almost recovered completely 7 days after the application of ICMS $(C=0.87)$ even though immediately after ICMS, the induced changes were stronger compared to $\mathrm{k} 119$ and more widespread $(C=0.71$; similarity before ICMS (control), $C=0.89$ ). The largely complete recovery was also indicated by the similar locations of the amplitudes in the power spectra before and 7 days after ICMS. The only signature of persistent reorganization after 7days was the annihilation of a pinwheel pair at the right hand side. Thus, whereas in experiment k119 after the termination of ICMS the map progressively rearranged, in k116 it returned to its original state consistent with a relaxation-like dynamics as predicted from an 


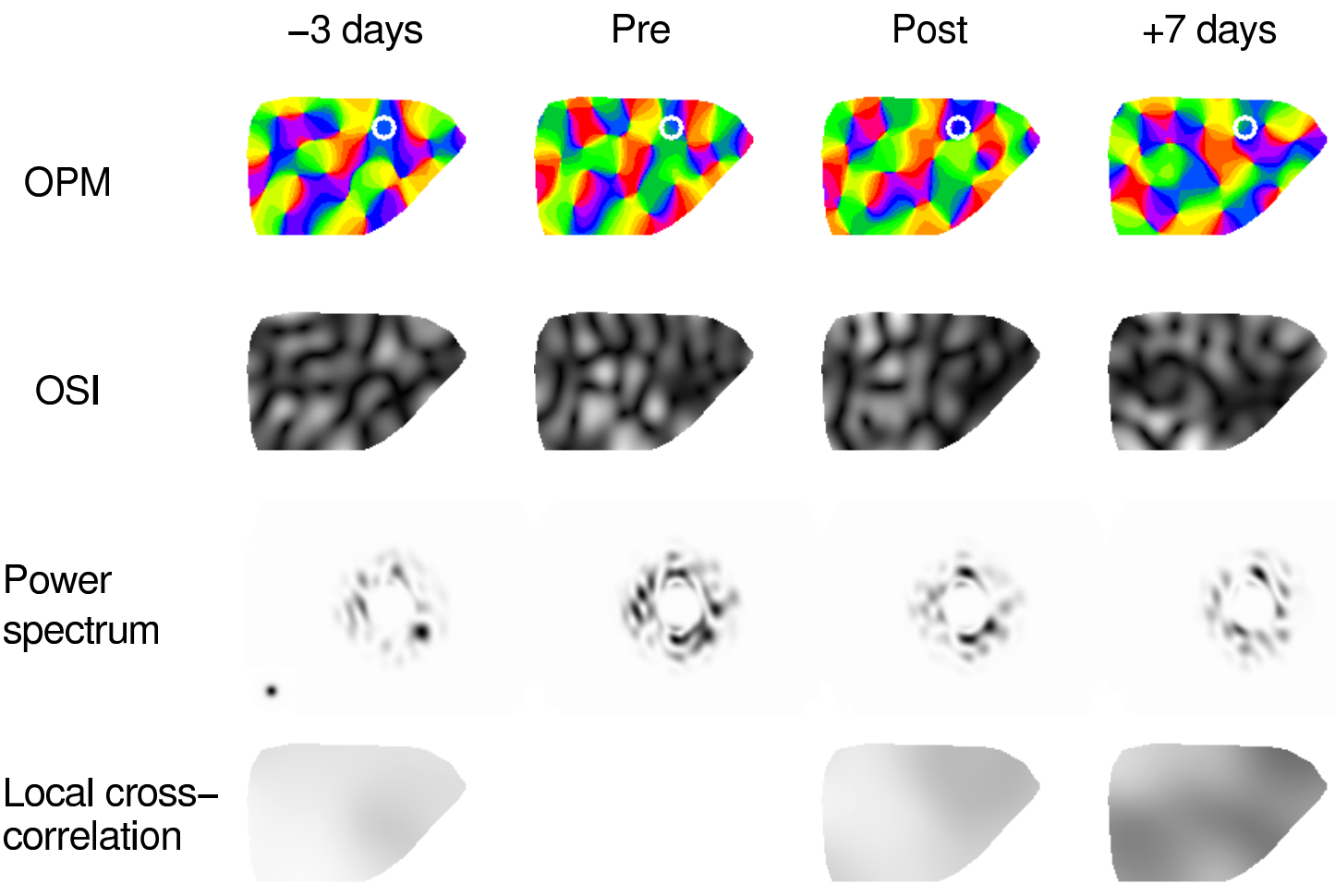

Figure 8.3: Persistent changes induced by ICMS (Experiment k119). Orientation preference map (OPM), orientation selectivity (OSI), 2D power spectrum (origin of $k$-space at center of annulus) and map of local similarity 3 days before, immediately before (Pre), immediately after (Post) and 7 days after the application of ICMS. Bright (dark) regions in the similarity maps mark larger (smaller) correlations with the pre condition. The dark spot on the lower left of the -3 days spectrum indicates the resolution in $k$-space. Immediately after ICMS, changes are confined to the vicinity of the ICMS site. After seven days changes cover even remote regions (crosscorrelation, Pre/-3days, $C=0.81$; Pre/Post, $C=0.75$; Pre/+7days, $C=0.45$ ). For comparison with the model, refer to Fig. 7.2

attractor model.

\subsubsection{Two classes of experiments}

Guided by these two cases we classified all experiments into two classes based on visual inspection of orientation maps and maps of local similarities. The first class consists of all experiments for which a widespread rearrangement was persistent over the period of 7 days. The second class contains all experiments for which the map showed a tendency to recover completely within a few days after ICMS. From the six experiments, three fitted into the first class and three into the second class. The time course of rearrangement quantified by the similarity $C$ with the reference 


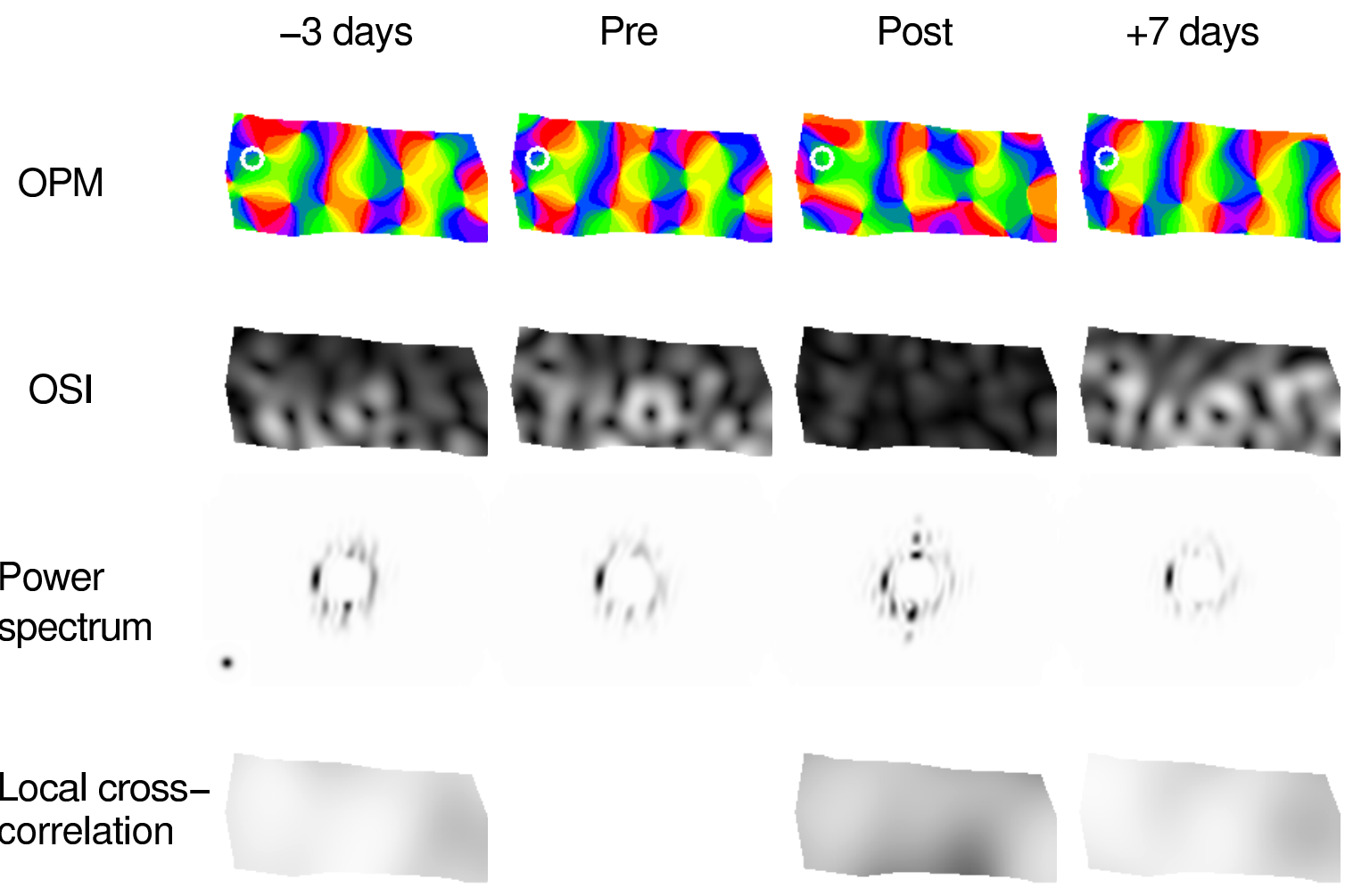

Figure 8.4: Transient changes induced by ICMS. Illustration as in Fig. 8.3. In this experiment (k116), the final map is very similar to the initial one (cross-correlation, Pre/-3days, $C=0.89$; Pre/Post, $C=0.71$; Pre/+7days, $C=0.87$ ). Immediately after ICMS the map is different even at regions far away from the ICMS site. However, apart from a pinwheel pair vanishing at the right end, the map nearly recovers after 7 days. For comparison with the model, refer to Fig. 7.2

map recorded immediately before ICMS is summarized in Fig. 8.5. Non-recovery experiments are marked in orange, recovery-experiments in blue. Grey symbols mark sham-experiments, i.e. control experiments carried out in identical fashion, but without inducing any current in the cortex. Generally the similarity was reduced immediately after ICMS when compared to the control similarity between maps recorded before ICMS. In the recovery-class, similarities almost returned to this baseline after 7 days. In experiment k114 for which the map was recorded already 3 days after ICMS, the similarity exhibited an intermediate value suggesting that the map had recovered only partially after this period. A possible interpretation is that the reorganization associated with recovery to the original state can last more than 3 days. In the non-recovery class, the similarity either remained constant on a relatively low level 5-7 days after ICMS or decreased progressively. Interestingly, this decrease was observed to initiate after 5 days in experiment k109 for which the map was recorded twice after 5 and after 7 days. Consistent with 


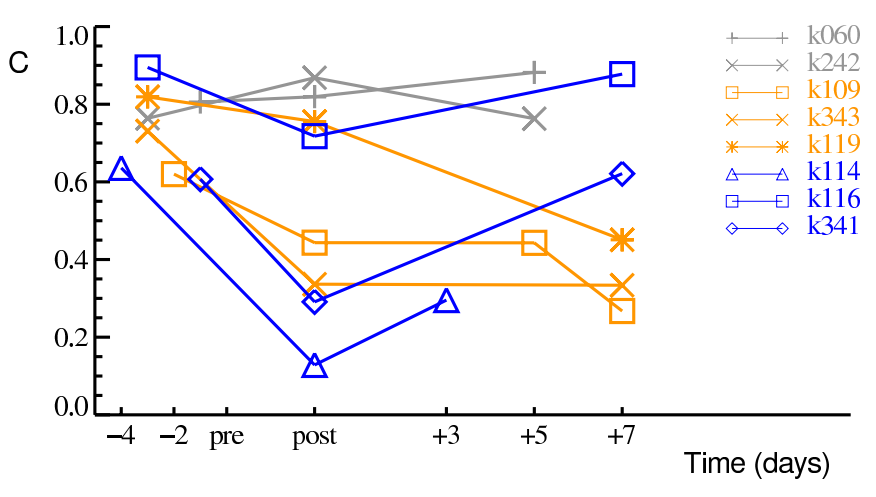

Figure 8.5: Reorganization induced by ICMS: Summary of experiments. Depicted are similarities $C$ between the reference map recorded immediately before ICMS (Pre) and the map at other stages of the experiment. Different symbols denote different animals. Grey indicates sham experiments, orange cases with persistent, blue with just transient reorganization.

experiment k114, this suggests that the process of reorganization induced by ICMS can require several days.

Following Fig. 8.5, the degree of reorganization obtained in an experiment can be quantified by comparing the similarity values $C$ at different stages of the experiment. For this purpose we defined the modification

$$
\Delta_{C}=\frac{C_{\text {post }}}{C_{\text {late }}}
$$

as the quotient between the similarity $C_{\text {post }}$ observed immediately after ICMS and the similarity $C_{\text {late }}$ obtained several days after ICMS for the latest map being recorded. The modification $\Delta_{C}$ is large if the induced reorganization is large and it is small if the map largely recovers. It will be used in Section 8.6.

We conclude that by ICMS, orientation maps can be converted to a different map persistent at least over 7 days. Moreover, induced changes can be transient leading to a largely complete recovery of the original map. Both observations support the hypothesis that the reorganization of the orientation map follows an attractor dynamics.

\subsection{Spatial-temporal dynamics}

In order to obtain a better understanding of the reorganization initiated by ICMS, we conducted a series of acute experiments (Fig. 8.1 a). Besides the relatively high temporal resolution gained with this method (of $\approx 2 \mathrm{~h}$ ) we also increased the frame of the recording window by a factor oft two since we were interested in the spreading and the spatial range of the induced changes.

We observed a progressive rearrangement in the hours following ICMS. The rearrangement developed continously indicating that the reorganization evolves with a temporal scale on the order of $1 \mathrm{~h}$ (Fig. 8.6). Largest changes were found within 
a distance of $3 \mathrm{~mm}$ from the ICMS site and often, the maximum change was not directly at the ICMS site. For the experiment shown in Fig. 8.6a, the induced changes were initially small and remained small up to $3 \mathrm{~h}$ before becoming much stronger after $9 \mathrm{~h}$. In contrast, for the experiment in Fig. 8.6p, the effect was maximal immediately after ICMS and decreased thereafter. In the example shown in Fig. 8.6. changes went back and forth. The observed increase and decrease often resembled a process of relaxation further supporting the hypothesis of an underlying attractor dynamics. However, a reorganization as strong as observed for the chronic experiments 7 days after ICMS was generally not seen in the acute experiments. One possible interpretation consistent with experiments k114 and k109 (Fig. 8.5) is that the reorganization of large regions of the map and regions away from the ICMS site takes place on the temporal scale of days rather than hours. An alternative explanation is that reorganization may require normal visual experience or sleep.
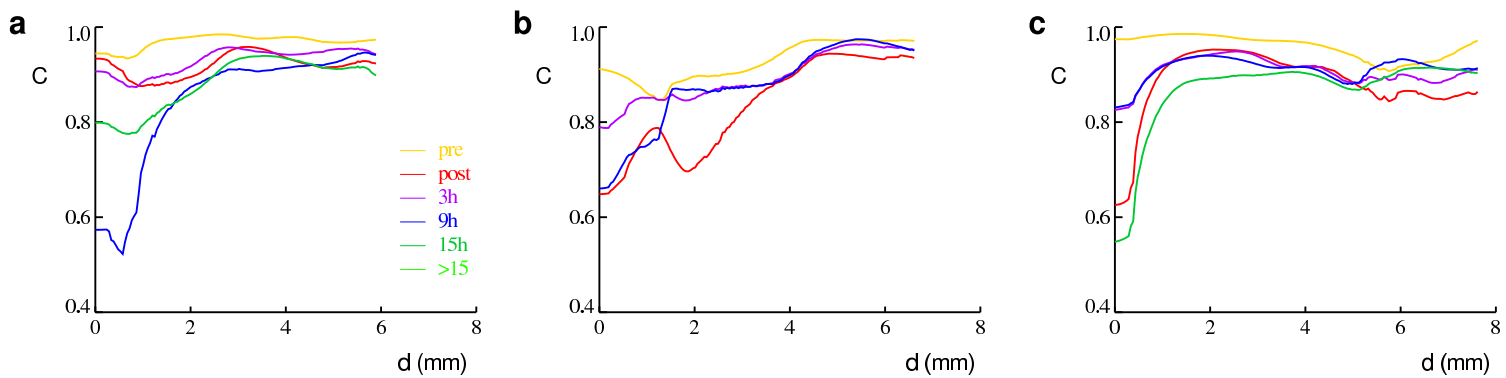

Figure 8.6: Transient changes induced by ICMS. a, b, c, Similarity $C_{0}$ as a function of the distance from the ICMS site (averaged over concentric rings around the ICMS site) evolving in acute experiments. Induced changes can increase substantially over a period of several hours after termination of ICMS (a). Already immediately after termination of ICMS (post) the induced changes can spread over $3 \mathrm{~mm}$ (b). Changes can decrease after ICMS and progressively increase at a later stage (c). For comparison with the model, refer to Fig. 7.3

\subsection{Induced pinwheel dynamics}

A further quantity of particular interest is the pinwheel density. In Section 7.5.2 we showed that the model predicts a complex pinwheel dynamics under ICMS including movement, creation and annihilation during and after ICMS. In this Section we test this by applying the pinwheel analysis developed in Chapter 4 to the ICMS data. To estimate the position of pinwheels between the recordings, we linearly interpolated between subsequent maps (see Section 8.2). 


\subsubsection{Pinwheel movement}

Pinwheels were found to move during and after the application of ICMS. Occasionally, pinwheel pairs were annihilated or new pairs were created as shown in Fig. 8.7 displaying the pinwheel maps for the two experiments from Fig. 8.3 and Fig. 8.4. Some pinwheels moved out of the observation frame or entered it from outside. The traces of pinwheels throughout the experiment are shown in Fig. 8.7p, c. In the recovery experiment $\mathrm{k} 116$, pinwheels either wiggled around or if moving under ICMS, returned along nearly the same path after ICMS (8.7p). In the nonrecovery experiment $\mathrm{k} 119$, pinwheels covered longer paths reaching larger final distances (Fig. 8.7 d). Some pinwheels continued to move after ICMS into the same direction then during ICMS. Pinwheels traveling farther than a column spacing $\Lambda \approx 1.3 \mathrm{~mm}$ were not observed.

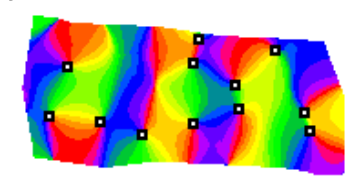

Pre

C

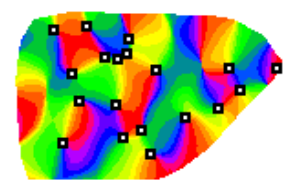

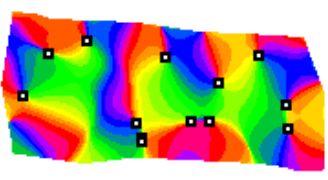

Post

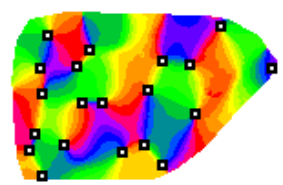

b

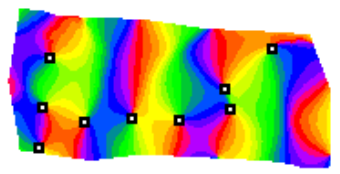

+7 days

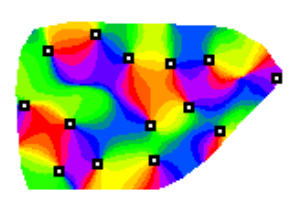

d
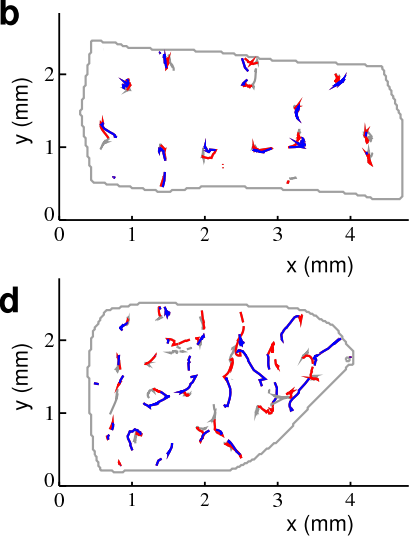

Figure 8.7: ICMS induces pinwheel dynamics. a, Pinwheel centers (marked by white dots) in the recovery experiment k116 from Fig. 8.4 b. Pinwheel traces during reorganization by linear interpolation between recorded maps. The red part of each trace marks the movement during ICMS (Pre to Post), the blue part the movement after ICMS (Post to +7 days). The grey traces mark the movement before ICMS (-3days to Pre). c, d, Pinwheel centers (c) and pinwheel traces (d) in the non-recovery experiment k119 from Fig. 8.3. Note that in the non-recovery experiment traces are longer.

\subsubsection{Pinwheel density}

By annihilation and creation of pinwheels, the pinwheel density can change over time. Indeed, the traces of pinwheel density $\rho(t)$ for the two experiments from Fig. 8.7 showed a considerable variation of pinwheel density $\rho$ over the time course of the experiment compared to the control fluctuations before ICMS (Fig. 8.8). The time course of the pinwheel density was more complex for the non-recovery map k119 showing larger fluctuations. In both maps, an initial density of $\rho \approx 2.4$ decreased to $\rho \approx 1.87$ days after ICMS. Thus, ICMS is indeed capable of inducing 
changes in the pinwheel density. These changes are not captured by the measure of similarity used e.g. in Fig. 8.5.

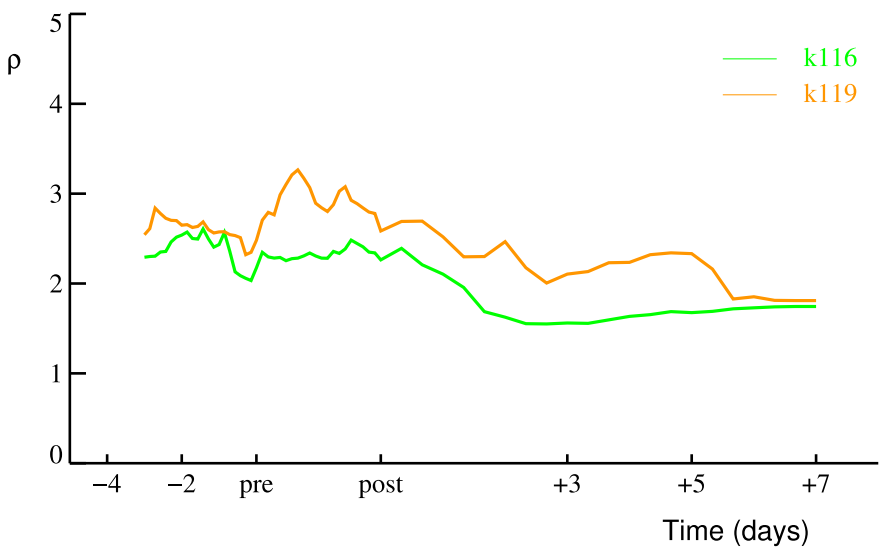

Figure 8.8: ICMS changes pinwheel density as shown by its time course $\rho(t)$ for the two experiments from Fig. 8.7. Densities fluctuate in the non-recovery map k119. In both cases, largest changes occur after ICMS and the final densities are reduced. For comparison with the model, refer to Fig. 7.5 .

\subsection{Soft spots in the visual cortex}

In Section 7.7 of the previous chapter we developed methods for predicting from a map its soft spots, i.e. the ICMS sites with potential for significant restructuring of the orientation map. As highlighted in Fig. 7.14 the softness $\kappa$ provides a necessary condition for the degree of reorganization. In this closing Section we apply this concept to the ICMS experiments in the visual cortex. First, we define the softness $\kappa_{+}$that is used for experimental maps. In a second step, we compare the predicted to the observed modification.

\subsubsection{Softness}

The softness as defined in Eq. (7.18) can in principle be calculated for an arbitrary orientation map. However, with the real maps analyzed here one is facing several problems. First, there is noise on many spatial scales modifying the active amplitudes and introducing new artificial ones. Second, the maps are relatively small implying a low resolution in the Fourier domain. The measured amplitudes may be a superposition of many nearby amplitudes. Third, the modulus of the measured amplitudes vary over orders of magnitude. This might be simply a consequence of the first two points or reflect the actual dynamics of the visual cortex. To account at least partially for these factors, we defined for the experimental maps the softness by

$$
\kappa_{+}\left(\mathbf{x}_{S}\right)=\frac{1}{N} \sum_{j}\left[\mathcal{A}_{j}\left(\phi_{j}\left(\mathbf{x}_{S}\right)-\phi_{\infty}\left(\mathbf{x}_{S}\right)\right)\right]_{+}
$$


a

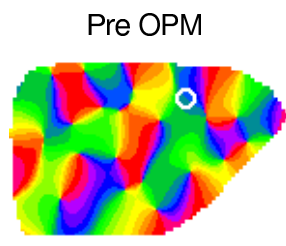

b

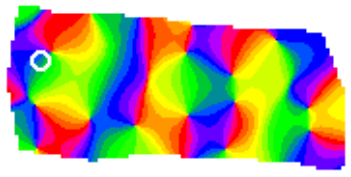

Soft spots
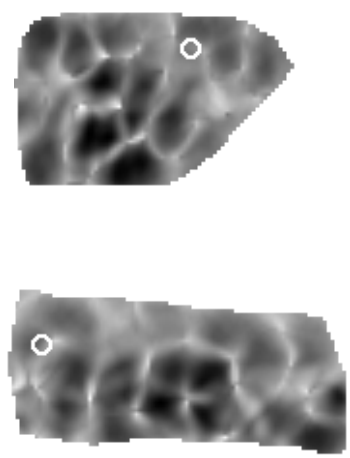

c

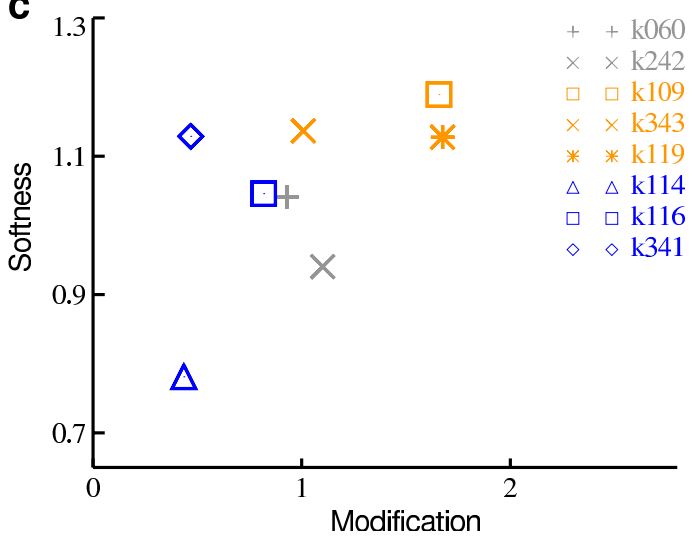

Figure 8.9: Predicting soft spots in the visual cortex. a, Orientation preference map (left) and map of softness $\kappa_{+}$(right) for the non-recovery case k119 (Fig. 8.3). Bright (dark) regions mark large (small) values. Note that the softness at the ICMS site is relatively large $\left(\kappa_{+}=1.13\right)$. $\mathbf{b}$, Orientation map and softness for the recovery case k116 (Fig. 8.4. In this case, the softness at the ICMS site is moderate $\left(\kappa_{+}=1.05\right)$. c, Softness $\kappa_{+}$at the ICMS site vs. modification $\Delta_{C}$ (Eq. (8.2) for all analyzed experiments. For comparison with the model, refer to Fig. 7.14k.

where $[x]_{+}=x$ for $x \geq 0$ and $[x]_{+}=0$ for $x<0$. The softness $\kappa_{+}$sums up all amplitude components opposed to the ICMS direction $\phi_{\infty}$. It is normalized by the average value of a map $N$ to ensure comparability between different maps. The softness $\kappa_{+}$has similar properties than the softness $\kappa$ defined in Eq. (7.18) that was used for the analysis of the model. In fact, for model orientation maps, $\kappa$ and $\kappa_{+}$ were highly correlated with similar diagrams of predictability $\mathcal{P}$ (Fig. 7.12). However, important properties of the softness $\kappa_{+}$favor its application to experimental maps rather than the softness $\kappa$. First, in $\kappa_{+}$, each amplitude is weighted by its modulus to avoid the prevalent contribution of small amplitudes to the softness. This is necessary since for experimental maps the measured active amplitudes generally accumulate around $\mathcal{A}=0$. These amplitudes may be due to noise and even if real, a switching would barely lead to significant rearrangement of the maps. Second, the softness $K_{+}$neglects the contribution of amplitudes with phases pointing towards the ICMS phase. It is large only if many large amplitudes are opposed to the ICMS direction. Since amplitudes accumulate in the vicinity of $\mathcal{A}=0$, the $\mathrm{SD}$ of $\cos \left(\phi_{j}-\phi_{\infty}\right)$ does not separate well favorable from unfavorable phase constellations. In fact, $\kappa$ is relatively large also if most larger amplitudes are pointing to the direction of ICMS which, however, implies a low potential for reorganization.

\subsubsection{Predicting soft spots in the visual cortex}

Fig. 8.9 shows the maps of softness $\kappa_{+}$for the two experiments $\mathrm{k} 119$ and $\mathrm{k} 116$ from Fig. 8.3 and 8.4 respectively. As observed for the model, the softness varied on 
spatial scales larger and smaller than the orientation map itself. In some regions, nearby points exhibited very different values whereas in other regions, large or small values were predominant. Whereas in the non-recovery experiment $\mathrm{k} 119$, the softness was with $\kappa_{+}=1.13$ relatively large at the ICMS site (Fig. 8.9a), it was with $\kappa_{+}=1.05$ only moderate in the recovery experiment k116 (Fig. 8.9p).

Fig. 8.9k summarizes the values of softness $\kappa_{+}$from all experiments and shows its relation to the actual observed modification $\Delta_{C}$. Large modifications $\Delta_{C}$ were only observed in cases where the softness $\kappa_{+}$was large at the ICMS site. At least for the present data, a small softness accompanied the absence of persistent reorganization. This resembles the results obtained for the model (compare Fig. 7.14c) suggesting that also for the visual cortical orientation map a large softness is a necessary condition for inducing significant and persistent reorganization.

\subsection{Discussion}

In this chapter, we showed that ICMS is capable of inducing substantial reorganization of the visual cortical orientation map. While initially confined to the vicinity of the ICMS site, induced changes evolved on temporal scales between hours and days to more remote regions of the map. The outcome of experiments was highly variable. In one class of experiments, the initial map largely recovered completely. In a second approximately equally large class, changes were persistent at least over a period of 7 days suggesting that a new stable map has formed.

The reorganization was accompanied by pinwheel movement, and annihilation and creation processes. The density of pinwheels was found to change considerably during and after the application of ICMS and to differ significantly in the initial and final map. This was true for both classes of experiments indicating that pinwheel densities can change strongly by only slightly changing the map and that a substantially modified map does not imply a different pinwheel density necessarily. We observed a tendency of ICMS to enforce a more stripe-like columnar organization thereby decreasing the density of pinwheels.

Within the theoretical framework developed in Chapter 7 these experimentally observed effects of ICMS are interpreted as a switching between several different equilibrium states of a cortical learning dynamics. This interpretation suggests that the observed variability in the persistence of the induced effect is due to its sensitive dependence on the location of the ICMS site in the map. To test this, we compared the predicted modifiability associated with a given ICMS site quantified by its softness to the actually observed modification. We found that this relation strongly resembled the analog relation for the model suggesting that also for the visual cortex, a large softness at the ICMS site is a necessary condition for inducing persistent modification implying that potential ICMS sites can be predicted from the layout of the map only. A further corroboration of these results requiring more 
experiments appears necessary. However, in upcoming studies this prediction can be tested much more specifically, since due to its simplicity the softness can be calculated online during an experiment enabling the possibility of a more purposeful selection of the stimulation site. 


\section{Conclusion}

Nature uses as little as possible of anything.

(Johannes Kepler)

In this thesis, we investigated the pattern of preferred stimulus orientations in the primary visual cortex of higher mammals, known as the orientation map. Based on a pattern formation model including only key features of visual cortical organization, we analyzed the dynamics of the orientation map during normal development and under perturbation. In particular, we focused on the statistics and dynamics of abundant topological defects in orientation maps, called pinwheels. The theoretical analysis was complemented by a comprehensive quantification of experimental data. This revealed several interesting observations that were often quantitatively reproduced by the model.

Analyzing pinwheel densities in data from the visual cortex, we found that in diverse species such as ferrets (carnivora), tree shrews (scandentia), and galagos (primate), orientation maps share several universal properties: Pinwheels occur with equal overall density, similar spatial variation and virtually identical distributions of nearest neighbor distances. The pattern formation model studied, a generalized Swift-Hohenberg equation with multiple attractors, quantitatively reproduces the observed universal properties of orientation maps in the apparently realistic parameter regime where long-range interactions between remote contour detectors are present. In the model, the observed pinwheel density is robustly selected briefly after the emergence of the orientation map and remains stable throughout development. Also the distributions of pinwheel nearest neighbor distances are largely preserved. Nevertheless, we proposed quantities capturing more sensitively the reorganization during map development including pinwheel annihilation and creation rates, survival fraction, pinwheel pathlengths and pinwheel speed. This shall provide means for future experiments to further corroborate or falsify the predictions of the model.

We found that similar reorganization processes can also be evoked by stimulation. Extending the model to mimic the effect of so-called intracortical microstimulation (ICMS) we studied the rearrangement of the orientation map during and after this localized stimulation. Orientation maps display widespread rearrangement including complex pinwheel dynamics. If the stimulation drives the orien- 
tation map into a new basin of attraction, the induced modification is global and persistent. The degree of modification strongly depends on the location of stimulation and is particularly high when stimulating at specific locations in the map, named soft spots. The potential locations of these soft spots are largely determined by the layout of the map. The quantity softness capturing this dependency predicts the potential soft spots of a map from its layout. The softness provides a necessary condition for inducing large modification. We tested these predictions of the model by analyzing ICMS experiments. We observed a transient reorganization often continuing after the termination of ICMS. In one group, maps relax to a near original state after 7 days. In another group the changes persist at least over a period of 7 days. In both groups, ICMS induces movement of pinwheels and a changing of their density. The variability of induced reorganization appears consistent with the hypothesis of soft spots in visual cortical orientation maps and the results indeed suggest the possibility of predicting potential soft spots from the softness. This further agreement between model and experiment supports the interpretation of the visual cortical orientation map as the result of activity-dependent self-organization. Soft spots, defined more generally as stimulations for which a network is especially susceptible, appear as a natural consequence of complex circuitry subject to a large scale reorganization principle.

Our investigation revealed two important properties of pinwheels. The first property is the as astounding as unexpected observation that the average density was not just constant, but close to the mathematical constant $\pi$. An average pinwheel density close to $\pi$ is reproduced by the model within a large and apparently realistic parameter regime. Moreover, near criticality, the pinwheel density is exactly $\pi$ in the limit of infinitely long interaction range. Because of this agreement it is natural to conjecture that the pinwheel density in the visual cortical orientation map is $\pi$. This interpretation has several important implications. First, it poses a severe constraint on any theory describing the development of orientation selectivity in the visual cortex. The model class studied in this thesis largely satisfies this requirement. However, this class may not be the only possibility. All other models proposed so far need to be reconsidered, since they do not seem to be consistent with this finding. Second, the occurrence of $\pi$ suggests the presence of a large scale optimization principle for visual cortical circuitry. That the cortex is optimized for something is widely believed. The $\pi$-principle casts a new light on the enquiry for such a principle.

A second property concerns the controversial issue of whether pinwheels are static, anatomically fixed building blocks of a prespecified cortical machinery or rather flexible entities that can change their place and interrelation dependent on patterned cortical activity. Our analysis clearly supports the latter interpretation. This was indicated by the model showing that reorganization is accompanied by pinwheel movement, and annihilation and creation processes. Such pinwheel dynamics occurred during normal development and after perturbation by ICMS. More 
importantly, this was also found in the visual cortex by an analysis of reorganization induced by ICMS. We observed induced pinwheel motion including stimulationdriven generation and annihilation of pinwheel pairs that can lead to a change of the pinwheel density in individual maps.

What are the implications of this for cortical development? Cortical development is often thought to be guided by external factors. Following one branch of hypotheses, cortical circuitry is laid out in detail under genetic control [41, 46, 79]. Other lines of arguments suggest that sensory experience instructs connectivity through some Hebbian mechanism [20, 35, 38, 62, 82, 92]. The universal pinwheel statistics reported here are hard to reconcile with either of this view. Genetic instruction, on the one hand, is unlikely to produce congeneric maps in species separated for more than 60 million years of evolution. Sensory instruction, on the other hand, if prevailing would rather counteract a universal organization given the substantial differences in their upstream visual systems in the different animals.

Our results rather indicate a predominance of emergent properties of cortical circuitry. Neocortical neurons are embedded in densely connected networks. Changing synapses driving a neuron may influence its firing pattern and by this alter its synapses to other neurons which in turn may change their activity. Hence, local changes induce further changes, finally influencing the network as a whole. Presumably, not every configuration of the network is stable under the persistent influence of cortical activity. A map self-organizes until a global stable 'synaptic ground state' is reached. The present study suggests that such synaptic ground states exist and belong to a specific class at least partially characterized by the described universal pinwheel statistics.

What is the role of activity in this process? Unless being deteriorating [103], spontaneously generated activity appears sufficient for establishing a map within the described class. In this respect, activity is only permissive and not instructive. The class is largely stable against changes of the environment, and apparently even stable against mutations during evolution. The selection of the map within this class, however, may be instructed by specific patterns of activity or other external factors. The possible range within the limitation set by the class is illustrated by the distinct systematic variation of local pinwheel densities we observed in the tree shrew compared to the ferret.

Thus, pattern selection appears beneficial as it guarantees the development of a proper map in the visual cortex. In the model studied here, this is accompanied by a considerable stability during development, a property which appears important from a physiological point of view. On the one hand, the stability of the orientation map observed in previous experiments has been interpreted as evidence that the visual cortical architecture is fixed and probably prespecified [17, 79, 81]. Selforganization models on the other hand have often been associated with massive reorganization during development. Our model study shows that both interpretations are not conclusive. We found that the reorganization of the orientation map 
decays algebraically. Although reorganization never really comes to an end, it is strongest during early development. The reorganization observed in the analyzed model is consistent with the experimental evidence for the development of the orientation map.

After all it is remarkable that our approach works at all. Even at second sight, the brain seems overwhelmingly difficult to describe. Since the pioneering work of the neuroanatomist Cajal (1852-1934), the relevant parameters seem to be hidden somewhere in the circuitry. On the one side, there is this seemingly highly elaborated and densely connected network of which each constituent itself is a highly nonlinear and spatially extended system. On the other side, there is a model consisting of a field with some effective interactions, an average over an ensemble of activity patterns, ignoring further response properties of neurons and crosstalk with other parts of the brain. The agreement between both, noted by measuring a set of cortices, offers some hope that even in the brain when described at an appropriately chosen level things may follow simple principles. 


\section{Bibliography}

[1] Albus, K. A quantitative study of the projektion area of the central and the paracentral visual field in area 17 of the cat. Exp. Brain Res. 24, 181 (1975).

[2] Angelucci, A., JB, J. L., Walton, E., Bullier, J. H. J., and Lund, J. Circuits for local and global signal integration in primary visual cortex. J. Neurosci. 22, 8633 (2002).

[3] Ball, P. The self-made tapestry: Pattern formation in nature. Oxford University Press, New York (1999).

[4] Bartfeld, E. and Grinvald, A. Relationships between orientation-preference pinwheels, cytochrome oxidase bolbs, and ocular-dominance columns in primate striate cortex. Proc. Natl. Acad. Sci. 89, 11905 (1992).

[5] Blasdel, G., Livingstone, M., and Hubel, D. Optical imaging of orientation and binocularity in visual areas 1 and 2 of squirrel monkey (samiri sciureus) cortex. J. Neuroscience 12, 1500 (1993).

[6] Blasdel, G. G. Orientation selectivity, preference, and continuity in monkey striate cortex. J. Neurosci. 12, 3139 (1992).

[7] Blasdel, G. G. and Salama, G. Voltage-sensitive dyes reveal a modular organization in monkey striate cortex. Nature 321, 579 (1986).

[8] Bodenschatz, E., Pesch, W., and Ahlers, G. Recent developments in rayleighbénard convection. Annu. Rev. Fluid Mech. 32, 709 (2000).

[9] Bonhoeffer, T. and Grinvald, A. Iso-orientation domains in cat visual cortex are arranged in pinwheel-like patterns. Nature 353, 429 (1991).

[10] Bonhoeffer, T. and Grinvald, A. The layout of iso-orientation domains in area 18 of cat visual cortex: Optical imaging reveals a pinwheel-like organization. J. Neurosci. 13, 4157 (1993).

[11] Bonhoeffer, T. and Grinvald, A. Brain Mapping: The Methods, pages 55-97. Academic, San Diego (1996). 
[12] Bonhoeffer, T., Kim, D.-S., Malonek, D., Shoham, D., and Grinvald, A. Optical imaging of the layout of functional domains in area 17 and across the area 17/18 border in cat visual cortex. Europ. J. Neurosci. 7, 1973 (1995).

[13] Bosking, W., Zhang, Y., Schofield, B., and Fitzpatrick, D. Orientation selectivity and the arrangement of horizontal connections in tree shrew striate cortex. J. Neurosci. 17, 2112 (1997).

[14] Braitenberg, V. and Braitenberg, C. Geometry of orientation columns in the visual cortex. Biol. Cybern. 33, 179 (1979).

[15] Braitenberg, V. and Schütz, A. Cortex: statistics and geometry of neuronal connectivity. Springer, Berlin (1998).

[16] Chapman, B. and Stryker, M. Development of orientation selectivity in ferret visual cortex and effects of deprivation. J. Neurosci. 13, 5251 (1993).

[17] Chapman, B., Stryker, M., and Bonhoeffer, T. Development of orientation preference maps in ferret primary visual cortex. J. Neurosci. 16, 6443 (1996).

[18] Chklovskii, D. B. and Koulakov, A. A. Maps in the brain: what can we learn from them? Annu. Rev. Neurosci. 27, 369 (2004).

[19] Constantine-Paton, M. and Law, M. I. Eye-specific termination bands in tecta of three-eyed frogs. Science 202, 639 (1978).

[20] Crair, M. Neuronal activity during development: permissive or instructive? Curr. Opin. Neurobiol. 9, 88 (1999).

[21] Crair, M., Gillespie, D., and Stryker, M. The role of visual experience in the development of columns in cat visual cortex. Science 279, 566 (1998).

[22] Creutzfeldt, O. Cortex Cerebri : Performance, Structural and Functional Organization of the Cortex. Oxford University Press, Oxford (UK) (1995).

[23] Cross, M. and Hohenberg, P. Pattern formation outside of equilibrium. Rev. Mod. Phys. 65, 851 (1993).

[24] Cross, M., Meiron, D., and Tu, Y. Chaotic domains: A numerical investigation. Chaos 4, 607 (1994).

[25] Crowley, J. and Katz, L. Ocular dominance development revisited. Curr Opin Neurobiol. 12, 104 (2002).

[26] Das, A. and Gilbert, C. D. Topography of contextual modulations mediated by short-range interactions in primary visual cortex. Nature 399, 655 (1999). 
[27] DeBruyn, E., Casagrande, V., Beck, P., and Bonds, A. Visual resolution and sensitivity of single cells in the primary visual cortex (v1) of a nocturnal primate (bush baby): correlations with cortical layers and cytochrome oxidase patterns. J. Neurophysiol. 69, 3 (1993).

[28] Decker, W., Pesch, W., and Weber, A. Spiral defect chaos in rayleigh-benard convection. Phys. Rev. Lett. 73, 648 (1994).

[29] Desai, N. S., Cudmore, R. H., Nelson, S. B., and G, T. G. Critical periods for experience-dependent synaptic scaling in visual cortex. Nat Neurosci. 5, 783 (2002).

[30] Durbin, R. and Mitchinson, G. A dimension reduction framework for understanding cortical maps. Nature 343, 644 (1990).

[31] Erwin, E. and Miller, K. Correlation-based development of ocularly matched orientation and ocular dominance maps: determination of required input activities. J. Neurosci. 18, 9870 (1998).

[32] Erwin, E., Obermayer, K., and Schulten, K. Models of orientation and ocular dominance columns in the visual cortex: A critical comparison. Neural Comp. 7, 425 (1995).

[33] Foeller, E. and Feldman, D. E. Synaptic basis for developmental plasticity in somatosensory cortex. Curr Opin Neurobiol. 14, 89 (2004).

[34] Gierer, A. and Müller, C. M. Development of layers, maps and modules. Current Opinion in Neurobiology 5, 91 (1995).

[35] Godde, B., Leonhardt, R., Cords, S. M., and Dinse, H. R. Plasticity of orientation preference maps in the visual cortex of adult cats. Proc. Natl. Acam. Sci. USA 99, 6352 (2002).

[36] Gödecke, I. and Bonhoeffer, T. Development of identical orientation maps for two eyes without common visual experience. Nature 379, 251 (1996).

[37] Hayashi, T. and Carthew, R. W. Surface mechanics mediate pattern formation in the developing retina. 2004 431, 647 (2004).

[38] Hensch, T. and Stryker, M. Columnar architecture sculpted by gaba circuits in developing cat visual cortex. Science 303, 1678 (2004).

[39] Hubel, D. H. and Wiesel, T. N. Receptive fields, binocular interaction and functional architecture in cat's visual cortex. J. Physiol. 160, 215 (1962). 
[40] Hubel, D. H. and Wiesel, T. N. Receptive fields, bionocular interaction and functional architecture in cat's visual cortex. J. Physiol. 160, 215 (1962).

[41] Hubener, M. Visual development: making maps in the dark. Curr. Biol. 8, 342 (1998).

[42] Humphrey, A., Albano, J., and Norton, T. Organization of ocular dominance in tree shrew striate cortex. Brain Res. 134, 225 (1977).

[43] Humphrey, A. and Norton, T. Topographic organization of the orientation column system in the striate cortex of the tree shrew (tupaia glis). J. Comp. Neurol. 192, 531 (1980).

[44] Kaschube, M., Wolf, F., Geisel, T., and Löwel, S. Genetic influence on quantitative features of neocortical architecture. J. Neurosci. 22, 7206 (2002).

[45] Kaschube, M., Wolf, F., Puhlmann, M., Rathjen, S., Schmidt, K. F., Geisel, T., and Löwel, S. The pattern of ocular dominance columns in cat primary visual cortex: intra- and interindividual variability of column spacing and its dependence on genetic background. Eur. J. Neurosci. 22, 7206 (2003).

[46] Katz, L. and Crowley, J. Development of cortical circuits: lessons from ocular dominance columns. Nat. Rev. Neurosci. 3, 34 (2002).

[47] Koch, A. and Meinhardt, H. Biological pattern formation: from basic mechanisms to complex structures. Rev. Mod. Phys. 66, 1481 (1994).

[48] Law, M., Zahs, K., and Stryker, M. Organization of primary visual cortex (area 17) in the ferret. J. Comp. Neurol. 278, 157 (1988).

[49] Lee, H. Y., Yahyanejad, M., and Kardar, M. Symmetry considerations and development of pinwheels in visual maps. Proc. Natl. Acam. Sci. USA 100, 16036 (2003).

[50] LeVay, S. and Nelson, S. B. Columnar organization of the visual cortex. In Vision and Visual Dysfunction, chapter 11, pages 266-315. Macmillan, Houndsmill (1991).

[51] Löwel, S. 2-deoxyglucose architecture of cat primary visual cortex. In Payne, B. and Peters, A. (editors), The Cat Primary Visual Cortex, chapter 2, pages 167-193. Academic Press, San Diego (2002).

[52] Löwel, S., Freeman, B., and Singer, B. Topographic organization of the orientation column system in large flat-mounts of the cat visual cortex: A 2deoxyglucose study. J. Comp. Neurol. 255, 401 (1987). 
[53] Lowel, S., Schmidt, K., Kim, D., Wolf, F., Hoffsummer, F., Singer, W., and Bonhoeffer, T. The layout of orientation and ocular dominance domains in area 17 of strabismic cats. Eur. J. Neurosci. 10, 2629 (1998).

[54] Löwel, S. and Singer, W. Tangential intracortical pathways and the development of iso-orientation bands in cat striate cortex. Dev. Brain Res. 56, 99 (1990).

[55] Löwel, S. and Singer, W. Selection of intrinsic horizontal connections in the visual cortex by correlated neuronal activity. Science 255, 209 (1992).

[56] Maldonado, P., Gödecke, I., Gray, C., and Bonhoeffer, T. Orientation selectivity in pinwheel centers in cat striate cortex. Science 276, 1551 (1997).

[57] Manneville, P. Dissipative Structures and Weak Turbulence. Academic Press, San Diego (1990).

[58] Melo, F., Umbanhowar, P., and Swinney, H. L. Transition to parametric wave patterns in a vertically oscillated granular layer. Phys. Rev. Lett. 72, 172 (1994).

[59] Mermin, N. D. Rev. Mod. Phys. 51, 591 (1979).

[60] Metcalf, T., Knight, J., and Jaeger, H. Surface patterns in shallow beds of vibrated granular material. Physica A, 236, 202 (1997).

[61] Miller, K. A model for the development of simple cell receptive fields and the ordered arrangement of orientation columns through activity-dependent competition between on- and off-center inputs. J. Neurosci. 14, 409 (1994).

[62] Miller, K. D., Erwin, E., and Kayser, A. Is the development of orientation selectivity instructed by activity? J. Neurobiol 41, 44 (1999).

[63] Mountcastle, V. Modality and topographic properties of single neurons of cat's somatic sensory cortex. J. Neurophysiol. 20, 408 (1957).

[64] Müller, T., Stetter, M., Hübener, M., Sengpiel, F., Bonhoeffer, T., Gödecke, I., Chapman, B., Löwel, S., and Obermayer, K. An analysis of orientation and ocular dominance patterns in the visual cortex of cats and ferrets. Neural Comput. 12, 2573 (2000).

[65] Nass, M. M. and Cooper, L. N. A theory for the development of feature detecting cells in visual cortex. Biol. Cybernetics 19, 1 (1975).

[66] Obermayer, K. and Blasdel, G. G. Singularities in primate orientation maps. Neural Comput. 9, 555 (1997). 
[67] Obermayer, K., Ritter, H., and Schulten, K. A principle for the formation of cortical feature maps. Proc. Natl. Acad. Sci. USA 87, 8345 (1990).

[68] Olavarria, J. Callosal connections correlate preferentially with ipsilateral cortical domains in cat areas 17 and 18, and with contralateral domains in the 17/18 transition zone. J. Comp. Neurol. 433, 441 (2001).

[69] Olavarria, J. Influence of topography and ocular dominance on the functional organization of callosal connections in cat striate cortex. In Payne, B. and Peters, A. (editors), The Cat Primary Visual Cortex, chapter 6, pages 259-318. Academic Press, San Diego (2002).

[70] Polimeni, J., Granquist-Fraser, D., Wood, R., and Schwartz, E. Physical limits to spatial resolution of optical recording: clarifying the spatial structure of cortical hypercolumns. Proc. Natl. Acad. Sci. USA 102, 4158 (2005).

[71] Press, W., Flannery, B., Teukolsky, S., and Vetterling, W. Numerical Recipes. Cambridge University Press, Cambridge, 2nd edition (1992).

[72] Rathjen, S., Engelmann, R., Struif, S., Kaulisch, T., Stiller, D., and Löwel, $\mathrm{S}$. The growth of cat cerebral cortex in postnatal life: a magnetic resonance imaging study. Eur. J. Neurosci. 18, 1797 (2003).

[73] Rosa, M. G., Casagrande, V. A., Preuss, T., and Kaas, J. H. Visual field representation in striate and prestriate cortices of a prosimian primate (galago garnetti). J. Neurophysiol. 77, 3193 (1997).

[74] Roxin, A. and Riecke, H. Rotating convection in an anisotropic system. Phys. Rev. E (2002).

[75] Ruthazer, E. and Stryker, M. The role of activity in the development of longrange horizontal connections in area 17 of the ferret. J. Neurosci. 16, 7253 (1996).

[76] Salazar, R. F., Konig, P., and Kayser, C. Directed interactions between visual areas and their role in processing image structure and expectancy. Eur J Neurosci. 20, 1391 (2004).

[77] Salin, P. A., Kennedy, H., and Bullier, J. Spatial reciprocity of connections between areas 17 and 18 in the cat. Can J Physiol Pharmacol. 73, 1339 (1995).

[78] Schmidt, K. E., Kim, D. S., Singer, W., Bonhoeffer, T., and Lowel, S. Functional specificity of long-range intrinsic and interhemispheric connections in the visual cortex of strabismic cats. J Neurosci. 17, 5480 (1997). 
[79] Schuett, S., Bonhoeffer, T., and Hubener, M. Pairing-induced changes of orientation maps in cat visual cortex. Neuron 32, 325 (2001).

[80] Schummers, J., Marino, J., and Sur, M. Synaptic integration by v1 neurons depends on location within the orientation map. Neuron 36, 969 (2002).

[81] Sengpiel, F., Gödecke, I., Stawinski, P., Hübener, M., Löwel, S., and Bonhoeffer, T. Intrinsic and environmental factors in the development of functional maps in cat visual cortex. Neuropharmacology 37, 607 (1998).

[82] Sengpiel, F., Stawinski, P., and Bonhoeffer, T. Influence of experience on orientation maps in cat visual cortex. Nat. Neurosci. 2, 727 (1999).

[83] Sharma, J., Angelucci, A., and Sur, M. Induction of visual orientation modules in auditory cortex. Nature 404, 841 (2000).

[84] Shinbrot, T. Competition between randomizing impacts and inelastic collisions in granular pattern formation. Nature 389, 32 (1997).

[85] Shmuel, A. and Grinvald, A. Coexistence of linear zones and pinwheels within orientation maps in cat visual cortex. PNAS 97, 5568 (2000).

[86] Shouval, H. Z., Goldberg, D. H., Jones, J. P., Beckerman, M., and Cooper, L. N. Structured long-range connections can provide a scaffold for orientation maps. J. Neurosci. 20, 1119 (2000).

[87] Sincich, L. and Blasdel, G. Oriented axon projections in primary visual cortex of the monkey. J. Neurosci. 21, 4416 (2001).

[88] Sur, M. and Leamey, C. Development and plasticity of cortical areas and networks. Nat. Rev. Neurosci. 2, 251 (2001).

[89] Swift, J. and Hohenberg, P. Hydrodynamic fluctuations at the convective instability. Phys. Rev. A 15, 319 (1977).

[90] Swindale, N. A model for the formation of orientation columns. Proc. R. Soc. Lond. 215, 211 (1982).

[91] Swindale, N. Iso-orientation domains and their relationship with cytochrome oxidase patches. In Rose, D. and Dobson, V. (editors), Models of the Visual Cortex, chapter 47, pages 452-461. Wiley, New York (1985).

[92] Swindale, N. The development of topology in the visual cortex: a review of models. Network: Computation in Neural Systems 7, 161 (1996).

[93] Swindale, N., Matsubara, J., and Cynader, M. Surface organization of orientation and direction selectivity in cat area 18. J. Neursci. 7, 1414 (1987). 
[94] Thomas, P. J. and Cowan, J. D. Symmetry induced coupling of cortical feature maps. Phys. Rev. Lett. 92, 188101 (2004).

[95] Treue, S. Climbing the cortical ladder from sensation to perception. Trends Cogn Sci. 7, 469 (2003).

[96] Turing, A. The chemical basis of morphogenesis. Philos. Trans. R. Soc. London 237, 37 (1952).

[97] Tusa, R., Palmer, L., and Rosenquist, A. The retinotopic organization of area 17 (striate cortex) in the cat. J. Comp. Neurol. 177, 213 (1978).

[98] Tusa, R., Rosenquist, A., and Palmer, L. Retinotopic organization of areas 18 and 19 in the cat. J. Comp. Neurol. 185, 657 (1979).

[99] Van Hooser, S. D., A, H. J., Chung, S., B, N. S., and J, T. L. Orientation selectivity without orientation maps in visual cortex of a highly visual mammal. J. Neurosci. 25, 19 (2005).

[100] von der Malsburg, C. Self-organization of orientation sensitive cells in the striate cortex. Kybernetik 14, 85 (1973).

[101] Weliky, M. and Katz, L. C. Functional mapping of horizontal connections in developing ferret visual cortex: Experiment and modeling. J. Neurosci. 14, 7291 (1994).

[102] White, L., Bosking, W., Williams, S., and Fitzpatrick, D. Maps of central visual space in ferret $\mathrm{v} 1$ and $\mathrm{v} 2$ lack matching inputs from the two eyes. J. Neurosci. 19, 7089 (1999).

[103] White, L., Coppola, D., and Fitzpatrick, D. The contribution of sensory experience to the maturation of orientation selectivity in ferret visual cortex. Nature 411, 1049 (2001).

[104] Wimbauer, S., Gerstner, W., and van Hemmen, J. L. Analysis of a correlationbased model for the development of orientation-selective receptive fields in the visual cortex. Network 9, 449 (1998).

[105] Wolf, F. Methods and models in neurophysics, Les Houches, Session LXXX, chapter 12. Elsevier, Amsterdam (2005).

[106] Wolf, F., Bauer, H.-U., Pawelzik, K., and Geisel, T. Organization of the visual cortex. Nature 382, 306 (1996).

[107] Wolf, F. and Geisel, T. Spontaneous pinwheel annihilation during visual development. Nature 395, 73 (1998). 
[108] Wolf, F. and Geisel, T. Universality in visual cortical pattern formation. J. Physiol. Paris 97, 253 (2003).

[109] Xu, X., Bosking, W., Sary, G., Stefansic, J., Shima, D., and Casagrande, V. Functional organization of visual cortex in the owl monkey. J. Neurosci. 24, 6237 (2004).

[110] Xu, X., Bosking, W., White, L., Fitpatrick, D., and Casagrande, V. Functional organization of visual cortex in the prosimian bush baby revealed by optical imaging of intrinsic signals. J. Neurophysiol. (2005).

[111] Xu, X., Collins, C., Kaskan, P., Khaytin, I., Kaas, J., and Casagrande, V. Optical imaging of visually evoked responses in prosimian primates reveals conserved features of the middle temporal visual area. Proc. Natl. Acad. Sci. USA 101, 2566 (2005). 


\section{Acknowledgments}

I shall thank the people who made this thesis possible.

I gladly thank Theo Geisel for giving me the opportunity to work in his group. 'The Institute' is a remarkable place with remarkable people in it. I also highly appreciate his efforts to bring the Bernstein Center for Computational Neuroscience to Göttingen.

I owe warm thanks to Fred Wolf for his support, for many inspiring discussions and his belief that this endeavor would finally work out. Also I thank him for teaching me to stand on my own feet, scientifically.

From Siegrid Löwel, IfN Magdeburg, I learned a lot about biology and about writing. I also thank her for providing experimental data (Chapter 3 and 8 ) and many, many valuable comments.

I thank Len E. White and David M. Coppola, Duke University, for providing experimental data (Chapter 4) and for annual encouraging discussions.

Karl-Friedrich Schmidt, IfN Magdeburg, and Hubert Dinse and Klaus Kreikemeier, University Bochum, also provided excellent data (Chapter 8) and other stimulating input. I thank Klaus for unforgettable days in Lisbon.

Denny Fliegner, Dirk Brockmann, York-Fabian Bensen and Ragnar Fleischmann helped when help seemed not so near.

I thank Corinna Trautsch, Katharina Jeremias and Regina Wunderlich for management and support.

In particular, I would like to thank Michael Schnabel with whom I shared a lot, but as I must regret, still not enough during the past years.

Also Michael Herrmann helped me enormously. He is a constant source of relativization.

Matthias Bethge has always been a great fellow, starting discussions in any condition and at any time.

I thank Florentin Wörgötter for constantly raising doubts about the significance of my research. For years now.

I appreciate Dominik Heide as a 'sophisticated trash talk buddy' for disport.

I would also like to thank Paulo Belli and Dirk Brockmann for music.

I thank Majda Attaoui Kadiri and Katharina Wesenick for applied chaos and mental support and my other friends for showing interest in my research.

I thank my parents for patience and open-mindedness. 
Finally, I want to thank Claudia Benze. Her part was essential. She provided the counterbalance to this work. 


\section{Lebenslauf}

\section{Persönliche Daten}

$\begin{array}{ll}\text { Name } & \text { Matthias Kaschube } \\ \text { Geburtsdatum } & 03.12 .1973 \\ \text { Geburtsort } & \text { Bergisch Gladbach } \\ \text { Staastangehörigkeit } & \text { Deutsch }\end{array}$

\section{Ausbildung}

1993

Abitur am Schiller-Gymnasium in Frankfurt am Main

$1993-1995$

Zivildienst

$1995-1997$

Studium der Physik (Diplom) und der Philosophie an der Johann Wolfgang Goethe-Universität, Frankfurt am Main

1996

Vordiplom in Physik an der Johann Wolfgang Goethe-Universität, Frankfurt am Main

1997 - $2000 \quad$ Fortsetzung des Studiums der Physik (Diplom) an der Georg-AugustUniversität, Göttingen

1998

Dreimonatiges Praktikum am Leibniz-Institut für Neurobiologie, Magdeburg, Arbeitsgruppe Prof. Dr. S. Löwel

Diplom in Physik an der Georg-August-Universität, Göttingen

seit 2000

Doktorand in der Abteilung Nichtlineare Dynamik (Prof. Dr T. Geisel) am Max-Planck-Institut für Dynamik und Selbstorganisation, Göttingen

Sommerschule „D ynamics of Neural Networks“(D . Kleinfeld, S. Seung, M. Tsodyks), Institute for Theoretical Physics UCSB, Santa Barbara, USA B. Gutkin, D. Hansel, Meunier, I. Segev), Ecole d'ete de Physique theoretique, Les Houches, Frankreich 